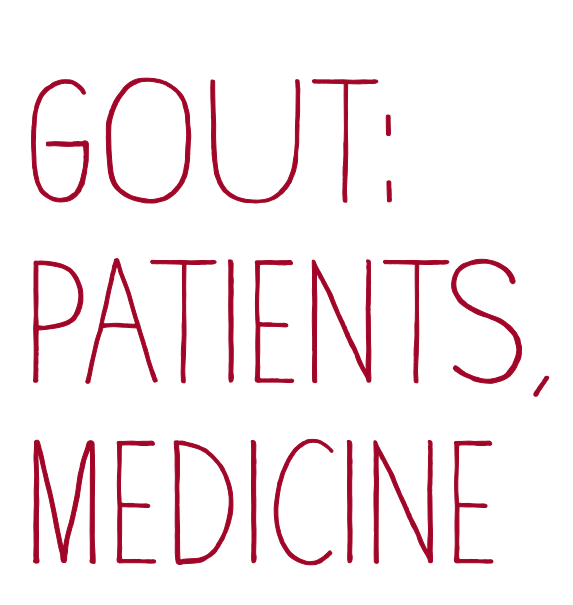

AND

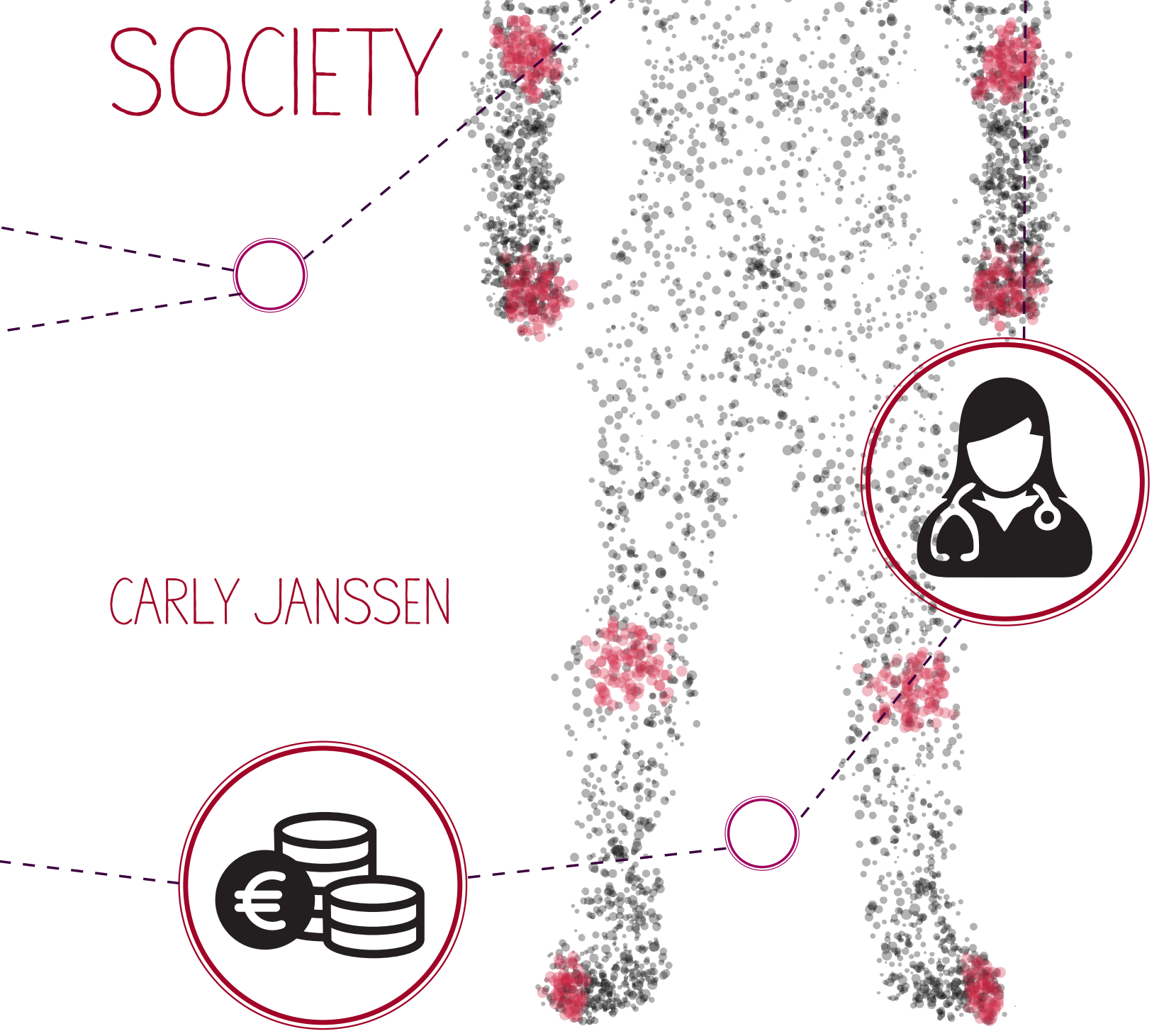

H. H.

2.

$\therefore 0^{3}$

20000001

$\therefore 8$ \% -

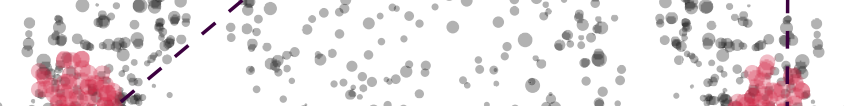





\section{GOUT: \\ PATIENTS, MEDICINE AND SOCIETY}

Carly Janssen 
The printing of this thesis was financially supported by Swedish Orphan Biovitrum AB.

Cover design: Ilse Modder, www.ilsemodder.nl

Printed by: Gildeprint, The Netherlands, www.gildeprint.nl

Lay-out: Ilse Modder, www.ilsemodder.nl

ISBN: $\quad 978-90-365-4811-3$

DOI: $\quad 10.3990 / 1.9789036548113$

(C) 2019 Carly A. Janssen, Enschede, The Netherlands.

All rights reserved. No parts of this thesis may be reproduced, stored in a retrieval system or transmitted in any form or by any means without permission of the author. 


\section{GOUT: \\ PATIENTS, MEDICINE AND SOCIETY}

PROEFSCHRIFT

ter verkrijging van

de graad van doctor aan de Universiteit Twente,

op gezag van de rector magnificus,

prof. dr. T.T.M. Palstra,

volgens besluit van het College voor Promoties

in het openbaar te verdedigen

op donderdag 11 juli 2019 om 10.45 uur

door

Carly Ann Janssen

geboren op 27 december 1988

te Nijmegen, Nederland 
Dit proefschrift is goedgekeurd door de promotor prof. dr. M.A.F.J. van de Laar en de copromotoren dr. M.A.H. Oude Voshaar en dr. H.E. Vonkeman. 
Samenstelling promotiecommissie

\section{Voorzitter/secretaris}

Prof. dr. T.A.J. Toonen (Universiteit Twente)

\section{Promotor}

Prof. dr. M.A.F.J. van de Laar (Universiteit Twente)

\section{Copromotoren}

Dr. M.A.H. Oude Voshaar (Universiteit Twente)

Dr. H.E. Vonkeman (Universiteit Twente)

\section{Leden}

Prof. dr. R. Westhovens (Universitair Ziekenhuis Leuven, Leuven, België)

Prof. dr. A. So (Universitair Medisch Centrum Vaud, Lausanne, Zwitserland)

Prof. dr. T. Bardin (Lariboisière Ziekenhuis, Parijs, Frankrijk)

Prof. dr. E.T. Bohlmeijer (Universiteit Twente)

Dr. E. Taal (Universiteit Twente) 

"I have a name men dread and loathe to hear; They call me Gout, a fearsome scourge to men; I bind their feet in sinew-knotting cords, When I have swept unseen into their joints."

- Lucian of Samosata (125 AD - 180 AD, from 'Swift-of-Foot') - 



\section{CONTENTS}

Chapter $1 \quad$ General introduction \& Outline of thesis

Part I Clinical management of gout and hyperuricemia

Chapter 2 Anakinra for the treatment of acute gout flares: a randomized,

double-blind, placebo-controlled, active-comparator, noninferiority trial

Chapter 3 Prognostic factors associated with early gout flare recurrence in patients initiating urate-lowering therapy during an acute gout flare

Chapter 4 Quality of care in gout: a clinical audit on treating to the target with urate lowering therapy in real-world gout patients

Chapter 5 A retrospective analysis of medication prescription records for determining the levels of compliance and persistence to uratelowering therapy for the treatment of gout and hyperuricemia in The Netherlands

Part II

Chapter 6

Chapter 7

Part III

Chapter 8

Chapter 9

Chapter 10

\section{Patient-reported outcome measures in gout}

A systematic literature review of patient-reported outcome measures used in gout: an evaluation of their content and measurement properties

Development and validation of a patient-reported gout attack intensity score for use in gout clinical studies

\section{Societal burden of gout}

Model-based cost-effectiveness analyses comparing combinations of urate lowering therapy and anti-inflammatory treatment in newly diagnosed gout patients

Summary \& General Discussion

Appendices

Dutch summary I Nederlandse samenvatting

Acknowledgements (in Dutch) I Dankwoord

About the author

List of publications 181 188

92 196 
<smiles>CO[C@H]1O[C@H](O)[C@H](O)O1</smiles> 
General introduction \&

Outline of thesis 


\section{GENERAL INTRODUCTION}

\section{History of gout}

The first text descriptions of gouty arthritis were made by Egyptians around $2640 \mathrm{BC}$ [1]. Centuries later, around $400 \mathrm{BC}$. Hippocrates is believed to have made the first accurate clinical description of an acute gout attack in the first metatarsophalangeal joint, which was at that time referred to as podagra, literally meaning 'foot-grabber' ${ }^{[2]}$. In his work, he also clearly describes a relationship between gout and lifestyle, forming the basis for the long-lasting classification of podagra as the "arthritis of the rich" or a "disease of kings"; A group living abundant lives and frequent sufferers of gout. Six centuries later, Galen (130-200 AD), was the first to describe tophi (lumps of monosodium urate (MSU) crystals) as chalk-like structures ${ }^{[3]}$. The descriptions of Hippocrates and Galen endured throughout medical history, yet only around 1200 AD was the term gout introduced for the first time ${ }^{[4]}$.

In the $16^{\text {th }}$ century, developments in the understanding of gout became more precise and more in line with the disease as we know it today. In 1683, the English physician Sydenham distinguished gout from other forms of arthritis, and differentiated an acute and advanced (chronic) form ${ }^{[5]}$. Around the same time, in 1679, Antonie van Leeuwenhoek, was the first to describe the appearance of the contents of a tophus as seen through his microscope, although the chemical composition of uric acid crystals was not yet known at the time ${ }^{[6]}$. Approximately one hundred years later in 1776, the Swedish chemist Scheele isolated the chemical component uric acid, and Woolaston, an English chemist, retrieved urate from a tophus in his own ear ${ }^{[4]}$. However, only in 1961 did McCarty and Hollander publish experiments which demonstrated that synovial fluid contains MSU crystals, which would serve as a basis for the diagnosis of gout ${ }^{[7]}$. From this point onwards, the understanding of gout grew rapidly, with developments in the areas of treatment as well as the understanding of its pathophysiology ${ }^{[8]}$.

\section{Pathophysiology and clinical manifestations}

Gout is a MSU crystal-induced inflammatory disease of the joint, belonging to the family of rheumatic diseases. Prolonged hyperuricemia may result in the formation of MSU crystals when serum urate (SUA) levels surpass the saturation point of MSU of $>6.8 \mathrm{mg} /$ $\mathrm{dL}$, followed by their deposition in and between soft tissue and joints, in particular the first metatarsophalangeal joint ${ }^{[9,10]}$. Presence of MSU crystals in the synovial fluid of joints may attract macrophages and monocytes that attempt to phagocytize the crystals, leading to intracellular activation of the NALP3 inflammasome that results in excretion of the cytokine interleukin (IL)-1 ${ }^{[11]}$. By binding to receptors on local endothelial cells 
and macrophages, excreted IL-1 $1 \beta$ signals these cells to produce more and other proinflammatory proteins, thus amplifying the inflammatory response, generally leading to localized symptoms as joint pain, swelling, erythema and tenderness of the affected joint ${ }^{[12]}$. The (sudden) onset of such an event is commonly referred to as an acute gout flare or attack, and may also be associated with general symptoms as fever and malaise, or physical impairment and decreased quality of life ${ }^{[13]}$.

Untreated gout and hyperuricemia may lead to worsening of the disease, reflected by accumulation of MSU crystals within joints and soft tissues, that could lead to the development of tophi, and repeated episodes of acute gout flares. At this stage, joint damage and deformation may occur. Although hyperuricemia is the predominant risk factor for developing gout, the presence of comorbidities (e.g., renal disease, hypertension, obesity) and genetic factors, are further underlying factors that contribute to increased urate levels and therefore the development of gout ${ }^{[14]}$. Also modifiable lifestyle factors as diet, including high purine turn-over, and exercise have been associated with elevated urate levels and thus gout development ${ }^{[15]}$. In general, patients with gout have an increased risk for cardiovascular diseases, mainly related to the hyperuricemia ${ }^{[14]}$.

\section{Epidemiology}

Gout is one of the most prevalent forms of inflammatory arthritis ${ }^{[16]}$. The prevalence of gout appears to increase over time, and varies greatly across geographic regions, with countries as Mexico and Cuba having prevalence estimates ranging from $0.3-$ $0.4 \%$, whereas in the US and UK, $3.9 \%$ and $3.2 \%$ of adults have been reported to have gout, respectively ${ }^{[17-20]}$. Prevalence estimates even $>6 \%$ have been reported in specific subpopulations, such as in the male Maori population within New Zeeland ${ }^{[21]}$. In general, the prevalence of gout is higher in wealthier, developed countries, compared to developing countries, which may be attributed to the increasing longevity of these populations and increasing (life-style associated) comorbid conditions ${ }^{[16]}$.

\section{Clinical management of gout and hyperuricemia}

For the management of gout in clinical practice, a distinction can be made between the treatment of acute gout flares by alleviating pain and inflammation on the one hand, and the lowering and monitoring of SUA levels for preventing future attacks and improving overall prognosis, on the other. Various effective anti-inflammatory and antihyperuricemia treatment options are currently available to treat gout and hyperuricemia, respectively. As such, from a clinical point of view, gout is often considered to be a well treatable and manageable disease. Still, reports are increasingly highlighting concerns 
on the management of gout, with many patients achieving unfavorable outcomes in both the acute phase of the disease, but also in the long-term ${ }^{[19,22,23]}$.

\section{Acute gout flares}

For the treatment of acute gout flares colchicine, non-steroidal anti-inflammatory drugs (NSAIDs), and corticosteroids are the three first-line treatment agents recommended by international management guidelines, as their efficacy for the treatment of acute gout flares has been well documented in the past decades ${ }^{[24-26]}$. In general, these agents allow for a rapid decrease in pain and inflammation of the affected joint and make the acute phase of the disease well treatable. However, treatment success might be limited by adverse drug reactions. Moreover, as presence of multimorbidity is common in patients with gout, for example comorbidities like cardiovascular disease, renal disorders or metabolic disorders, treatment with these conventional therapies may be difficult ${ }^{[27]}$. Such comorbidities may cause contraindications to the standard treatment options, subsequently leaving gout patients untreated for their flares as treatment with the conventional therapies is thus hampered or deemed unsuited. For these patients, and in patients with frequent occurring flares, treatment with an IL-1 inhibitor may be considered as an alternative ${ }^{[25]}$. Yet, to date, only the IL-1 $\beta$ monoclonal antibody canakinumab has been registered for treatment of acute gout flares in Europe. Unfortunately, canakinumab is only available for gout patients with at least three gout attacks per year, in whom treatment with NSAIDs, colchicine or corticosteroids are contraindicated, ineffective or intolerable. In addition, applicability of canakinumab is limited by its high price (i.e. €12,000 per injection in the Netherlands), which might discourage its use in clinical practice. Moreover, rilonacept, another IL-1 inhibitor, was shown to not provide additional or superior pain-relief when used as an add-on therapy to standard of care, compared to standard of care NSAID in treating gout flares ${ }^{[28]}$. As such, treatment alternatives for those gout patients who do not meet the indicative requirements for canakinumab, but are also difficult to treat using conventional therapies, remain limited.

\section{Hyperuricemia}

Treating hyperuricemia with urate lowering therapy (ULT) to prevent the progression of the disease and the reoccurrence of gout flares is a major part of the long-term management of gout. The ultimate goal of ULT is to lower SUA to a level that induces dissolution of MSU crystal depositions, and to maintain SUA levels below the saturation threshold for MSU crystal formation. In general, with every patient with crystal-proven gout, ULT should be considered and discussed, according to gout guidelines ${ }^{[25,29]}$. In patients with frequent flares, tophi, renal stones and urate arthropathy, adequate ULT 
is indicated ${ }^{[25,29]}$. Unfortunately, despite these recommendations and the availability of effective ULT, gout flare recurrences are still common in patients on ULT ${ }^{[30]}$. Therefore, research focusing on improving the clinical outcomes of gout patients has gained priority in this field, including the prevention of gout flares and determining best practices ${ }^{[31]}$.

In contemporary gout management best practices, a treat to target (T2T) approach is advised in lowering SUA levels with ULT in gout patients. T2T is a disease management strategy in which well-defined, clinically relevant endpoints are aggressively pursued by means of frequent monitoring of the relevant endpoints, and making therapeutic adjustments aimed at achieving the treatment target, and to maintain this over time ${ }^{[32]}$. A T2T approach was first applied in the field of cardiovascular diseases, for example, in the management of hypertension and hypercholesterolemia ${ }^{[33]}$. Later on, the concept of T2T was also introduced in other medical disciplines, including the management of metabolic diseases (i.e. diabetes), and rheumatic conditions. In rheumatology, a T2T concept has first been adopted in the treatment of patients with rheumatoid arthritis, where the target for clinical remission of disease activity is the goal ${ }^{[34-36]}$. In other rheumatic conditions, including gout and hyperuricemia, treating to a target is still a fairly new concept.

In gout patients, evidence is increasingly showing that SUA levels below $6 \mathrm{mg} / \mathrm{dL}$ (360 $\mu \mathrm{mol} / \mathrm{L}$ ) is the minimal target at which benefits from ULT may be achieved in all gout patients, supported by studies wherein this target has been associated with decreased flare frequencies and decreased tophi size in gout patients ${ }^{[30,37]}$. A more stringent target of $5 \mathrm{mg} / \mathrm{dL}(300 \mu \mathrm{mol} / \mathrm{L}$ ) has also been proposed for patients with severe (tophaceous) gout, although less evidence is available to support this target ${ }^{[25]}$. Consequently, international gout management guidelines (i.e. ACR, EULAR) have endorsed these SUA levels as target levels when initiating patients on ULT ${ }^{[25,29]}$. In meeting these SUA target levels, a T2T approach is recommended. This implies that SUA levels are monitored over time, and that adjustments in ULT treatment dosages or ULT agents are made at the discretion of the treating caregiver in order to reach these predefined SUA target levels. Though adopted in these guidelines, evidence supporting the added value of a T2T strategy in lowering SUA levels in gout patients remains scarce ${ }^{[38]}$.

While the concept of T2T in (effectively) lowering and maintaining SUA levels with ULT is still relatively new and, as yet supported by few studies in gout, the overarching issue of ULT medication adherence in gout patients is well known and supported by various international studies ${ }^{[39]}$. Medication adherence plays a significant role in the degree of treatment success and disease prognosis ${ }^{[40]}$. While varying definitions of medication 
adherence persist in literature, the International Society for Pharmacoeconomics and Outcomes Research (ISPOR), Medication and Compliance Work Group defines medication adherence (also called 'compliance') as "the extent to which a patient acts in accordance with the prescribed interval and dose of a dosing regimen" [41]. Persistence refers to "the duration of time from initiation to discontinuation of therapy".

In gout and hyperuricemia, poor ULT medication adherence and persistence may contribute to reoccurrence of flares or worsening of disease, since SUA levels are insufficiently lowered ${ }^{[42]}$. Therefore, for achieving favorable disease and patient outcomes, it is of utmost importance that patients are adherent to their ULT. Although various international studies have reported on poor ULT medication adherence in gout patients, whether this issue also exists among gout patients in the Netherlands remains unknown.

\section{Measuring outcomes that matter to patients with gout}

The biomedical perspective is heavily emphasized in the management of gout, with the resolution of inflammation and normalization of SUA levels as central objectives. However, it is increasingly recognized that disease specific biomedical outcomes by themselves fail to capture aspects of health status that are of importance to patients, as well as other stakeholders. In fact, it is the impact of biomedical factors (i.e. signs and symptoms) on patient's social role functioning or their ability to perform daily activities what usually prompts them to seek medical attention, rather than the biological factors themselves ${ }^{[43]}$. Also in gout, patients report relevant outcome domains impacted by disease to be related to physical functioning, performance of daily activities, diet, work and productivity, sleep, social functioning and emotional wellbeing ${ }^{[44-46]}$. For assessing the consequences and impact of disease in dimensions such as these from a patient's perspective, patient reported outcome measures (PROMs) are useful.

Originally, PROMs were introduced in the field of health services research, to meet an increasing societal demand (from payers, caregivers, governmental bodies) for outcomes data in the second half of the $20^{\text {th }}$ century. A variety of generic and diseasespecific PROMs were developed during that time to help various stakeholders make informed and sound resource allocation decisions ${ }^{[47,48]}$. These measures subsequently also became popular in clinical trials, and were increasingly used to support labeling claims for newly developed medical products ${ }^{[49]}$.

The Health Assessment Questionnaire (HAQ) disability index, and the Arthritis Impact Measurement Scales (AIMS) were among the first PROMs to be specifically developed in 
the field of rheumatology ${ }^{[50,51]}$. Over time, the importance of measuring patient outcomes with use of PROMs grew in this field, which was in part driven by the recognition that PROMs better predict outcomes such as early mortality, work disability and joint replacement, compared to biological (clinical) components in patients with rheumatoid arthritis ${ }^{[43,52,53]}$. In addition, patient outcomes were shown to correlate with clinical measures in patients with rheumatoid arthritis ${ }^{[43]}$, and PROMs were frequently shown to be more sensitive to change than clinical outcomes ${ }^{[54]}$. Nowadays, various patient reported core outcome domains, for different rheumatic conditions, have been defined by the Outcomes Measures in Rheumatology Clinical Trials (OMERACT) initiative for clinical trials ${ }^{[55,56]}$ and the International Consortium for Health Outcome Measurement (ICHOM) for measuring quality of care in daily practice ${ }^{[57]}$.

Also in gout, PROMs have made a significant contribution to understanding the impact gout may have on patients from a patient's perspective ${ }^{[58]}$. This includes outcomes related to patient's symptom experience, work disability, but also the degree of physical disability, and emotional and social wellbeing, overall determining someone's overall quality of life ${ }^{[59,60]}$. Following developments in other rheumatic conditions, patient outcomes but also clinical outcomes, that are considered relevant for in gout clinical trials have been defined by the OMERACT working group gout ${ }^{[61]}$. In addition, for each of the patient outcomes PROMs have been identified that pass the OMERACT filter of truth (valid), discrimination (reliable), and feasibility. Despite their recommendation for use in clinical studies, evidence supporting the validity and reliability of PROMS is currently limited, as well as the availability of gout-specific PROMs.

\section{Societal burden of gout}

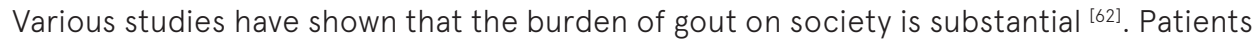
with gout suffer from loss of work productivity and high costs of illness, which have been shown to increase with the severity of disease ${ }^{[62-64]}$. The burden is likely to increase further in the near future, in light of the increasing prevalence of gout, the chronic nature of hyperuricemia and the development of new pharmacological, and potentially expensive treatment options. Considering these developments, gaining insight into the costs and benefits associated with (new) gout treatment interventions will therefore become increasingly important. In particular as the societal health care expenditures are already limited, and resources are in need of effective allocation ${ }^{[65]}$. For this purpose, health economic evaluations have become a valuable tool in various research domains. However, for gout limited health economic evaluations comparing costs and effectiveness of treatment options have been reported on. 


\section{OUTLINE OF THESIS}

\section{Part I: Clinical management of gout and hyperuricemia}

\section{Anakinra for treating acute gout flares}

Alternative treatment options for patients with acute gout flares are currently needed. Gout patients commonly suffer from comorbidities that hamper the applicability of conventional first-line treatment options, including colchicine, NSAIDs and corticosteroids, due to contraindications ${ }^{[27.66]}$. In addition, occurrence of adverse treatment reactions or inefficacies, may make treatment with these therapies difficult ${ }^{[67]}$. Alternatively, for those patients who cannot be treated with conventional therapies, treatment with an IL-1 inhibitor may be considered as an alternative ${ }^{[25]}$. Currently, the $\mathrm{IL}-1 \beta$ monoclonal antibody canakinumab is the only registered IL-1 inhibitor for gout in Europe, indicated for patients with frequent occurring flares ( $\geq 3$ flares per year), in whom the conventional treatment are contraindicated, intolerable of ineffective. However, this narrow indication, as well as its high price, may hamper its applicability in daily clinical practice. As such, treatment alternatives for treating patients with acute gout flares remain limited.

Anakinra is an alternative $\mathrm{IL}-1$ receptor antagonist, which competitively blocks the binding of excreted $\mathrm{IL}-1$ (both $\mathrm{IL}-1 \beta$ and $\mathrm{IL}-1 \mathrm{a}$ ), consequently suppressing the biochemical inflammatory response associated with gout. Various uncontrolled observational studies and one open-label study have reported on gout patients that have been treated with anakinra. These studies suggest that anakinra may work effectively for treating acute gout flares ${ }^{[68,69,78-83,70-77]}$. However, thus far no randomized controlled trials studying the efficacy and safety of anakinra in gout patients has been reported. Such trials are necessary for understanding whether anakinra is truly an effective and safe alternative for the treatment of acute gout flares. In particular in gout, randomized controlled trials for studying the efficacy of treatment options are desired considering the self-limiting course of disease. That is, the interpretation of results of uncontrolled, observational studies are hampered as the observed clinical improvement seen in these studies might also be a reflection of the self-limiting natural course of acute gout. Therefore, in Chapter 2 of this thesis, the results of a randomized controlled trial in which anakinra was compared to standard of care for the treatment of acute gout flares are described.

\section{Management of hyperuricemia}

Initiating ULT for lowering and managing SUA levels is believed to contribute to improving 
clinical outcomes in gout patients, including fewer gout flare recurrences ${ }^{[30]}$. It has therefore been recommended by gout management guidelines that ULT should be discussed and considered in all patients upon a definite diagnosis of gout ${ }^{[25,29]}$. In addition, early initiation of ULT is preferred, which may involve ULT being initiated upon the presentation of an acute gout flare ${ }^{[25,29,84]}$. As a protective measure, prophylaxis with colchicine or NSAID is also recommend when starting ULT to suppress the eliciting of a new flare ${ }^{[25]}$. Despite these recommendations, patients on ULT still commonly suffer from gout flare recurrences, particularly in the period directly following ULT initiation

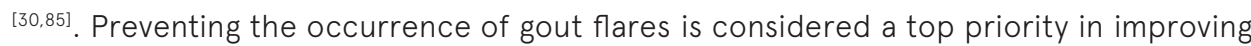
gout outcomes ${ }^{[31]}$. Therefore, clinical outcomes may improve when an understanding is gained regarding which factors may be associated with gout flare occurrences after initiation of ULT. An exploration of such variables may reveal areas of interest for future research, which could eventually help in determining whether a patient is at increased risk for developing gout flares when initiating ULT. However, to date not much is known about prognostic factors related to early ( $\leq 3$ months) gout flare recurrence, let alone in patients initiation ULT during an acute gout flare ${ }^{[85-89]}$. Therefore, the study in Chapter 3 aimed to determine which of various patient, clinical or patient-reported characteristics were prognostic of gout flare occurrence within the first three months after starting ULT during an acute gout episode.

For the management of gout and hyperuricemia, the principle of treating to a target in SUA levels with ULT has been adopted in various international management guidelines. However, current evidence supporting a T2T approach in gout is still rather weak ${ }^{[38]}$, and the 2016 published T2T recommendations for gout are solely based on consensus and expert opinion ${ }^{[90]}$. In order to support and understand the added value of a T2T strategy in the treatment of hyperuricemia, the study in Chapter 4 reflects on its implementation in clinical practice and reveals the clinical outcomes achieved in real-world gout patients.

A major issue in the long-term management of gout is poor ULT medication adherence by patients, potentially influencing outcomes seen in gout patients such as gout flare recurrences. This issue has been reported on by various international studies that have evaluated the levels of ULT compliance and persistence in their country. However, thus far, no such report has been attained for the Netherlands. Yet, in order to improve medication adherence in gout patients at a national level, insight into the status of prescribing patterns of physicians, as well as the levels of patient compliance and persistence is desired. This could consequently reveal whether and where potential areas of improvement in medication adherence lie for gout patients in the Netherlands. 
Therefore, the study in Chapter $\mathbf{5}$ determined the ULT dispensing patterns, as well as the levels of compliance and persistence of ULT among patients with gout and hyperuricemia in the Netherlands.

\section{Part II: PROMs in gout}

Improving outcomes assessment from the patient's perspective

Various gout outcome domains and associated PROMs have been endorsed based on the OMERACT filter of truth, discrimination and feasibility, for use in gout clinical trials ${ }^{[58,91]}$. However, equally important when using PROMs is that their use should be substantiated with evidence supporting their psychometric properties in the population of interest, to ensure that reliable and valid measurement of outcomes are made ${ }^{[43,92]}$. In addition, the methodological quality of the study should be considered. For this purpose, Consensusbased Standards for the selection of health Measurement Instruments (COSMIN), has created a checklist to evaluate the methodological quality of a study evaluating the measurement properties of an instrument ${ }^{[93]}$. In spite of their widespread use in gout clinical research, evidence on the psychometric properties of PROMs remains scare, in particular with regard to the methodological quality of the study applied. Therefore, in Chapter 6, we systematically reviewed which PROMs are currently available for gout clinical studies, and determined the level of evidence supporting their psychometric properties, while considering the methodological quality of the study. Gaining insight into the psychometric properties of the PROMs used in gout is essential, considering their widespread use for gout research purposes.

In acute gout clinical studies, flare diaries are often used to capture the severity of flarerelated symptoms from a patient's perspective, including levels of pain, tenderness and swelling of the joint affected by gout. Though this data is elaborately gathered in acute gout clinical studies, only pain is commonly used as a primary endpoint for evaluating efficacy for treatment. However, combining information from all three symptoms (i.e. pain, swelling, and tenderness), may provide a more comprehensive summary on gout flare symptom intensity, which may potentially be more responsive to change compared to the single-item components. In Chapter 7, we introduce and evaluate a gout-specific, patient-reported, gout attack intensity score (GAIS) for use in gout clinical studies, composed of the single-items pain, tenderness and swelling. 


\section{Part III: Societal burden of gout}

Health economic evaluation for gout

For gout, various cost-effectiveness studies have been done, comparing either treatment options available for acute gout flares (i.e. anti-inflammatory agents) or for hyperuricemia (i.e. ULT) ${ }^{[94-102]}$. However, a health economic model, wherein commonly used treatment strategies for acute gout flares and ULT may be compared simultaneously has not been developed for gout. Yet, such a model would allow for comparison of the costs and benefits of available treatment strategies in gout and hyperuricemia, and evaluate the added value of implementing new, more expensive drugs (i.e. anakinra) in the treatment arsenal of treating physicians. Therefore, in Chapter $\mathbf{8}$, a health economic model was developed to determine and compare the costs and benefits of treatment strategies, including treatments available for both the acute phase of the disease and the long-term management of hyperuricemia. 


\section{REFERENCES}

1. Schwartz SA. Disease of Distinction. EXPLORE. 2006;2(6):515-9.

2. Hippocrates. The Genuine Works of Hippocrates, vol I and II. New York: Wood and company. Edited and translated by Adams F; 1886.

3. Galen C. Claudii Galeni Opera Omni. Leipzig: CG Kühn;

4. Nuki G, Simkin PA. A concise history of gout and hyperuricemia and their treatment. Arthritis Res Ther. 2006;8(Suppl 1):S1.

5. MacKenzie CR. Gout and Hyperuricemia: an Historical Perspective. Curr Treat Options Rheumatol. 2015;1(2):119-30.

6. Mccarty DJ. A historical note: leeuwenhoek's description of crystals from a gouty tophus. Arthritis Rheum. $1970 ; 13(4): 414-8$

7. McCarty D, Hollander J. Identification of urate crystals in gouty synovial fluid. Ann Intern Med. 1961;54:45260.

8. Scott J. Gout: the last 50 years. J R Soc Med. 1996;89(11):634-7.

9. Martillo MA, Nazzal L, Crittenden DB. The crystallization of monosodium urate. Curr Rheumatol Rep. $2014 ; 16(2): 400$

10. Roddy E. Revisiting the pathogenesis of podagra: why does gout target the foot? J Foot Ankle Res. 2011;4(1):13.

11. Martinon F, Pétrilli V, Mayor A, Tardivel A, Tschopp J. Gout-associated uric acid crystals activate the NALP3 inflammasome. Nature. 2006;440(7081):237-41.

12. Pope RM, Tschopp J. The role of interleukin-1 and the inflammasome in gout: Implications for therapy. Arthritis Rheum. 2007:56(10):3183-8.

13. Becker MA, Schumacher HR, Benjamin KL, Gorevic P, Greenwald M, Fessel J, et al. Quality of life and disability in patients with treatment-failure gout. J Rheumatol. 2009;36(5):1041-8.

14. Richette P, Bardin T. Gout. Lancet. 2010;375:318-28.

15. Saag KG, Choi H. Epidemiology, risk factors, and lifestyle modifications for gout. Arthritis Res Ther. 2006;8 Suppl 1:S2.

16. Kuo C-F, Grainge MJ, Zhang W, Doherty M. Global epidemiology of gout: prevalence, incidence and risk factors. Nat Rev Rheumatol. 2015;11(11):649-62.

17. Peláez-Ballestas I, Sanin LH, Moreno-Montoya J, Alvarez-Nemegyei J, Burgos-Vargas R, Garza-Elizondo M, et al. Epidemiology of the rheumatic diseases in Mexico. A study of 5 regions based on the COPCORD methodology. J Rheumatol. 2011;Suppl. 86:3-8.

18. Reyes-Llerena GA, Guibert-Toledano M, Penedo-Coello A, Pérez-Rodríguez A, Baez-Dueñas RM, Charnicharo-Vidal R, et al. Community-based study to estimate prevalence and burden of illness of rheumatic diseases in Cuba: a COPCORD study. J Clin Rheumatol. 2009;15(2):51-5

19. Kuo C-F, Grainge MJ, Mallen C, Zhang W, Doherty M. Rising burden of gout in the UK but continuing suboptimal management: a nationwide population study. Ann Rheum Dis. 2015;74(4):661-7. 
20. Roddy E, Doherty M. Epidemiology of gout. Arthritis Res Ther. 2010;12(6):223.

21. Klemp P, Stansfield SA, Castle B, Robertson MC. Gout is on the increase in New Zealand. Ann Rheum Dis. $1997 ; 56: 22-6$

22. Doherty M, Jansen TL, Nuki G, Pascual E, Perez-Ruiz F, Punzi L, et al. Gout: why is this curable disease so seldom cured? Ann Rheum Dis. 2012;71(11):1765-70.

23. Edwards NL. Quality of care in patients with gout: Why is management suboptimal and what can be done about it? Curr Rheumatol Rep. 2011;13(2):154-9.

24. Wechalekar MD, Vinik O, Moi JHY, Sivera F, Van Echteld IAAM, van Durme C, et al. The efficacy and safety of treatments for acute gout: Results from a series of systematic literature reviews including cochrane reviews on intraarticular glucocorticoids, colchicine, nonsteroidal antiinflammatory drugs, and interleukin-1 inhibitors. J Rheumatol. 2014;92:15-25.

25. Richette P, Doherty M, Pascual E, Barskova V, Becce F, Castañeda-Sanabria J, et al. 2016 updated EULAR evidence-based recommendations for the management of gout. Ann Rheum Dis. 2017;76(1):29-42.

26. Khanna D, Khanna PP, Fitzgerald JD, Singh MK, Bae S, Neogi T, et al. 2012 American college of rheumatology guidelines for management of gout. part 2: Therapy and antiinflammatory prophylaxis of acute gouty arthritis. Arthritis Care Res. 2012;64(10):1447-61.

27. Bardin T, Richette P. Impact of comorbidities on gout and hyperuricaemia: An update on prevalence and treatment options. BMC Med. 2017;15(123):1-10.

28. Terkeltaub RA, Schumacher HR, Carter JD, Baraf HSB, Evans RR, Wang J, et al. Rilonacept in the treatment of acute gouty arthritis: a randomized, controlled clinical trial using indomethacin as the active comparator. Arthritis Res Ther. 2013;15:R25.

29. Khanna D, Fitzgerald JD, Khanna PP, Bae S, Singh MK, Neogi T, et al. 2012 American college of rheumatology guidelines for management of gout. part 1: Systematic nonpharmacologic and pharmacologic therapeutic approaches to hyperuricemia. Arthritis Care Res. 2012;64(10):1431-46.

30. Sarawate CA, Patel PA, Schumacher HR, Yang W, Brewer KK, Bakst AW. Serum Urate Levels and Gout Flares Analysis from managed care data. J Clin Rheumatol. 2006;12(2):61-5.

31. Dalbeth N, Choi HK, Terkeltaub R. Gout: A roadmap to approaches for improving global outcomes. Arthritis Rheumatol. 2017;69(1):22-34.

32. Smolen JS. Treat-to-target: Rationale and strategies. Clin Exp Rheumatol. 2012;30(Suppl.73):S2-6.

33. Atar D, Birkeland KI, Uhlig T. "Treat to target": Moving targets from hypertension, hyperlipidaemia and diabetes to rheumatoid arthritis. Ann Rheum Dis. 2010;69(4):629-30.

34. Smolen JS, Breedveld FC, Burmester GR, Bykerk V, Dougados M, Emery P, et al. Treating rheumatoid arthritis to target: 2014 update of the recommendations of an international task force. Ann Rheum Dis. $2016 ; 75(1): 3-15$

35. Schipper LG, Vermeer M, Kuper HH, Hoekstra MO, Haagsma CJ, Den Broeder AA, et al. A tight control treatment strategy aiming for remission in early rheumatoid arthritis is more effective than usual care treatment in daily clinical practice: A study of two cohorts in the Dutch Rheumatoid Arthritis Monitoring registry. Ann Rheum Dis. 2012;71(6):845-50. 
36. Vermeer M, Kuper HH, Hoekstra M, Haagsma CJ, Posthumus MD, Brus HLM, et al. Implementation of a Treatto-Target Strategy in very early Rheumatoid Arthritis. Arthritis Rheum. 2011;63(10):2865-72.

37. Perez-Ruiz F, Calabozo M, Pijoan JI, Herrero-Beites AM, Ruibal A. Effect of urate-lowering therapy on the velocity of size reduction of tophi in chronic gout. Arthritis Rheum. 2002;47(4):356-60.

38. Perez-Ruiz F, Moreno-Lledó A, Urionagüena I, Dickson AJ. Treat to target in gout. Rheumatology. 2018;57(1):i20-6.

39. Scheepers LEJM, van Onna M, Stehouwer CDA, Singh JA, Arts ICW, Boonen A. Medication adherence among patients with gout: A systematic review and meta-analysis. Semin Arthritis Rheum. 2018;47(5):689-702.

40. Brown MT, Bussell JK. Medication adherence: WHO cares? Mayo Clin Proc. 2011;86(4):304-14.

41. Cramer JA, Roy A, Burrell A, Fairchild CJ, Fuldeore MJ, Ollendorf DA, et al. Medication compliance and persistence: Terminology and definitions. Value Heal. 2008;11(1):44-7.

42. Perez-Ruiz F, Desideri G. Improving adherence to gout therapy: An expert review. Ther Clin Risk Manag. $2018 ; 14: 793-802$

43. Wolfe F, Pincus T. Listening to the patient: A practical guide to self-report questionnaires in clinical care. Arthritis Rheum. 1999;42(9):1797-808.

44. Tatlock S, Rüdell K, Panter C, Arbuckle R, Harrold LR, Taylor WJ, et al. What Outcomes are Important for Gout Patients? In-Depth Qualitative Research into the Gout Patient Experience to Determine Optimal Endpoints for Evaluating Therapeutic Interventions. Patient. 2017:10(1):65-79.

45. Lindsay K, Gow P, Vanderpyl J, Logo P, Dalbeth N. The experience and impact of living with gout: A study of men with chronic gout using a qualitative grounded theory approach. J Clin Rheumatol. 2011;17(1):1-6

46. Singh JA. The impact of gout on patient's lives: a study of African-American and Caucasian men and women with gout. Arthritis Res Ther. 2014;16(3):R132.

47. Ellwood PM. Shattuck Lecture - Outcomes management A Technology of Patient Experience. N Engl J Med. 1988;318(23):1549-56.

48. McHorney CA. Health Status Assessment Methods for Adults: Past Accomplishments and Future Challenges. Annu Rev Public Health. 1999;20:309-35.

49. Demuro C, Clark M, Doward L, Evans E, Mordin M, Gnanasakthy A. Assessment of PRO label claims granted by the FDA as compared to the EMA (2006-2010). Value Heal. 2013;16(8):1150-5.

50. Fries J, Spitz P, Young D. The dimensions of health outcomes: the health assessment questionnaire, disability and pain scales. J Rheumatol. 1982;9(5):789-93.

51. Callahan LF. The History of Patient-Reported Outcomes in Rheumatology. Rheum Dis Clin North Am. 2016;42(2):205-17.

52. Pincus T, Yazici Y, Bergman MJ. Patient Questionnaires in Rheumatoid Arthritis: Advantages and Limitations as a Quantitative, Standardized Scientific Medical History. Rheum Dis Clin North Am. 2009;35(4):735-43.

53. McCollum L, Pincus T. A Biopsychosocial Model to Complement a Biomedical Model: Patient Questionnaire Data and Socioeconomic Status Usually Are More Significant than Laboratory Tests and Imaging Studies in Prognosis of Rheumatoid Arthritis. Rheum Dis Clin North Am. 2009;35(4):699-712.

54. Verhoeven AC, Boers M, Linden S Van Der. Responsiveness of the core set, response criteria, and utilities in 
early rheumatoid arthritis. Ann Rheum Dis. 2000;59(12):966-74.

55. Boonen A, Stucki G, Maksymowych W, Rat AC, Escorpizo R, Boers M, et al. The OMERACT-ICF reference group: Integrating the ICF into the OMERACT process: Opportunities and challenges. J Rheumatol. 2009;36(9):2057-60.

56. International Classification of Functioning, Disability and Health. Geneva: World Health Organization; 2001.

57. Oude Voshaar MA., Das Gupta Z, Bijlsma JW, Boonen A, Chau J, Courvoisier DS, et al. The International Consortium for Health Outcome Measurement (ICHOM) Set of Outcomes that Matter to People Living with Inflammatory Arthritis Consensus from an international Working Group. Arthritis Care Res. 2019; [Epud ahead of print].

58. Grainger R, Taylor WJ, Dalbeth N, Perez-Ruiz F, Singh JA, Waltrip RW, et al. Progress in measurement instruments for acute and chronic gout studies. J Rheumatol. 2009:36(10):2346-55.

59. Chandratre P, Roddy E, Clarson L, Richardson J, Hider SL, Mallen CD. Health-related quality of life in gout: a systematic review. Rheumatology. 2013;52(11):2031-40

60. Khanna PP, Nuki G, Bardin T, Tausche AK, Forsythe A, Goren A, et al. Tophi and frequent gout flares are associated with impairments to quality of life, productivity, and increased healthcare resource use: Results from a cross-sectional survey. Health Qual Life Outcomes. 2012;10:1-11.

61. Schumacher HR, Taylor W, Edwards L, Grainger R, Schlesinger N, Dalbeth N, et al. Outcome domains for studies of acute and chronic gout. J Rheumatol. 2009;36(10):2342-5.

62. Rai SK, Burns LC, De Vera MA, Haji A, Giustini D, Choi HK. The economic burden of gout: A systematic review. Semin Arthritis Rheum. 2015;45(1):75-80.

63. Jackson R, Shiozawa A, Buysman EK, Altan A, Korrer S, Choi H. Flare frequency, healthcare resource utilisation and costs among patients with gout in a managed care setting: a retrospective medical claimsbased analysis. BMJ Open. 2015;5(6):e007214

64. Edwards NL, Sundy JS, Forsythe A, Blume S, Pan F, Becker MA. Work productivity loss due to flares in patients with chronic gout refractory to conventional therapy. J Med Econ. 2011;14(1):10-5.

65. World Health Organization. Making choices in health: WHO Guide to Cost-Effectiveness Analysis. Edejer T, Baltussen R, Adam T, Hutubessy R, Acharya A, Evans D, et al., editors. World Health Organization; 2003. $318 \mathrm{p}$.

66. Keenan RT, O'Brien WR, Lee KH, Crittenden DB, Fisher MC, Goldfarb DS, et al. Prevalence of contraindications and prescription of pharmacologic therapies for gout. Am J Med. 2011;124(2):155-63.

67. Pascart T, Lioté F. Gout: state of the art after a decade of developments. Rheumatology. 2019;58(1):27-44.

68. Nocturne G, Ora J, Ea H-K, Lioté F. Influenza A H1N1 and anakinra exposure in a patient with gout. Jt Bone Spine. 2010;77(4):369-7.

69. Avram A, Duarte C, José Santos M, Papagoras C, Ritis K, Scarpioni R, et al. Identifying Patient Candidates for IL-1 Inhibition: Lessons From Real-World Cases. Jt Bone Spine 82. 2015; eS17-eS29.

70. Chen K, Fields T, Mancuso CA, Bass AR, Vasanth L. Anakinra's Efficacy is Variable in Refractory Gout: Report of Ten Cases. Semin Arthritis Rheum. 2010;40(3):210-4.

71. Thueringer JT, Doll NK, Gertner E. Anakinra for the treatment of acute severe gout in critically ill patients. 
Semin Arthritis Rheum. 2015;45(1):81-5.

72. Gratton SB, Scalapino KJ, Fye KH. Case of anakinra as a steroid-sparing agent for gout inflammation. Arthritis Care Res. 2009;61(9):1268-70.

73. McGonagle D, Tan AL, Shankaranarayana S, Madden J, Emery P, McDermott MF. Management of treatment resistant inflammation of acute on chronic tophaceous gout with anakinra. Ann Rheum Dis. 2007;66(12):16834.

74. Singh D, Huston KK. IL-1 Inhibition With Anakinra in a Patient With Refractory Gout. J Clin Rheumatol. 2009:15(7):366

75. Ghosh P, Cho M, Rawat G, Simkin PA, Gardner GC. Treatment of acute gouty arthritis in complex hospitalized patients with anakinra. Arthritis Care Res. 2013;65(8):1381-4.

76. Ottaviani S, Moltó A, Ea H-K, Neveu S, Gill G, Brunier L, et al. Efficacy of anakinra in gouty arthritis: a retrospective study of 40 cases. Arthritis Res Ther. 2013;15(5):R123.

77. So A, De Smedt T, Revaz S, Tschopp J. A pilot study of IL-1 inhibition by anakinra in acute gout. Arthritis Res Ther. 2007;9(2):R28.

78. Vitale A, Cantarini L, Rigante D, Bardelli M, Galeazzi M. Anakinra treatment in patients with gout and type 2 diabetes. Clin Rheumatol. 2015;34(5):981-4.

79. Tran AP, Edelman J. Interleukin-1 inhibition by anakinra in refractory chronic tophaceous gout. Int J Rheum Dis. 2011;14(3):33-7.

80. Aouba A, Deshayes S, Frenzel L, Decottignies A, Pressiat C, Bienvenu B, et al. Efficacy of anakinra for various types of crystal-induced arthritis in complex hospitalized patients: a case series and review of the literature. Mediators Inflamm. 2015;2015:7.

81. Petite SE. Effectiveness of Anakinra in Acute Gout: A Retrospective Review of Initial and Refractory Therapy. Am J Ther. 2017;24(5):e633-34.

82. Van Wabeke J, Dhondt E, Peene I, Piette Y. Anakinra in resistant gout: a case report. Acta Clin Belg. $2016 ; 72(4): 293-5$.

83. So A, Dumusc A, Nasi S. The role of IL-1 in gout: from bench to bedside. Rheumatology. 2018;57:i12-9.

84. Hui M, Carr A, Cameron S, Davenport G, Doherty M, Forrester H, et al. The British Society For Rheumatology guideline for the management of gout. Rheumatology. 2017;56(7):e1-20.

85. Becker MA, MacDonald PA, Hunt BJ, Lademacher C, Joseph-Ridge N. Determinants of the clinical outcomes of gout during the first year of urate-lowering therapy. Nucleosides, Nucleotides and Nucleic Acids. 2008;27(6-7):585-91.

86. Wortmann RL, MacDonald PA, Hunt B, Jackson RL. Effect of Prophylaxis on Gout Flares After the Initiation of Urate-Lowering Therapy: Analysis of Data From Three Phase III Trials. Clin Ther. 2010;32(14):2386-97.

87. Rashid N, Levy GD, Wu Y-L, Zheng C, Koblick R, Cheetham TC. Patient and clinical characteristics associated with gout flares in an integrated healthcare system. Rheumatol Int. 2015;35(11):1799-807.

88. Halpern R, Fuldeore MJ, Mody RR, Patel PA, Mikuls TR. The effect of serum urate on gout flares and their associated costs: An administrative claims analysis. J Clin Rheumatol. 2009;15(1):3-7.

89. Wu E, Patel P, Mody R, Yu A, Cahill K, Tang J, et al. Frequency, risk, and cost of gout-related episodes among 
the elderly: does serum uric acid level matter? J Rheumatol. 2009;36(5):1032-40.

90. Kiltz U, Smolen J, Bardin T, Cohen Solal A, Dalbeth N, Doherty M, et al. Treat-to-target (T2T) recommendations for gout. Ann Rheum Dis. 2017;76(4):632-8.

91. Taylor WJ, Redden D, Dalbeth N, Schumacher HR, Edwards NL, Simon LS, et al. Application of the OMERACT filter to measures of core outcome domains in recent clinical studies of acute gout. J Rheumatol. 2014; 41(3):574-80.

92. Mokkink LB, Terwee CB, Patrick DL, Alonso J, Stratford PW, Knol DL, et al. The COSMIN study reached international consensus on taxonomy, terminology, and definitions of measurement properties for healthrelated patient-reported outcomes. J Clin Epidemiol. 2010;63(7):737-45.

93. Mokkink LB, Terwee CB, Patrick DL, Alonso J, Stratford PW, Knol DL, et al. The COSMIN checklist for assessing the methodological quality of studies on measurement properties of health status measurement instruments: An international Delphi study. Qual Life Res. 2010;19(4):539-49.

94. Gandhi PK, Gentry WM, Ma Q, Bottorff MB. Cost-effectiveness analysis of allopurinol versus febuxostat in chronic gout patients: a U.S. payer perspective. J Manag care Spec Pharm. 2015;21(2):165-75.

95. Beard SM, von Scheele BG, Nuki G, Pearson I V. Cost-effectiveness of febuxostat in chronic gout. Eur J Heal Econ. 2014;15(5):453-63.

96. Liu X, Sun D, Ma X, Li C, Ying J, Yan Y. Benefit-risk of corticosteroids in acute gout patients: An updated meta-analysis and economic evaluation. Steroids. 2017;128:89-94

97. Jutkowitz E, Choi HK, Pizzi LT, Kuntz KM. Cost-effectiveness of allopurinol and febuxostat for the management of gout. Ann Intern Med. 2014;161(9):617-26.

98. Cattermolea GN, Man CY, Cheng CH, Graham CA, Rainera TH. Oral prednisolone is more cost-effective than oral indomethacin for treating patients with acute gout-like arthritis. Eur J Emerg Med. 2009;16(5).

99. Meltzer M, Pizzi LT, Jutkowitz E. Payer decision-making with limited comparative and cost effectiveness data: the case of new pharmacological treatments for gout. Evid Based Med. 2012;17(4):105-8.

100. Perez-Ruiz F, Díaz-Torné C, Carcedo D. Cost-effectiveness analysis of febuxostat in patients with gout in Spain. J Med Econ. 2016;19(6):604-10.

101. Smolen LJ, Gahn JC, Mitri G, Shiozawa A. Febuxostat in the management of gout: a cost-effectiveness analysis. J Med Econ. 2016;19(3):265-76.

102. Ruggeri M, Basile M, Drago C, Rolli FR, Cicchetti A. Cost-Effectiveness Analysis of Lesinurad/Allopurinol Versus Febuxostat for the Management of Gout/Hyperuricemia in Italy. Pharmacoeconomics. 2018;36(5):625-36. 



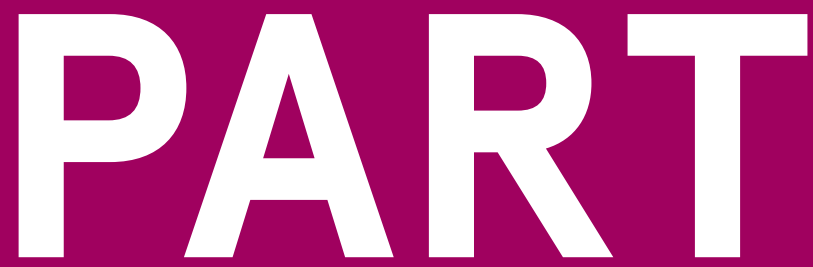

Clinical management of gout and hyperuricemia 


\section{Anakinra for the treatment of acute gout flares: \\ a randomized, double-blind, \\ placebo-controlled, \\ active-comparator, \\ non-inferiority trial}

Carly A. Janssen, Martijn A.H. Oude Voshaar, Harald E. Vonkeman, Tim L.Th.A. Jansen, Matthijs Janssen, Marc R. Kok, Bea Radovits, Caroline van Durme, Hetty Baan, Mart A.F.J. van de Laar 


\section{ABSTRACT}

Objectives. To evaluate the efficacy and safety of anakinra in treating acute gout flares in a randomized, double-blind, placebo-controlled, active comparator, non-inferiority (NI) trial.

Methods. Patients with a crystal-proven acute gout flare were randomized (1:1) to treatment with anakinra or treatment as usual (free choice: either colchicine, naproxen or prednisone). The primary end point was the change in pain between baseline and the averaged pain score on days 2-4 measured on a five-point rating scale. $\mathrm{NI}$ of anakinra would be established if the upper bound of the $95 \%$ confidence interval (Cl) of the numeric difference in changed pain scores between treatment groups did not exceed the $\mathrm{NI}$ limit of 0.4 in favour of treatment as usual, in the per-protocol (PP) and intentionto-treat (ITT) populations, assessed in an analysis of covariance model. Secondary outcomes included safety assessments, improvement in pain, swelling, tenderness and treatment response after 5 days, assessed using linear mixed models and binary logistic regression models.

Results. Forty-three patients received anakinra and 45 treatment as usual. Anakinra was non-inferior (mean difference; $95 \% \mathrm{Cl}$ ) to treatment as usual in both the PP $(-0.13$; $-0.44,0.18)$ and ITT $(-0.18 ;-0.44,0.08)$ populations. No unexpected or uncommon (serious) adverse events were observed in either treatment arm. Analyses of secondary outcomes showed that patients in both groups reported similar significant reductions in their gout symptoms.

Conclusion. Efficacy of anakinra was shown to be non-inferior to treatment as usual for the treatment of acute gout flares, suggesting that anakinra is an effective treatment alternative for acute gout flares.

Trial registration. Het Nederlands Trial Register, www.trialregister.nl, NTR5234

\section{Key Messages.}

- A five-day treatment of anakinra was non-inferior to registered treatment as usual in treating acute gout flares.

- Results suggest anakinra is an effective treatment alternative for the treatment of acute gout flares. 


\section{INTRODUCTION}

Gout is a common form of auto-inflammatory arthritis, caused by the deposition of MSU crystals within the soft tissue of synovial joints ${ }^{[1]}$. Acute gout flares are characterized by distinct inflammatory symptoms (e.g. pain, erythema, swelling), and may cause physical disability, as well as decreased quality of life ${ }^{[2,3]}$. Therefore, rapid reduction of autoinflammation to achieve prompt symptom control is a main goal in managing acute gout.

Colchicine, NSAIDs and glucocorticosteroids are recommended first-line treatment agents for acute gout ${ }^{[4-8]}$. Unfortunately, comorbidities that may result in contraindications to these medications are common in gout ${ }^{[9,10]}$. Moreover, some patients are intolerant of, or fail to respond to these medications [11]. For such patients, potent treatment alternatives are needed. The 2016 updated EULAR guidelines recommend considering treatment with an IL-1 inhibitor in patients having frequent flares and in those who are difficult to treat using conventional therapies ${ }^{[5]}$.

Canakinumab is currently the only IL-1 inhibitor that has been registered in Europe for the treatment of gout. However, its high costs per treatment may discourage prescribers in daily practice. An alternative might be the IL-1 receptor antagonist anakinra ${ }^{[12]}$. Although anakinra has been applied in rheumatoid arthritis for many years, trials on its efficacy and safety in acute gout are lacking, having only been investigated in case reports, a few retrospective studies and a small open-label study ${ }^{[13-29]}$. Therefore, we evaluated the efficacy and safety of anakinra for the treatment of acute gout flares, compared with treatment as usual, in a randomized, controlled, non-inferiority (NI) trial.

\section{METHODS}

\section{Study design and patient population}

This randomized, double-blind, double-dummy, active comparator placebo-controlled trial, was conducted at the rheumatology departments of seven hospitals in the Netherlands, between 2016 and 2018. The study was approved by an Ethical Review Board (METC Twente, Enschede, the Netherlands), the institutional review board or ethics committee of each participating centre, and performed in accordance with the principles outlined in the Declaration of Helsinki and Dutch legislation.

Eligible subjects were recruited and screened by the attending rheumatologist and an instructed specialized rheumatology nurse at the outpatient clinics. All adult $(\geq 18$ 
years) patients, with a diagnosis of an acute flare of gouty arthritis, confirmed by microscopic identification of intracellular MSU crystals in the primary joint were eligible for participation. The primary joint was defined as the joint that was most affected by acute gouty arthritis, according to the rheumatologist. Patients with current use of urate lowering therapy (ULT), as well as those experiencing only no to mild gout-related pain, were not included. Other non-inclusion criteria included concurrent use of other IL-1 inhibitors; known history of allergy or sensitivity to latex; absolute contraindication for all available types of ULT; absolute contraindication to anakinra (e.g. neutropenia and severe renal impairment defined as a creatinine clearance rate $<30 \mathrm{ml} / \mathrm{min}$ ); absolute contraindication for all three conventional treatment options; presence of liver disease that according to the treating rheumatologist precluded participation in the study; an active or recurrent bacterial, fungal or viral infection; use of tumour necrosis factor inhibitors; pregnancy or lactation; women who planned on becoming pregnant during the study period, and insufficient command of the Dutch language. Following screening, all patients were given a maximum of $24 \mathrm{~h}$ to decide on participation in the study and to provide written informed consent prior to randomization and study initiation at baseline.

\section{Randomization, treatment and dosing}

The attending rheumatologist, together with the patient, decided on the treatment as usual (colchicine, naproxen or prednisone) that would suit the patient best. Subsequently, patients were randomly allocated (1:1) to either a five-day treatment with anakinra (subcutaneous injection $100 \mathrm{mg}$ once daily) plus oral placebo up to three times daily (colchicine), two times daily (naproxen) or one time daily (prednisone); or to a treatment with oral standard of care in line with the assigned standard treatment by the caregiver $10.5 \mathrm{mg}$ up to three times daily for colchicine; $500 \mathrm{mg}$ up to twice daily for naproxen; $35 \mathrm{mg}$ once daily for 5 days for prednisone) plus subcutaneous injection placebo once daily for 5 days. For treatment as usual, the dosages and duration were in line with national acute gout treatment guidelines ${ }^{[4]}$. The placebo injections and placebo pills were identical in appearance to the anakinra injections and treatment as usual pills, respectively. Considering the short duration of anakinra treatment given to patients, no pre-study screening for latent tuberculosis was done. The randomization allocation sequence list was generated using a computer randomization application, based on atmospheric noise. Patients, caregivers, local pharmacies, and trial investigators had no knowledge of the allocation sequence during the entire course of the study. Study medication was stored at the hospital pharmacy and released in sequential order to patients. Patients received instructions on the use of study medication from a blinded study nurse, and the first dosages of study medication, both oral pills and injection, were taken by patients under supervision of a blinded study nurse during the baseline visit. During the first 7 days of 
the study, no prescription-based, rescue medication was available to patients, but the use of over-the-counter pain-relieving agents (NSAIDs and aspirin) was allowed. Patients also initiated ULT at baseline with allopurinol, febuxostat or benzbromarone, at the discretion of the treating rheumatologist.

\section{Study assessments}

For this study, the OMERACT recommendations for acute gout were followed ${ }^{[30]}$. Starting at baseline (day 1), patients were asked to fill in a gout flare diary for seven consecutive days, wherein levels of pain ( $1=$ none; 2 = mild; 3 = moderate; 4 = a lot; 5 = extreme), tenderness ( 1 = none; 2 = slightly; 3 = fairly; 4 = very; 5 = extremely) and swelling $(1=$ none; 2 = somewhat; 3 = fairly; 4 = very; 5 = extremely $)$ of the primary joint were recorded using five-point rating scales. Patients were also asked to report their level of pain in the primary joint ( 0 = absolutely no pain, $10=$ unbearable pain) and their global assessment of overall wellbeing ( 0 = very bad, 10 = very good) on a 10 -point numeric rating scale (NRS), and their level of treatment response on an eight-point rating scale (1 = completely disappeared; 2 = very much improved; 3 = much improved; 4 = somewhat improved; 5 = unchanged; 6 = slightly worse; 7 = much worse; 8 = very much worse). Finally, experienced side-effects and the intake of any other painkillers and/or antiinflammatory medication were to be reported in the flare diary daily.

Physical examination, medical history and gout status were assessed at baseline, and patients returned for a clinic visit at day 7. Laboratory measurements (serum uric acid, $c$-reactive protein (CRP)) were performed at baseline and at day 7. Any (serious) adverse events ((S)AE)) were reported and evaluated at day 7 .

\section{Study endpoints}

The primary end point, i.e. $\Delta$ Pain, was defined as the mean change in patient-reported pain in the most affected joint, from baseline to the average of pain scores at days 2-4 on the five-point rating scale ${ }^{[31,32]}$. We reasoned a priori that anakinra could be a useful treatment alternative for gout patients with contraindications to the standard treatment options, even when not proven more effective than treatment as usual, if it could be demonstrated that anakinra has an effect greater than the effect of imputed placebo [33]; and that any potential inferiority of anakinra compared with treatment as usual would not be clinically meaningful. Therefore, a NI design was adopted with a NI margin of 0.4 points on the five-point rating scale. A difference of 0.4 points in favour of treatment as usual is slightly more stringent compared with previous $\mathrm{NI}$ studies in gout known to us, that have used 0.5 as a $\mathrm{NI}$ margin for $\triangle \mathrm{Pain}{ }^{[31,34,35]}$. It is also more stringent than other studies assessing change in pain as a primary outcome, for which $10 \%$ of the 
scale (e.g. $10 \mathrm{~mm}$ on a 100-mm visual analogue scale (VAS)) is frequently used as an upper limit of acceptable difference ${ }^{[36,37]}$. Moreover, in the only available study that allowed for a placebo effect to be estimated for $\triangle$ Pain, the difference between groups in $\triangle$ Pain was found to be at least 0.4 points in favour of NSAID compared with placebo (i.e. $95 \% \mathrm{Cl}$ was 0.4-1.0) ${ }^{[32]}$. Therefore, assuming a placebo effect of similar magnitude, the effect of anakinra in the current trial can be considered greater than imputed placebo when the upper bound of the $95 \% \mathrm{Cl}$ for the $\Delta$ Pain (anakinra-treatment as usual) does not exceed

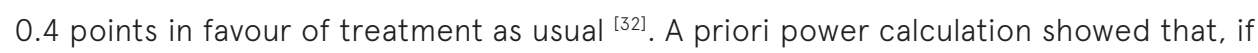
there would be no difference in $\triangle$ Pain between the groups, 87 patients per treatment arm would need to be included to have an $80 \%$ chance of demonstrating $\mathrm{NI}$ with a chance of a type 1 error of $5 \%$ or less.

Secondary outcomes included the improvement of primary joint pain (NRS scores), tenderness, swelling, treatment response and patient global assessment (PGA) of wellbeing across days $1-5$. The number of patients achieving $\geq 50 \%$ decrease in NRS pain scores following baseline on days 2-5 were also compared between treatment arms. Other outcomes included the number and type of $A E$ that occurred during the first 7 days of the study, as well as the decrease in CRP levels after 7 days. Finally, use of concomitant pain-relieving agents, both prescription-based and over the counter medication, during days 1-7 were determined.

\section{Statistical analysis}

The primary study end point was assessed using an analysis of covariance (ANCOVA) model, with treatment received as a fixed effect, and baseline pain scores measured on the five-point rating scale as a covariate. Subsequently, we obtained a $95 \% \mathrm{Cl}$ for the baseline pain adjusted marginal mean difference in $\triangle$ Pain between the treatment groups. We tested whether the upper bound of the $\mathrm{Cl}$ of the numeric difference in changed pain scores between anakinra and treatment as usual would not exceed the $\mathrm{NI}$ margin of 0.4 in favour of treatment as usual ( $\mathrm{HO}: \Delta \mathrm{Pain}_{\text {Anakinra }}-\Delta \mathrm{Pain}_{\text {TreatmentAsUsual }}>0.4 \mathrm{vs}$. Ha: $\triangle$ Pain $_{\text {Anakinra }}-\triangle P$ Pain $\left._{\text {TreatmentAsUsual }} \leq 0.4\right)$. If this criterion was met in both the per-protocol (PP) and intention-to-treat (ITT) populations, NI of anakinra compared with treatment as usual would be concluded. The ITT analysis was performed on all patients who were randomized to a treatment at baseline and received at least one dose of study medication. The PP population contained patients who had no missing data for $\geq 1$ of the assessments needed for the primary outcome, and who did not take any interfering concomitant painrelieving medications during days $1-4$ of the study. This included any prescription-based pain-relieving medication (e.g. opioid formulations, intake of colchicine while the patient was prescribed naproxen), but over the counter pain-relieving agents were allowed. 
Analysis of secondary endpoints were performed in the ITT population, using linear mixed effects models, with time, treatment arm, and the interaction between time and treatment arm as fixed effects. For each outcome analysed, the covariance matrix was chosen that had the best fit according to the Bayesian information criterion. All statistical tests were performed at the Bonferroni corrected 0.05 level. The difference between treatment groups in achieving $\geq 50 \%$ decrease in NRS pain scores in the days following baseline was assessed using binary logistic regression analysis.

For the analyses of the ITT population, plausible values for missing observations of the seven-day flare diary and CRP values were generated using multiple imputation by chained equations. Percentages of missing data were determined for variables needed for the primary efficacy analyses. Data from the seven-day flare diary was used as input for the imputation models, as well as treatment received, age, gender and logtransformed CRP values. Following the imputation process, the latter were transformed back to normal CRP values. In total, 20 datasets were generated, and the observed and imputed distributions were visually compared for similarity. Reporting guidelines for handling analysis affected by missing data were followed ${ }^{[38]}$.

Characteristics of the study population were summarized using means and standard deviations, or median and first and third quartiles for continuous outcomes. Categorical variables were summarized using frequency counts and percentages, and compared using Pearson's $X^{2}$ statistics, as appropriate, for secondary outcomes. Other secondary outcomes were compared using t-tests or a non-parametric equivalent, as appropriate. $P$-values $<0.05$ were considered statistically significant. All analyses were done using IBM statistics SPSS version 22. The Reporting of $\mathrm{NI}$ and Equivalence Randomized Trials extension of the CONSORT 2010 statement was followed in reporting this study ${ }^{\text {[39]. }}$

\section{RESULTS}

\section{Patient characteristics}

Eighty-eight patients were enrolled in the study, and all patients $(100 \%)$ completed the seven-day follow-up. Of these, 43 patients (48.9\%) were randomized to a treatment with anakinra and 45 (51.1\%) to treatment as usual (Fig. 1). Patient baseline characteristics are listed in Table 1. The majority of patients in both groups (>90\%) were male and more than half of the patients had monoarticular gout. Of the patients in the treatment as usual arm, 18 received a treatment with colchicine, and 13 and 14 patients received a treatment with naproxen and prednisone, respectively. 


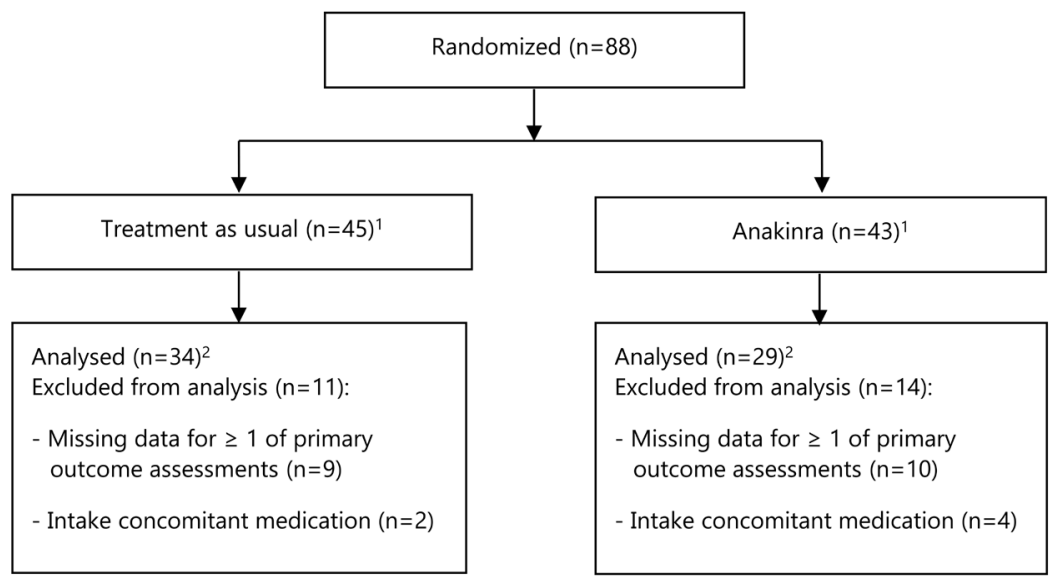

Fig. 1 Flow diagram of patient selection

${ }^{1}$ Intention-to-treat population, ${ }^{2}$ Per-protocol population.

Table 1: Baseline demographic and clinical characteristics

\begin{tabular}{llll}
\hline & $\begin{array}{l}\text { Score range } \\
\text { of measure }\end{array}$ & $\begin{array}{l}\text { Treatment as usual } \\
(\mathbf{n}=\mathbf{4 5})\end{array}$ & $\begin{array}{l}\text { Anakinra } \\
(\mathbf{n}=\mathbf{4 3})\end{array}$ \\
\hline Age, years, mean \pm S.D. & - & $59.9 \pm 12.7$ & $63.4 \pm 12.9$ \\
Male sex & - & $42(93.3)$ & $41(95.3)$ \\
BMI, kg/m², mean \pm S.D. & - & $28.6 \pm 4.0$ & $29.5 \pm 4.2$ \\
Systolic blood pressure, mm Hg, mean \pm S.D. & $0-999$ & $141.5 \pm 24.2$ & $145.1 \pm 21.6$ \\
Diastolic blood pressure mm Hg, mean \pm S.D. & $0-999$ & $85.7 \pm 14.6$ & $84.8 \pm 14.6$ \\
SUA, mmol/L, median (Q1, Q3) & $0-9$ & $0.52(0.45,0.64)$ & $0.50(0.42,0.57)$ \\
CRP, mg/L, median (Q1, Q3) & $0-999$ & $14.0(6.0,30.5)$ & $15.0(5.5,32.0)$ \\
Comorbidities & & & \\
$\quad$ Hypertension & - & $17(65)$ & $10(37)$ \\
$\quad$ Cardiovascular disease & - & $11(42)$ & $18(67)$ \\
$\quad$ Diabetes Mellitus & - & $5(19)$ & $3(11)$ \\
$\quad$ Renal disorders & - & $1(4)$ & $4(15)$ \\
$\quad$ Musculoskeletal disease ${ }^{c}$ & - & $4(15)$ & $6(22)$ \\
Gout classification $^{d}$ & - & & $23(53.5)$ \\
$\quad$ Monoarticular & - & $30(66.7)$ & $17(39.5)$ \\
$\quad$ Oligoarticular & - & $11(24.4)$ & $3(7.0)$ \\
\hline Polyarticular & - & $4(8.9)$ & \\
\hline
\end{tabular}

Data are presented as $\mathrm{n}(\%)$ unless stated otherwise. ${ }^{a}$ In the treatment as usual group, 18 patients received colchicine, 13 naproxen and 14 prednisone. ${ }^{b}$ Number and percentages based on available data from patients in the anakinra $\operatorname{arm}(n=27)$ and treatment as usual $\operatorname{arm}(n=26) .{ }^{c}$ Diseases other than gout. ${ }^{d}$ Monoarticular implies one joint has been affected by gout; oligoarticular $>1$ but $<5$ joints have been affected by gout; polyarticular $\geq 5$ joints have been affected by gout. SUA: serum urate acid. 


\section{Efficacy assessment}

Pain scores measured on the five-point rating scale decreased to a similar extent in both groups over days 1-4. For the PP population, results of the univariate ANCOVA for the primary outcome showed that the estimated marginal mean difference between treatment arms, -0.132 points on the five-point rating scale, was in favour of anakinra, and that the upper bound of the $95 \% \mathrm{Cl}$ of this difference $(-0.44,0.18)$ did not surpass the NI margin of 0.4 (Fig. 2). In the ITT population, plausible values for missing data of the five-point rating scale pain scores at baseline, day 2, day 3 , and day 4 post baseline were generated for $12(13.6 \%), 6(6.8 \%), 3(3.4 \%)$ and $5(5.7 \%)$ cases, respectively. Here, the primary analysis yielded similar results, with an estimated marginal mean difference of $-0.178(95 \% \mathrm{Cl}-0.44,0.08)$ (Fig. 2). Because in both of these analyses the upper bound of the $95 \% \mathrm{Cl}$ did not surpass the $0.4 \mathrm{NI}$ margin, the null hypothesis that treatment with anakinra is less effective than treatment as usual for treating acute gout flares by at least 0.4 points on the five-point rating scale, was rejected. We concluded that the pain-relieving effect of anakinra in patients with gout was greater than the estimated effect of placebo, and that efficacy of anakinra was non-inferior to treatment as usual.

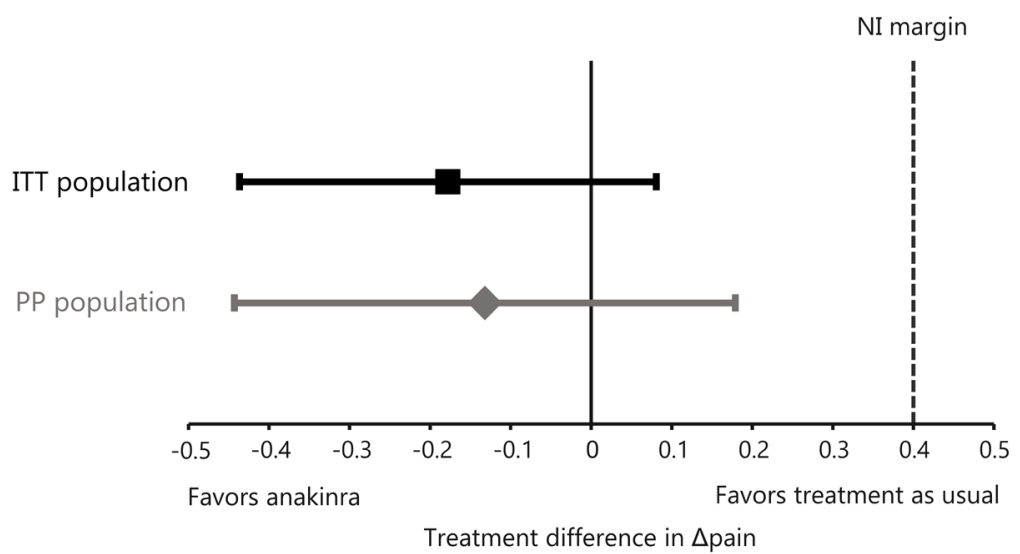

Fig. 2 The $95 \% \mathrm{Cl}$ for the estimated marginal mean difference in $\Delta$ Pain between the treatment groups

$\Delta$ Pain is the mean change in patient-reported pain from baseline to the average of pain scores at days 2-4 on the five-point rating scale. NI: non-inferiority.

\section{Secondary outcomes}

For all the secondary outcomes assessed using the linear mixed effects model, the pattern of change was similar for the anakinra and treatment as usual group over 5 days, with significant improvements over time, but no significant between-group effects, or group- 
by-time interactions (Fig. 3; results for PGA not shown). These findings provide no evidence for a differential effect on any outcome. For PGA, the mean \pm standard error (S.E.) scores increased from $4.4 \pm 0.34$ to $6.7 \pm 0.31$ in the conventional treatment arm, and from 4.9 \pm 0.38 to $7.31 \pm 0.32$ in the anakinra treatment group. After 2 days, more patients in the anakinra treatment group achieved $\geq 50 \%$ decrease in NRS pain scores (odds ratio (OR) $1.41,95 \% \mathrm{Cl} 0.53,3.73)$ compared with the treatment as usual arm, although not statistically significant. Also, on days 3-5, OR were in favour for anakinra. However, only on day 3 was this difference statistically significant (Supplemental Material 1).
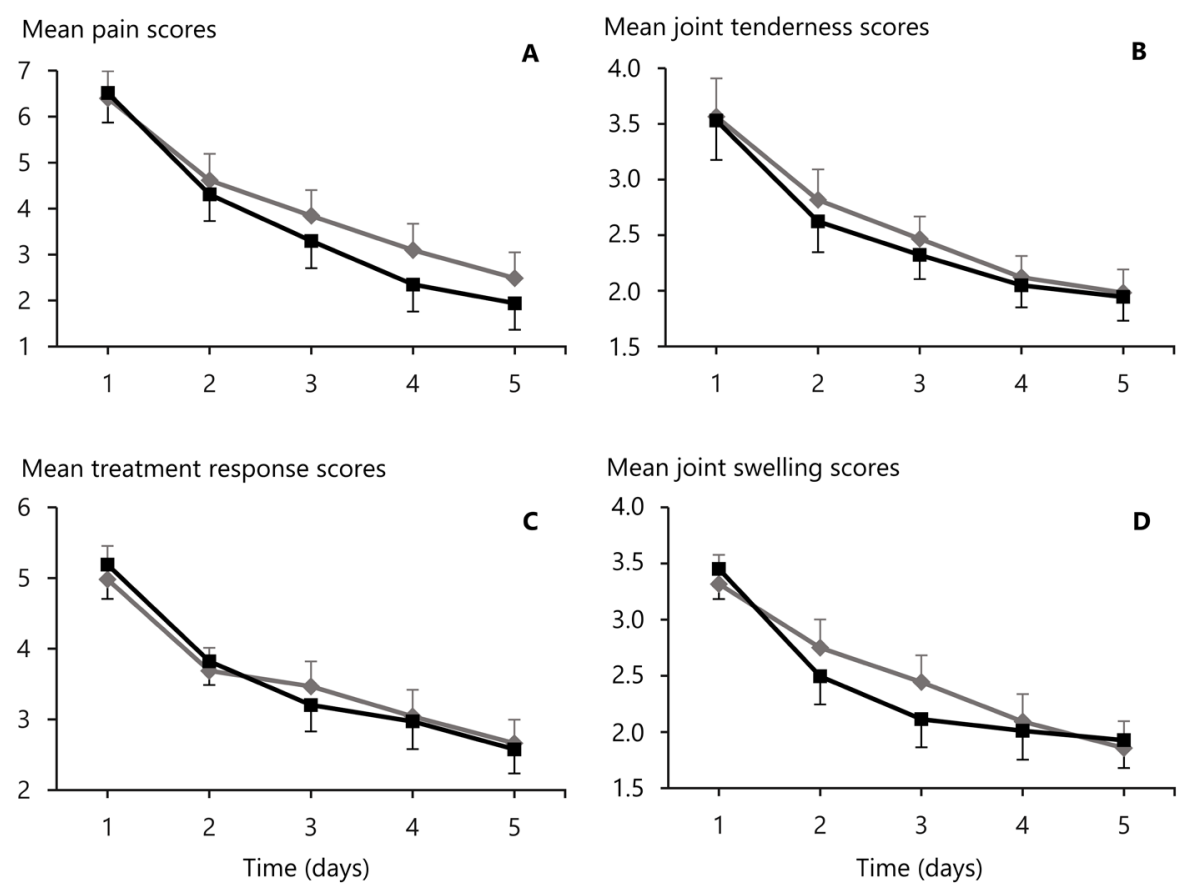

Mean joint swelling scores

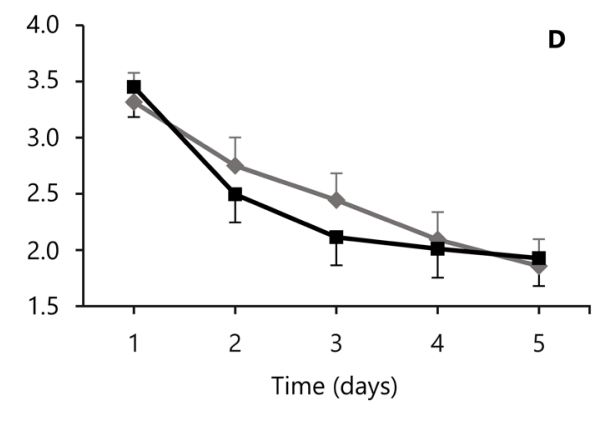

Anakinra

Treatment as usual

Fig. 3 Mean scores on days 1-5 (bars represent one-sided 95\% Cl) of the secondary outcomes (panel A-D)

Both treatment groups showed a reduction in their CRP values after 7 days from baseline. The mean \pm SE reduction in the treatment as usual arm was $13.9 \pm 7.1$ after 7 days, which was not significantly different to the reduction observed in the anakinra treatment arm of $11.1 \pm 7.5$. 
During the first 7 days following baseline, more patients in the anakinra group $(n=20$, $46.5 \%$ ) compared with the treatment as usual arm ( $n=16,35.6 \%$ ), took some form of pain-relieving medication, over the counter or prescription-based, aside from their study medication. However, this difference was not statistically significant between the groups.

\section{Safety assessment}

Out of the 88 patients, 36 (40.9\%) patients reported an AE during the first 7 days post baseline, of which more than half $(n=21,58.3 \%)$ were patients in the treatment as usual arm (Table 2). Interestingly, three injection site reactions were reported in the treatment as usual group with the placebo injections, compared with none in the anakinra treatment group. One patient in the treatment as usual group reported to have viral gastroenteritis (stomach flu), and one patient in the anakinra group had a respiratory tract infection (severe cold). No SAE were reported during the 7 days following baseline.

Table 2: Number and percentage (\%) of adverse events occurring per treatment arm during the 7 days following baseline.

\begin{tabular}{lll}
\hline & Treatment as usual (N=45) & Anakinra (N=43) \\
\hline Total number of SAEa reported & $0(0)$ & $0(0)$ \\
Number of cases having an AE ${ }^{\mathrm{b}}$ & $21(46.7)$ & $15(34.9)$ \\
Total number of AE reported & $49(57.0)$ & $37(43.0)$ \\
Type of AE reported $^{c}$ & & \\
$\quad$ Musculoskeletal Pain & $4(8.2)$ & $6(16.2)$ \\
Musculoskeletal stiffness, swelling, spasms & $4(8.2)$ & $5(13.5)$ \\
Injection site reaction $_{\text {Infections }}^{\text {d }}$ & $3(6.1)$ & $0(0.0)$ \\
Vomiting & $1(2.0)$ & $1(2.7)$ \\
Diarrhea & $0(0.0)$ & $1(2.7)$ \\
Nausea & $9(18.4)$ & $3(8.1)$ \\
Other Gl disorders & $5(10.2)$ & $3(8.1)$ \\
Headache & $3(6.1)$ & $0(0.0)$ \\
Dizziness & $4(8.2)$ & $2(5.4)$ \\
Fatigue & $4(8.2)$ & $0(0.0)$ \\
Pruritus (itchiness) & $1(2.0)$ & $2(5.4)$ \\
Paresthesia or tremor & $0(0.0)$ & $3(8.1)$ \\
Other AE & $1(2.0)$ & $2(5.4)$ \\
\hline
\end{tabular}

a Defined as any medical occurrence or event that was life threatening at the time of event, resulted in death, resulted in persistent or significant disability, led to hospitalization or prolongation of existing hospitalization, led to a congenital anomaly or birth defect. ${ }^{b}$ Given as percentage of total patients within group. ${ }^{c}$ Given as percentage of total number of AE reported within group. ${ }^{d}$ Type of infection reported in the treatment as usual group was gastroenteritis viral, and in the anakinra group a respiratory tract infection. SAE: serious adverse event; $\mathrm{AE}$ : adverse event; Gl: gastrointestinal. 


\section{DISCUSSION}

In this study we set out to determine the clinical efficacy and safety of anakinra for the treatment of acute, crystal-proven, gout flares in a randomized, active-comparator, $\mathrm{NI}$ trial. A daily subcutaneous injection of anakinra was compared with oral treatments administered according to the standard of care, using optimal dosages as recommended in gout guidelines. As far as we know, this is the first double-blind, randomized controlled trial to evaluate the use of anakinra in an acute gouty arthritis population.

The results presented here show at least $\mathrm{NI}$ of anakinra compared with conventional therapies for the treatment of acute gout flares, supported by the additional finding that patients in both treatment arms achieved a significant reduction of their goutrelated symptoms over the course of one week. Additionally, evaluation of the reported $A E$ did not reveal any severe, uncommon or unexpected safety outcomes. These findings suggest that anakinra might be a viable treatment option for gout flares.

To date, various non-randomized, uncontrolled, observational studies have reported on the clinical efficacy of anakinra for treating gout, mostly reporting promising outcomes on the numbers or percentages of patients who responded well to anakinra within the first days of treatment [15,16,29,40-43,17,22-28]. However, the absence of a control group in these studies hampers the interpretation of such results, as the observed improved clinical status of patients might merely reflect the self-limiting natural course of acute gout ${ }^{[44]}$. In our present study, anakinra was compared with treatment in accordance with current gout management guidelines, using several anti-inflammatory agents with previously established effectiveness in treating gout flares ${ }^{[5,45]}$. The results showed that the efficacy of anakinra in treating gout flares was consistently numerically superior to standard of care treatment across the primary and secondary study outcomes. However, the direct comparison of anakinra with standard of care does not by itself allow for conclusions about the effectiveness of either treatment in gout, as no placebo arm was included. By relating the results to a $\mathrm{NI}$ margin that was estimated using the imputed placebo method, our results indirectly support the efficacy of anakinra in gout. However, this conclusion is based on the assumption that the relative effectiveness of standard of care treatment compared with placebo would have been the same as the relative effectiveness of NSAID compared with placebo that was observed in the study used for estimating the NI margin ${ }^{[32,46,47]}$.

Data from our study builds on previous clinical trials that have reported varying efficacies of IL-1 inhibitors in gout patients. Terkeltaub et al. ${ }^{[32]}$ reported on rilonacept, 
in which treatment with rilonacept plus NSAID did not significantly improve pain levels compared with NSAID monotherapy, and rilonacept monotherapy was shown to be inferior to NSAID monotherapy over a 72-h follow-up period. This difference in efficacy compared with our study might be attributed to differences in study designs, but also to pharmacokinetic differences between the IL-1 inhibiting drugs. The authors reflect that rilonacept reaches its maximum plasma concentration after 48-72h, which could potentially have led to insufficient drug concentrations during the period of the primary outcome assessment. For anakinra, maximum plasma concentrations are reached within 3-7h. This rapid increase in drug plasma concentrations may explain why the results in our study seem more promising $72 \mathrm{~h}$ after baseline, than those seen with rilonacept. Canakinumab is currently the only IL-1 inhibitor for which effectiveness during an acute gout exacerbation has been demonstrated in a randomized study ${ }^{[36,48]}$. Schlesinger et al. ${ }^{[49]}$ reported that a single subcutaneous injection of canakinumab $150 \mathrm{mg}$ was superior to a single intramuscular injection of triamcinolone acetonide $40 \mathrm{mg}$, in relieving pain after $72 \mathrm{~h}$. The mean VAS $(0-100 \mathrm{~mm})$ pain score at that moment was $25 \mathrm{~mm}$ for the canakinumab arm. Although it is difficult to compare these outcomes with our results, because of differences in study design and populations, results from our study reveal similar pain scores after $72 \mathrm{~h}$ in the anakinra group on a 0-10 NRS (i.e. score $=2.4$ ).

Considering the high costs of canakinumab treatment, anakinra therefore seems to be a cost-effective alternative for gout patients seen in daily practice who are difficult to treat with conventional therapies, in case they meet the local reimbursement criteria. Importantly, anakinra could possibly allow a larger proportion of the difficult to treat gout population to be treated with an IL-1 inhibitor, including patients having comorbidities as diabetes mellitus, renal disorders or cardiovascular disease. In this respect it should be noted that patients with severe renal impairment were excluded from participation in our study. However, favourable outcomes have previously been described in a retrospective case-series of 31 patients with either a renal transplantation or stage 4 or stage 5 chronic kidney disease, that signs and symptoms of gout flares subsided after admission of treatment with anakinra ${ }^{[50]}$. Moreover, a current ongoing feasibility study, designed as a double-blind, double-dummy, randomized controlled trial, will contribute to developing a definite clinical trial wherein the efficacy and tolerability of anakinra to corticosteroids in patients with chronic kidney disease will be compared ${ }^{[20]}$.

The multicentre, randomized, placebo-controlled, double-blinded nature of our study, as well as the inclusion of only patients with MSU crystal-proven gout, the diagnostic gold-standard, are strengths of our study. However, the present study was not without limitations. First, the total number of patients included was lower than initially calculated 
in the power analyses for the primary efficacy analyses. However, in contrast to the a priori power calculation that assumed no difference between the treatment arms in $\triangle$ Pain, we found a difference in favour of anakinra in both the PP and ITT analyses, with on average 0.155 points on a five-point rating scale. Therefore, and having the other assumptions remain constant, a smaller sample size would have sufficed to demonstrate $\mathrm{NI}$ of anakinra compared with imputed placebo. Within the limitations of a small clinical trial, relatively few data on the safety of anakinra in gout was obtained. However, the long-term treatment experience of anakinra in rheumatoid arthritis already provides a sound safety profile. Nevertheless, additional short-term safety data for anakinra in gout is desired. Finally, the study was carried out in a hospital-based setting, possibly making our results less generalizable to a primary care setting, in which other types of acute gout patients may be treated.

In conclusion, in our study anakinra was shown to be non-inferior to registered treatment as usual for the treatment of acute gout flares. Our results suggest that anakinra is an effective treatment alternative for the treatment of acute gout flares.

\section{Acknowledgements}

We would like to thank all employees of the participating centres who helped in the selection of patients and data collection throughout the study; the employees at the dispensing pharmacies at each participating center in helping make this study feasible; the general practitioners in the region of Enschede who helped in the referral of potential subjects; and the patients who were willing to participate in this study. We also want thank Prof. Dr A. Boonen for her assistance in reviewing the final manuscript. 


\section{REFERENCES}

1. Cronstein BN, Esserman PR, Sunkureddi P. Mechanistic Aspects of Inflammation and Clinical Management of Inflammation in Acute Gouty Arthritis. J Clin Rheumatol. 2013;19(1):19-29.

2. Chandratre P, Roddy E, Clarson L, Richardson J, Hider SL, Mallen CD. Health-related quality of life in gout: a systematic review. Rheumatology. 2013;52(11):2031-40.

3. ten Klooster PM, Vonkeman HE, van de Laar MAFJ. Disability due to gouty arthritis. Curr Opin Rheumatol. 2012;24(2):139-44.

4. Nederlandse Vereniging Voor Reumatologie. Richtlijn Jicht. Utrecht; 2013. [Dutch Society for Rheumatology. Gout Guideline. Utrecht 2013.]

5. Richette P, Doherty M, Pascual E, Barskova V, Becce F, Castañeda-Sanabria J, et al. 2016 updated EULAR evidence-based recommendations for the management of gout. Ann Rheum Dis. 2017;76(1):29-42.

6. Khanna D, Khanna PP, Fitzgerald JD, Singh MK, Bae S, Neogi T, et al. 2012 American college of rheumatology guidelines for management of gout. part 2: Therapy and antiinflammatory prophylaxis of acute gouty arthritis. Arthritis Care Res. 2012;64(10):1447-61.

7. Janssens HJ, Janssen M, van de Lisdonk EH, van Riel PL, van Weel C. Use of oral prednisolone or naproxen for the treatment of gout arthritis: a double-blind, randomised equivalence trial. Lancet. 2008;371(9627):1854-60.

8. Wechalekar MD, Vinik O, Moi JHY, Sivera F, Van Echteld IAAM, van Durme C, et al. The efficacy and safety of treatments for acute gout: Results from a series of systematic literature reviews including cochrane reviews on intraarticular glucocorticoids, colchicine, nonsteroidal antiinflammatory drugs, and interleukin-1 inhibitors. J Rheumatol. 2014;92:15-25.

9. Keenan RT, O'Brien WR, Lee KH, Crittenden DB, Fisher MC, Goldfarb DS, et al. Prevalence of contraindications and prescription of pharmacologic therapies for gout. Am J Med. 2011;124(2):155-63.

10. Bardin T, Richette P. Impact of comorbidities on gout and hyperuricaemia: An update on prevalence and treatment options. BMC Med. 2017;15(123):1-10.

11. Pascart T, Lioté F. Gout: state of the art after a decade of developments. Rheumatology. 2019;58(1):27-44.

12. Cavalli G, Dinarello CA. Treating rheumatological diseases and co-morbidities with interleukin-1 blocking therapies. Rheumatology. 2015;54(12):2134-44.

13. So A, Dumusc A, Nasi S. The role of IL-1 in gout: from bench to bedside. Rheumatology. 2018;57:i12-9.

14. Petite SE. Effectiveness of Anakinra in Acute Gout: A Retrospective Review of Initial and Refractory Therapy. Am J Ther. 2017;24(5):e633-34.

15. McGonagle D, Tan AL, Shankaranarayana S, Madden J, Emery P, McDermott MF. Management of treatment resistant inflammation of acute on chronic tophaceous gout with anakinra. Ann Rheum Dis. 2007;66(12):1683-4.

16. Gratton SB, Scalapino KJ, Fye KH. Case of anakinra as a steroid-sparing agent for gout inflammation. Arthritis Care Res. 2009;61(9):1268-70.

17. Thueringer JT, Doll NK, Gertner E. Anakinra for the treatment of acute severe gout in critically ill patients. Semin Arthritis Rheum. 2015;45(1):81-5.

18. Chen K, Fields T, Mancuso CA, Bass AR, Vasanth L. Anakinra's Efficacy is Variable in Refractory Gout: Report 
of Ten Cases. Semin Arthritis Rheum. 2010;40(3):210-4.

19. Avram A, Duarte C, José Santos M, Papagoras C, Ritis K, Scarpioni R, et al. Identifying Patient Candidates for IL-1 Inhibition: Lessons From Real-World Cases. Jt Bone Spine 82. 2015; eS17-eS29.

20. Balasubramaniam G, Parker T, Turner D, Parker M, Scales J, Harnett P, et al. Feasibility randomised multicentre, double-blind, double-dummy controlled trial of anakinra, an interleukin-1 receptor antagonist versus intramuscular methylprednisolone for acute gout attacks in patients with chronic kidney disease (ASGARD): Protocol stu. BMJ Open. 2017;7(9):1-11.

21. Nocturne G, Ora J, Ea H-K, Lioté F. Influenza A H1N1 and anakinra exposure in a patient with gout. Jt Bone Spine. 2010;77(4):369-7

22. Van Wabeke J, Dhondt E, Peene I, Piette Y. Anakinra in resistant gout: a case report. Acta Clin Belg. $2016 ; 72(4): 293-5$.

23. Tran AP, Edelman J. Interleukin-1 inhibition by anakinra in refractory chronic tophaceous gout. Int J Rheum Dis. $2011 ; 14(3): 33-7$

24. Aouba A, Deshayes S, Frenzel L, Decottignies A, Pressiat C, Bienvenu B, et al. Efficacy of anakinra for various types of crystal-induced arthritis in complex hospitalized patients: a case series and review of the literature. Mediators Inflamm. 2015;2015:7.

25. Vitale A, Cantarini L, Rigante D, Bardelli M, Galeazzi M. Anakinra treatment in patients with gout and type 2 diabetes. Clin Rheumatol. 2015;34(5):981-4.

26. So A, De Smedt T, Revaz S, Tschopp J. A pilot study of IL-1 inhibition by anakinra in acute gout. Arthritis Res Ther. 2007;9(2):R28.

27. Ottaviani S, Moltó A, Ea H-K, Neveu S, Gill G, Brunier L, et al. Efficacy of anakinra in gouty arthritis: a retrospective study of 40 cases. Arthritis Res Ther. 2013;15(5):R123.

28. Ghosh P, Cho M, Rawat G, Simkin PA, Gardner GC. Treatment of acute gouty arthritis in complex hospitalized patients with anakinra. Arthritis Care Res. 2013;65(8):1381-4.

29. Singh D, Huston KK. IL-1 Inhibition With Anakinra in a Patient With Refractory Gout. J Clin Rheumatol. $2009 ; 15(7): 366$

30. Singh JA, Taylor WJ, Dalbeth N, Simon LS, Sundy J, Grainger R, et al. OMERACT endorsement of measures of outcome for studies of acute gout. J Rheumatol. 2014;41(3):569-73.

31. Willburger RE, Mysler E, Derbot J, Jung T, Thurston H, Kreiss A, et al. Lumiracoxib 400 mg once daily is comparable to indomethacin $50 \mathrm{mg}$ three times daily for the treatment of acute flares of gout. Rheumatology. 2007;46(7):1126-32.

32. Terkeltaub RA, Schumacher HR, Carter JD, Baraf HSB, Evans RR, Wang J, et al. Rilonacept in the treatment of acute gouty arthritis: a randomized, controlled clinical trial using indomethacin as the active comparator. Arthritis Res Ther. 2013;15:R25.

33. Fleming TR. Current issues in non-inferiority trials. Stat Med. 2008;27:317-32.

34. Schumacher Jr HR, Boice JA, Daikh DI, Mukhopadhyay S, Malmstrom K, Ng J, et al. Randomised double blind trial of etoricoxib and indometacin in treatment of acute gouty arthritis. BMJ. 2002;324(7352):1488-92.

35. Rubin BR, Burton R, Navarra S, Antigua J, Londono J, Pryhuber KG, et al. Efficacy and Safety Profile of 
Treatment with Etoricoxib 120 mg Once Daily Compared with Indomethacin 50 mg Three Times Daily in Acute Gout: A Randomized Controlled Trial. Arthritis Rheum. 2004;50(2):598-606.

36. So A, De Meulemeester M, Pikhlak A, Yücel AE, Richard D, Murphy V, et al. Canakinumab for the treatment of acute flares in difficult-to-treat gouty arthritis: Results of a multicenter, phase II, dose-ranging study. Arthritis Rheum. 2010 Jun 8;62(10):3064-76.

37. Rainer TH, Cheng CH, Janssens HJEM, Man CY, Tam LS, Choi YF, et al. Oral prednisolone in the treatment of acute gout: A pragmatic, multicenter, double-blind, randomized trial. Ann Intern Med. 2016;164(7):464-71.

38. Sterne JAC, White IR, Carlin JB, Spratt M, Royston P, Kenward MG, et al. Multiple imputation for missing data in epidemiological and clinical research: potential and pitfalls. BMJ. 2009 Jan;338:b2393.

39. Piaggio G, Elbourne DR, Pocock SJ, Evans SJW, Altman DG. Reporting of Noninferiority and Equivalence Randomized Trials: Extension of the CONSORT 2010 Statement. JAMA J Am Med Assoc. 2012;308(24):2594604.

40. Dönmez S, Pamuk ÖN. Chronic tophaceous gout. J Rheumatol. 2014;41(3):554-5.

41. Direz G, Noel N, Guyot C, Toupance O, Salmon J-H, Eschard J-P. Efficacy but side effects of anakinra therapy for chronic refractory gout in a renal transplant recipient with preterminal chronic renal failure. Jt Bone Spine. 2012;79(6):631.

42. Bartov JB, Ali Y. Successful Use of the Interleukin 1 Antagonist, Anakinra, in a Patient With Gout, Chronic Kidney Disease, and Aplastic Anemia. J Clin Rheumatol. 2013;19(8):454-6.

43. Funck-Brentano T, Salliot C, Leboime A, Zafrani L, Servais A, Larousserie F, et al. First observation of the efficacy of IL-1ra to treat tophaceous gout of the lumbar spine. Rheumatology. 2011;50(3):622-4.

44. Schett G, Schauer C, Hoffmann M, Herrmann M. Why does the gout attack stop? A roadmap for the immune pathogenesis of gout. RMD Open. 2015;1(Suppl 1):1-5.

45. Khanna PP, Gladue HS, Singh MK, FitzGerald JD, Bae S, Prakash S, et al. Treatment of acute gout: A systematic review. Semin Arthritis Rheum. 2014 Aug;44(1):31-8.

46. Fisher LD, Gent M, Büller HR. Active-control trials: How would a new agent compare with placebo? A method illustrated with clopidogrel, aspirin, and placebo. Am Heart J. 2001;141(1):26-32.

47. Song F, Altman DG, Glenny A-M, Deeks JJ. Validity of indirect comparison for estimating efficacy of competing interventions: empirical evidence from published meta-analyses. Bmj. 2003;326:472-472.

48. Schlesinger N, De Meulemeester M, Pikhlak A, Yücel AE, Richard D, Murphy V, et al. Canakinumab relieves symptoms of acute flares and improves health-related quality of life in patients with difficult-to-treat Gouty Arthritis by suppressing inflammation: results of a randomized, dose-ranging study. Arthritis Res Ther. 2011;13(2):R53.

49. Schlesinger N, Alten RE, Bardin T, Schumacher HR, Bloch M, Gimona A, et al. Canakinumab for acute gouty arthritis in patients with limited treatment options: Results from two randomised, multicentre, activecontrolled, double-blind trials and their initial extensions. Ann Rheum Dis. 2012;71(11):1839-48.

50. Loustau C, Rosine N, Forien M, Ottaviani S, Juge PA, Lioté F, et al. Effectiveness and safety of anakinra in gout patients with stage 4-5 chronic kidney disease or kidney transplantation: A multicentre, retrospective study. Jt Bone Spine. 2018;85(6):755-60. 


\section{SUPPLEMENTAL MATERIAL}

Supplemental material 1: Odds ratio (OR) on days $2-5$ in reaching $\geq 50 \%$ decrease in mean Numeric Rating Scale (NRS) pain scores.

\begin{tabular}{llll}
\hline Day & $\mathbf{O R}^{1}$ & $\mathbf{9 5 \%} \mathbf{C l}$ & $\boldsymbol{P}$ \\
\hline 2 & 1.41 & $0.53,3.73$ & 0.50 \\
3 & 2.56 & $1.03,6.37$ & $0.04^{*}$ \\
4 & 1.69 & $0.68,4.18$ & 0.26 \\
5 & 1.19 & $0.43,3.27$ & 0.74 \\
\hline
\end{tabular}

* significant difference $p<0.05 . \mathrm{Cl}$, confidence interval

${ }^{1}$ Anakinra treatment arm $(n=43)$ versus treatment as usual arm $(n=45)$ 


\section{Prognostic factors}

associated with early gout flare recurrence in patients initiating urate-lowering therapy during an acute gout flare

Carly A. Janssen, Martijn A.H. Oude Voshaar, Peter M. ten Klooster, Harald E. Vonkeman, Mart A.F.J. van de Laar 


\section{ABSTRACT}

Lowering serum urate levels below the threshold for crystal formation with uratelowering therapy (ULT) has been associated with a lower risk for gout flare reoccurrences. However, gout patients on ULT still commonly suffer from recurring gout flares. The purpose of this study was to explore prognostic factors associated with gout flare recurrence within the first 3 months, in gout patients starting ULT during an acute gout flare. Post-hoc analysis of trial data on acute gout patients randomized to either gout flare standard of care or anakinra treatment were used, including baseline demographic, laboratory, clinical, and patient-reported variables, as well as 3-month follow-up data on gout flare recurrences. Only patients starting ULT at baseline were included. Using variable selection based on clinical relevance, univariate, and multivariate binary logistic regression analyses were done to examine predictors of gout flare reoccurrence. A total of 75 patients were included in this study, of which 36 (48\%) experienced a gout flare $\leq 3$ months post baseline. The multivariate regression analysis revealed that CRP levels $>30$ mg/L (OR 9.47) and lack of prophylaxis when starting ULT (OR 11.56) were independently associated with gout flare recurrence. Similar results were found for the univariate regression analyses. Our results show that CRP levels $>30 \mathrm{mg} / \mathrm{L}$ and lack of prophylaxis when starting ULT were prognostic factors for early gout flare reoccurrence in patients starting ULT during an acute gout flare.

\section{Key Points.}

- Gout flare recurrences were common within the first 3 months after starting uratelowering therapy in gout patients.

- Intake of prophylaxis when starting ULT had a strong protective effect on gout flare recurrences.

- $\quad$ C-reactive protein level $>30 \mathrm{mg} / \mathrm{L}$ was an additional prognostic factor for early ( $\leq 3$ months) gout flare reoccurrence in patients starting ULT during an acute gout flare. 


\section{INTRODUCTION}

Lowering serum urate (SUA) levels below the threshold for monosodium urate crystal formation to the recommended SUA target of $<0.36 \mathrm{mmol} / \mathrm{L}$ with urate-lowering therapy (ULT) has been associated with reduced risk of gout flare recurrences and other adverse outcomes ${ }^{[1,2]}$. Therefore, most current gout management guidelines recommend to consider and discuss ULT with every patient, and to initiate ULT for patients with frequent flares, arthropathy, renal impairment, or tophi ${ }^{[2,3]}$. Various guidelines agree that ULT should be initiated early, possibly even upon presentation of an acute gout $\operatorname{attack}^{[2-4]}$.

However, research to date shows that even if ULT is instated, gout flare recurrences are relatively common, particularly in the period immediately following initiation ${ }^{[1,5,6]}$. Moreover, previous studies have shown that frequently recurrent gout (i.e., having $\geq 3$ flares per year) is associated with work impairment and decreased health-related quality of life ${ }^{[7,8]}$. While preventing recurrent gout flares is considered a priority for improving gout outcomes, not much is currently known about prognostic factors that place patients at increased risk for recurrent gout flares, especially in the period shortly after initiating ULT ${ }^{[6,9,10]}$. In fact, no previous studies have been performed in patients who initiate ULT during an acute gout flare ${ }^{[11-13]}$.

Therefore, in the current study, we investigated the prognostic value of various demographic, clinical or patient-reported factors for gout flare recurrence within the first 3 months following ULT initiation, in gout patients starting ULT during an acute gout flare.

\section{METHODS}

\section{Data source \& patient population}

For this post-hoc analysis, we used data from a multicenter, double-blind, double-dummy, randomized, non-inferiority clinical trial, in which the efficacy of anakinra versus standard of care for the treatment of acute gout flares was studied among crystal-proven gout patients recruited from seven rheumatology clinics in The Netherlands ${ }^{[14]}$. At baseline, eligible patients with an acute gout flare were randomized to either treatment with anakinra or standard of care, including colchicine, naproxen or prednisolon. In addition, the study protocol stipulated that patients should be initiated on ULT. All patients in the standard of care arm receiving treatment with naproxen or colchicine for their acute gout flare, continued this treatment up for 90 days as flare prophylaxis when initiating 
ULT. Patients randomized to the standard of care arm and receiving prednisolon, and patients who were randomized to the anakinra arm were provided with medication for their acute gout flare only and were not given prophylaxis with this treatment during initiation of ULT. This was because prophylaxis with corticosteroids or anakinra is currently not recommended in gout guidelines ${ }^{[2]}$. Patients were followed up for 3 months. More details regarding the study are given elsewhere ${ }^{[14]}$. This study was approved by a local independent ethics committee and by the institutional review board of each participating center, and was performed in accordance with the declaration of Helsinki and Good Clinical Practice guidelines. All patients provided written informed consent.

For the present study, we excluded patients from the original study who did not initiate ULT at baseline or were lost to follow-up.

\section{Reporting gout flares}

During the 3-month follow-up, patients were instructed to contact their treating rheumatologist or rheumatology nurse when they believed they were experiencing a new gout flare. Evaluator confirmed flares were subsequently documented in the electronic case-report form. Gout flare medication was provided to patients at the discretion of the treating rheumatologist. Beside the evaluator confirmed flares, patients themselves could also report gout flares in their electronic case report form.

\section{Statistical analysis}

For all included patients, baseline characteristics were summarized using mean and standard deviations, number and percentages, or the median and first and third quartile (Q1, Q3), depending on the distribution of the variables. For all statistical analyses, the statistical package SPSS version 22 was used for Windows.

To determine if an association exists between baseline characteristics and the occurrence of a gout flare within the first 3 months following initiation of ULT, a multivariate binary logistic regression model was developed. Potential predictors of flare recurrence were selected from the baseline data, based on their potential clinical relevance, including the levels of patient-reported pain (measured on a 5-point rating scale), body mass index (BMI), sex, levels of c-reactive protein (CRP) and serum urate (SUA), age, type of gout (intermittent or chronic gout), and receiving prophylaxis during ULT initiation. All potential variables were dichotomized using receiver operating characteristic (ROC) curves with the Youden Index to determine optimal cutoff points.

Preceding the dichotomization of variables and the regression analysis, missing values 
for the potential predictors (pain 16.0\%, BMI 1.3\%, CRP 6.7\%, SUA 8.0\%) were replaced by plausible values according to the Multiple Imputation by predictive mean matching method, based on the assumption that the data were missing at random. All baseline variables, as well as ULT type and occurrence of a flare within 3 months post baseline, were used as input for the imputation model ${ }^{[15]}$. Ten imputed datasets were generated.

Both a weighted univariate and multivariate binary logistic regression analysis was performed with all the potential predictors. The multivariate regression model was done using conditional backward elimination, with $p>0.10$ as the cutoff probability of stepwise removal of variables. As backward elimination on the ten imputed datasets might cause differences in selection of variables for the final multivariate model in each dataset, one analysis was performed in which the ten generated datasets with multiply imputed data were stacked. In that analysis, individual cases were weighted by $0.10(1 /$ number of datasets of imputation) to correct the standard errors for the artificial inflation of the sample size ${ }^{[16]}$. The goodness of fit for the model was tested using the Hosmer and Lemeshow test. The model's ability to discriminate between gout flare occurrence (yes/no) was examined by determining the area under the ROC curve, whereby an area between 0.70 and $<0.80$ indicated adequate discriminative ability, and between 0.80 and $<0.90$ excellent discriminative ability ${ }^{[17]}$. For the covariates remaining in the final model at the last step, odds ratios (OR) including corresponding $p$ values and $95 \%$ confidence intervals (95\% Cl) were calculated.

\section{RESULTS}

\section{Patient selection \& baseline characteristics}

Of the original 88 included patients, two patients never initiated ULT during the study, eight patients failed to initiate ULT at baseline, and three patients were lost to follow-up, leaving a total of 75 eligible patients who were included in the present analysis. Patient characteristics are provided in Table 1.

The majority of patients ( $n=71,94.7 \%$ ) initiated ULT with allopurinol, of which $55(77.5 \%)$ had a starting dose of $100 \mathrm{mg}, 13(18.3 \%)$ a starting dose of $300 \mathrm{mg}$, and one (1.4\%) and two (2.8\%) patients a starting dose of $50 \mathrm{mg}$ and $150 \mathrm{mg}$, respectively. Benzbromarone was given as the initial ULT in two patients $(2.7 \%)$ at a starting dose of $50 \mathrm{mg}$, while febuxostat was given to two patients $(2.7 \%)$ of which one patient initiated at a dose of $40 \mathrm{mg}$ and another patient at a dose of $80 \mathrm{mg}$. 
Of the total 75 patients included in this analysis, 34 (45.3\%) received treatment with anakinra at baseline, and 41 (55\%) with standard of care, of which 14 (18.7\%) with prednisolon, 16 (21.3\%) with colchicine, and 11 (14.7\%) with naproxen, according to the original study protocol.

Table 1: Baseline patient characteristics

\begin{tabular}{ll}
\hline Characteristic & Total group $(\mathbf{N}=\mathbf{7 5})$ \\
\hline Age, years, mean $\pm \mathrm{SD}$ & $61.0 \pm 12.5$ \\
Male sex, $\mathrm{n}(\%)$ & $73(97.3)$ \\
$\mathrm{BMI}, \mathrm{kg} / \mathrm{m}^{2}$, mean $\pm \mathrm{SD}(\mathrm{N}=74)$ & $29.5 \pm 3.9$ \\
$\mathrm{SUA}, \mathrm{mmol} / \mathrm{L}$, mean $\pm \mathrm{SD}(\mathrm{N}=69)$ & $0.50 \pm 0.11$ \\
$\mathrm{CRP}, \mathrm{mg} / \mathrm{L}$, median $(\mathrm{Q} 1, \mathrm{Q} 3)(\mathrm{N}=70)$ & $14.0(5.0,29.5)$ \\
Intermittent gout, $\mathrm{n}(\%)^{\mathrm{a}}$ & $59(78.7)$ \\
Painb, mean $\pm \mathrm{SD}(\mathrm{N}=63)$ & $3.5 \pm 0.80$ \\
Received prophylaxis, $\mathrm{n}(\%)$ & $27(36)$ \\
\hline
\end{tabular}

SD, standard deviation; BMI, body mass index; SUA, serum urate; CRP, C-reactive protein.

${ }^{a}$ compared to patients having chronic gout. ${ }^{b}$ measured on a 5 -point rating scale: $1=$ none, $2=$ mild, $3=$ moderate, $4=$ a lot, $5=$ extreme.

\section{Gout flare recurrence}

In total, 36 (48\%) patients experienced a gout flare, including both evaluator confirmed and patient-reported flares, within the first 3 months after ULT initiation, with a mean (SD) and median (Q1, Q3) time to the first reported gout flare event of 34.9 (31.8) days and $21.0(12.5,41.8)$ days, respectively. Overall, the mean (SD) number of flares per patient was $0.67(0.81)$ flares. Of the patients that experienced a gout flare, $24(66.7 \%)$ had one flare, 10 (27.8\%) patients had two flares, and two (5.6\%) patients had three gout flares. The mean (SD) number of flares among the patients having gout flares was 1.39 (0.60).

The highest proportion of patients experiencing a gout flare recurrence received treatment with anakinra $(n=23,63.9 \%)$, followed by prednisolon $(n=8,22.2 \%)$, naproxen $(n=3,8.3 \%)$, and colchicine $(n=2,5.6 \%)$. Interestingly, the first two groups did not receive prophylaxis when starting ULT, while the latter two groups were provided with prophylaxis.

\section{Predictors of flare recurrence}

For all the baseline predictors included in the multivariate regression model, the results of the univariate binary logistic regressions are presented in Table 2.

Results of the multivariate regression analysis showed that the two baseline covariates 
CRP level and prophylaxis, remained in the model and were independently predictive of gout flare recurrence $(p \leq 0.10)$ (Table 3 ). The odds for gout flare recurrence were 9.47 times higher for patients with CRP levels above $30 \mathrm{mg} / \mathrm{L}$ compared to patients with CRP levels at or below this value at baseline. In addition, intake of prophylaxis had a strong protective effect $(\mathrm{OR}, 0.09)$. The overall goodness to fit of the multivariate model was acceptable $\left(X^{2}(7)=2.44 ; p=0.296\right)$, and the area under the ROC curve was high, 0.78 , implying the model had adequate discriminative ability.

Table 2: Univariate binary logistic regression analysis of baseline covariates, associated with the occurrence of reported gout flare events $\leq 3$ months post baseline

\begin{tabular}{|c|c|c|c|c|c|}
\hline \multirow[t]{2}{*}{ Baseline variable } & \multicolumn{2}{|c|}{ Flare occurrence, $\mathrm{N}(\%)^{a}$} & \multicolumn{2}{|c|}{ Binary logistic regression } & \multirow[b]{2}{*}{$p$} \\
\hline & Yes & No & OR & 95\% Cl (lower, upper) & \\
\hline \multicolumn{6}{|l|}{ Pain ${ }^{b}$} \\
\hline$>3$ & $22(56.4)$ & $17(43.6)$ & 2.13 & $0.84,5.35$ & 0.11 \\
\hline$\leq 3$ & $14(38.9)$ & $22(61.1)$ & - & - & - \\
\hline \multicolumn{6}{|l|}{ BMI } \\
\hline$>27 \mathrm{~kg} / \mathrm{m}^{2}$ & $28(50.0)$ & $28(50.0)$ & 1.38 & $0.48,3.93$ & 0.55 \\
\hline$\leq 27 \mathrm{~kg} / \mathrm{m}^{2}$ & $8(42.1)$ & $11(57.9)$ & - & - & - \\
\hline \multicolumn{6}{|l|}{ CRP } \\
\hline$>30 \mathrm{mg} / \mathrm{L}$ & $15(78.9)$ & $4(21.1)$ & 5.90 & $1.72,20.2$ & 0.01 \\
\hline$\leq 30 \mathrm{mg} / \mathrm{L}$ & $22(38.6)$ & $35(61.4)$ & - & - & - \\
\hline \multicolumn{6}{|l|}{ SUA } \\
\hline$>0.39 \mathrm{mmol} / \mathrm{L}$ & $33(50.8)$ & $32(49.2)$ & 2.03 & $0.50,8.13$ & 0.32 \\
\hline$\leq 0.39 \mathrm{mmol} / \mathrm{L}$ & $3(30.0)$ & $7(70.0)$ & - & - & - \\
\hline \multicolumn{6}{|l|}{ Age } \\
\hline$>60$ years & $25(61.0)$ & $16(39.0)$ & 3.27 & $1.26,8.48$ & 0.02 \\
\hline$\leq 60$ years & $11(32.4)$ & $23(67.6)$ & - & - & - \\
\hline \multicolumn{6}{|l|}{ Type of gout } \\
\hline Chronic gout & $8(50.0)$ & $8(50.0)$ & 1.11 & $0.37,3.34$ & 0.86 \\
\hline Intermittent gout & $28(47.5)$ & $31(52.5)$ & - & - & - \\
\hline \multicolumn{6}{|l|}{ Baseline prophylaxis } \\
\hline No & $31(64.6)$ & $17(35.4)$ & 8.02 & $2.57,25.0$ & 0.00 \\
\hline Yes & $5(18.5)$ & $22(81.5)$ & - & - & - \\
\hline \multicolumn{6}{|l|}{ Sex } \\
\hline Female & $1(50.0)$ & $1(50.0)$ & 1.09 & $0.07,18.0$ & 0.95 \\
\hline Male & 35 (47.9) & $38(52.1)$ & - & - & - \\
\hline
\end{tabular}


Table 3: Multivariate regression model of baseline covariates, associated with the occurrence of reported gout flare events $\leq 3$ months post baseline.

\begin{tabular}{llll}
\hline Baseline variable & OR & $\mathbf{9 5 \%} \mathbf{C l}$ & $\boldsymbol{p}$ \\
\hline $\mathrm{CRP}>30 \mathrm{mg} / \mathrm{L}$ & 9.47 & $2.06-43.51$ & $<0.01$ \\
No prophylaxis & 11.56 & $2.96-45.11$ & $<0.00$ \\
\hline Nagelkerke $R^{2}=0.40 .0 R, 0$ dds ratio: & Cl.
\end{tabular}

\section{DISCUSSION}

Our study showed that approximately half of the patients (48\%) had at least one gout flare recurrence in the first 3 months following ULT initiation during an acute gout flare. Various studies that have previously reported on flare rates, show similar, or higher, percentages of gout patients having flares when on ULT, although differences in study designs and patient populations make comparison difficult $[1,6,11,12,18,19]$. Overall, our findings add to the already existing literature reporting on the unfavorable occurrence of gout flares when on ULT.

Furthermore, our results showed that receiving prophylaxis at the moment of ULT initiation strongly protected against gout flare reoccurrence, which further substantiates the importance of prophylaxis when initiating ULT, as is currently recommended by gout management guidelines ${ }^{[2]}$. The subpopulation of patients that received prophylaxis in our study were provided prophylaxis for 3 months with colchicine or the non-steroidal anti-inflammatory drug (NSAID) naproxen. This was because we were interested in determining the prognostic value of prophylaxis in preventing gout flares shortly after starting ULT since gout flares are common during this period ${ }^{[1]}$. We must note, however, that in contemporary gout guidelines prophylaxis with these agents is recommended for the first 6 months when starting ULT ${ }^{[2]}$. This is supported by data showing that gout flare prevention was more beneficial with intake of 6 months prophylaxis compared to 8 weeks, without the occurrence of more adverse reactions ${ }^{[9]}$. Future studies would need to evaluate the differences in gout flare risk, but also adverse reactions, between 3 months prophylactic treatment, as applied in this study, compared to the recommended 6 months. Moreover, prophylaxis with agents other than colchicine or NSAIDs may need consideration in the future as well, for example, with interleukin-1 (IL-1) inhibitors. Namely, it has been shown in previous studies that the IL-1 inhibitors canakinumab and rilonacept have prophylactic potency in preventing gout flares ${ }^{[19-23]}$. In the study by Schlesinger et al. 2011, prophylaxis with single doses of canakinumab $\geq 50 \mathrm{mg}$ or four 
4-weekly doses $(50 \mathrm{mg}, 50 \mathrm{mg}, 25 \mathrm{mg}, 25 \mathrm{mg}$ ) provided a greater relief in gout flare recurrences compared to daily colchicine $0.5 \mathrm{mg}$ given for 16 weeks in gout patients starting ULT with allopurinol ${ }^{[20]}$. In this study, less patients experienced at least one gout flare in the study period when treated with canakinumab (15-18.5\%) versus colchicine (44\%). The various studies done on rilonacept (once weekly subcutaneous injection of 80 or $160 \mathrm{mg}$ ) also revealed that it was more beneficial in reducing the frequency of gout flare recurrences compared to placebo when on ULT. Here, the proportion of patients experiencing at least one gout flare in 16 weeks ranged from 16 to $26 \%$ for rilonacept and 47 to $56 \%$ for placebo ${ }^{[19,21-23]}$. Prophylactic treatment with IL-1 inhibitors may in particular be of relevance in those gout patients who cannot be treated with colchicine or NSAIDs.

Moreover, CRP levels above $30 \mathrm{mg} / \mathrm{L}$ were independently predictive of gout flare recurrence. To our knowledge, limited research is currently available on the possible relationship between CRP levels and developing recurrent gout flares. In previous studies, increased systemic inflammation, including elevated markers as CRP, has been associated with elevated SUA levels and with cardiovascular diseases ${ }^{[24-28]}$. Therefore, the potential role of CRP in predicting gout flares, and the nature of this possible association, including such underlying factors (e.g., SUA level, presence of comorbidities), may need to be explored in future studies, particularly in other gout populations.

Previous long-term ( $\geq 1$ year) retrospective studies have shown an association between increased SUA levels and an increased risk for gout flare occurrence $[1,11,12,18,29]$. Contrarily, results of our study did not reveal such an association and SUA levels to be prognostic for the reoccurrence of a gout flare in the short-term ( $\leq 3$ months). Although the reason for this observation cannot be determined from our study, it might be attributed to the fact that the study population was rather homogenous, consisting primarily of patients having a baseline SUA level (mean $=0.50 \mathrm{mmol} / \mathrm{L}$ ) well above the recommended SUA target value of $0.36 \mathrm{mmol} / \mathrm{L}$. Notably, previous studies that have applied the SUA value of $0.36 \mathrm{mmol} / \mathrm{L}$ as the cutoff value for dichotomization in regression models found an association between SUA level and gout flares, whereas in the present study we used a SUA dichotomization value of $0.39 \mathrm{mmol} / \mathrm{L}$ and found no association ${ }^{[1,11,12,18]}$.

The various clinical and patient-related baseline characteristics considered as potential prognostic factors, as well as the use of data in patients with crystal-proven gout are strengths of this study. However, this study also has some limitations. First, potential predictors such as the presence of comorbidities, medication usage, and diet (e.g., 
purine-rich foods) were not available for this study, although these have been suggested to be relevant with regard to gout flare reoccurrence ${ }^{\text {[30-32] }}$. This includes the effect of different types of ULT on gout flare risk, which we were not able to include as a predictor in the model due to the variety of ULT and starting doses that were applied, leading to a small number of subjects receiving each ULT. Also, patients' actual adherence to their ULT medication during the course of the study was not measured. Moreover, our study population was limited to patients treated at rheumatology centers, and our results should therefore be confirmed in studies in different populations. Another limitation of this study is that the recently validated definition of gout flares for use in gout clinical research was not used in this study, but gout flares reported by patients and evaluators were used [33]. Finally, as we studied predictors of gout flare recurrence in patients initiating ULT during an acute gout episode, the generalizability of our results to gout patients not on ULT, and starting ULT prior to or following a gout flare, is limited.

In conclusion, we demonstrated that CRP levels $>30 \mathrm{mg} / \mathrm{L}$ and lack of prophylaxis were independent, prognostic factors for early ( $\leq 3$ months) gout flare reoccurrence in gout patients initiating ULT during an acute gout flare.

Acknowledgements

None. 


\section{REFERENCES}

1. Sarawate CA, Patel PA, Schumacher HR, Yang W, Brewer KK, Bakst AW. Serum Urate Levels and Gout Flares Analysis from managed care data. J Clin Rheumatol. 2006;12(2):61-5.

2. Richette P, Doherty M, Pascual E, Barskova V, Becce F, Castañeda-Sanabria J, et al. 2016 updated EULAR evidence-based recommendations for the management of gout. Ann Rheum Dis. 2017;76(1):29-42.

3. Hui M, Carr A, Cameron S, Davenport G, Doherty M, Forrester H, et al. The British Society For Rheumatology guideline for the management of gout. Rheumatology. 2017;56(7):e1-20.

4. Khanna D, Fitzgerald JD, Khanna PP, Bae S, Singh MK, Neogi T, et al. 2012 American college of rheumatology guidelines for management of gout. part 1: Systematic nonpharmacologic and pharmacologic therapeutic approaches to hyperuricemia. Arthritis Care Res. 2012;64(10):1431-46.

5. Doherty M, Jansen TL, Nuki G, Pascual E, Perez-Ruiz F, Punzi L, et al. Gout: why is this curable disease so seldom cured? Ann Rheum Dis. 2012;71(11):1765-70.

6. Becker MA, MacDonald PA, Hunt BJ, Lademacher C, Joseph-Ridge N. Determinants of the clinical outcomes of gout during the first year of urate-lowering therapy. Nucleosides, Nucleotides and Nucleic Acids. $2008 ; 27(6-7): 585-91$.

7. Khanna PP, Nuki G, Bardin T, Tausche AK, Forsythe A, Goren A, et al. Tophi and frequent gout flares are associated with impairments to quality of life, productivity, and increased healthcare resource use: Results from a cross-sectional survey. Health Qual Life Outcomes. 2012;10:1-11.

8. Edwards NL, Sundy JS, Forsythe A, Blume S, Pan F, Becker MA. Work productivity loss due to flares in patients with chronic gout refractory to conventional therapy. J Med Econ. 2011;14(1):10-5.

9. Wortmann RL, MacDonald PA, Hunt B, Jackson RL. Effect of Prophylaxis on Gout Flares After the Initiation of Urate-Lowering Therapy: Analysis of Data From Three Phase III Trials. Clin Ther. 2010;32(14):2386-97.

10. Dalbeth N, Choi HK, Terkeltaub R. Gout: A roadmap to approaches for improving global outcomes. Arthritis Rheumatol. 2017;69(1):22-34.

11. Rashid N, Levy GD, Wu Y-L, Zheng C, Koblick R, Cheetham TC. Patient and clinical characteristics associated with gout flares in an integrated healthcare system. Rheumatol Int. 2015;35(11):1799-807.

12. Halpern R, Fuldeore MJ, Mody RR, Patel PA, Mikuls TR. The effect of serum urate on gout flares and their associated costs: An administrative claims analysis. J Clin Rheumatol. 2009;15(1):3-7.

13. Wu E, Patel P, Mody R, Yu A, Cahill K, Tang J, et al. Frequency, risk, and cost of gout-related episodes among the elderly: does serum uric acid level matter? J Rheumatol. 2009;36(5):1032-40.

14. Janssen CA, Oude Voshaar MAH, Vonkeman HE, Jansen TLTA, Janssen M, Kok MR, et al. Anakinra for the treatment of acute gout flares: a randomized, double-blind, placebo-controlled, active-comparator, noninferiority trial. Rheumatology. 2019; [Epub ahead of print]. doi.org/10.1093/rheumatology/key402.

15. Sterne JAC, White IR, Carlin JB, Spratt M, Royston P, Kenward MG, et al. Multiple imputation for missing data in epidemiological and clinical research: potential and pitfalls. BMJ. 2009 Jan;338:b2393.

16. Wood AM, White IR, Royston P. How should variable selection be performed with multiply imputed data? Stat Med. 2008;27:3227-46. 
17. Hosmer DW, Lemeshow S. Applied Logistic Regression. 2nd ed. New York, NY: John Wiley \& Sons, Inc.; 2000.

18. Annemans L, Spaepen E, Gaskin M, Bonnemaire M, Malier V, Gilbert T, et al. Gout in the UK and Germany: prevalence, comorbidities and management in general practice 2000-2005. Ann Rheum Dis. 2008;67(7):960-6.

19. Sundy JS, Schumacher HR, Kivitz A, Weinstein SP, Wu R, King-Davis S, et al. Rilonacept for gout flare prevention in patients receiving uric acid-lowering therapy: Results of RESURGE, a phase III, international safety study. J Rheumatol. 2014;41(8):1703-11.

20. Schlesinger N, Mysler E, Lin H-Y, De Meulemeester M, Rovensky J, Arulmani U, et al. Canakinumab reduces the risk of acute gouty arthritis flares during initiation of allopurinol treatment: results of a double-blind, randomised study. Ann Rheum Dis. 2011;70(7):1264-71.

21. Mitha E, Ralph Schumacher H, Fouche L, Luo SF, Weinstein SP, Yancopoulos GD, et al. Rilonacept for gout flare prevention during initiation of uric acid-lowering therapy: Results from the PRESURGE-2 international, phase 3, randomized, placebo-controlled trial. Rheumatol (United Kingdom). 2013;52(7):1285-92.

22. Schumacher HR, Evans RR, Saag KG, Clower J, Jennings W, Weinstein SP, et al. Rilonacept (interleukin-1 trap) for prevention of gout flares during initiation of uric acid-lowering therapy: Results from a phase III randomized, double-blind, placebo-controlled, confirmatory efficacy study. Arthritis Care Res. 2012;64(10):1462-70

23. Schumacher HR, Sundy JS, Terkeltaub R, Knapp HR, Mellis SJ, Stahl N, et al. Rilonacept (interleukin-1 trap) in the prevention of acute gout flares during initiation of urate-lowering therapy: Results of a phase II randomized, double-blind, placebo-controlled trial. Arthritis Rheum. 2012;64(3):876-84.

24. Kang D, Park S, Lee I, Johnson R. Uric Acid-Induced C-Reactive Protein Expression: Implication on Cell Proliferation and Nitric Oxide Production of Human Vascular Cells. J Am Soc Nephrol. 2005;16(12):3553-62.

25. Grainger R, McLaughlin RJ, Harrison AA, Harper JL. Hyperuricaemia elevates circulating CCL2 levels and primes monocyte trafficking in subjects with inter-critical gout. Rheumatol (United Kingdom). 2012;52(6):1018-21

26. Keenan T, Blaha M, Nasir K, Silverman M, Tota-Maharaj R, Carvalho J, et al. Relation of Uric Acid to Serum Levels of High-Sensitivity C- Reactive Protein, Triglycerides, and High-Density Lipoprotein Cholesterol and to Hepatic Steatosis. Am J Cardiol. 2012;110(12):1787-92

27. Mason JC, Libby P. Cardiovascular disease in patients with chronic inflammation: Mechanisms underlying premature cardiovascular events in rheumatologic conditions. Eur Heart J. 2015;36(8):482-9.

28. Kaplan RC, Frishman WH. Systemic inflammation as a cardiovascular disease risk factor and as a potential target for drug therapy. Hear Dis. 2001;3(5):326-32.

29. Shoji A, Yamanaka H, Kamatani N. A Retrospective Study of the Relationship Between Serum Urate Level and Recurrent Attacks of Gouty Arthritis: Evidence for Reduction of Recurrent Gouty Arthritis With Antihyperuricemic Therapy. Arthritis Rheum. 2004;51(3):321-5.

30. Hunter D, York M, Chaisson C, Woods R, Niu J, Zhang Y. Recent diuretic use and the risk of recurrent gout attacks: the online case-crossover gout study. J Rheumatol. 2006;33(7):1341-5.

31. Primatesta P, Plana E, Rothenbacher D. Gout treatment and comorbidities: A retrospective cohort study in 
a large US managed care population. BMC Musculoskelet Disord. 2011;12:103.

32. Zhang Y, Chen C, Choi H, Chaisson C, Hunter D, Niu J, et al. Purine-rich foods intake and recurrent gout attacks. Ann Rheum Dis. 2012;71(9):1448-53.

33. Gaffo A, Dalbeth N, Saag K, Singh J, Rahn E, Mudano A, et al. Brief Report: Validation of a Definition of Flare in Patients With Established Gout. Arthritis Rheumatol. 2018;70(3):462-7. 


\section{Quality of care in gout: a clinical audit on treating to the target with urate lowering therapy in real-world gout patients}

Carly A. Janssen, Tim L.Th.A. Jansen, Martijn A.H. Oude Voshaar, Harald E. Vonkeman, Mart A.F.J. van de Laar 


\section{ABSTRACT}

The current paper aimed to describe the quality of care for gout patients by showing the clinical outcomes achieved in two patient cohorts in which differing targeted urate lowering therapy (ULT) treatment approaches were employed, both aiming to reach the European League Against Rheumatism recommended serum urate (SUA) targets. A retrospective medical chart review study was conducted. Data from the medical records of gout patients from two clinical centers in The Netherlands, both applying targeted ULT treatments (albeit using different approaches), were reviewed. Patients in cohort A were given a combination of xanthine oxidase inhibitors with uricosurics if treatment with allopurinol monotherapy failed to reach SUA target levels, whereas patients in cohort B were treated with sequential monotherapy. Data on patient characteristics and clinical outcomes were collected. A total of 177 patient dossiers were included: 99 from cohort A and 78 from cohort B. The great majority ( $n=146,82.5 \%$ ) of the patients in both cohorts had a current SUA level $<360 \mu \mathrm{mol} / \mathrm{L}$. In addition, more than half (n $=104,58.8 \%$ ) of the patients met the stringent SUA target level of $<300 \mu \mathrm{mol} / \mathrm{L}$. The largest reductions in mean SUA levels were observed for patients who were treated with combination therapy. This clinical audit of two cohorts of gout patients provides initialyet promising-results regarding the proportion of real-world gout patients in whom recommended SUA target levels can be achieved, and demonstrates the added value that a targeted treatment approach may have in reaching these goals. 


\section{INTRODUCTION}

Gout is one of the most common rheumatic diseases, with a particularly high prevalence in developed countries, where a growing percentage of the population is suffering from obesity and other gout-related comorbid conditions ${ }^{[1]}$. Various reports have shown the prevalence of gout to be increasing over time, which is frequently attributed to changing lifestyles, the increasing longevity of the population, and the accumulation of gout risk factors in older age ${ }^{[1-3]}$. Gout is a crystal-induced arthritis that may develop when serum uric acid levels surpass the saturation point for monosodium urate. Typically, gout initially presents as intermittent episodes of inflammation at the first metatarsophalangeal joint, recognized for causing moderate to severe pain, erythema, and joint swelling ${ }^{[4]}$. If left untreated, a chronic course may develop, characterized by persistent inflammation and visible urate deposits (referred to as tophi), potentially causing bone erosion, irreversible joint damage, and significant disability ${ }^{[5]}$.

The progression or recurrence of disease may be prevented with urate lowering therapy (ULT) that aims to reduce and maintain serum urate (SUA) levels below the saturation point for monosodium urate ${ }^{[6-8]}$. Although strategies for treating hyperuricemia have proven to be efficacious in clinical trials, the quality of care and management of gout in clinical practice are rarely reported and evidently remain suboptimal as gout patients are reportedly still often misdiagnosed and undertreated ${ }^{[9,10]}$. In 2006, the European League Against Rheumatism (EULAR) convened a task force to address these challenges by publishing management guidelines ${ }^{[6,11]}$. Recently, these guidelines have been updated to incorporate new developments in the understanding of gout pathophysiology and the availability of novel therapeutic options ${ }^{[12]}$.

Following recommendations for other rheumatic and non-rheumatic conditions, in which treating to a therapeutic target is becoming the standard of care, the 2006 and the updated guidelines for the management of gout recommend lowering and maintaining the SUA $<6 \mathrm{mg} / \mathrm{dL}(360 \mu \mathrm{mol} / \mathrm{L})$ for all patients on ULT ${ }^{[6,12]}$. For patients with severe gout, a target of $<5 \mathrm{mg} / \mathrm{dL}(300 \mu \mathrm{mol} / \mathrm{L})$ is recommended. However, evidence supporting the added value and feasibility of applying a treat-to-target (T2T) approach in normalizing SUA levels in gout patients remains largely absent and these recommendations are, therefore, currently based on expert opinion alone ${ }^{[13]}$.

In this paper, we describe the quality of care in gout by showing the clinical outcomes achieved in two Dutch patient cohorts in which different targeted treatment approaches were employed; however, both aim to reach the EULAR-recommended SUA targets. 


\section{METHODS}

\section{Study sites and patients}

A retrospective medical chart review study was conducted among patients suffering from gout, being treated at the rheumatology departments of two rheumatology clinics in The Netherlands, with both clinics using targeted ULT treatment approaches. At the first clinic, the medical records of patients with a clinical diagnosis of gout being followed up for their gout at the outpatient department rheumatology, while using ULT in the maintenance phase (3 months or longer), were included and are referred to as cohort A. At the time of inclusion, none of these patients had pre-terminal renal insufficiency $(<10 \mathrm{ml} / \mathrm{min})$ or were under treatment for gout flares with colchicine, prednisolone, or nonsteroidal anti-inflammatory drugs. At the second clinic, the medical records of all patients with a clinical diagnosis of gout were included and are referred to as cohort $B$. No exclusion criteria were employed. For both cohorts, patient monitoring visits, along with laboratory measurements, had been carried out to meet local standard of care procedures and patient needs.

In cohort A, a ULT-targeted treatment approach was employed allowing combination of xanthine oxidation inhibitors with uricosurics if treatment with allopurinol monotherapy failed to reach the SUA target of $<360 \mu \mathrm{mol} / \mathrm{L}$ and ideally $<300 \mu \mathrm{mol} / \mathrm{L}$ provided that the patient's kidney and liver functions, as well as medication tolerance posed no restrictions. In cohort B, a sequential monotherapy approach was followed that aimed for an SUA level <360 $\mathrm{mmol} / \mathrm{L}$. In both cohorts, therapy selection, dosages, and switches were made at the discretion of the attending rheumatologist based upon the clinical and biochemical status of the patient and patient experiences.

Data from the patient dossiers were gathered during the period of September to October 2016 and we aimed to retrieve data from approximately 100 consecutive gout patient dossiers from each clinic. Given the nature of the study, and in accordance with the relevant Dutch laws, no approval by the ethical review board was required.

\section{Data collection and analyses}

Data collected from the electronic patient dossiers included patient characteristics (i.e., age, sex, weight, and length), treatment followed, and treatment dosages. Blood measurements were collected, including serum creatinine levels and SUA levels. The latter consisted of two SUA measurements: the maximum SUA level, representing the highest SUA level ever measured in the past, and the current SUA level at the time of data retrieval. 
All analyses were performed using SPSS, version 22. Categorical variables were summarized using percentages and compared between groups using Chi-square statistics. Continuous variables were summarized using the mean and standard deviation and compared using Welch's or paired samples $t$ tests, as appropriate. A significance level of $\alpha=0.05$ was maintained for all analyses. No corrections were applied for multiple comparisons.

\section{RESULTS}

\section{Patients' characteristics}

In total, 177 patient dossiers were included in the study, of which 99 were from cohort A and 78 from cohort B. In cohort A, all patients and, in cohort B, 62 patients (79\%) had crystal-proven gout. No significant differences were observed between the patient groups for age, gender, or body mass index (BMI). In both cohorts, the majority of patients (>80\%) were male with a mean (SD) age of $67(12)$ and 68 (12) years for cohorts $A$ and $B$, respectively. Patients in cohort A had a mean (SD) BMI of 30 (5) and in cohort B 30 (6). The mean (SD) serum creatinine in cohort $A$ was 123 (58) $\mu \mathrm{mol} / \mathrm{L}$, which was significantly higher than that of cohort B, namely, 101 (37) $\mu \mathrm{mol} / \mathrm{L}$. The mean (SD) maximum SUA level was significantly higher in patients in cohort $A$ at 588 (115) $\mu \mathrm{mol} / \mathrm{L}$ than in cohort $B$ at 545 (74) $\mu \mathrm{mol} / \mathrm{L}$, while for the mean (SD) current SUA level, no significant difference was found between cohorts $A$ and $B$, at 282 (89) $\mu \mathrm{mol} / \mathrm{L}$ and 300 (77) $\mu \mathrm{mol} / \mathrm{L}$, respectively.

\section{Meeting the SUA targets}

At the time of the study, 146 out of the 177 patients ( $82.5 \%$ ) had a current SUA below the SUA target of $360 \mu \mathrm{mol} / \mathrm{L}$. More than half of the total sample ( $n=104,58.8 \%$ ) also had a current SUA below the more stringent target of $300 \mu \mathrm{mol} / \mathrm{L}$. In cohort B, $74.0 \%(n=57)$ of the patients had a current SUA below $360 \mu \mathrm{mol} / \mathrm{L}$ when being treated with allopurinol monotherapy at the time of the study. In cohort A, this was $45.5 \%$ ( $n=45)$. With the use of second-line ULT treatment options, a large proportion of the patients in each independent cohort were able to meet the $<360 \mu \mathrm{mol} / \mathrm{L}$ SUA target, with 80 patients $(80.8 \%)$ in cohort A showing a current SUA below the SUA target and 66 patients $(85.7 \%)$ in cohort B (Fig. 1). The stringent target of $<300 \mu \mathrm{mol} / \mathrm{L}$ was met by 44 patients $(57.1 \%)$ in cohort $B$ and in 60 patients $(60.6 \%)$ in cohort $A$. 


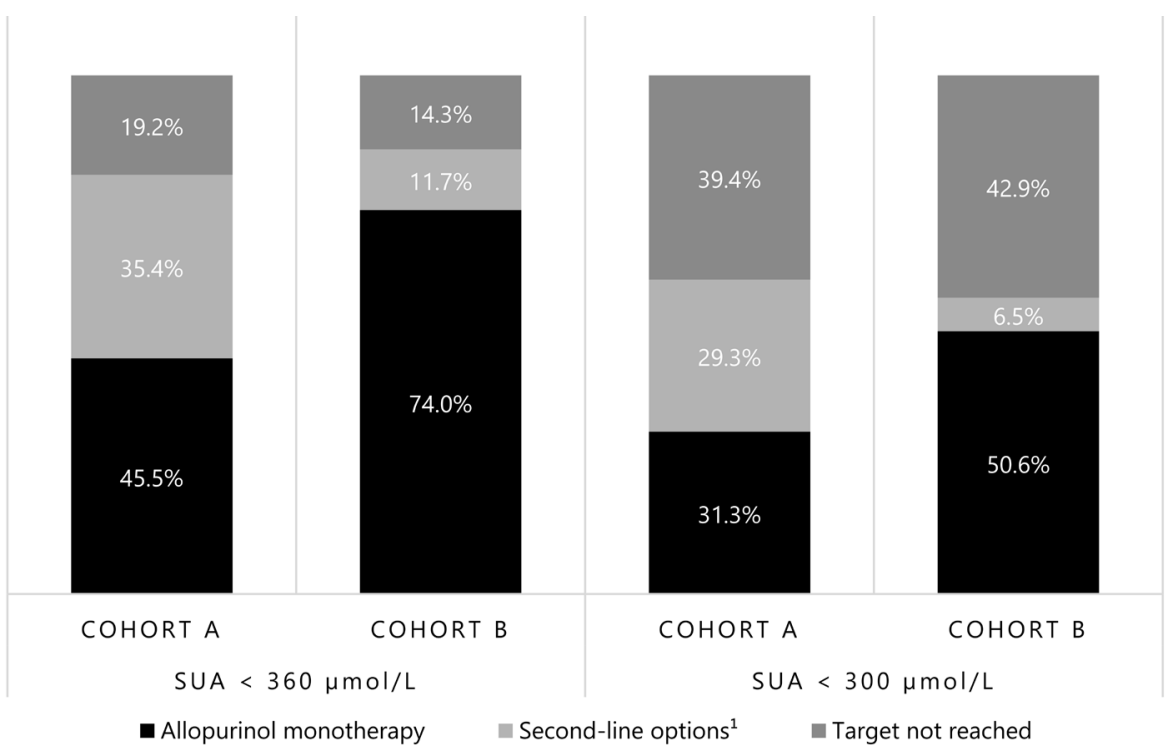

Fig. 1 Percentage (\%) of gout patients in each cohort meeting the EULAR-recommended serum urate (SUA) target levels of $<360$ and $<300 \mu \mathrm{mol} / \mathrm{L}$ at the time of the study with use of allopurinol monotherapy and second-line treatment options. Febuxostat monotherapy, benzbromarone monotherapy, and combination therapy are together defined as second-line treatment options. ${ }^{1}$ Combination therapy was only applied in cohort $\mathrm{A}$

\section{Descriptive results per ULT treatment option}

\section{Medication use}

Table 1 summarizes the ULT medication use at the time of the study, stratified by cohort. In general, patients were treated more aggressively in cohort A. Allopurinol, febuxostat, and benzbromarone monotherapy were all given at higher mean (SD) dosages (milligram) to cohort A [373 (169); 80 (15); 75 (29), respectively] than to cohort B [302 (116); 53 (21); $60(22)]$.

\section{$\underline{\text { SUA levels }}$}

The second part of Table 1 summarizes the mean (SD) SUA maximum and SUA current per ULT treatment group, along with the percentages of patients within each group achieving SUA target levels. The subpopulations of patients treated with second-line monotherapy (including both febuxostat and benzbromarone monotherapy) or combination therapy had a significantly higher mean (SD) maximum SUA compared with patients treated with allopurinol monotherapy at the time of the study, 591 (99) $\mu \mathrm{mol} / \mathrm{L}, 658$ (150) $\mu \mathrm{mol} / \mathrm{L}$, 
and 549 (82) $\mu \mathrm{mol} / \mathrm{L}$, respectively. Patients treated with combination therapies also achieved a substantially greater mean (SD) decrease in SUA levels compared to other patient treatment groups [432 (152) $\mu \mathrm{mol} / \mathrm{L}$ versus 294 (115) $\mu \mathrm{mol} / \mathrm{L}$ for second-line monotherapy and 248 (106) $\mathrm{\mu mol} / \mathrm{L}$ for allopurinol monotherapy].

Table 1: Total number ( $\mathrm{N}$ ) and percentage (\%) of gout patients in cohort $\mathrm{A}$ and cohort $\mathrm{B}$ receiving urate lowering therapy (ULT) grouped per ULT type, including the maximum and current mean serum urate (SUA) levels and SUA targets met within each ULT treatment group.

\begin{tabular}{|c|c|c|c|c|c|}
\hline Type of ULT treatment & N (\%a) & $\begin{array}{l}\text { Mean (SD) } \\
\text { SUA } \mu \mathrm{mol} / \mathrm{L} \\
\text { maximum }\end{array}$ & $\begin{array}{l}\text { Mean (SD) } \\
\text { SUA } \mu \mathrm{mol} / \mathrm{L} \\
\text { current }\end{array}$ & $\begin{array}{l}\text { SUA }<360 \\
\mu \mathrm{mol} / \mathrm{L}, \\
\mathrm{N}(\%)\end{array}$ & $\begin{array}{l}\text { SUA }<300 \\
\mu \mathrm{mol} / \mathrm{L}, \\
\mathrm{N}(\%)\end{array}$ \\
\hline \multicolumn{6}{|l|}{ Cohort A } \\
\hline Allopurinol monotherapy & $58(58.6)$ & $558(94)$ & $300(86)$ & $45(77.6)$ & $31(53.4)$ \\
\hline Febuxostat monotherapy & $16(16.2)$ & 598 (99) & $277(85)$ & $14(87.5)$ & $11(68.8)$ \\
\hline Benzbromarone monotherapy & $4(4.0)$ & $608(131)$ & $330(65)$ & $2(50.0)$ & $1(25.0)$ \\
\hline $\begin{array}{l}\text { Allopurinol/Benzbromarone } \\
\text { combination therapy }\end{array}$ & $15(15.2)$ & $612(120)$ & $213(82)$ & $14(93.3)$ & $13(86.7)$ \\
\hline $\begin{array}{l}\text { Febuxostat/Benzbromarone } \\
\text { combination therapy }\end{array}$ & $6(6.1)$ & $793(163)$ & $255(91)$ & $5(83.3)$ & $4(66.7)$ \\
\hline \multicolumn{6}{|l|}{ Cohort B } \\
\hline Allopurinol monotherapy & $66(84.6)$ & $540(70)$ & $296(70)$ & $57(86.4)$ & $39(59.1)$ \\
\hline Febuxostat monotherapy & $6(7.7)$ & $592(111)$ & $380(90)$ & $4(66.7)$ & $1(16.7)$ \\
\hline Benzbromarone monotherapy & $5(6.4)$ & $554(81)$ & $234(58)$ & $5(100.0)$ & $4(80.0)$ \\
\hline No ULT & $1(1.3)$ & NA & NA & NA & NA \\
\hline
\end{tabular}

Total sample size of cohort $A=99$ patients and cohort $B=78$ patients

ULT: urate lowering therapy; NA: not applicable; SD: standard deviation; SUA: serum urate

a Sum of percentages within each cohort is equal to 100 .

${ }^{\mathrm{b}}$ Combination therapy was only given in cohort $\mathrm{A}$.

\section{DISCUSSION}

In this paper, we report the result of a clinical audit on the quality of care in gout using a retrospective chart review study in two independent cohorts in which gout patients were treated according to different ULT approaches, both aiming to achieve SUA target levels as recommended in the updated EULAR gout guidelines. The results presented here illustrate the SUA levels and clinical outcomes that may be reached in everyday clinical practice using currently available ULT and a targeted treatment approach. An analysis of our quality of care (the combination of prescribed treatment, instructing, and monitoring of gout patients) is of importance to get a sense of where opportunities may 
lie for further improvement.

Based on our data, we postulate that achieving EULAR-recommended SUA levels seems to be a realistic goal in clinical practice for gout patients, using currently available ULT, patient education, and monitoring of SUA-targeted treatment. The great majority $(82.5 \%)$ of the gout patients in the studied cohorts had a current SUA $<360 \mu \mathrm{mol} / \mathrm{L}$. This is in contrast to other studies in which much lower proportions of patients were able to meet SUA levels $<360 \mu \mathrm{mol} / \mathrm{L}$, ranging from 21 to $77 \%{ }^{[14-19]}$. In those studies, failure to reach SUA targets was seen as multifactorial, but mainly attributed to inadequate dose titration of ULT according to SUA measurements, as well as to infrequent patient monitoring and treatment incompliance. In our study, patient monitoring visits had been made according to daily routine practice and patient needs, in principle, allowing for ULT dosages and treatments to be adjusted according to SUA measurements, which might explain the higher percentages of patients meeting SUA targets in our study. Since both data on the frequency of patient monitoring visits and patient follow-up data were beyond the scope of this clinical audit, the extent to which dose and medication adjustments were actually steered in response to patients' current SUA levels could not be assessed. Moreover, between the rather similar cohorts, significant variation existed among the percentages of patients treated with allopurinol monotherapy and having a current SUA below $360 \mu \mathrm{mol} / \mathrm{L}$. This could have occurred, because a more stringent target of $<300 \mu \mathrm{mol} / \mathrm{L}$ was striven for in some patients in cohort A. However, physiciandependent or unobserved patient-related factors might also have contributed to changes in treatment options or dosage adjustments. Nonetheless, our study shows that most patients can achieve SUA target levels if medication is titrated to reach predefined SUA targets, providing a proof-of-concept of the feasibility of a T2T approach in gout.

In a T2T approach, patients typically follow a therapeutic process in which failing to respond to a treatment option leads to switching to other, perhaps more costly, treatment options to achieve prespecified SUA target values. In the current study, 31 gout patients (17.5\%) failed to have a current SUA below $360 \mu \mathrm{mol} / \mathrm{L}$ of whom eight patients (4.5\%) were already using the second-line options of monotherapy or combination therapy. Therefore, these patients could benefit from alternative therapy choices. One such treatment for patients with severe debilitating, chronic tophaceous gout, who have exhausted other treatment options, is pegloticase. Other therapies, such as the uricosuric lesinurad, might also become available for this patient group in the near future. The added value for the quality of care of gout patients of novel treatment options should be assessed in future real-life studies. 
There are a few limitations to this study. First, only gout patients undergoing follow-up at rheumatology departments were included in this study, probably leading to a sample of patients with severe or complex gout. Since in The Netherlands, the majority of gout patients are treated by their general practitioner, future studies in primary care settings seem warranted. Second, data relating to patient-reported outcomes, other gout outcomes (e.g. number of flares), treatment costs, and drug safety were not available for this study. Although previous studies have demonstrated considerable correlation between SUA and many of these, future prospective and clinical studies should include such outcome domains to better characterize potential differences between treatment strategies ${ }^{[5,19,20]}$. Strengths of the current study are the use of real-life data and the employment of few exclusion criteria, therefore, reflecting the experience of gout patients seen in daily clinical practice.

In summary, the findings of this study provide an initial proof-of-concept of the favorable outcomes in terms of the percentage of real-world gout patients for whom recommended SUA target levels can be achieved when using ULT, and the added value a targeted treatment approach may have in reaching these goals. To evaluate potential differences between treatment strategies regarding efficacy, safety, costs, patientreported outcomes, and the impact on society, prospective, pragmatic studies are needed to further investigate the T2T principle of SUA levels in gout patients.

\section{Acknowledgements}

None. 


\section{REFERENCES}

1. Kuo C-F, Grainge MJ, Zhang W, Doherty M. Global epidemiology of gout: prevalence, incidence and risk factors. Nat Rev Rheumatol. 2015;11(11):649-62.

2. Wallace KL, Riedel AA, Joseph-Ridge N, Wortmann R. Increasing prevalence of gout and hyperuricemia over 10 years among older adults in a managed care population. J Rheumatol. 2004;31(8):1582-7.

3. Bardin T, Bouée S, Clerson P, Chalès G, Flipo R-M, Lioté F, et al. Prevalence of Gout in the Adult Population of France. Arthritis Care Res. 2016;68(2):261-6.

4. Jansen TL. Chapter 41 Clinical presentation of gout. In: Doherty M, Hunter DJ, Bijlsma H, Arden N, Dalbeth N, editors. Oxford Textbook of Osteoarthritis and Crystal Arthropathy. 3rd editio. New York: Oxford University Press; 2016.

5. Becker MA, Schumacher HR, Benjamin KL, Gorevic P, Greenwald M, Fessel J, et al. Quality of life and disability in patients with treatment-failure gout. J Rheumatol. 2009;36(5):1041-8.

6. Zhang W, Doherty M, Bardin T, Pascual E, Barskova V, Conaghan P, et al. EULAR evidence based recommendations for gout. Part II: Management. Report of a task force of the EULAR Standing Committee for International Clinical Studies Including Therapeutics (ESCISIT). Ann Rheum Dis. 2006;65(10):1312-24.

7. Perez-Ruiz F. Treating to target: A strategy to cure gout. Rheumatology. 2009;48(suppl. 2):ii9-14.

8. Khanna D, Khanna PP, Fitzgerald JD, Singh MK, Bae S, Neogi T, et al. 2012 American college of rheumatology guidelines for management of gout. part 2: Therapy and antiinflammatory prophylaxis of acute gouty arthritis. Arthritis Care Res. 2012;64(10):1447-61.

9. Doherty M, Jansen TL, Nuki G, Pascual E, Perez-Ruiz F, Punzi L, et al. Gout: why is this curable disease so seldom cured? Ann Rheum Dis. 2012;71(11):1765-70.

10. Kuo C-F, Grainge MJ, Mallen C, Zhang W, Doherty M. Rising burden of gout in the UK but continuing suboptimal management: a nationwide population study. Ann Rheum Dis. 2015;74(4):661-7.

11. Zhang W, Doherty M, Pascual E, Bardin T, Barskova V, Conaghan P, et al. EULAR evidence based recommendations for gout. Part I: Diagnosis. Report of a task force of the Standing Committee for International Clinical Studies Including Therapeutics (ESCISIT). Ann Rheum Dis. 2006;65(10):1301-11.

12. Richette P, Doherty M, Pascual E, Barskova V, Becce F, Castañeda-Sanabria J, et al. 2016 updated EULAR evidence-based recommendations for the management of gout. Ann Rheum Dis. 2017;76(1):29-42.

13. Kiltz U, Smolen J, Bardin T, Cohen Solal A, Dalbeth N, Doherty M, et al. Treat-to-target (T2T) recommendations for gout. Ann Rheum Dis. 2017;76(4):632-8.

14. Cottrell E, Crabtree V, Edwards JJ, Roddy E. Improvement in the management of gout is vital and overdue: an audit from a UK primary care medical practice. BMC Fam Pract. 2013;14(1):170.

15. Arroll B, Bennett M, Dalbeth N, Hettiarachchi D, Cribben B, Shelling G. More allopurinol is needed to get gout patients < $0.36 \mathrm{mmol} / \mathrm{L}$ : a gout audit in the form of a before-after trial. J Prim Health Care. 2009;1(4):315-8.

16. Wall GC, Koenigsfeld CF, Hegge KA, Bottenberg MM. Adherence to treatment guidelines in two primary care populations with gout. Rheumatol Int. 2010;30(6):749-53.

17. Sarawate CA, Patel PA, Schumacher HR, Yang W, Brewer KK, Bakst AW. Serum Urate Levels and Gout Flares 
Analysis from managed care data. J Clin Rheumatol. 2006;12(2):61-5.

18. Roddy E, Zhang W, Doherty M. Concordance of the management of chronic gout in a UK primary-care population with the EULAR gout recommendations. Ann Rheum Dis. 2007;66(10):1311-5.

19. Khanna P, Khanna D, Storgard C, Baumgartner S, Morlock R. A world of hurt: failure to achieve treatment goals in patients with gout requires a paradigm shift. Postgrad Med. 2016;128(1):34-40.

20. Shiozawa A, Buysman EK, Korrer S. Serum uric acid levels and the risk of flares among gout patients in a US managed care setting. Curr Med Res Opin. 2017;33(1):117-24. 


\section{A retrospective analysis of medication prescription records for determining the levels of compliance and persistence to urate-lowering therapy for the treatment of gout and hyperuricemia in The Netherlands}

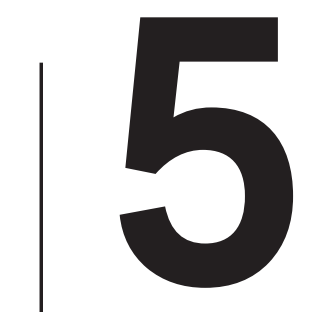

Carly A. Janssen, Martijn A.H. Oude Voshaar, Harald E. Vonkeman, Marieke Krol, Mart A.F.J. van de Laar 


\section{ABSTRACT}

Urate-lowering therapy (ULT) is a recommended life-long treatment for gout patients. However, despite these recommendations, recurrent gout attacks are commonly observed in clinical practice. The purpose of this study was to assess the levels of compliance and persistence to ULT in The Netherlands, in order to reflect on the current gout care delivered by health professionals. Anonymous prescription records were obtained from IQVIA's Dutch retrospective longitudinal prescription database, containing ULT dispensing data for allopurinol, febuxostat, and benzbromarone from November 2013 to July 2017. Compliance to ULT was determined by calculating the proportion of days covered (PDC) over 12 months. Persistence over 12 months was evaluated by determining the time to discontinuation, without surpassing a refill gap of $>30$ days. Association of PDC and persistence with age, gender, and first prescriber were examined using beta regression- and cox-regression models, respectively. There were 45,654 patients who met the inclusion criteria. Overall, $51.7 \%$ of the patients had a ULT coverage of $\geq 80 \%$ of the days in 1 year (PDC $\geq 0.80$ ), and $42.7 \%$ of the patients were still persistent after 1 year. Men, older patients, and patients whose first prescriber was a rheumatologist were more persistent and had a higher PDC. Our results show that medication adherence to ULT after 1 year is suboptimal, considering that current guidelines recommend ULT as a life-long treatment. Future studies addressing the reasons for treatment cessation and improving treatment adherence seem warranted. 


\section{INTRODUCTION}

Gout, which has become increasingly prevalent over the last decades, is currently one of the most common forms of inflammatory arthritis ${ }^{[1,2]}$. Acute gout flares result from prolonged hyperuricemia, which may lead to the formation and deposition of monosodium urate crystals in and around the joints, triggering an inflammatory response. Gout is associated with pain, swelling, tenderness, and erythema and may lead to physical disability and reduced quality of life ${ }^{[3,4]}$.

One of the major goals of gout management is lowering and managing serum urate levels by using urate-lowering therapy (ULT) to prevent gout attacks. Current guidelines recommend initiating ULT in patients with recurring gout flares and tophi, and to consider initiating ULT in all patients upon definite diagnosis of gout ${ }^{[5]}$. However, despite these recommendations and the availability of effective ULT, recurrent attacks of gouty arthritis are commonly observed in clinical practice ${ }^{[6]}$. These may result from both the lack of ULT initiation in gout patients by health professionals, but also poor medication adherence (or compliance) by patients ${ }^{[7-9]}$.

A recent systematic review and meta-analysis by Scheepers et al. 2017 shows a large variation in adherence to ULT across studies, with 12-month adherence rates ranging from 3 to $78 \%$, and non-persistence rates ranging from 54 to $87 \%$ after 1 year, in prescription and claims-based studies ${ }^{[10]}$. This could partly be explained by the fact that many of these studies were performed using data collected in health care administrative databases that apply to specific subpopulations of gout patients, for example, databases restricted to specific insurance types, or healthcare settings. Moreover, their results show that only three previous studies described adherence to ULT in European gout patients.

We set out to gain insight into the dispensing patterns, as well as the level of compliance and persistence to commonly prescribed ULT drugs for the treatment of hyperuricemia and gout, in a nationally representative study in the Netherlands.

\section{METHODS}

\section{Data source}

Data for this nationally representative, retrospective, prescription-based study were obtained from IQVIA's Real-World Data Longitudinal Prescription database (LRX, 
Amsterdam, The Netherlands). The database contains anonymous patient prescription records, including patient (e.g., age, gender), dispensing (e.g., pharmacy, prescription date), medication (e.g., name, dose, strength, therapy duration), and prescriber information. For this study, dispensed ULT prescriptions of allopurinol, febuxostat, and benzbromarone prescribed by rheumatologists, general practitioners, and internists were selected. In total, the database provides a coverage of approximately $75 \%$ of all prescriptions dispensed in The Netherlands, represented by both retail pharmacies and dispensing general practitioners. Data was available from the time period of 1 November 2013 up till 31 July 2017.

\section{Measuring compliance and persistence}

Reporting standards for retrospective database studies on measuring compliance and persistence were followed in describing the study results ${ }^{[11]}$. To identify patients newly starting on ULT, patients were selected who did not receive any ULT prescription (allopurinol, febuxostat, or benzbromarone) in the 6 months prior to their first ULT dispensing in the defined study enrollment period, which was between 1 May 2014 and 31 July 2016. Since ULT is also prescribed for conditions other than gout, and the diagnosis of patients was unknown, patients $<18$ years old at the time of first ULT dispensing were excluded. Similarly, patients whose baseline prescription was prescribed by a professional other than a general practitioner or rheumatologist, were excluded. However, follow-up prescriptions by internists were included for calculating compliance and persistence. Prescriptions with a dispensed duration of zero days were excluded.

Compliance to ULT was defined as the proportion of days covered (PDC) by medication over a period of 12 consecutive months, correcting for any overlap in prescriptions ${ }^{[11]}$. Patients were not required to be continuously using ULT (persistent). For comparability with previous studies, the PDC was dichotomized, and patients with PDC $\geq 0.80$ were considered compliant ${ }^{[10]}$. Persistence refers to the extent to which patients continue their medication over 12 months, without exceeding a refill gap of 30 days. The time to discontinuation was determined in days.

For the analyses of compliance and persistence, we made three assumptions; (1) intake of the medication by patients was done in accordance with the issued prescription, in terms of duration and frequency; (2) patients were initiated on lifelong ULT for the treatment of gout and hyperuricemia; and (3) dispensed prescriptions were considered to be taken for the first time on the same day of dispensing, except when a patient still had medication available from the previous prescription; in those cases medication intake started the day after the medication of the previous prescription was exhausted. 
For calculating compliance and persistence, no distinction was made in type of ULT received.

\section{Statistical analysis}

Means and standard deviations, or medians and first and third quartiles were used to summarize general characteristics of the patient population, dispensing patterns, as well as compliance and persistence levels after 1 year. Univariable and multivariable regression analyses were performed to identify factors associated with PDC and time to treatment cessation. Since the PDC variable is naturally bounded between 0 and 1 , and had a "U-shaped" distribution with relatively high numbers of patients at the extremes (i.e., $0.10 \geq P D C \geq 0.90$ ), we used beta regression analysis, with a logit link function. Overall goodness of fit was examined using the pseudo $R$-square statistic, and statistical significance of the coefficient estimates were tested using partial Wald statistics. This was accomplished by using the betareg $R$ package ${ }^{[12]}$. Factors associated with time to treatment cessation were studied using cox-regression analysis using IBM SPSS Statistics version 23. For testing the cox proportional hazard assumption, log minus log graphs were visually inspected.

\section{RESULTS}

\section{Patient characteristics}

ULT prescriptions of 130,232 patients were dispensed by rheumatologists, general practitioners, or an internist between November 2013 and July 2017. Of these, 45,654 patients met the selection criteria, of which the majority were male ( $n=34,761,76.1 \%$ ). The mean (SD) age was 65.7 (14.1) years.

\section{Dispensing patterns}

The total number of ULT prescriptions dispensed was 377,309 with the number of ULT prescriptions per patient ranging from 1 to 297, and a median (first, third quartile) of 5 $(3,7)$. The most common initial ULT prescription dispensed during the study enrollment period was allopurinol $(n=44,068,96.5 \%)$, followed by benzbromarone $(n=1386,3.0 \%)$ and febuxostat $(n=200,0.4 \%$ ). Most patients were issued their first ULT prescription by general practitioners $(n=37,917,83.1 \%$ ). The median (first, third quartile) ULT treatment duration dispensed was $30(15,60)$ for rheumatologists and $30(15,90)$ days for general practitioners, respectively. Table 1 provides an overview of the initial type of ULT and dosages dispensed by both prescribers. 
Table 1: Initial ULT dispensed among general practitioners and rheumatologists

\begin{tabular}{lll}
\hline Type of ULT & General practitioner, \% (N) & Rheumatologist, $\%(\mathbf{N})^{\mathbf{b}}$ \\
\hline Allopurinol $100 \mathrm{mg}$ & $75.9(28,773)$ & $72.1(5,576)$ \\
Allopurinol $200 \mathrm{mg}$ & $1.3(476)$ & $0.4(31)$ \\
Allopurinol $300 \mathrm{mg}$ & $20.1(7,630)$ & $20.4(1,582)$ \\
Febuxostat $80 \mathrm{mg}$ & $0.2(83)$ & $1.5(113)$ \\
Febuxostat $120 \mathrm{mg}$ & $0.0(4)$ & $0.0(0)$ \\
Benzbromarone 100mg & $2.5(951)$ & $5.6(435)$ \\
\hline
\end{tabular}

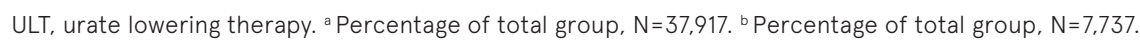

\section{Compliance}

PDC values had a median of 0.82 and first and third quartiles of 0.33 and 0.99 , respectively. In total, $51.7 \%(n=23,602)$ patients were categorized as compliant.

Gender, age, and first prescriber were found to be associated with PDC, according to the Wald statistics (Table 2). In the multivariable beta regression model, the predicted value for PDC was 53\%, when all covariates were set to zero. Ceteris paribus the predicted PDC value increased by $8 \%$ if a rheumatologist was the first prescriber instead of a general practitioner, and by $~ 3 \%$ for each increasing incremental decade of the patient's age. The predicted value for PDC decreased by $3 \%$ for female patients. The pseudo $R$ square for the multivariable model was 0.024 .

Table 2: Variables associated with PDC

\begin{tabular}{|c|c|c|c|c|c|c|}
\hline & \multicolumn{3}{|c|}{ Univariable analyses } & \multicolumn{3}{|c|}{ Multivariable analyses $^{a}$} \\
\hline & Gender & Age & Prescriber & Gender & Age & Prescriber \\
\hline Intercept & 0.957 & 0.252 & 0.890 & 0.139 & 0.139 & 0.139 \\
\hline$\beta$-coefficient & $-0.044^{\star}$ & $0.011^{\star *}$ & $0.336^{\star *}$ & $-0.127^{\star \star}$ & $0.012^{\star \star}$ & $0.348 * \star$ \\
\hline SE & 0.014 & $<0.000$ & 0.015 & 0.014 & $<0.000$ & 0.015 \\
\hline Pseudo $R^{2}$ & $<0.000$ & 0.011 & 0.011 & 0.024 & 0.024 & 0.024 \\
\hline
\end{tabular}

SE, standard error; PDC, proportion of days covered. ${ }^{a}$ Model: $\mathrm{PDC} \sim$ gender + age + prescriber. ${ }^{\star} P<0.01 ;{ }^{\star \star} P$ $<0.001$

\section{Persistence}

The median (first, third quartile) time to discontinuation of ULT was $248(83,420)$ days for the entire population, and after 1 year $42.7 \%(N=19,509)$ of the patients were still persistent. In general, major drops in persistence were seen after 30 ( $n=3827$ ) and 90 
( $n=4093$ ) days of treatment, which are common durations of prescribed ULT medications. Noticeably, for $50.2 \%(n=1922)$ and $38.9 \%(n=1593)$ of the patients stopping at day 30 and 90 respectively, only one ULT prescription was dispensed in 1 year time.

Visual inspection of the log-minus-log hazard function plots for age, gender, and first prescriber supported that the assumption of proportional hazards was met. When applying multivariate cox-regression for gender, age, and initial prescriber of ULT, hazard ratios (HR) differed significantly between groups (Table 3, Fig. 1). Males had a 10.3\% (HR $0.897,95 \% \mathrm{Cl} 0.87-0.92$ ) lower probability of treatment cessation at any point in time, compared to women. Also, older patients had a statistically significant lower probability of stopping treatment at any point in time compared to younger patients. Finally, the HR for patients initially treated by a rheumatologists compared to a general practitioner was 0.788 (95\% Cl, 0.76-0.82), suggesting the probability of discontinuing medication is $21.2 \%$ less likely at any point in time for patients initially treated by a rheumatologist.

Table 3: Multivariate cox-regression for persistence

\begin{tabular}{|c|c|c|c|c|}
\hline & HR & $95 \% \mathrm{Cl}$ & $\begin{array}{l}\text { Time to discontinuation in } \\
\text { days, median (Q1, Q3) }\end{array}$ & $\begin{array}{l}\text { Persistent at } \\
12 \text { months (\%) }\end{array}$ \\
\hline \multicolumn{5}{|l|}{ Gender } \\
\hline Female $^{a}$ & - & - & $236(60,401)$ & 41.9 \\
\hline Male & $0.897^{\star}$ & $0.87,0.92$ & $257(90,420)$ & 43.0 \\
\hline \multicolumn{5}{|l|}{ Age group } \\
\hline $18-60^{a}$ & - & - & $180(60,404)$ & 35.6 \\
\hline $61-73$ & $0.713^{\star}$ & $0.69,0.73$ & $333(90,438)$ & 47.5 \\
\hline$\geq 74$ & 0.750 * & $0.72,0.77$ & $286(85,404)$ & 45.0 \\
\hline \multicolumn{5}{|l|}{ Prescriber } \\
\hline General practitionera & - & - & $225(72,408)$ & 41.5 \\
\hline Rheumatologist & $0.788 *$ & $0.76,0.82$ & $359(90,450)$ & 48.9 \\
\hline
\end{tabular}

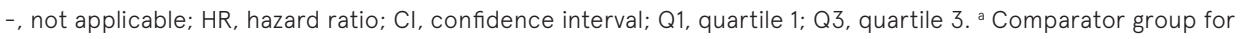
HR. * Significant difference at $P<0.05$.

\section{DISCUSSION}

This study evaluated the compliance and persistence to commonly prescribed ULT drugs for the treatment of gout and hyperuricemia, as well as factors associated with these, on a national scale. 
A

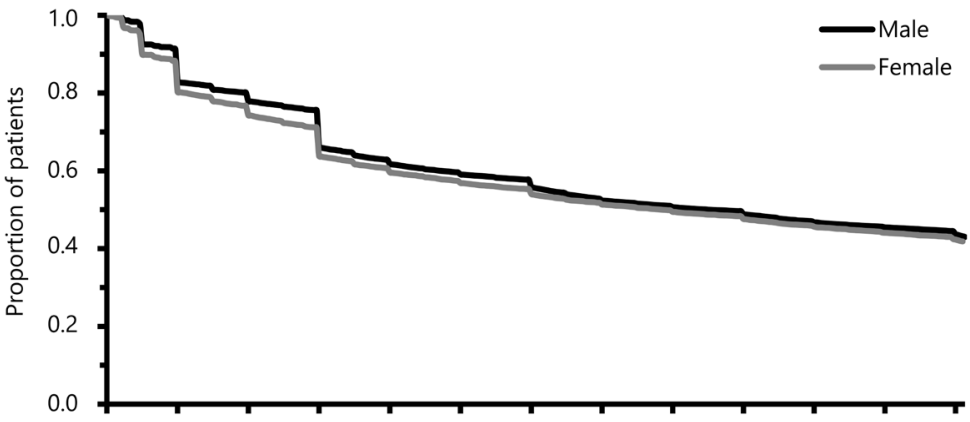

B

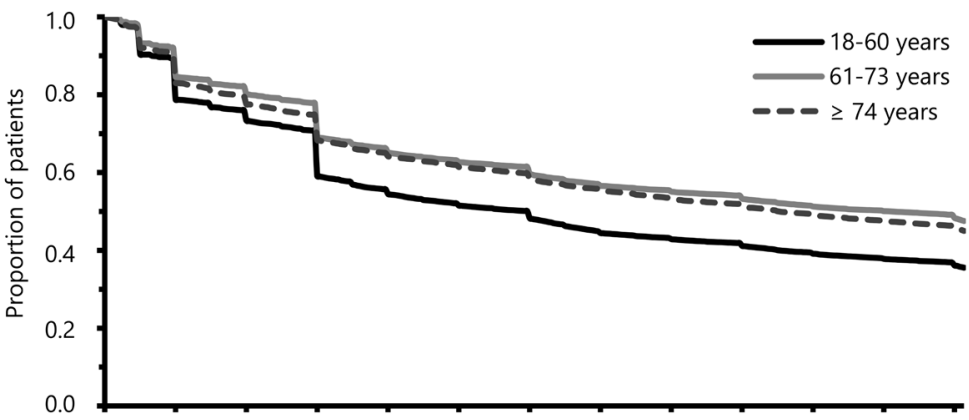

C

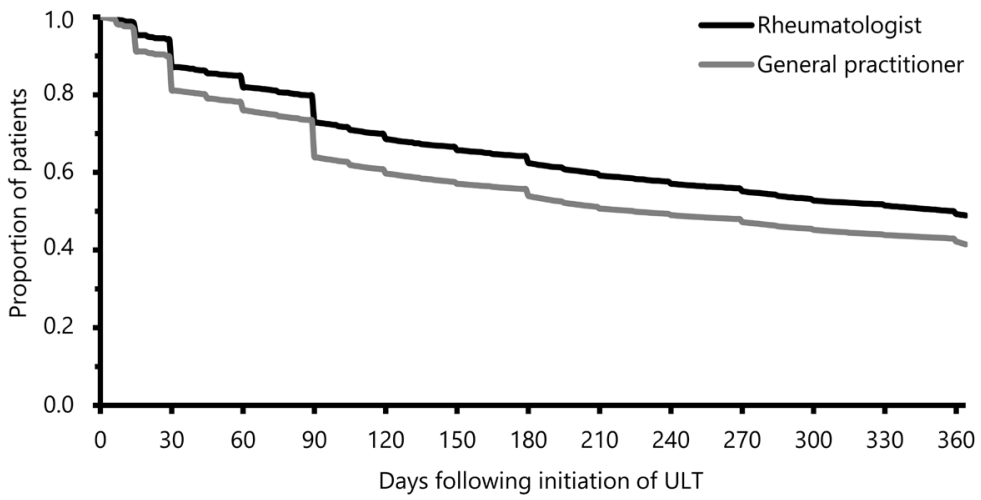

Fig. 1 Graphs of the proportion of patients who continued treatment with urate-lowering therapy (ULT) 1 year after initiation of treatment. A The level of continuance stratified by gender, B stratified by age, and $\mathbf{C}$ stratified by initial prescriber of ULT.

Based on our results, compliance and persistence to ULT for the treatment of gout and hyperuricemia seems suboptimal in the Netherlands, revealing ULT dispensing patterns that are inconsistent with national and international management guidelines 
for gout. After 1 year, only $42.7 \%$ of the patients were still getting their prescriptions refilled, and $51.7 \%$ of the patients had ULT medication coverage of at least $80 \%$ of the study days. These findings further substantiate previous reports where suboptimal medication adherence to ULT has been highlighted as a concern in gout patients, in various healthcare systems ${ }^{[6,13-15]}$. In fact, an earlier report from Germany and the United Kingdom described similar discouraging results ${ }^{[16]}$. Although we can only speculate on the reasons for poor adherence, we found that patients whose initial prescriber of ULT was a rheumatologist had better treatment adherence. This suggests that establishing local networks between rheumatologists and general practitioners, as previously suggested in an editorial in this journal, may be helpful for enhancing gout care ${ }^{[17]}$. Such initiatives, and in particular their influence on medication adherence, should be investigated in The Netherlands.

We found that increasing age, being male, and initiation of ULT by a rheumatologist were factors associated with compliance and continuation of ULT, although it should be noted that these variables explained only a limited amount of the total variance in PDC scores. Nevertheless, the findings that increasing age and male sex were protective factors are consistent with previous research ${ }^{[18]}$. Moreover, our results show a comparatively strong protective effect for the initial prescriber of ULT with respect to compliance, which is a factor that has thus far been studied in a limited number of studies ${ }^{[19]}$.

An important strength of the study is the large, national coverage provided by the database, and the large number of patient records evaluated. It suggests that the presented results may adequately reflect the adherence levels of Dutch patients to ULT. However, our study also had some limitations. First, considering the definitions we maintained for compliance, the PDC was calculated for each patient over the course of the study year. This means the PDC scores after 1 year were to a large extent determined by the level of persistence of that patient in that same year. Furthermore, our database did not contain information regarding the clinical diagnosis of disease for which ULT was indicated. As such, patients without the diagnosis gout and only indicated for hyperuricemia could have been included, potentially introducing bias to the results. Nevertheless, by applying our patient selection criteria, the probability that the database contained gout patients increased. Lastly, we applied a 0.80 cut-off point for good compliance to facilitate comparison with earlier studies. However, it is currently not known if this cut-off value is clinically relevant for gout patients on ULT. As done for other diseases, we suggest empirical evidence supporting the optimal level of adherence should become available for gout and hyperuricemia, to assist in interpreting compliance levels found among patients on ULT [20]. 
In conclusion, our results from real-life prescription data show that medication compliance and persistence to ULT for the treatment of gout and hyperuricemia is suboptimal, and not in line with management guidelines for gout recommending lifelong ULT. Future studies addressing the reasons for treatment cessation and improving treatment adherence are warranted.

\section{Acknowledgements}

We thank C. Widrich BSC and K. He MSc from IQVIA, Real World Evidence Solutions, Amsterdam, The Netherlands, for their assistance in gaining insight in, and understanding the database. 


\section{REFERENCES}

1. Zhu Y, Pandya BJ, Choi HK. Prevalence of gout and hyperuricemia in the US general population: The National Health and Nutrition Examination Survey 2007-2008. Arthritis Rheum. 2011;63(10):3136-41.

2. Khanna D, Fitzgerald JD, Khanna PP, Bae S, Singh MK, Neogi T, et al. 2012 American college of rheumatology guidelines for management of gout. part 1: Systematic nonpharmacologic and pharmacologic therapeutic approaches to hyperuricemia. Arthritis Care Res. 2012;64(10):1431-46.

3. Becker MA, Schumacher HR, Benjamin KL, Gorevic P, Greenwald M, Fessel J, et al. Quality of life and disability in patients with treatment-failure gout. J Rheumatol. 2009;36(5):1041-8.

4. Roddy E. Revisiting the pathogenesis of podagra: why does gout target the foot? J Foot Ankle Res. 2011;4(1):13.

5. Richette P, Doherty M, Pascual E, Barskova V, Becce F, Castañeda-Sanabria J, et al. 2016 updated EULAR evidence-based recommendations for the management of gout. Ann Rheum Dis. 2017;76(1):29-42.

6. Doherty M, Jansen TL, Nuki G, Pascual E, Perez-Ruiz F, Punzi L, et al. Gout: why is this curable disease so seldom cured? Ann Rheum Dis. 2012;71(11):1765-70.

7. Spencer K, Carr A, Doherty M. Patient and provider barriers to effective management of gout in general practice: a qualitative study. Ann Rheum Dis. 2012;71(9):1490-5.

8. Kuo C-F, Grainge MJ, Mallen C, Zhang W, Doherty M. Rising burden of gout in the UK but continuing suboptimal management: a nationwide population study. Ann Rheum Dis. 2015;74(4):661-7.

9. Nasser-Ghodsi N, Harrold LR. Overcoming adherence issues and other barriers to optimal care in gout. Curr Opin Rheumatol. 2015;27(2):134-8.

10. Scheepers LEJM, van Onna M, Stehouwer CDA, Singh JA, Arts ICW, Boonen A. Medication adherence among patients with gout: A systematic review and meta-analysis. Semin Arthritis Rheum. 2018;47(5):689-702.

11. Peterson A, Nau DP, Cramer J, Benner J, Gwadry-Sridhar F, Nichol M. A Checklist for Medication Compliance and Persistence Studies Using Retrospective Databases. Value Heal. 2007;10(1):3-12.

12. Cribari-Neto F, Zeileis A. Beta Regression in R. J Stat Softw. 2010;34(2):1-24.

13. Chia FLA. Poorly controlled gout: Who is doing poorly? Singapore Med J. 2016;57(8):412-4.

14. Becker MA, Chohan S. We can make gout management more successful now. Curr Opin Rheumatol. 2008;20(2):167-72.

15. McGowan B, Bennett K, Silke C, Whelan B. Adherence and persistence to urate-lowering therapies in the Irish setting. Clin Rheumatol. 2016;35(3):715-21.

16. Annemans L, Spaepen E, Gaskin M, Bonnemaire M, Malier V, Gilbert T, et al. Gout in the UK and Germany: prevalence, comorbidities and management in general practice 2000-2005. Ann Rheum Dis. 2008;67(7):960-6.

17. Jansen TL, Janssen M. The American College of Physicians and the 2017 guideline for the management of acute and recurrent gout: treat to avoiding symptoms versus treat to target. Clin Rheumatol. 2017;36(11):2399-402.

18. De Vera MA, Marcotte G, Rai S, Galo JS, Bhole V. Medication adherence in gout: A systematic review. 
Arthritis Care Res. 2014;66(10):1551-9.

19. Solomon DH, Avorn J, Levin R, Brookhart MA. Uric acid lowering therapy: prescribing patterns in a large cohort of older adults. Ann Rheum Dis. 2008;67(5):609-13.

20. Karve S, Cleves MA, Helm M, Hudson TJ, West DS, Martin BC. Good and poor adherence: optimal cut-point for adherence measures using administrative claims data. Curr Med Res Opin. 2009;25(9):2303-10. 



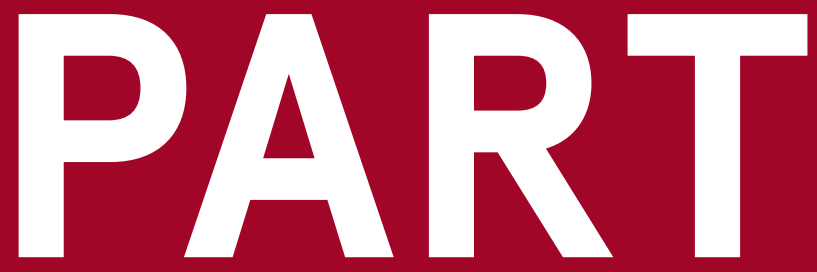

Patient-reported outcome measures in gout 



\section{A systematic literature review of patient-reported outcome measures used in gout: an evaluation of their content and measurement properties}

Carly A. Janssen, Martijn A.H. Oude Voshaar, Peter M. ten Klooster, Tim L.Th.A. Jansen, Harald E. Vonkeman, Mart A.F.J. van de Laar 


\section{ABSTRACT}

Background. Gout is a common, monosodium urate crystal-driven inflammatory arthritis. Besides its clinical manifestations, patients often also suffer from pain, physical impairment, emotional distress and work productivity loss, as a result of the disease. Patient-reported outcome measures (PROMs) are commonly used to assess these consequences of the disease. However, current instrument endorsements for measuring such outcomes in acute and chronic gout clinical settings are based on limited psychometric evidence. The objective of this systematic literature review was to identify currently available PROMs for gout, and to critically evaluate their content and psychometric properties, in order to evaluate the current status regarding PROMs for use in gout patients.

Methods. Systematic literature searches were performed in the PubMed and EMBASE databases. The methodological quality of included papers was appraised using the COnsensus-based Standards for the selection of health Measurement INstruments (COSMIN) checklist, and evaluation of measurement properties (reliability, responsiveness, construct validity, floor and ceiling effects) was done in accordance with published quality criteria. Item content was appraised by linking health concepts to the International Classification of Functioning Disability and Health (ICF) framework.

Results. In total, 13 PROMs were identified, of which three were targeted specifically at gout patients. The majority of the PROMs were rated positively for content validity. For most instruments, limited evidence was available for construct validity and reliability. Instruments to assess pain scored well on responsiveness and floor and ceiling effects, but not much is known about their reliability in gout.

Conclusions. The physical functioning subscale of the SF-36v2 (Short Form-36 item version 2) is the only PROM that had sufficient supporting evidence for all its psychometric properties. Many of the commonly used PROMs in gout are currently not yet well supported and more studies on their measurement properties are needed among both acute and chronic gout populations. 


\section{INTRODUCTION}

Gout is an increasingly prevalent, monosodium urate crystal-driven inflammatory arthritis, commonly presenting as debilitating acute painful flares with associated redness and swelling of the affected joint(s). In some cases a chronic course may develop when increasing crystal deposition is left untreated, leading to visible urate crystal deposits (tophi) and joint damage, as well as extra-articular complications ${ }^{[1]}$. Along with the clinical manifestations, patients suffering from gout are often confronted with pain, physical impairment, work productivity loss, and emotional distress ${ }^{[2,3]}$. Patient-reported outcome measures (PROMs) are commonly used to assess these consequences of gout in a variety of settings ${ }^{[4,5]}$.

When choosing a specific PROM to use from a number of alternatives, one should take into account the research context, feasibility of the instrument, comparability of scores with relevant earlier work, and the measurement properties of the instrument in the population of interest. Measurement properties are arguably a particularly important factor to consider, since they have a direct bearing on, for example, the ability of a study to demonstrate the desired effects, as well as the required sample size. Therefore, choosing the best instrument from a number of alternatives importantly contributes to the potential for the success of a study. Consequently, endorsements of specific instruments should be based on a comprehensive, critical evaluation of their content and the documented evidence supporting their measurement properties ${ }^{[6]}$.

The OMERACT Gout Special Interest Group has endorsed various patient-reported outcome (PRO) instruments for use in acute and chronic gout clinical research ${ }^{[7-13]}$. However, these endorsements are based only on the opinions of experts, guided by analyses performed on data from a few selected clinical trials $(n=4)$ and one observational study, as well as a systematic review on the performance of specific measures in previous clinical trials of acute gout ${ }^{[14,15]}$. Important measurement properties, such as reliability and validity, are not typically reported on in trial reports, nor can information about these properties necessarily be inferred from the reported results. Also, as information about measurement properties was derived from a small, selected number of studies, new or less popular instruments may have been underappreciated.

To date, no systematic evaluation has been performed of the available evidence supporting the measurement properties of the various PROMs available for use in gout ${ }^{[16,17]}$. The objective of this systematic review was to identify all PROMs currently available for gout, and to critically appraise their content and measurement properties, in order 
to evaluate the current status regarding PROMs validated for use in gout patients, and to identify areas for future research.

\section{METHODS}

\section{Search strategy}

To identify all available literature, a systematic literature search was performed in PubMed and EMBASE (database start date, up to August 15, 2017), using a modified, but validated search strategy for papers on measurement properties of PROMs used in gout ${ }^{[18]}$. The exact search terms are included in the supplemental material (Supplemental material 1). References of included studies and systematic reviews of PROMs found in the search were screened initially by title, and if relevant, abstracts were assessed for potentially relevant papers. Finally, for each included PROM a PubMed search was performed to make sure all papers were included.

\section{Selection of literature}

Inclusion criteria were published articles in which (1) the study population consisted of gout patients and (2) the article reported on the development of a PROM, or the evaluation of one or more of its measurement properties. We excluded (1) conference abstracts and poster presentations, (2) systematic review articles, and (3) articles published in any other language than English.

The titles and abstracts of the retrieved articles were screened independently by two reviewers (MOV and CJ) on relatedness to gout and development or evaluation of a PROM. Any duplicates of articles generated by the search strategy were removed using Microsoft Excel prior to screening. When the title or abstract caused uncertainty pertaining the eligibility criteria, the full-text articles were retrieved and assessed. Disagreements on the eligibility of the article for inclusion were discussed and resolved through consensus. A third reviewer (PtK) was consulted if disagreements remained unresolved. Full-text articles were retained and the final decision on which studies to include were made through consensus after having read the articles (MOV and PtK). Reasons for exclusion were noted and a flow chart of study article selection was prepared according to the Preferred Reporting Items for Systematic Reviews and Meta-Analyses statement ${ }^{[19]}$.

\section{PROM characteristics}

Descriptive characteristics of each instrument were extracted from the included studies or the initial publication of the instrument. The readability of each questionnaire was 
assessed using the Flesh-Kincaid Grade level-test. A grade level of 6 is recommended by the International Society for Quality of Life Research (ISOQOL) minimum standards for PROMs ${ }^{[20]}$. Availability of each instrument was determined.

\section{Assessment of methodological quality}

The methodological quality of each included study was assessed using the COnsensusbased Standards for the selection of health Measurement INstruments (COSMIN) checklist [21]. Several deviations from COSMIN checklist were deemed necessary in order to correspond better with advances in psychometric theory or standard practices in gout, and quality of life research ${ }^{[20,22-26]}$. An overview of the criteria and our deviations from these are presented in Table 1. Two reviewers (MOV and PtK) independently completed the checklist and final decisions about ratings were arrived at through consensus.

Table 1: Quality criteria for rating the measurement properties in accordance with Terwee et al. 2007, and deviations from COSMIN criteria for methodological quality.

\begin{tabular}{|c|c|c|c|}
\hline $\begin{array}{l}\text { Measurement } \\
\text { property }^{a}\end{array}$ & Definition & $\begin{array}{l}\text { Deviations from COSMIN } \\
\text { checklist }\end{array}$ & $\begin{array}{l}\text { Threshold for } \\
\text { positive rating }\end{array}$ \\
\hline $\begin{array}{l}\text { Score reliability } \\
\text { (single } \\
\text { administration) }\end{array}$ & $\begin{array}{l}\text { Classical test theory } \\
\text { based estimate of overall } \\
\text { proportion of true score } \\
\text { variance, calculated from } \\
\text { the interitem covariance } \\
\text { matrix. }\end{array}$ & $\begin{array}{l}\text { Following Sijtsma et al. } \\
2009, \text { the term "internal } \\
\text { consistency" was replaced } \\
\text { by single administration } \\
\text { reliability. Single } \\
\text { administration reliability } \\
\text { coefficients were considered } \\
\text { to provide information about } \\
\text { score reliability. }\end{array}$ & $\begin{array}{l}\text { Reliability } \\
\text { coefficient } \geq 0.70 \text {. }\end{array}$ \\
\hline $\begin{array}{l}\text { Score reliability } \\
\text { (test-retest) }\end{array}$ & $\begin{array}{l}\text { Classical test theory } \\
\text { based estimate of overall } \\
\text { proportion of true score } \\
\text { variance, obtained from } \\
\text { the correlation between } \\
\text { repeated measures with } \\
\text { same instrument in stable } \\
\text { patients. }\end{array}$ & $\begin{array}{l}\text { None, but single } \\
\text { administration and test- } \\
\text { retest reliability categories } \\
\text { were merged in the } \\
\text { measurement properties } \\
\text { appraisal. }\end{array}$ & $I C C \geq 0.70$ \\
\hline $\begin{array}{l}\text { Construct } \\
\text { Validity }\end{array}$ & $\begin{array}{l}\text { The degree to which PRO } \\
\text { scores are related to } \\
\text { scores of other validated } \\
\text { measures in a way that is } \\
\text { consistent with theories } \\
\text { about how the constructs } \\
\text { the measures presume to } \\
\text { assess, are related. }\end{array}$ & None. & $\begin{array}{l}\text { At least } 75 \% \text { of } \\
\text { the results are in } \\
\text { accordance with } \\
\text { the hypotheses. }\end{array}$ \\
\hline
\end{tabular}


Table 1 continued.

\begin{tabular}{|c|c|c|c|}
\hline $\begin{array}{l}\text { Measurement } \\
\text { property }^{\mathrm{a}}\end{array}$ & Definition & $\begin{array}{l}\text { Deviations from COSMIN } \\
\text { checklist }\end{array}$ & $\begin{array}{l}\text { Threshold for } \\
\text { positive rating }\end{array}$ \\
\hline $\begin{array}{l}\text { Floor and } \\
\text { Ceiling effects }\end{array}$ & $\begin{array}{l}\text { The number of } \\
\text { respondents who } \\
\text { achieved the lowest or } \\
\text { highest possible score. }\end{array}$ & None. & $\begin{array}{l}\leq 15 \% \text { of the } \\
\text { respondents } \\
\text { achieved the } \\
\text { highest or lowest } \\
\text { possible score. }\end{array}$ \\
\hline Responsiveness & $\begin{array}{l}\text { The extent to which a } \\
\text { PROM can detect changes } \\
\text { in the construct being } \\
\text { measured over time. }\end{array}$ & $\begin{array}{l}\text { Following the ISOQOL } \\
\text { recommendations and } \\
\text { Revicky et al. 2008, favorable } \\
\text { rating for responsiveness } \\
\text { required empirical evidence } \\
\text { of changes in scores } \\
\text { consistent with a priori } \\
\text { expectations of researchers; } \\
\text { Either evidenced by score } \\
\text { improvement following } \\
\text { intervention with known } \\
\text { efficacy, or score changes } \\
\text { in accordance with } \\
\text { expectations derived from } \\
\text { external anchors of change } \\
\text { (e.g. patient-reported } \\
\text { changed overall health } \\
\text { status). }\end{array}$ & $\begin{array}{l}\text { Standardized } \\
\text { change scores of } \\
\text { at least moderate } \\
\text { magnitude (e.g., } \\
\text { ES / SRM } \geq 0.30 \\
\text { in the expected } \\
\text { direction if } \\
\text { changes were } \\
\text { expected). }\end{array}$ \\
\hline
\end{tabular}

a For all boxes the reporting standards on missing data were ignored when appraising methodological quality, because such information was rarely reported.

PROMs, patient-reported outcome measures; COSMIN, consensus-based standards for the selection of health measurement instruments; ISOQOL, international society for quality of life research; ICC, intraclass correlation; PRO, patient-reported outcome; ES, effect size; SRM, standardized response mean.

\section{Assessment of measurement properties}

The studies that were judged to be of high methodological quality using the COSMIN checklist were used to rate the measurement properties of the included PROM as either good (+) or poor (-), in accordance with quality criteria proposed by Terwee et al. $2007^{[26]}$. Measurement properties of instruments for which only studies of insufficient methodological quality were available were rated as indeterminate (?), or zero (0) when no information was found for that measurement property. In cases where the same PROM was described in various studies of sufficient methodological quality, which resulted in different quality ratings for the same measurement property, the rating was designated as indecisive (+/-). Table 1 gives a description of each rated measurement property, along with the quality criteria applied. 


\section{Content evaluation and assessment of content validity}

Health concepts assessed by each PROM were characterized by linking the items to the International Classification of Functioning, disability and Health (ICF) using the 2016 ICF linking rules ${ }^{[27]}$. As our intention was to compare content between PROMs, we did not link health concepts to the 'other specified' or 'unspecified' ICF categories. To be rated as having a high content validity $(+), \geq 75 \%$ of the health concepts of the PROM had to have been included in either ICF core set ${ }^{[28,29]}$. All health items related to emotions were linked to the ICF category 'b152 emotional functions'.

\section{Item response theory (IRT)}

Although no quality criteria are currently available to judge the quality of studies that used IRT-based analysis, we provided a descriptive review of the results of the included studies that used these methods. As a minimum requirement for methodological quality of the studies, we required that at least 50 patients were included in the study for each item in case of PROMs with dichotomous response categories, or 50 patients for each item step parameter for polytomous data ${ }^{[30]}$. For articles that used 2-parameter IRT modeling we required a minimum of 250 patients to be included ${ }^{[31]}$. Furthermore, for a positive rating for methodological quality, the IRT model that was used should be described in sufficiently detail for the reader to understand its parametric structure, or references needed to be included to sources that provide such descriptions. Finally, at least some evidence needed to be presented to support model-data fit.

\section{RESULTS}

The search resulted in 826 hits, of which 556 were screened after removal of the duplicates. After screening of the titles and abstracts, 33 were found to meet the inclusion criteria. Of these, another 19 were excluded, leaving a total of 14 studies for review (Fig. 1) ${ }^{[15,32-44]}$. Reference checking of systematic reviews and of these included papers, or the additional PubMed search of each included instrument, did not result in any additional studies eligible for review.

\section{Instrument characteristics}

The characteristics of the 13 included PROMs are summarized in Table 2 (see Supplemental material 2 for ICF linking perspectives and categorization of response options). Three of these specifically target gout patients, whereas the remaining instruments target patients with rheumatic diseases or generic populations. Pain and physical function were the most frequently assessed outcome domains. Seven out of eleven (64\%) PROMs 
had Flesch-Kincaid grade level estimates lower than 6, suggesting that their items are easily understood by patients with varying reading proficiency levels (Table 2).

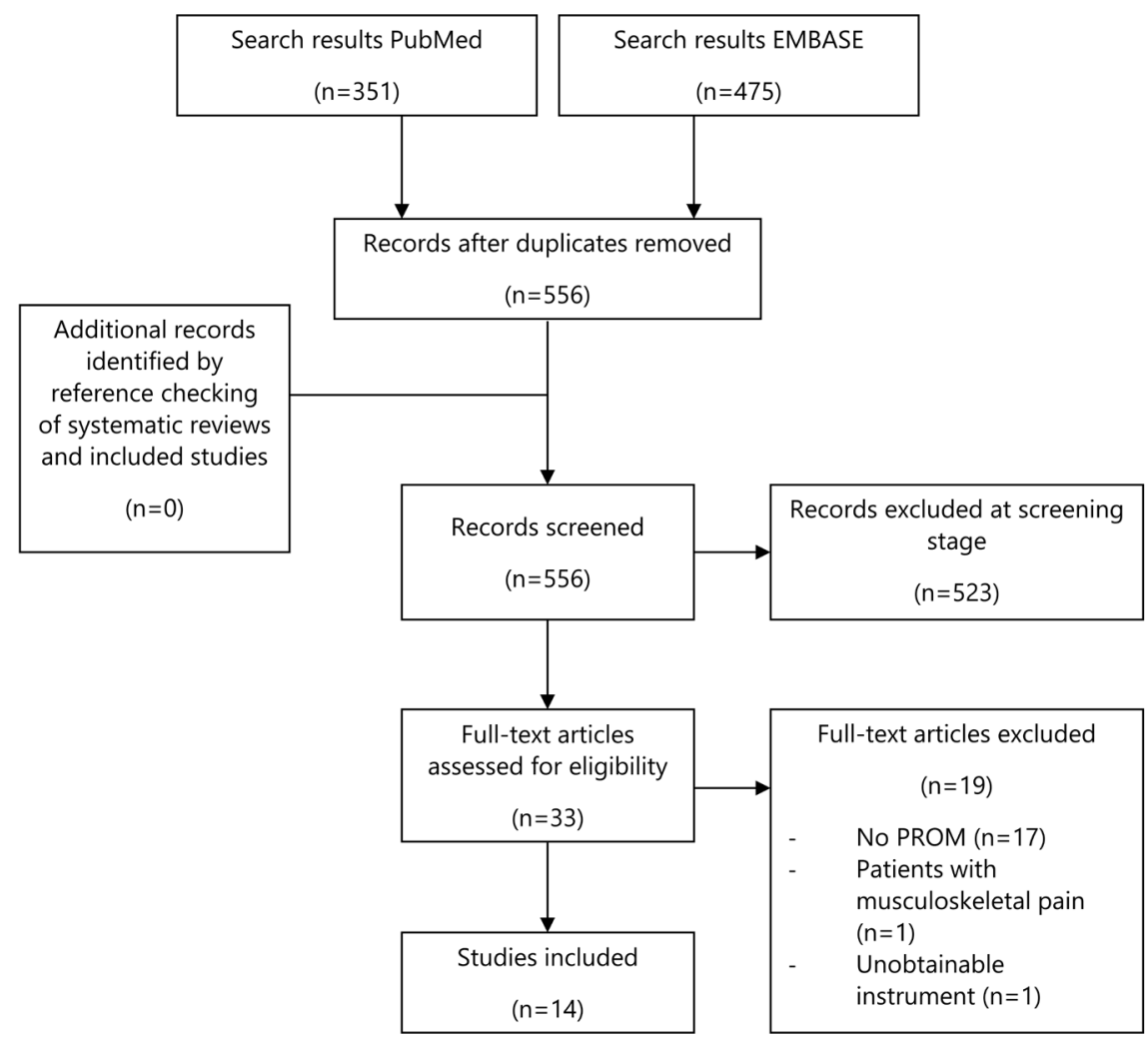

Fig. 1 Flow diagram of study selection

\section{Content description \& validity}

The results of the ICF-linking exercise revealed that health concepts subsumed under the ICF chapter 'd4 mobility' were most frequently addressed in the items of the included PROMs (see Supplemental material 3). Each included PROM had at least one item related to ' $\mathrm{d} 4$ mobility'. The 'b1 mental functions' was the second most popular ICF chapter. This is because all health concepts related to emotional functioning were linked to this chapter. Health concepts related to 'd8 major life areas' were also frequently assessed, mainly due to the inclusion of the Rheumatoid Arthritis-Work Instability Scale (RA-WIS). Only three PROMs included content related to environmental factors, 
particularly the Health Assessment Questionnaire-Disability Index (HAQ-DI), for which scores can be adjusted in case patients need help from others or assistive devices to perform the activities.

Table 2: General characteristics of the included patient-reported outcome measures (PROMs)

\begin{tabular}{|c|c|c|c|c|c|c|}
\hline \multirow[b]{2}{*}{ Instrument } & \multirow[b]{2}{*}{$\begin{array}{l}\text { Target } \\
\text { population }\end{array}$} & \multirow[b]{2}{*}{ Subscale (number of items) } & \multicolumn{2}{|c|}{$\begin{array}{l}\text { OMERACT } \\
\text { core outcome } \\
\text { domains }\end{array}$} & \multicolumn{2}{|c|}{ Feasibility } \\
\hline & & & $\begin{array}{l}\text { Acute } \\
\text { gout }^{\text {a }}\end{array}$ & $\begin{array}{l}\text { Chronic } \\
\text { goutb }^{\text {b }}\end{array}$ & $\begin{array}{l}\text { Read- } \\
\text { abilityc }^{c}\end{array}$ & Availability \\
\hline \multicolumn{7}{|c|}{ Multidimensional scales } \\
\hline $\begin{array}{l}\text { SF-36v2 } \\
{[34-37,41,43,44]}\end{array}$ & Generic & $\begin{array}{l}\text { Physical functioning (10), role- } \\
\text { physical (4), bodily pain (2), } \\
\text { general health (5), vitality (4), } \\
\text { social functioning (2), role- } \\
\text { emotional (3), mental health (5) }\end{array}$ & $\begin{array}{l}P \\
P G A \\
A L\end{array}$ & $\begin{array}{l}\text { HRQOL, } \\
\text { P, PGA, } \\
\text { AL }\end{array}$ & 5.6 & $\begin{array}{l}\text { License fee } \\
\text { may apply }\end{array}$ \\
\hline MOS-20 $0^{[33]}$ & Generic & $\begin{array}{l}\text { Physical function (6), } \\
\text { role functional (2), social } \\
\text { functioning (1), mental health } \\
\text { (5), current perception of } \\
\text { health (5), pain (1) }\end{array}$ & $\begin{array}{l}P \\
P G A \\
A L\end{array}$ & $\begin{array}{l}\text { HRQOL, } \\
\text { P, PGA, } \\
\text { AL }\end{array}$ & 6.5 & $\begin{array}{l}\text { Freely } \\
\text { available }\end{array}$ \\
\hline $\mathrm{AIMS}^{[33]}$ & Arthritis & $\begin{array}{l}\text { Mobility (4), physical activity } \\
\text { (5), dexterity (5), household } \\
\text { activity (7), social activities (4), } \\
\text { activities of daily living (4), pain } \\
\text { (4), depression (6), anxiety (6) }\end{array}$ & $P, A L$ & $\begin{array}{l}\text { HRQOL, } \\
P, A L\end{array}$ & 5.6 & $\begin{array}{l}\text { Freely } \\
\text { available }\end{array}$ \\
\hline $\begin{array}{l}\text { GAQ } \\
2.0^{[34,41,43]}\end{array}$ & Gout & $\begin{array}{l}\text { GIS (24), consists of } 5 \\
\text { subscales: gout concern } \\
\text { overall (4), gout medication } \\
\text { side effects ( } 2 \text { ), unmet gout } \\
\text { treatment need (3), well-being } \\
\text { during attack (11), gout concern } \\
\text { during attack (4) }\end{array}$ & PGA & $\begin{array}{l}\text { AGA, } \\
\text { HRQOL, } \\
\text { P.PGA }\end{array}$ & $7.2^{\mathrm{d}}$ & $\begin{array}{l}\text { Freely } \\
\text { available }\end{array}$ \\
\hline \multicolumn{7}{|c|}{ Unidimensional scales } \\
\hline $\begin{array}{l}\mathrm{HAQ-} \\
\mathrm{DI}^{[15,32,33,37-41,43]}\end{array}$ & Generic & $(43)^{e}$ & $A L$ & $\mathrm{AL}$ & 4.6 & $\begin{array}{l}\text { Freely } \\
\text { available }\end{array}$ \\
\hline $\mathrm{HAQ}-\|^{[15,36,37]}$ & $\begin{array}{l}\text { Rheumatic } \\
\text { conditions }\end{array}$ & $(10)$ & $\mathrm{AL}$ & $A L$ & 4.0 & $\begin{array}{l}\text { Freely } \\
\text { available }\end{array}$ \\
\hline $\mathrm{TIQ}-2 \mathrm{O}^{[42]}$ & $\begin{array}{l}\text { Tophaceous } \\
\text { gout }\end{array}$ & $(20)$ & - & $\mathrm{TB}$ & 5.6 & $\begin{array}{l}\text { Freely } \\
\text { available }\end{array}$ \\
\hline RA-WIS ${ }^{[36]}$ & RA & (23) & - & - & 3.7 & $\begin{array}{l}\text { License fee } \\
\text { may apply }\end{array}$ \\
\hline \multicolumn{7}{|c|}{ Single-item PROMs } \\
\hline VAS pain ${ }^{\mathrm{f}[15,35]}$ & Multiple & (1) & $\mathrm{P}$ & $P$ & $3.3^{\mathrm{g}}$ & $\begin{array}{l}\text { Freely } \\
\text { available }\end{array}$ \\
\hline $\begin{array}{l}\text { Likert pain } \\
\text { [15] }\end{array}$ & Multiple & (1) & $P$ & $P$ & $\mathrm{n} / \mathrm{a}$ & $\begin{array}{l}\text { Freely } \\
\text { available }\end{array}$ \\
\hline
\end{tabular}


Table 2 continued.

\begin{tabular}{|c|c|c|c|c|c|c|}
\hline \multirow[b]{2}{*}{ Instrument } & \multirow[b]{2}{*}{$\begin{array}{l}\text { Target } \\
\text { population }\end{array}$} & \multirow[b]{2}{*}{ Subscale (number of items) } & \multicolumn{2}{|c|}{$\begin{array}{l}\text { OMERACT } \\
\text { core outcome } \\
\text { domains }\end{array}$} & \multicolumn{2}{|c|}{ Feasibility } \\
\hline & & & $\begin{array}{l}\text { Acute } \\
\text { gout }^{\text {a }}\end{array}$ & $\begin{array}{l}\text { Chronic } \\
\text { goutb }^{\text {got }}\end{array}$ & $\begin{array}{l}\text { Read- } \\
\text { abilityc }^{c}\end{array}$ & Availability \\
\hline \multicolumn{7}{|c|}{ Single-item PROMs } \\
\hline NRS pain ${ }^{i[15]}$ & Multiple & (1) & $\mathrm{P}$ & $P$ & $\mathrm{n} / \mathrm{a}$ & $\begin{array}{l}\text { Freely } \\
\text { available }\end{array}$ \\
\hline VAS PGAj ${ }^{j}[15,35]$ & Multiple & (1) & PGA & PGA & $11.9^{k}$ & $\begin{array}{l}\text { Freely } \\
\text { available }\end{array}$ \\
\hline $\begin{array}{l}\text { Physical } \\
\text { function NRS' } \\
\text { [15] }\end{array}$ & Gout & (1) & $A L$ & $A L$ & $12.7^{\mathrm{m}}$ & $\begin{array}{l}\text { Freely } \\
\text { available }\end{array}$ \\
\hline
\end{tabular}

aOMERACT mandatory core outcome domains for acute gout are pain (P), joint swelling (JS), joint tenderness (JT), patient global assessment (PGA), activity limitation (AL) [10]. 'OMERACT mandatory core outcome domains for chronic gout: serum uric acid (sUA), acute gout attack (AGA), tophus burden (TB), Health-related quality of life (HRQOL), activity limitations (AL), pain (P), patient global assessment (PGA) ${ }^{[10]}$. 'A Flesch-Kincaid Grade Level score of $\leq 6$ was desired, equivalent to 6th-grade education level or lower in the United States (12 years or lower). dRated for the GIS section of the GAQ2.0 only. eBased upon the HAQ-DI Dutch consensus ${ }^{[45] .}{ }^{\text {f } 100 m m ~ V A S ~}(0=$ no

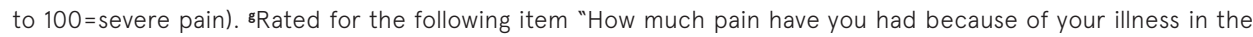
past week?" [35]. "h5-point Likert scale ( $0=$ no pain, $1=$ mild pain, $2=$ moderate pain, $3=$ severe pain, 4 =extreme pain). ${ }^{i} 11$-point NRS ( $0=$ no pain to $10=$ extreme pain). ${ }^{j} 100 \mathrm{~mm}$ VAS $\left(0=\right.$ very well to $100=$ very poor). ${ }^{k}$ Rated for the following item "Considering all the ways that your arthritis affects you, rate how you are doing today on the following scale by placing a vertical mark on the line" [35]. 111-point NRS from WPAI:SHP v2.0 ( 0 =had no effect on my daily activities to $100=$ completely prevented me from doing my daily activities) ${ }^{[46]}$. ${ }^{m}$ Rated for the following questionnaire item "During the past seven days, how much did your gout attack affect your ability to do your regular daily activities, other than work at a job?".

PROMs, patient-reported outcome measures; OMERACT, outcome measures in rheumatology; RA, rheumatoid arthritis; SF-36v2, Short Form-36 item version 2; MOS-20, Medical Outcomes Study 20-item Short Form Health Survey; AIMS, Arthritis Impact Measurement Scales; GAQ 2.0, Gout Assessment Questionnaire 2.0; GIS, Gout Impact Scale; HAQ-DI, Health Assessment Questionnaire-Disability Index; HAQ-II, Health Assessment Questionnaire-II; TIQ-20, 20-item Tophus Impact Questionnaire; RA-WIS, Rheumatoid Arthritis-Work Instability Scale; VAS, Visual Analogue Scale; NRS, Numeric Rating Scale; PGA, patient global assessment; - , not applicable; $\mathrm{n} / \mathrm{a}$, not available

Of the in total 32 PROMs, subscales and total scales that were rated, $81 \%(n=26)$ met the criteria for a positive rating for content validity (Table 3). However, the role functioning subscales of Medical Outcomes Study 20-item Short Form Health Survey (MOS20) and Short Form-36 item version 2 (SF-36v2), the Work Productivity and Activity Impairment (WPAI) physical function Numeric Rating Scale (NRS) and several Arthritis Impact Measurement Scales (AIMS) subscales received negative ratings, mainly due to the fact that a large number of their health concepts were too general to be linked to ICF second level categories. 


\section{Quality rating of measurement properties}

Table 3 lists the quality ratings of the psychometric properties of the included PROMs.

\section{Construct validity}

The methodological quality for construct validity was frequently rated as poor, in the majority of cases because no hypotheses were specified by the authors with respect to expected correlations or mean differences. In studies with explicitly stated hypotheses, positive ratings were generally given, leading to mostly positive ratings for PROMs for which high quality studies of construct validity were available. However, the HAQ-DI was rated as inconclusive as in one study, $78 \%$ of hypotheses were confirmed, whereas in another only $61 \%$ of hypotheses could be confirmed. For the latter study, hypotheses were not confirmed for some correlations with the subscales of the SF-36v2 (including emotional health, emotional role limitation, social), but also for correlations with outcomes such as the number of gout flares in the past month, Visual Analogue Scale (VAS) for pain, swollen joint count and physician global assessment.

\section{Score reliability}

The reliability of several multi-item PROMs was supported by high quality studies of single administration reliability. All instruments measuring physical function (HAQ-DI, Health Assessment Questionnaire-II (HAQ-II), SF-36v2 physical functioning subscale) received favorable ratings for reliability, as did the RA-WIS and a couple of the Gout Assessment Questionnaire 2.0 (GAQ2.0) subscales and total scale. The other subscales of the GAQ2.0 were either rated negatively because the reliability coefficient was < 0.70 , or as indefinite when studies showed mixed results. The AIMS and MOS-20 were rated as indeterminate because the sample size used for the analysis was inadequate $(<50)$. None of the studies in which an analysis of test-retest reliability was performed were rated to be of high quality. The 20-item Tophus Impact Questionnaire (TIQ-20) was rated as indeterminate for test-retest despite an intraclass correlation coefficient $($ ICC) > 0.70 using an otherwise appropriate design, because patients did not appear stable during the two measurement periods. The AIMS and the MOS-20 received an indeterminate rating because an inadequate sample size was used, and the follow-up period of eight weeks between measurements was deemed too long. For the other six questionnaires, no studies on test-retest reliability were found. 
Table 3: Quality ratings of the measurement properties of the included instruments.

\begin{tabular}{|c|c|c|c|c|c|}
\hline \multirow[b]{2}{*}{ Instrument } & \multicolumn{2}{|l|}{ Truth } & \multicolumn{3}{|c|}{ Discrimination } \\
\hline & $\begin{array}{l}\text { Content } \\
\text { Validity }\end{array}$ & $\begin{array}{c}\text { Construct } \\
\text { validity }\end{array}$ & Reliability & Responsiveness & $\begin{array}{c}\text { Floor and } \\
\text { ceiling effects }\end{array}$ \\
\hline \multicolumn{6}{|l|}{ SF-36v2 ${ }^{[34-37,41,43,44]}$} \\
\hline Physical function & + & + & + & + & + \\
\hline Role physical & - & ? & 0 & + & - \\
\hline Bodily pain & + & + & 0 & + & + \\
\hline General health & $\mathrm{n} / \mathrm{a}$ & $?$ & 0 & + & + \\
\hline Vitality & + & $?$ & 0 & + & + \\
\hline Social functioning & + & $?$ & 0 & + & - \\
\hline Role emotional & + & $?$ & 0 & - & - \\
\hline Mental health & + & $?$ & 0 & - & + \\
\hline SF-36 PCS & $\mathrm{n} / \mathrm{a}$ & $?$ & 0 & + & + \\
\hline SF-36 MCS & $\mathrm{n} / \mathrm{a}$ & $?$ & 0 & - & + \\
\hline \multicolumn{6}{|l|}{ MOS-20 [33] } \\
\hline Physical function & + & $?$ & $?$ & $?$ & 0 \\
\hline Role functioning & - & $?$ & $?$ & $?$ & 0 \\
\hline Social functioning & + & $?$ & $?$ & $?$ & 0 \\
\hline Mental health & + & $?$ & $?$ & $?$ & 0 \\
\hline Health perception & $\mathrm{n} / \mathrm{a}$ & $?$ & $?$ & $?$ & 0 \\
\hline Pain & + & ? & ? & ? & 0 \\
\hline \multicolumn{6}{|l|}{ AIMS [33] } \\
\hline Mobility & + & $?$ & $?$ & $?$ & 0 \\
\hline Physical activity & + & $?$ & $?$ & $?$ & 0 \\
\hline Dexterity & + & $?$ & $?$ & $?$ & 0 \\
\hline Household activities & - & $?$ & $?$ & $?$ & 0 \\
\hline Social activities & - & $?$ & $?$ & $?$ & 0 \\
\hline Activities of daily living & - & $?$ & $?$ & $?$ & 0 \\
\hline Pain & + & $?$ & $?$ & $?$ & 0 \\
\hline Depression & + & $?$ & ? & $?$ & 0 \\
\hline Anxiety & + & $?$ & $?$ & $?$ & 0 \\
\hline \multicolumn{6}{|l|}{ GAQ2.0 $0^{[34,41,43]}$} \\
\hline Concern overall & + & $?$ & $+/-$ & + & + \\
\hline Medication side effects & + & ? & - & - & + \\
\hline Unmet treatment need & + & $?$ & - & - & + \\
\hline Wellbeing during attack & + & $?$ & + & + & + \\
\hline Concern during attack & + & $?$ & + & - & + \\
\hline Total GIS & $\mathrm{n} / \mathrm{a}$ & $?$ & + & + & 0 \\
\hline HAQ-DI $[15,32,33,37-41,43]$ & + & $+/-$ & + & + & $+/-$ \\
\hline HAQ-II ${ }^{[15,36,37]}$ & + & + & + & $?$ & - \\
\hline TIQ-20 & + & + & $?$ & 0 & + \\
\hline RA-WIS ${ }^{[36]}$ & + & + & + & 0 & - \\
\hline VAS pain ${ }^{[15,35]}$ & + & + & $?$ & + & + \\
\hline
\end{tabular}


Table 3 continued.

\begin{tabular}{|c|c|c|c|c|c|}
\hline \multirow[b]{2}{*}{ Instrument } & \multicolumn{2}{|l|}{ Truth } & \multicolumn{3}{|c|}{ Discrimination } \\
\hline & $\begin{array}{l}\text { Content } \\
\text { Validity }\end{array}$ & $\begin{array}{c}\text { Construct } \\
\text { validity }\end{array}$ & Reliability & Responsiveness & $\begin{array}{c}\text { Floor and } \\
\text { ceiling effects }\end{array}$ \\
\hline Likert pain $^{[15]}$ & $\mathrm{n} / \mathrm{d}$ & $?$ & $?$ & + & + \\
\hline NRS pain ${ }^{[15]}$ & $n / d$ & $?$ & 0 & + & + \\
\hline VAS PGA ${ }^{[15,35]}$ & $\mathrm{n} / \mathrm{a}$ & + & 0 & + & + \\
\hline $\begin{array}{l}\text { WPAI Physical function NRS } \\
{[15]}\end{array}$ & - & $?$ & 0 & + & + \\
\hline
\end{tabular}

+ , good measurement property with sufficient methodological quality; +/-, Indefinite measurement property with sufficient methodological quality; - , poor measurement property with sufficient methodological quality; ?, indeterminate quality of measurement properties because of inadequate methodological quality; 0 , no information found in the literature; n/a, not applicable; n/d, not definable due to unavailability of questionnaire item; AIMS, Arthritis Impact Measurement Scales; GAQ 2.0, Gout Assessment Questionnaire 2.0; GIS, Gout Impact Scale; HAQDI, Health Assessment Questionnaire-Disability Index; HAQ-II, Health Assessment Questionnaire-II; MOS-20, Medical Outcomes Study 20-item Short Form Health Survey; SF-36v2, Short Form-36 item version 2; TIQ-20, 20-item Tophus Impact Questionnaire; RA-WIS, Rheumatoid Arthritis-Work Instability Scale; VAS, Visual Analogue Scale; NRS, Numeric Rating Scale; PGA, patient global assessment; WPAI, Work Productivity and Activity Impairment.

\section{Responsiveness}

The single-item pain measures (VAS, Likert and NRS) and the bodily pain subscale of the SF-36v2 were demonstrated to be able to detect clinically relevant changes over time. Of the PROMs measuring physical functioning, the HAQ-DI, SF-36v2 (physical functioning subscale, role physical subscale and the physical component summary score) and the single-item WPAI physical function NRS were rated positively, whereas the HAQ-II was rated as indeterminate because it was not clear how patients changed over time. For the same reason, the MOS-20 and AIMS also received an indeterminate rating. The subscales of the SF-36v2 and GAQ2.0 that were rated negatively did so because the demonstrated effect size was considered too small $(<0.30)$.

\section{Floor and ceiling effects}

The (sub)scale(s) of the gout-specific GAQ2.0 and the TIQ-20 both showed no floor or ceiling effects. Similarly, the pain instruments were also rated positively, as were the patient global assessment VAS and the general health subscale of the SF-36v2. The instruments for physical functioning showed contradictory results: the HAQ-II had floor or ceiling effects $>15 \%$, the HAQ-DI had indecisive results, and the physical functioning subscale of the SF$36 \mathrm{v} 2$ and physical function NRS scale showed no floor or ceiling effects.

\section{Item response theory (IRT)}

There were four articles in which IRT was used. The methodological quality of the first study was rated negatively, because only $\sim 24$ patients per threshold parameter were 
included, which makes it unlikely that the estimates of these parameters, which was the subject of their analysis, were stably estimated ${ }^{[39]}$. In another study, the measurement invariance of the HAQ-DI with respect to diagnosis, was examined ${ }^{[38]}$. Their results suggest that patients with gout, osteoarthritis and rheumatoid arthritis respond differently to the HAQ-DI categories of walking, dressing, and activities. When these differences in response behavior were controlled for in the model, the authors found that the mean disability scores for the different disease groups were changed slightly. This might impact the validity of cross-diagnostic comparisons using the HAQ-DI. Rasch analysis of the RAWIS scale provided support for its unidimensionality ${ }^{[36]}$. Analysis of the locations of the items and persons on the latent measurement continuum revealed that targeting of the scale was supposedly poor, with most of the items clustering together at the middle of the continuum, whereas the distribution of patients was skewed to the right, with a pronounced ceiling effect. Despite this, global reliability was found to be high according to the patient separation index. At last, Rasch analysis was also used in the development of the TIQ-20 ${ }^{[42]}$. That paper was rated negatively for methodological quality because a longitudinal IRT model was apparently used; however it was not described how the dependencies between the repeated measures were taken into account in the analysis.

\section{DISCUSSION}

\section{Brief summary}

In the current study, we identified and critically reviewed the content and psychometric properties of PROMs currently available for gout, using a systematic approach. This paper can be used for determining areas where further research is required for specific PRO domains and measures in gout, especially regarding their measurement properties.

\section{Strengths}

The comprehensive literature search in various databases, as well as the systematic approach applied during this entire review process, are strengths of this study. In addition, this review is the first to critically review various measurement properties of commonly used PROMs in gout, including the assessment of the methodological quality of studies reporting on these measurement properties. For this purpose, standardized criteria were used to assess both the methodological quality of the included studies using the COSMIN checklist, as well as the quality of the measurement properties using quality criteria that were proposed by ISOQOL and Terwee et al. ${ }^{[20,21,26]}$. Furthermore, the content validity of the included PROMs were comprehensively assessed by linking their items to the ICF using standardized ICF linking procedures ${ }^{[27]}$. 


\section{Weaknesses}

There were some limitations to this study. First, our search was developed to find papers that evaluated measurement properties of PROMs used in gout. As a result, we may have missed PROMs used in gout for which no evaluation of the psychometric properties are yet available. For instance, several new generic item banks, for example, those developed for the Patient-Reported Outcomes Measurement Information System project, were not included in this review for that reason ${ }^{[47]}$. Evaluation of measurement properties of such measures in gout seems very relevant. Moreover, no ICF core set for gout is currently available. The comparative ICF core set we used consisted of the ICF core set of acute inflammatory arthritis and a preliminary ICF core set derived in a recent study in which a core set of gout ICF categories considered relevant by a panel of experts physicians was defined ${ }^{[28,29]}$. The results regarding content validity should therefore be considered preliminary and interpreted with some caution. Another limitation to the evaluation of the content of the PROMs is that all health concepts related to emotional functioning (e.g., "Have you been very nervous?") were linked to a single category, namely 'b152 emotional functions'. Since health concepts relating to emotional functioning were the second most popular category in the included PROMs, and represented quite diverse emotional experiences, different PROMs could probably be characterized in more detail with respect to the various aspects of emotional functioning they assess. Finally, authors of the included papers were usually insufficiently clear about whether patients had active gouty arthritis, or were studied in the so-called inter-critical periods of the disease. Properties of the included PROMs are likely to differ between these subpopulations, which limits the generalizability of our results. For future studies we recommend that authors provide information on the percentage of patients with active arthritis included in the study.

\section{Discussion on findings}

The results of this study show that various PROMs are available for gout, covering the majority of the outcome domains that have been endorsed by OMERACT for use in clinical studies in this field. Interesting was the absence of studies assessing the properties of PROMs for the OMERACT key outcomes of 'joint swelling' and 'joint tenderness'. Possibly because in many gout clinical studies these outcomes are not applied as a PROM, but are rather assessed by the physician ${ }^{[48,49]}$. Nevertheless, patientreports of these domains have been done in gout clinical studies, so that evaluation of their measurement properties is desired ${ }^{[50,51]}$. Also, no studies were found examining the measurement properties of instruments that can be used to derive health utilities for health-economic studies. 
Only the physical functioning subscale of the SF-36v2 was rated favorably for all measurement properties in this systematic review. Moreover, in one of the included studies, a direct comparison with the HAQ-DI and HAQ-II showed that it was the only instrument without floor and ceiling effects, suggesting it better targets the disability levels of gout patients ${ }^{[37]}$. Therefore, current evidence suggests that the SF-36 physical functioning subscale can be recommended for assessing disability in gout. In measuring disability, the HAQ-DI was the only other instrument for which sufficient studies of high quality were available to provide a comprehensive evaluation of its measurement properties. However, this instrument scored inconclusively for construct validity, and floor and ceiling effects. Based on the current evidence, both the VAS and the SF-36v2 bodily pain subscale may be recommended for measuring pain, as almost all measurement properties were supported by high quality studies. However, in general, few studies have yet assessed the psychometric properties of single-item pain measures.

Of the gout-specific PROMs, the health status measuring GAQ2.0 was most extensively evaluated in the literature. Although its subscales showed no floor and ceiling effects, and were all rated as positive for content validity, confirming its items contain health concepts relevant for gout populations, the GAQ2.0 does not cover all recommended OMERACT outcome domains (e.g., no activity limitations scale). This potentially limits its usefulness for gout clinical research purposes. Moreover, the available evidence suggests poor reliability and non-responsiveness to change for half of its subscales, and it was one of the few PROMs with a poorer rating for ease of reading. The overall psychometric appraisal of the GAQ2.0 in this systematic review is in line with previously reported concerns regarding this instrument and therefore we suggest caution in use of this PROM ${ }^{[17]}$. For assessing health-related quality of life, the current evidence suggests the SF-36v2 may be used as an alternative.

For other instruments, no strong conclusions regarding their psychometric quality were possible, despite the availability of at least one study of most measurement properties for each instrument. With respect to construct validity, this was mostly because authors failed to specify hypotheses about the associations they expected to find. Construct validation is an iterative process in which confidence in the degree to which a PROM actually reflects the construct it intends to measure increases as applications of the measure consistently yield results that would be expected, given theories about how this construct relates to other constructs ${ }^{[52]}$. Therefore, especially for newly introduced PROMs, proper evaluation of construct validity requires researchers to be specific about expected relations among instruments included in the assessment; taking into account that the relations between the substantive constructs, measurement error and 
method of measurement all contribute to the observed relations between instruments. For instance, PROMs can be expected to have relatively high intercorrelations, and therefore only limited information about construct validity can be extracted from the finding that significant correlations exist between a number of PROMs. Neither is it the case that higher correlations are always indicative of greater construct validity. Assessments of test-retest reliability in acute gout are complicated by the often rapid improvement that occurs, even without treatment, in the clinical status of patients. This makes it challenging to select a population of stable patients, which led to the many indeterminate ratings in this review. Therefore, for multi-item PROMs, reliability should, in our opinion, be assessed using coefficients that can be calculated from the interitem covariance matrix, such as Cronbach's alpha.

\section{Implications for practice}

For clinicians working in the field of gout, it may be necessary to understand that little evidence is currently available on the measurement properties of commonly used PROMs, and more importantly, which consequences this may have on outcomes data when poorly supported PROMs are used. In particular as some of the PROMs, for instance the single-item pain PROMs, may be used in daily practice for determining the severity of the pain associated with a gout flare. However, also because evidence from clinical trials, where PROMs are commonly used to collect data, are generally used for developing gout guidelines or management recommendations for in daily clinical practice.

\section{Implications for research}

To ensure high-quality patient-reported outcomes data is collected in gout research it is essential that valid and reliable PROMs are used. Their usage may enhance the feasibility of studies by, for example, creating less measurement error, leading to a smaller required sample size. However, the results from this study show that the measurement properties of the PROMs commonly used in gout clinical research settings are weakly supported. To enhance their position in gout research, we recommend that more evidence on the validity and reliability of PROMs used in gout becomes available. Choosing the most suitable PROM from other alternatives may therefore become easier, and endorsing PROMs for measuring relevant gout outcomes in clinical research, as done by OMERACT, will ideally be based on solid evidence supporting the measurement properties of PROMs.

\section{Conclusions}

In conclusion, the present report presents the results of an evaluation of the content and literature supporting the measurement properties of commonly used PROMs in gout. 
The results suggest that PROMs are available to assess the majority of the recommended OMERACT core outcome domains for use in clinical research for acute and chronic gout. However, the SF-36 physical functioning subscale is the only PROM that currently meets all the quality criteria we imposed for this review. Many of the commonly used PROMs in this field are currently not yet well supported and more studies on their measurement properties are needed among both acute and chronic gout populations.

\section{Acknowledgements}

Access and permission for use of the Gout Assessment Questionnaires 2.0 was granted by Takeda Pharmaceuticals, who has ownership and copyright of this instrument. The University of Leeds, owner and copyright owner of the Rheumatoid Arthritis-Work Instability Scale, granted access and permission to use this questionnaire. 


\section{REFERENCES}

1. Richette P, Bardin T. Gout. Lancet. 2010;375:318-28.

2. Edwards NL, Sundy JS, Forsythe A, Blume S, Pan F, Becker MA. Work productivity loss due to flares in patients with chronic gout refractory to conventional therapy. J Med Econ. 2011;14(1):10-5.

3. Becker MA, Schumacher HR, Benjamin KL, Gorevic P, Greenwald M, Fessel J, et al. Quality of life and disability in patients with treatment-failure gout. J Rheumatol. 2009;36(5):1041-8.

4. Terkeltaub RA, Schumacher HR, Carter JD, Baraf HSB, Evans RR, Wang J, et al. Rilonacept in the treatment of acute gouty arthritis: a randomized, controlled clinical trial using indomethacin as the active comparator. Arthritis Res Ther. 2013;15:R25.

5. Chandratre P, Roddy E, Clarson L, Richardson J, Hider SL, Mallen CD. Health-related quality of life in gout: a systematic review. Rheumatology. 2013;52(11):2031-40.

6. Mokkink LB, Terwee CB, Stratford PW, Alonso J, Patrick DL, Riphagen I, et al. Evaluation of the methodological quality of systematic reviews of health status measurement instruments. Qual Life Res. 2009;18(3):313-33.

7. Schumacher HR, Edwards LN, Perez-Ruiz F, Becker M, Chen LX, Furst DE, et al. Outcome measures for acute and chronic gout. J Rheumatol. 2005;32(12):2452-5.

8. Schumacher HR, Taylor W, Joseph-Ridge N, Perez-Ruiz F, Chen LX, Schlesinger N, et al. Outcome evaluations in gout. J Rheumatol. 2007;34(6):1381-5.

9. Taylor WJ, Schumacher Jr HR, Baraf HSB, Chapman P, Stamp L, Doherty M, et al. A modified Delphi exercise to determine the extent of consensus with OMERACT outcome domains for studies of acute and chronic gout. Ann Rheum Dis. 2008;67:888-91.

10. Schumacher HR, Taylor W, Edwards L, Grainger R, Schlesinger N, Dalbeth N, et al. Outcome domains for studies of acute and chronic gout. J Rheumatol. 2009;36(10):2342-5.

11. Boers M, Brooks P, Strand CV, Tugwell P. The OMERACT filter for Outcome Measures in Rheumatology. J Rheumatol. 1998;25(2):198-9.

12. Grainger R, Taylor WJ, Dalbeth N, Perez-Ruiz F, Singh JA, Waltrip RW, et al. Progress in measurement instruments for acute and chronic gout studies. J Rheumatol. 2009:36(10):2346-55.

13. Singh JA, Taylor WJ, Dalbeth N, Simon LS, Sundy J, Grainger R, et al. OMERACT endorsement of measures of outcome for studies of acute gout. J Rheumatol. 2014;41(3):569-73.

14. Dalbeth N, Zhong CS, Grainger R, Khanna D, Khanna PP, Singh JA, et al. Outcome measures in acute gout: A systematic literature review. J Rheumatol. 2014;41(3):558-68.

15. Taylor WJ, Redden D, Dalbeth N, Schumacher HR, Edwards NL, Simon LS, et al. Application of the OMERACT filter to measures of core outcome domains in recent clinical studies of acute gout. J Rheumatol. 2014; $41(3): 574-80$.

16. Taylor WJ, Schumacher HR, Singh JA, Grainger R, Dalbeth N. Assessment of outcome in clinical trials of gout--a review of current measures. Rheumatology (Oxford). 2007;46(12):1751-6.

17. Singh JA, Taylor WJ, Simon LS, Khanna PP, Stamp LK, McQueen FM, et al. Patient-reported Outcomes in Chronic Gout: A Report from OMERACT 10. J Rheumatol. 2011;38(7):1452-7. 
18. Terwee CB, Jansma EP, Riphagen II, De Vet HCW. Development of a methodological PubMed search filter for finding studies on measurement properties of measurement instruments. Qual Life Res. 2009;18(8):1115-23.

19. Moher D, Liberati A, Tetzlaff J, Altman D, The PRISMA Group. Preferred Reporting Items for Systematic Reviews and Meta-Analyses: The PRISMA Statement. PloS Med. 2009;6(7):e1000097.

20. Reeve BB, Wyrwich KW, Wu AW, Velikova G, Terwee CB, Snyder CF, et al. ISOQOL recommends minimum standards for patient-reported outcome measures used in patient-centered outcomes and comparative effectiveness research. Qual Life Res. 2013;22(8):1889-905.

21. Mokkink LB, Terwee CB, Patrick DL, Alonso J, Stratford PW, Knol DL, et al. The COSMIN checklist for assessing the methodological quality of studies on measurement properties of health status measurement instruments: An international Delphi study. Qual Life Res. 2010;19(4):539-49.

22. Angst F. The new COSMIN guidelines confront traditional concepts of responsiveness. BMC Med Res Methodol. 2011;11:152.

23. Sijtsma K. On the use, the misuse, and the very limited usefulness of cronbach's alpha. Psychometrika. 2009;74(1):107-20.

24. van der Ark LA, van der Palm DW, Sijtsma K. A Latent Class Approach to Estimating Test-Score Reliability. Appl Psychol Meas. 2011;35(5):380-92.

25. Revicki D, Hays RD, Cella D, Sloan J. Recommended methods for determining responsiveness and minimally important differences for patient-reported outcomes. J Clin Epidemiol. 2008;61(2):102-9.

26. Terwee CB, Bot SDM, de Boer MR, van der Windt DAWM, Knol DL, Dekker J, et al. Quality criteria were proposed for measurement properties of health status questionnaires. J Clin Epidemiol. 2007;60(1):34-42.

27. Cieza A, Fayed N, Bickenbach J, Prodinger B. Refinements of the ICF Linking Rules to strengthen their potential for establishing comparability of health information. Disabil Rehabil. 2016;1-10.

28. Grill E, Zochling J, Stucki G, Mittrach R, Scheuringer M, Liman W, et al. International Classification of Functioning, Disability and Health (ICF) Core Set for patients with acute arthritis. Clin Exp Rheumatol. 2007; $25: 252-8$.

29. Kool EM, Nijsten MJ, van Ede AE, Jansen TL, Taylor WJ. Discrepancies in how the impact of gout is assessed in outcomes research compared to how health professionals view the impact of gout, using the lens of the International Classification of Functioning, Health and Disability (ICF). Clin Rheumatol. 2016;35(9):2259-68.

30. Hambleton RK, Swaminathan H, Rogers HJ. Fundamentals of item response theory. Newbury Park, California: Sage Publications, Inc.; 1991.

31. Choi S, Cook K, Dodd B. Parameter recovery for the partial credit model using MULTILOG. J Outcome Meas. 1997;1(2):114-42.

32. Ten Klooster PM, Vonkeman HE, Oude Voshaar MAH, Bode C, Van De Laar MAFJ. Experiences of gout-related disability from the patients' perspective: A mixed methods study. Clin Rheumatol. 2014;33(8):1145-54.

33. Álvarez-Hernández E, Zamudio-Lerma JA, Burgos-Martínez G, Álvarez-Etchegaray SE, Peláez-Ballestas I, Vázquez-Mellado J. [Measurement of health-related quality of life and functional capacity in patients with chronic tophaceous gout]. Reumatol Clin. 2009;5(3):103-8.

34. Hirsch JD, Lee SJ, Terkeltaub R, Khanna D, Singh JA, Sarkin A, et al. Evaluation of an instrument assessing 
influence of Gout on health-related quality of life. J Rheumatol. 2008;35(12):2406-14.

35. Singh JA, Yang S, Strand V, Simon L, Forsythe A, Hamburger S, et al. Validation of pain and patient global scales in chronic gout: data from two randomised controlled trials. Ann Rheum Dis. 2011;70(7):1277-81.

36. Taylor WJ, House M, Horne A, McQueen FM, Dalbeth N. The work instability scale predicts absenteeism in people with gout and suggests a higher risk for those in manual occupations. J Clin Rheumatol. 2012;18(8):405-10.

37. ten Klooster PM, Oude Voshaar MAH, Taal E, van de Laar MAFJ. Comparison of measures of functional disability in patients with gout. Rheumatology. 2011;50(4):709-13.

38. Van Groen MM, Ten Klooster PM, Taal E, Van De Laar MAFJ, Glas CAW. Application of the health assessment questionnaire disability index to various rheumatic diseases. Qual Life Res. 2010;19(9):1255-63.

39. Taylor WJ, Colvine K, Gregory K, Collis J, McQueen FM, Dalbeth N. The Health Assessment Questionnaire Disability Index is a valid measure of physical function in gout. Clin Exp Rheumatol. 2008;26(4):620-6.

40. Álvarez-Hernández E, Peláez-Ballestas I, Vázquez-Mellado J, Terán-Estrada L, Bernard-Medina AG, Espinoza J, et al. Validation of the Health Assessment Questionnaire disability index in patients with gout. Arthritis Rheum. 2008 May 15;59(5):665-9.

41. Wallace B, Khanna D, Aquino-Beaton C, Singh JA, Duffy E, Elashoff D, et al. Performance of Gout Impact Scale in a longitudinal observational study of patients with gout. Rheumatology. 2016;55(6):982-90.

42. Aati O, Taylor WJ, Siegert RJ, Horne A, House ME, Tan P, et al. Development of a patient-reported outcome measure of tophus burden: the Tophus Impact Questionnaire (TIQ-20). Ann Rheum Dis. 2015;74(12):2144-50.

43. Spaetgens B, Van der linden S, Boonen A. The gout assessment questionnaire 2.0: Cross-cultural translation into dutch, aspects of validity and linking to the international classification of functioning, disability and health. Rheumatology. 2014;53(4):678-85.

44. Khanna PP, Perez-Ruiz F, Maranian P, Khanna D. Long-term therapy for chronic gout results in clinically important improvements in the health-related quality of life: Short form-36 is responsive to change in chronic gout. Rheumatology. 2011;50(4):740-5.

45. Boers M, Jacobs JWG, van Vliet Vlieland TPM, van Riel PLCM. Consensus Dutch health assessment questionnaire. Ann Rheum Dis. 2007;66:132-3.

46. Reilly M, Zbrozek A, Dukes E. The validity and reproducibility of a work productivity and activity impairment instrument. Pharmacoeconomics. 1993;4(5):353-65.

47. Cella D, Riley W, Stone A, Rothrock N, Reeve B, Yount S, et al. The patient-reported outcomes measurement information system (PROMIS) developed and tested its first wave of adult self-reported health outcome item banks: 2005-2008. J Clin Epidemiol. 2010;63(11):1179-94.

48. Schlesinger N, De Meulemeester M, Pikhlak A, Yücel AE, Richard D, Murphy V, et al. Canakinumab relieves symptoms of acute flares and improves health-related quality of life in patients with difficult-to-treat Gouty Arthritis by suppressing inflammation: results of a randomized, dose-ranging study. Arthritis Res Ther. 2011;13(2):R53.

49. Xu L, Liu S, Guan M, Xue Y. Comparison of Prednisolone, Etoricoxib, and Indomethacin in Treatment of Acute Gouty Arthritis: An Open-Label, Randomized, Controlled Trial. Med Sci Monit. 2016;22:810-7. 
50. Schumacher HR, Evans RR, Saag KG, Clower J, Jennings W, Weinstein SP, et al. Rilonacept (interleukin-1 trap) for prevention of gout flares during initiation of uric acid-lowering therapy: Results from a phase III randomized, double-blind, placebo-controlled, confirmatory efficacy study. Arthritis Care Res. 2012;64(10):1462-70.

51. Dalbeth N, Jones G, Terkeltaub R, Khanna D, Kopicko J, Bhakta N, et al. Lesinurad, A Selective Uric Acid Reabsorption Inhibitor, in Combination with Febuxostat in Patients with Tophaceous Gout: Findings of a Phase III Clinical Trial. Arthritis Rheumatol. 2017;69(9):1903-13.

52. Strauss ME, Smith GT. Construct validity: Advances in Theory and Methodology. Annu Rev Clin Psychol. $2009 ; 5: 1-25$. 


\section{SUPPLEMENTAL MATERIAL}

Supplemental material 1: Search strings applied in both the Pubmed and EMBASE databases for finding appropriate literature.

Initial search string Pubmed:

((gout[MeSH Terms]) OR (gout[Title/Abstract] OR gouty[Title/Abstract])) AND ((valid*[Title/Abstract] OR reliab*[Title/Abstract] OR responsiveness[Title/Abstract] OR discriminative[Title/Abstract] OR Rasch[Title/Abstract] OR "item response theory"[Title/ Abstract] OR "sensitivity to change"[Title/Abstract] OR "measurement error"[Title/ Abstract]))

Initial search string Embase:

gout:ab,ti OR gouty:ab,ti AND valid*:ab,ti OR reliab*:ab,ti OR responsiveness:ab,ti OR discriminative:ab,ti OR Rasch:ab,ti OR "item response theory":ab,ti OR "sensitivity to change":ab,ti OR "measurement error":ab,ti 
Supplemental material 2: Linking of instrument subscales to item perspectives and categorization of response options, according to the 2016 ICF linking rules (Cieza et al. 2016).

\begin{tabular}{|c|c|c|c|}
\hline \multirow[b]{2}{*}{ Instrument } & \multirow[b]{2}{*}{ Subscale } & \multicolumn{2}{|c|}{ ICF Linking 2016} \\
\hline & & Item Perspective $^{a}$ & Response category ${ }^{b}$ \\
\hline \multirow[t]{8}{*}{$S F-36 v 2$} & Physical functioning & $\mathrm{DC}$ & IN \\
\hline & Role-physical & $\mathrm{DP}$ & $\mathrm{F}$ \\
\hline & Bodily pain & $\mathrm{D}$ & IN \\
\hline & General health & APP & IN, CoA \\
\hline & Vitality & $\mathrm{DP}$ & $\mathrm{F}$ \\
\hline & Social functioning & $\mathrm{DP}$ & IN, F \\
\hline & Role-emotional & DP & $\mathrm{F}$ \\
\hline & Mental health & DP & $\mathrm{F}$ \\
\hline \multirow[t]{6}{*}{ MOS-20 } & Physical function & $\mathrm{DC}$ & $\mathrm{F}$ \\
\hline & Role functional & $D P, D C$ & $\mathrm{~F}$ \\
\hline & Social functioning & DP & $\mathrm{F}$ \\
\hline & Mental health & $\mathrm{DP}$ & $\mathrm{F}$ \\
\hline & Current perception of health & APP & IN, CoA \\
\hline & Pain & $\mathrm{D}$ & IN \\
\hline \multirow[t]{9}{*}{ AIMS } & Mobility & $D P, D C, N o D$ & $\mathrm{CoA}$ \\
\hline & Physical activity & DC, NoD & $\mathrm{COA}$ \\
\hline & Dexterity & DC & $\mathrm{COA}$ \\
\hline & Household activity & DC, NoD & $\mathrm{COA}$ \\
\hline & Social activities & DP & $\mathrm{F}$ \\
\hline & Activities of daily living & DC, NoD & $\mathrm{COA}$ \\
\hline & Pain & $\mathrm{D}$ & IN, F, DU \\
\hline & Depression & $\mathrm{DP}$ & $\mathrm{F}$ \\
\hline & Anxiety & $\mathrm{DP}$ & $\mathrm{F}$ \\
\hline \multirow[t]{5}{*}{ GAQ 2.0} & Gout concern overall & DP & IN \\
\hline & Gout medication side effects & $\mathrm{DP}$ & IN \\
\hline & Unmet gout treatment need & APP & IN \\
\hline & Well-being during attack & $\mathrm{DP}, \mathrm{DC}$ & IN, F \\
\hline & Gout concern during attack & $\mathrm{DP}$ & IN \\
\hline $\mathrm{HAQ}-\mathrm{DI}$ & - & DC, NoD & IN, QA \\
\hline$H A Q-\|$ & - & DC & IN \\
\hline TIQ-20 & - & D, DP & $\mathrm{COA}$ \\
\hline RA-WI & - & D, DP & $\mathrm{CoA}$ \\
\hline VAS pain & - & $\mathrm{D}$ & IN \\
\hline Likert pain & - & $\mathrm{D}$ & IN \\
\hline NRS pain & - & $\mathrm{D}$ & IN \\
\hline VAS PGA & - & APP & IN \\
\hline Physical function NRS & - & $\mathrm{DP}, \mathrm{DC}$ & IN \\
\hline
\end{tabular}


$(\mathrm{COA})$, qualitative attributes $(\mathrm{QA})$

ICF, International Classification of Functioning, disability and Health; SF-36v2, Short Form-36 item version 2; MOS-20, Medical Outcomes Study 20-item Short Form Health Survey; AIMS, Arthritis Impact Measurement Scales; GAQ 2.0, Gout Assessment Questionnaire 2.0; HAQ-DI, Health Assessment Questionnaire-Disability Index; HAQII, Health Assessment Questionnaire-II; TIQ-20, 20-item Tophus Impact Questionnaire; RA-WIS, Rheumatoid Arthritis-Work Instability Scale; VAS, Visual Analogue Scale; NRS, Numeric Rating Scale; PGA, patient global assessment; - , not applicable 
Supplemental material 3: Content of the multi- and unidimensional scales used in gout outcome studies according to the International Classification of Functioning (ICF) framework, given as the number, $\mathrm{N}$, and percentage (\%) of total health concepts measured.

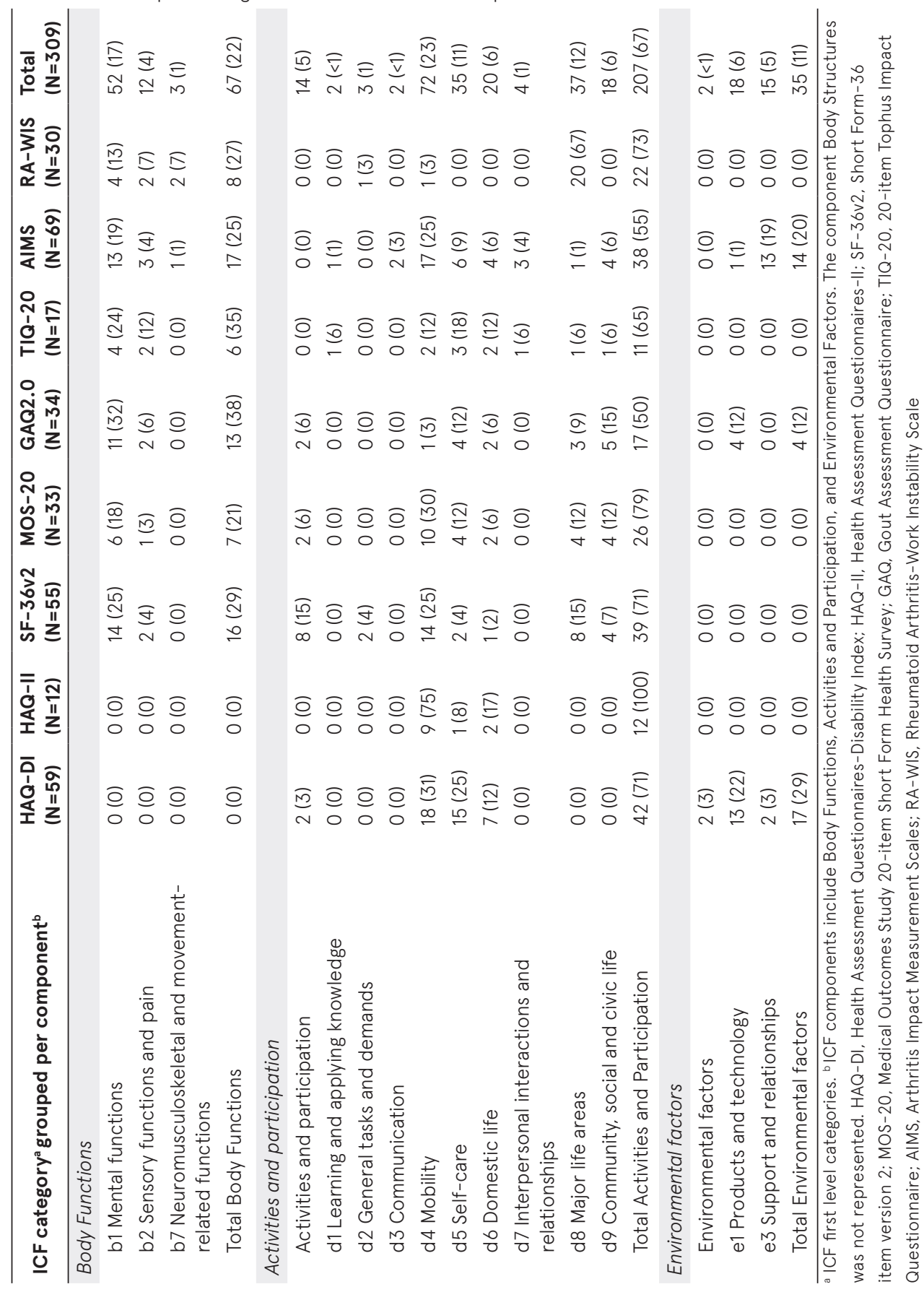





\section{Development and validation of a patient-reported gout attack intensity score for use in gout clinical studies}

Carly A. Janssen, Martijn A.H. Oude Voshaar, Peter M. ten Klooster, Harald E. Vonkeman, Mart A.F.J. van de Laar 


\section{ABSTRACT}

Objective. Inflammation-related symptoms such as pain, swelling and tenderness of the affected joint are frequently assessed using 5-point diary rating scales in gout clinical trials. Combining these into a single gout attack symptom intensity score may be a useful summary measure for these data, which is potentially more responsive to change compared with the individual components. The objective of this study was to develop a patient-reported gout flare intensity score, the Gout Attack Intensity Score (GAIS), for use in clinical studies, that includes components for gout-related pain, swelling and tenderness.

Methods. Data from a randomized controlled trial comparing anakinra to standard of care for the treatment of acute gout attacks were used for this study. A 7-day flare diary was completed by patients, including questions relating to intensity of pain, swelling and tenderness (5-point rating scales). Scalability of these items was assessed using Mokken Scale Analysis, and reliability using greatest lower bound reliability coefficients. Knowngroups validity was evaluated, as well as the responsiveness to change and the presence of floor and ceiling effects.

Results. Scalability of the single items was supported, and GAIS scores were reliable (greatest lower bound $>0.80$ ). GAIS scores demonstrated responsiveness to change with high effect sizes ( $>0.8$ ), and discriminated better between responders and nonresponders compared with its single-item components. No floor and ceiling effects were found.

Conclusion. The GAIS seems to be a reliable and responsive instrument for assessing patient-reported gout attack intensity that may be used in gout clinical studies.

\section{Key Messages.}

- The Gout Attack Intensity Score assesses gout flare symptom intensity in clinical studies.

- The Gout Attack Intensity Score includes components for patient-reported pain, swelling and tenderness.

- The Gout Attack Intensity Score is a reliable, responsive instrument for assessing gout flare intensity. 


\section{INTRODUCTION}

In gout clinical studies, flare diaries are often administered to patients to assess the severity of the various symptoms they may experience as a result of their gout attack ${ }^{[1-4]}$. Such diaries typically include 5-point rating scales that ask patients to rate the intensity of their joint pain, joint swelling and joint tenderness ${ }^{[5]}$. Despite their popularity in study protocols and the endorsement of the individual symptoms as relevant outcome domains by the Outcome Measures in Rheumatology (OMERACT) Gout Working Group, reports of clinical trials in gout rarely present outcomes for symptoms other than pain in the main paper ${ }^{[6,7]}$. Possibly, this is because the different individual symptom measures are considered to provide somewhat redundant information, or because limited evidence is currently available with respect to the validity of these scores ${ }^{[8]}$.

Combining the individual scores of each symptom rating scale included in a gout attack diary (i.e. pain, swelling, tenderness) may summarize the information provided by the individual rating scales into a single score that represents a patient's overall level of gout attack intensity. In addition, combining information from multiple rating scales that assess a single latent variable also serves to reduce the contribution of measurement error to the variability of the scores. This should theoretically increase the ability of the combined score to measure change over time and to discriminate between responders and non-responders, relative to the individual rating scales. This notion is supported by a previous study which already showed that more reliable results can be obtained with composite scores including two to nine pain intensity items, compared with a single 24-h recall pain intensity item, using data from a published randomized controlled clinical trial ${ }^{[9]}$.

In the current study we propose a new three-item Gout Attack Intensity Score, referred to as the GAIS, for use in flare diaries in clinical trials or observational studies in patients with acute gout attacks, and evaluate its measurement properties.

\section{METHODS}

\section{Study database}

Data obtained during an investigator-initiated randomized, double blinded, double dummy, active placebo-controlled non-inferiority trial carried out in The Netherlands across seven rheumatology clinics were used for this study (NTR5234) ${ }^{[10]}$. In short, adult patients with crystal-proven acute gout attacks recruited between February 2016 and 
February 2018 were randomized to treatment with the interleukin-1 inhibitor anakinra, or to treatment with usual care (colchicine, non-steroidal anti-inflammatory drug or corticosteroids) for the treatment of their acute gout attack. Results of this study showed that anakinra was non-inferior to standard of care in the treatment of acute gout flares.

Patients were asked to fill in a 7-day flare diary starting at baseline (day 1), including items about perceived pain [5-point rating scale and 10-point numeric rating scale (NRS)], tenderness (5-point rating scale), swelling (5-point rating scale), treatment response (8-point rating scale) and their global assessment of overall wellbeing [patient global assessment (PGA)] (10-point NRS). For the present study, PGA scores were recoded so that higher scores represent worse wellbeing. A copy of the flare-diary including all its items is included in Supplemental Material 1. At day 1 and day 7, levels of C-reactive protein (CRP) were measured. The study was performed in accordance with the Declaration of Helsinki and was approved by both an independent ethics committee and the institutional review board of each participating centre. All participants provided written informed consent.

For the current study, we used the daily data of the 7-day flare diary of patients in both treatment groups. The GAIS was obtained by taking the mean of the patient-reported 5 -point rating scale pain, rating scale swelling and rating scale tenderness. Since the GAIS contains only three items, only patients who had no missing values for the three single items used to calculate the GAIS were included.

\section{Scaling properties}

Scaling properties were examined using the model of monotone homogeneity (MMH), using the Mokken package in $\mathrm{R} \times 64$ version 3.4.2. The $M M H$ is a non-parametric item response theory model. The model is based on the assumption that there exists a latent variable $(\theta)$ on which a scale's items as well as the persons responding to the items can be ordered. The model can be considered a probabilistic version of polytomous Guttman scaling (PGS). In PGS, each item with $m$ response categories is broken down into $m-1$ item steps, which represent the location on the latent variable where the probability that a person selects the higher of two adjacent response categories jumps from 0 to 1 . Violations of the Guttman model, or Guttman errors, occur if a patient endorses an item step that has a higher location on the latent variable compared with another item step that this patient fails to endorse. In the $\mathrm{MMH}$, the probability that a person will select the higher of two adjacent response categories does not jump from 0 to 1 at a particular location on the latent variable, like in PGS, but is instead described for different values of the latent variable by the item step response functions (ISRF). ISRF are defined as 
$P\left(X_{i} \geq \chi_{i} \mid \theta\right.$, where $X_{i}$ is a random variable that refers to the score on item $i$, which takes on values: $\chi_{i}=0, \ldots m$.

The MMH applies if 1) all $i$ items in a scale measure the same latent variable, i.e. the scale is unidimensional and 2) the ISRF are monotonically non-decreasing throughout the latent variable ${ }^{[11]}$. If the model applies, it supports that higher scores on the scale reflect a higher level of gout attack intensity.

Monotonicity was tested by inspecting plots of the ISRF of each item (e.g. swelling) over the summed score continuum of the two remaining items (e.g. pain and tenderness). Deviations from monotonicity were statistically tested, using group sizes of 5, 10 and 20, with the check.monotonicity function of the Mokken R package. Monotonicity was considered to apply if the plots of ISRF were non-decreasing, the number of statistically significant deviations from monotonicity were zero and the magnitude of the violations, as indicated by the crit statistic (critical value for model violations statistic) was $\leq 40{ }^{[12]}$. Unidimensionality was tested using Loevinger's scalability coefficients, which take on lower values as the number of Gutmann errors increase. Both item-level scalability (Hi), as well as scale-level scalability coefficients $(H)$ were assessed. Scale-level scalability coefficients between 0.30 and $<0.40$ suggest weak scalability, between 0.40 and $<0.50$ suggest a moderate scale and $\geq 0.5$ suggests a strong scale ${ }^{[13,14]}$. A lower bound of 0.3 was considered the cut-off point for item-level scalability, according to a rule of thumb ${ }^{[15]}$. Data from each day of the gout flare diary were used for the analyses.

\section{Reliability}

Reliability of the GAIS observed on days 1-7 was assessed using greatest lower bound (GLB) coefficients, since this statistic has been shown to yield a more realistic estimate of the lower bound value for test reliability compared with the more frequently applied Cronbach's alpha ${ }^{[16]}$. For tests with small numbers of items, such as in our study, GLB has been shown to be relatively unaffected by sampling bias, even with small sample sizes ${ }^{[17]}$. Reliability coefficients of 0.80 were considered to be satisfactory for use in clinical research settings ${ }^{[18]}$. The Psych and Rcsdp package of the statistical programme $\mathrm{R} \times 64$ version 3.4 .2 and Rstudio were used for the reliability analyses. The inter-item covariance matrix was obtained in SPSS Version 22.

\section{Validity}

Known-groups validity was examined by determining whether groups with different levels of self-reported treatment response could be distinguished based on their scores on the GAIS, joint swelling, joint tenderness, pain and PGA, at baseline. For this analysis, we partitioned 
the total sample in two groups based on their level of self-reported treatment response: patients who scored within the range of 1 (completely resolved) to 4 (somewhat improved) were considered responders, and patients who scored in the range 5 (unchanged) to 8 (very much worse) were considered non-responders. Score differences between responders and non-responders were compared using one-way analysis of variance in SPSS, version 22. It was expected that patients who reported having a poorer treatment response (nonresponders) would have significantly higher scores on all the instruments compared with the responders. To compare the discriminative ability between different instruments, the relative efficiency (RE) of each instrument was compared with that of the GAIS ${ }^{[19]}$. It was hypothesized that the discriminative ability of the GAIS would be greater than that of the single items of which it consists, represented by a higher RE.

\section{Responsiveness and sensitivity to change}

Responsiveness to change was compared between different instruments by calculating Cohen's $d$ effect sizes (ES) as [mean day 1 - mean $t$ days]/pooled standard deviation (SD). In the analyses, only cases for whom on both day 1 and day 5 (or day 1 and day 7 for CRP) data were available for each instrument were included. In calculating the ES for CRP, log-transformed data were used. An ES of 0.2 was considered a small effect, 0.5 a moderate effect and 0.8 a large effect ${ }^{[20]}$. Since anti-inflammatory treatment at recommended dosages was administered to all patients, and gout attacks are generally known to have a self-limiting course of limited duration, we expected to observe large improvements in clinical status over time, and thus large effect sizes for all instruments. Analyses were done using SPSS, version 22, and Microsoft Excel.

\section{Floor and ceiling effects}

Floor (ceiling) effects, defined as the proportions of patients scoring the worst (best) possible score were compared between the GAIS and the individual component scores, the NRS pain and the NRS PGA of the gout flare diary, at baseline. We hypothesized that ceiling effects (lowest possible score) would be absent for all instruments, considering that at baseline, gout attack symptoms would be expected to be at their worst. Floor or ceiling effects were considered to be problematic in case $>15 \%$ of patients scored the worst or best possible score, respectively ${ }^{[21]}$. Analyses were done using SPSS, version 22.

\section{RESULTS}

\section{Baseline population}

A total of 88 patients were enrolled in the study. The baseline population consisted 
predominantly of middle-aged men suffering from mono-articular gout. Other baseline characteristics of the entire sample are listed in Table 1. At baseline, 76 (86\%) patients completed the gout flare diary. For the following days, diaries for which the summed score could be calculated were available for $82,85,83,86,79$ and 82 patients on days $2-7$, respectively.

Table 1: Baseline characteristics

\begin{tabular}{|c|c|c|c|}
\hline Characteristic & $\begin{array}{l}\text { Score range } \\
\text { of measure }\end{array}$ & Value & $\mathbf{N}$ \\
\hline Age, years, mean \pm SD & - & $61.6 \pm 12.8$ & 88 \\
\hline Male sex, n (\%) & - & $83(94.3)$ & 88 \\
\hline $\mathrm{BMI}, \mathrm{kg} / \mathrm{m}^{2}$, mean $\pm \mathrm{SD}$ & - & $29.1 \pm 4.1$ & 86 \\
\hline Systolic blood pressure, $\mathrm{mm} \mathrm{Hg}$, mean \pm SD & $0-999$ & $143.3 \pm 22.9$ & 79 \\
\hline Diastolic blood pressure $\mathrm{mm} \mathrm{Hg}$, mean \pm SD & $0-999$ & $85.2 \pm 14.5$ & 80 \\
\hline SUA, mmol/L, median (Q1, Q3) & $0-9$ & $0.51(0.44,0.59)$ & 82 \\
\hline CRP, mg/L, median (Q1, Q3) & 0-999 & $15.0(6.0,32.0)$ & 82 \\
\hline GAIS, median (Q1, Q3) & $1-5$ & $3.67(3,4)$ & 76 \\
\hline 5-point rating scale pain, median (Q1, Q3) & $1-5$ & $4(3,4)$ & 76 \\
\hline 5-point rating scale tenderness, median (Q1, Q3) & $1-5$ & $4(3,4)$ & 76 \\
\hline 5-point rating scale swelling, median (Q1, Q3) & $1-5$ & $3(3,4)$ & 76 \\
\hline NRS pain, mean \pm SD & $0-10$ & $6.47 \pm 1.70$ & 76 \\
\hline $\begin{array}{l}\text { Number of gout attacks in previous } \leq 12 \text { months, median } \\
\text { (Q1, Q3) }\end{array}$ & $0-999$ & $3.0(1.0,4.0)$ & 88 \\
\hline Intermittent gout, n (\%) & - & $71(80.7)$ & 88 \\
\hline \multicolumn{4}{|l|}{ Gout classification ${ }^{b}, n(\%)$} \\
\hline Monoarticular & - & $53(60.2)$ & 88 \\
\hline Oligoarticular & - & $28(31.8)$ & 88 \\
\hline Polyarticular & - & $7(8.0)$ & 88 \\
\hline \multicolumn{4}{|l|}{ Comorbidities, $n(\%)$} \\
\hline Diabetes Mellitus & - & $8(15.1)$ & 53 \\
\hline Hypertension & - & $27(50.9)$ & 53 \\
\hline Cardiovascular disease & - & $29(54.7)$ & 53 \\
\hline Renal disorders & - & $5(9.4)$ & 53 \\
\hline Musculoskeletal disease ${ }^{c}$ & - & $10(18.9)$ & 53 \\
\hline Gastrointestinal disorders & - & $10(18.9)$ & 53 \\
\hline Neurological disorders & - & $4(7.5)$ & 53 \\
\hline
\end{tabular}

SD, standard deviation; BMI, body mass index; SUA, serum urate acid; CRP, C-reactive protein; GAIS, gout attack intensity score; NRS, numeric rating scale; N, total number of patients; Q1/Q3, first and third quartile respectively. ${ }^{a}$ Compared to patients having chronic gout.

${ }^{\mathrm{b}}$ Monoarticular implies one joint has been affected by gout; oligoarticular $>1$ but $<5$ joints have been affected by gout; polyarticular $\geq 5$ joints have been affected by gout.

${ }^{c}$ Diseases other than gout. 


\section{Scalability}

The item-level scalability coefficients $(\mathrm{Hi})$ for pain, tenderness and swelling were all greater than the lower bound cut-off value of 0.3 for days 1 through 7 , ranging from $0.61-$ $0.83,0.63-0.84$ and $0.44-0.75$, respectively (Table 2). Scale-level scalability coefficients for the GAIS revealed strong unidimensionality $(H \geq 0.50)$, with $H$ coefficients ranging from $0.56-0.80$ across the seven days. Statistical testing of monotonicity showed no significant deviations from zero at group sizes 5, 10 and 20 for days 1 through 7, as well as no critical values $>40$. These results support that the ISRFs and the expected mean items score were monotonically increasing over the latent variable (Table 2 and Supplemental Material 2). This was also confirmed by visual inspection of the ISRF plots. Overall, the results of the Mokken scaling analysis support the conclusion that all items relate to the same latent variable, and that higher scores on GAIS indicate a higher level of gout attack intensity.

\section{Reliability}

The median reliability coefficient for days $1-7$ was 0.84 , with GLB coefficients ranging between 0.78 and 0.91 . Only one GLB was $<0.80$. These results suggest that scores on the GAIS are generally sufficiently reliable for use in clinical trials (Table 2).

Table 2: Scalability and reliability coefficients, of the single-component items and GAIS

\begin{tabular}{|c|c|c|c|c|c|c|c|c|c|}
\hline \multirow[b]{3}{*}{ Day } & \multirow[b]{3}{*}{$\mathrm{N}$} & \multicolumn{4}{|c|}{ Unidimensionality, $\boldsymbol{H}$ (SE) } & \multicolumn{3}{|c|}{ Monotonicity ${ }^{a}$} & \multirow{3}{*}{ Reliability } \\
\hline & & \multicolumn{3}{|c|}{ Item level } & \multirow{2}{*}{$\begin{array}{l}\text { Scale level } \\
\text { GAIS }\end{array}$} & \multirow[b]{2}{*}{ Pain } & \multirow[b]{2}{*}{ Tenderness } & \multirow[b]{2}{*}{ Swelling } & \\
\hline & & Pain & Tenderness & Swelling & & & & & \\
\hline 1 & 76 & $\begin{array}{l}0.61 \\
(0.07)\end{array}$ & $0.63(0.07)$ & $0.44(0.10)$ & $0.56(0.07)$ & $0(0)$ & $0(28)$ & $0(17)$ & 0.78 \\
\hline 2 & 82 & $\begin{array}{l}0.67 \\
(0.06)\end{array}$ & $0.73(0.05)$ & $0.62(0.07)$ & $0.68(0.06)$ & $0(0)$ & $0(0)$ & $0(0)$ & 0.83 \\
\hline 3 & 85 & $\begin{array}{l}0.66 \\
(0.08)\end{array}$ & $0.72(0.06)$ & $0.59(0.09)$ & $0.66(0.07)$ & $0(0)$ & $0(0)$ & $0(0)$ & 0.80 \\
\hline 4 & 83 & $\begin{array}{l}0.71 \\
(0.07)\end{array}$ & $0.69(0.06)$ & $0.64(0.07)$ & $0.68(0.06)$ & $0(0)$ & $0(0)$ & $0(0)$ & 0.84 \\
\hline 5 & 86 & $\begin{array}{l}0.83 \\
(0.05)\end{array}$ & $0.81(0.05)$ & $0.75(0.07)$ & $0.80(0.05)$ & $0(-2)$ & $0(0)$ & $0(0)$ & 0.89 \\
\hline 6 & 79 & $\begin{array}{l}0.78 \\
(0.05)\end{array}$ & $0.76(0.05)$ & $0.70(0.07)$ & $0.75(0.05)$ & $0(0)$ & $0(0)$ & $0(0)$ & 0.88 \\
\hline 7 & 82 & $\begin{array}{l}0.83 \\
(0.05)\end{array}$ & $0.84(0.04)$ & $0.72(0.07)$ & $0.80(0.05)$ & $0(0)$ & $0(0)$ & $0(0)$ & 0.91 \\
\hline
\end{tabular}

a Given as the number of statistical significant deviations from monotonicity (critical value for violations), at group size $=10$. ${ }^{b}$ Reliability coefficient, $r$, according to the greatest lower bound. $H$ : scalability coefficient for item-level scalability $(H i)$, and scale-level scalability $(H)$; SE, standard error; N, sample size; GAIS, gout attack intensity score 


\section{Validity}

All instruments, with the exception of the NRS PGA, were able to significantly $(p<0.05)$ discriminate between the two groups of self-reported treatment response, showing mean scores in the non-responders group that were higher than the responders for all instruments, as hypothesized (Table 3 ). As expected, the RE of the single items pain (0.41), swelling (0.81) and tenderness (0.66) were all lower than the GAIS (1.00), implying the GAIS had a greater discriminative ability. Only the NRS pain had a higher RE than the GAIS, which makes this instrument a more suitable instrument for discriminating between the groups when solely measuring pain.

Table 3: Ability of instruments to discriminate between difference groups of self-reported treatment response

\begin{tabular}{|c|c|c|c|c|}
\hline \multirow[b]{2}{*}{ Instrument } & \multicolumn{2}{|c|}{ Groups of treatment response on day 1} & \multirow[b]{2}{*}{$F$} & \multirow[b]{2}{*}{ RE } \\
\hline & $\begin{array}{c}\text { Non-responders }(\mathrm{N}=44) \\
\text { mean } \pm \mathrm{SD}\end{array}$ & $\begin{array}{c}\text { Responders }(\mathrm{N}=27) \\
\text { mean } \pm \mathrm{SD}\end{array}$ & & \\
\hline GAIS & $3.69 \pm 0.64$ & $3.10 \pm 0.66$ & $13.92^{*}$ & 1.00 \\
\hline Joint pain ${ }^{a}$ & $3.66 \pm 0.78$ & $3.22 \pm 0.70$ & $5.72^{*}$ & 0.41 \\
\hline Joint tenderness ${ }^{a}$ & $3.80 \pm 0.85$ & $3.15 \pm 0.91$ & $9.20^{*}$ & 0.66 \\
\hline Joint swellinga & $3.61 \pm 0.87$ & $2.93 \pm 0.78$ & $11.31^{*}$ & 0.81 \\
\hline NRS joint pain ${ }^{b}$ & $7.11 \pm 1.38$ & $5.56 \pm 1.55$ & $19.32^{*}$ & 1.39 \\
\hline NRS PGA ${ }^{b}$ & $5.68 \pm 2.09$ & $4.85 \pm 1.35$ & 3.39 & 0.24 \\
\hline
\end{tabular}

a Measured on a 5 -point rating scale. ${ }^{\mathrm{b}} \mathrm{A} 10$-point scale. "significant at $\mathrm{P}<0.05$. GAIS, gout attack intensity score; NRS, numeric rating scale; PGA, patient global assessment; SD, standard deviation; F, F-statistic from one-way analysis of variance; RE, relative efficiency (ratio of $F$-statistics compared with the GAIS).

\section{Responsiveness, and floor and ceiling effects}

As expected, after 5 days improvements were seen in mean scores on all the variables, with all ES being > 0.8. Notably, the sensitivity to change of the GAIS (2.32) was greater compared with other single-item instruments, and only the NRS pain had a slightly higher ES of 2.40 (Table 4). CRP proved to be the weakest measure of change, although the improvement still constituted a large effect (>0.8).

At baseline, no floor effects, defined as $>15 \%$ of patients with the worst possible score, were observed for the GAIS $(1.3 \%)$, or for its component scores pain $(6.6 \%)$, tenderness $(13.2 \%)$ and swelling (10.5\%). Floor effects were also largely absent for the NRS pain and NRS PGA, with $2.6 \%$ and $0 \%$, respectively. As expected, ceiling effects were absent (0\%) for the GAIS, rating scale pain, rating scale swelling, NRS pain and the NRS PGA, and was only $3.9 \%$ for the rating scale tenderness. 
Table 4: Responsiveness to change of self-reported measures between day 1 and day 5

\begin{tabular}{|c|c|c|c|}
\hline & $\mathbf{N}$ & Mean change $\pm S D^{a}$ & ES \\
\hline GAIS & 74 & $1.60 \pm 0.70$ & 2.32 \\
\hline Joint pain ${ }^{b}$ & 74 & $1.66 \pm 0.75$ & 2.21 \\
\hline Joint swelling ${ }^{\mathrm{b}}$ & 74 & $1.49 \pm 0.84$ & 1.76 \\
\hline Joint tenderness ${ }^{b}$ & 74 & $1.66 \pm 0.85$ & 1.95 \\
\hline NRS joint pain ${ }^{c}$ & 74 & $4.37 \pm 1.82$ & 2.40 \\
\hline NRS PGA ${ }^{c}$ & 74 & $2.50 \pm 2.12$ & 1.18 \\
\hline $\mathrm{CRP}^{\mathrm{d}}$ & 71 & $1.12 \pm 1.20$ & 0.94 \\
\hline
\end{tabular}

a Pooled standard deviation (SD). ${ }^{\mathrm{b}}$ Measured on a 5 -point rating scale. ${ }^{\mathrm{c}} \mathrm{A} 10$-point scale. ${ }^{\mathrm{d}}$ Calculated using logtransformed CRP values. GAIS, gout attack intensity score; ES, effect size; PGA, patient global assessment; NRS, numeric rating scale; CRP, C-reactive protein.

\section{DISCUSSION}

In this study we propose a simple, patient-reported gout attack symptom intensity score (GAIS) for use in gout clinical trials. We aimed to develop a new score that includes information that is already frequently collected in clinical trials, with the hope that future studies will report more comprehensive data on gout attack symptom intensity, besides pain alone. Also, by providing a standardized approach to assessing and scoring these gout-related symptoms, it may be easier to compare outcomes across different studies.

Results of our study suggest that combining the single-item components joint pain, swelling and tenderness into an average score, GAIS, yields reliable scores and leads to a more responsive instrument to assess patient-reported gout attack intensity compared with the individual rating scales it is composed of. In fact, responsiveness of the GAIS was rather similar to the NRS pain, which is usually the most responsive instrument in gout clinical trials ${ }^{[8]}$. Furthermore, the results of our Mokken scaling analysis support the proposed GAIS scoring rule of simply summing the individual rating scales. Specifically, these findings imply that the GAIS may be interpreted as an ordinal scale. That is, patients can be rank-ordered with respect to the degree of gout attack intensity they experience using the GAIS total score.

As expected, GAIS was able to better discriminate responders from non-responders, compared with its single-item components. This property is of special importance in clinical trials where the objective is usually to differentiate treatment groups by their level of achieved response. Therefore, our results suggest that researchers intending to use the 5 -point pain rating scale as a primary endpoint in their studies, which is 
not uncommonly done, may consider the GAIS instead ${ }^{[1,3,22,23]}$. This would allow for a more comprehensive assessment of gout attack-related symptoms, and would allow treatment effects to be demonstrated using a smaller numbers of patients.

The current study is also among the first to report on the psychometric properties of all three patient-reported individual symptom rating scales of pain, tenderness and swelling. Thus far, only one previous study examined the measurement properties of the patientreported 4 -point Likert scale pain using data from four randomized controlled trials and one observational study ${ }^{[8]}$. From this study it was concluded that the construct validity and discriminative ability, both the within-group and between-group discrimination, of the 4-point Likert scale pain measure was supported by sufficient evidence. However, in the same study, only instruments assessing physician-reported joint swelling and joint tenderness on a Likert scale were evaluated, while no information for these outcomes assessed by patients were available. In fact, as far as we know, no previous study has done an evaluation of the psychometric properties of self-reported joint swelling and tenderness.

The GAIS is not the first patient-reported outcome measure developed specifically for the gout population. Besides the Tophus Impact Questionnaire ${ }^{[24]}$, Colwell et al. developed the Gout Assessment Questionnaire (GAQ), followed by a second version of this instrument, the GAQ 2.0, by Hirsch et al. ${ }^{[25-27] . ~ T h e ~ G A Q ~} 2.0$ aims to retrospectively assess the impact of gout on health-related quality of life. It also includes two NRS that aim to assess pain and disease activity over the last 4 weeks. As such, it is particularly well suited for cross-sectional studies or to assess change over a relatively longer period of time. The GAIS on the other hand is intended to be administered during a gout attack to collect daily prospective information on gout flare symptom intensity, as part of a gout flare diary. Its intended purpose is to help assess effectiveness of anti-inflammatory treatments in clinical trials or observational studies in patients with acute gout attacks.

A strength of this study is that strict selection criteria were maintained for the trial. Also, only patients with crystal-proven gout, the diagnostic gold-standard for gout, were included. However, this study also had some limitations, including the relatively small sample size. Moreover, most patients were recruited from the rheumatology department at hospital centres in the Netherlands. Therefore, whether these results will hold for populations in, for example, primary care where generally less severe gout patients are seen, remains to be determined in future studies. Another limitation of this study was that we were not yet able to relate GAIS scores to other well-known instruments such as the Health Assessment Questionnaire. Future studies will be needed to demonstrate 
that GAIS scores relate to these measures in expected ways to further support its construct validity.

In conclusion, the present study introduces the GAIS, an average score of three patientreported outcome measures, that may be used in clinical studies for assessing gout attack symptom intensity. The GAIS seems to be a reliable and responsive instrument that represents the intensity of gout attacks by considering patient-reported joint pain, tenderness and swelling.

Acknowledgements

None. 


\section{REFERENCES}

1. Terkeltaub RA, Schumacher HR, Carter JD, Baraf HSB, Evans RR, Wang J, et al. Rilonacept in the treatment of acute gouty arthritis: a randomized, controlled clinical trial using indomethacin as the active comparator. Arthritis Res Ther. 2013;15:R25.

2. Dalbeth N, Jones G, Terkeltaub R, Khanna D, Kopicko J, Bhakta N, et al. Lesinurad, A Selective Uric Acid Reabsorption Inhibitor, in Combination with Febuxostat in Patients with Tophaceous Gout: Findings of a Phase III Clinical Trial. Arthritis Rheumatol. 2017;69(9):1903-13.

3. Schumacher Jr HR, Boice JA, Daikh DI, Mukhopadhyay S, Malmstrom K, Ng J, et al. Randomised double blind trial of etoricoxib and indometacin in treatment of acute gouty arthritis. BMJ. 2002;324(7352):1488-92.

4. Schlesinger N, De Meulemeester M, Pikhlak A, Yücel AE, Richard D, Murphy V, et al. Canakinumab relieves symptoms of acute flares and improves health-related quality of life in patients with difficult-to-treat Gouty Arthritis by suppressing inflammation: results of a randomized, dose-ranging study. Arthritis Res Ther. 2011;13(2):R53.

5. Rubin BR, Burton R, Navarra S, Antigua J, Londono J, Pryhuber KG, et al. Efficacy and Safety Profile of Treatment with Etoricoxib 120 mg Once Daily Compared with Indomethacin 50 mg Three Times Daily in Acute Gout: A Randomized Controlled Trial. Arthritis Rheum. 2004;50(2):598-606.

6. Singh JA, Taylor WJ, Dalbeth N, Simon LS, Sundy J, Grainger R, et al. OMERACT endorsement of measures of outcome for studies of acute gout. J Rheumatol. 2014;41(3):569-73.

7. Schumacher HR, Evans RR, Saag KG, Clower J, Jennings W, Weinstein SP, et al. Rilonacept (interleukin-1 trap) for prevention of gout flares during initiation of uric acid-lowering therapy: Results from a phase III randomized, double-blind, placebo-controlled, confirmatory efficacy study. Arthritis Care Res. 2012;64(10):1462-70.

8. Taylor WJ, Redden D, Dalbeth N, Schumacher HR, Edwards NL, Simon LS, et al. Application of the OMERACT filter to measures of core outcome domains in recent clinical studies of acute gout. J Rheumatol. 2014;41(3):574-80.

9. Jensen MP, Hu X, Potts SL, Gould EM. Single vs composite measures of pain intensity: Relative sensitivity for detecting treatment effects. Pain. 2013;154(4):534-8.

10. Janssen CA, Oude Voshaar MAH, Vonkeman HE, Jansen TLTA, Janssen M, Kok MR, et al. Anakinra for the treatment of acute gout flares: a randomized, double-blind, placebo-controlled, active-comparator, noninferiority trial. Rheumatology. 2019; [Epub ahead of print]. doi.org/10.1093/rheumatology/key402.

11. Sijtsma K, van der Ark LA. A tutorial on how to do a Mokken scale analysis on your test and questionnaire data. Br J Math Stat Psychol. 2017;70(1):137-58.

12. van Schuur WH. Quantitative Applications in the Social Sciences: Ordinal Item Response Theory: Mokken Scale Analysis. Thousand Oaks, CA: SAGE Publications, Inc.; 2011. 128 p.

13. Wind SA. An Instructional Module on Mokken Scale Analysis. Educ Meas Issues Pract. 2017;36(2):50-66.

14. Mokken R, Lewis C. A nonparametric approach to the analysis of dichotomous item responses. Appl Psychol Meas. 1982;6(4):417-30. 
15. Sijtsma K, Molenaar IW. Introduction to Nonparametric Item Response Theory. Thousand Oaks, CA: Sage Publications Inc.; 2002. 176 p.

16. Sijtsma K. On the use, the misuse, and the very limited usefulness of cronbach's alpha. Psychometrika. 2009;74(1):107-20.

17. Ten Berge J, Sočan G. The greatest lower bound to the reliability of a test and the hypothesis of unidimensionality. Psychometrika. 2004;69(4):613-25.

18. Nunnally JC. Psychometric theory. 2nd ed. New York: McGraw-Hill; 1978.

19. Fayers PM, Machin D. Quality of Life: The Assessment, Analysis and Interpretation of Patient-reported Outcomes. 2nd ed. Chichester, England: Wiley; 2007.

20. Cohen J. Statistical Power Analysis for the Behavioural Sciences. 2nd ed. Hillsdale, NJ: Erlbaum; 1988.

21. Terwee CB, Bot SDM, de Boer MR, van der Windt DAWM, Knol DL, Dekker J, et al. Quality criteria were proposed for measurement properties of health status questionnaires. J Clin Epidemiol. 2007;60(1):34-42.

22. Li T, Chen S, Dai Q, Han X, Li Z, Wu D, et al. Etoricoxib versus indometacin in the treatment of Chinese patients with acute gouty arthritis: a randomized double-blind trial. Chin Med J (Engl). 2013;126(10):1867-71.

23. Xu L, Liu S, Guan M, Xue Y. Comparison of Prednisolone, Etoricoxib, and Indomethacin in Treatment of Acute Gouty Arthritis: An Open-Label, Randomized, Controlled Trial. Med Sci Monit. 2016:22:810-7.

24. Aati O, Taylor WJ, Siegert RJ, Horne A, House ME, Tan P, et al. Development of a patient-reported outcome measure of tophus burden: the Tophus Impact Questionnaire (TIQ-20). Ann Rheum Dis. 2015;74(12):2144-50.

25. Colwell HH, Hunt BJ, Pasta DJ, Palo WA, Mathias SD, Joseph-Ridge N. Gout Assessment Questionnaire: Initial results of reliability, validity and responsiveness. Int J Clin Pract. 2006;60(10):1210-7.

26. Hirsch JD, Lee SJ, Terkeltaub R, Khanna D, Singh JA, Sarkin A, et al. Evaluation of an instrument assessing influence of Gout on health-related quality of life. J Rheumatol. 2008;35(12):2406-14.

27. Hirsch JD, Terkeltaub R, Khanna D, Singh J, Sarkin A, Shieh M, et al. Gout disease-specific quality of life and the association with gout characteristics. Patient Relat Outcome Meas. 2010;1:1. 


\section{SUPPLEMENTAL MATERIAL}

Supplemental material 1: Gout Flare Diary

The gout flare diary consist of two parts.

The first part (items 1-3) form the Gout Attack Intensity Score (GAIS). The remaining questions of the flare diary (items 4-6) provide additional patient-reported information on treatment response (item 4), pain (item 5), and a patient's global assessment of overall well-being (item 6).

\section{Part I: GAIS}

The GAIS score is obtained by taking the mean of the summed scored of items 1-3 below. Scores range from 1-5 for each item below as listed by the response options.

1. How much pain is your gout causing you today? (colour in one box completely)

$\square 1$ No pain

$\square 2$ Mild pain

$\square 3$ Moderate pain

$\square 4$ A lot of pain

$\square 5$ Extreme pain

2. How tender to the touch are the gout-affected parts of your body today? (colour in one box completely)

$\square 1$ Not tender

$\square 2$ Slightly tender

$\square 3$ Fairly tender

$\square 4$ Very tender

$\square 5$ Extremely tender

3. How swollen are the gout-affected parts of your body today? (colour in one box completely)

$\square 1$ Not swollen

$\square 2$ Somewhat swollen

$\square 3$ Fairly swollen

$\square 4$ Very swollen

$\square 5$ Extremely swollen

Part II: Other, single-item, instruments included in the gout flare diary

Items 4-6 are scored according to the scores as listed in the response options of each single-item.

4. How do you think your symptoms have changed since the beginning of this gout flare? (colour in one box completely)

$\square 1$ Completely disappeared

$\square 2$ Very much improved

$\square 3$ Much improved

$\square 4$ Somewhat improved

$\square 5$ Unchanged

$\square 6$ Slightly worse

$\square 7$ Much worse

$\square 8$ Very much worse 


\section{Supplemental material 1 continued.}

5. How much pain is your gout causing you today? (colour in one box completely) Absolutely no pain 012345678910 Unbearable pain

6. Taking into account all of the ways your gout is affecting you today, how are you feeling? (colour in one box completely)

Very bad 01123445678910 Very good

Supplemental material 2: Deviations from monotonicity statistically tested at different group sizes across 7 days.

\begin{tabular}{|c|c|c|c|c|c|c|c|}
\hline & \multirow[b]{2}{*}{$\mathrm{N}$} & \multicolumn{3}{|c|}{ Monotonicity ${ }^{a}$ at group size 5} & \multicolumn{3}{|c|}{ Monotonicity a ${ }^{\text {at group size } 20}$} \\
\hline & & Pain & Tenderness & Swelling & Pain & Tenderness & Swelling \\
\hline Day 1 & 76 & $0(32)$ & $0(40)$ & $0(34)$ & $0(0)$ & $0(13)$ & $0(0)$ \\
\hline Day 2 & 82 & $0(0)$ & $0(0)$ & $0(0)$ & $0(0)$ & $0(0)$ & $0(0)$ \\
\hline Day 3 & 85 & $0(0)$ & $0(0)$ & $0(2)$ & $0(0)$ & $0(0)$ & $0(0)$ \\
\hline Day 4 & 83 & $0(0)$ & $0(0)$ & $0(0)$ & $0(0)$ & $0(0)$ & $0(0)$ \\
\hline Day 5 & 86 & $0(-2)$ & $0(0)$ & $0(0)$ & $0(0)$ & $0(0)$ & $0(0)$ \\
\hline Day 6 & 79 & $0(0)$ & $0(0)$ & $0(0)$ & $0(0)$ & $0(0)$ & $0(0)$ \\
\hline Day 7 & 82 & $0(0)$ & $0(0)$ & $0(35)$ & $0(0)$ & $0(0)$ & $0(0)$ \\
\hline
\end{tabular}

a Given as the number of statistical significant deviations from monotonicity (crit-statistic) 


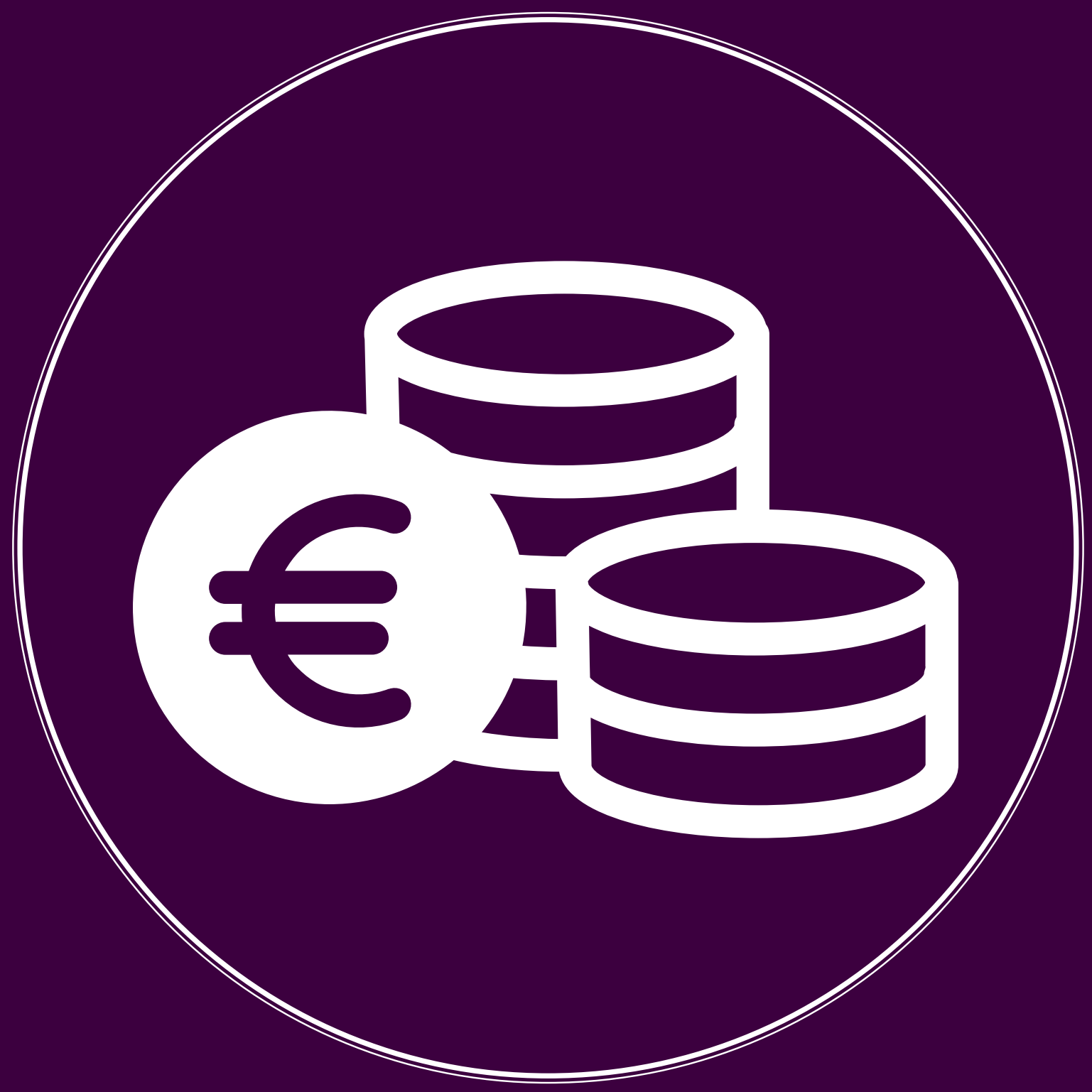




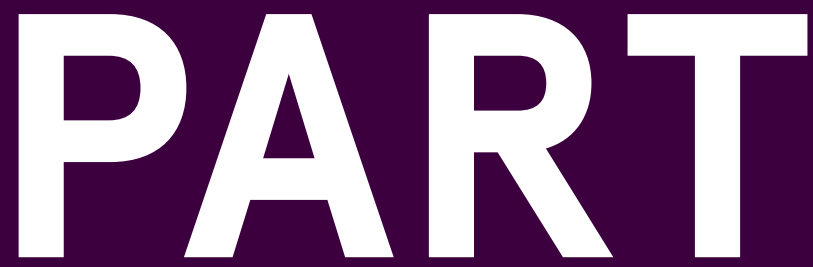

Societal burden of gout 


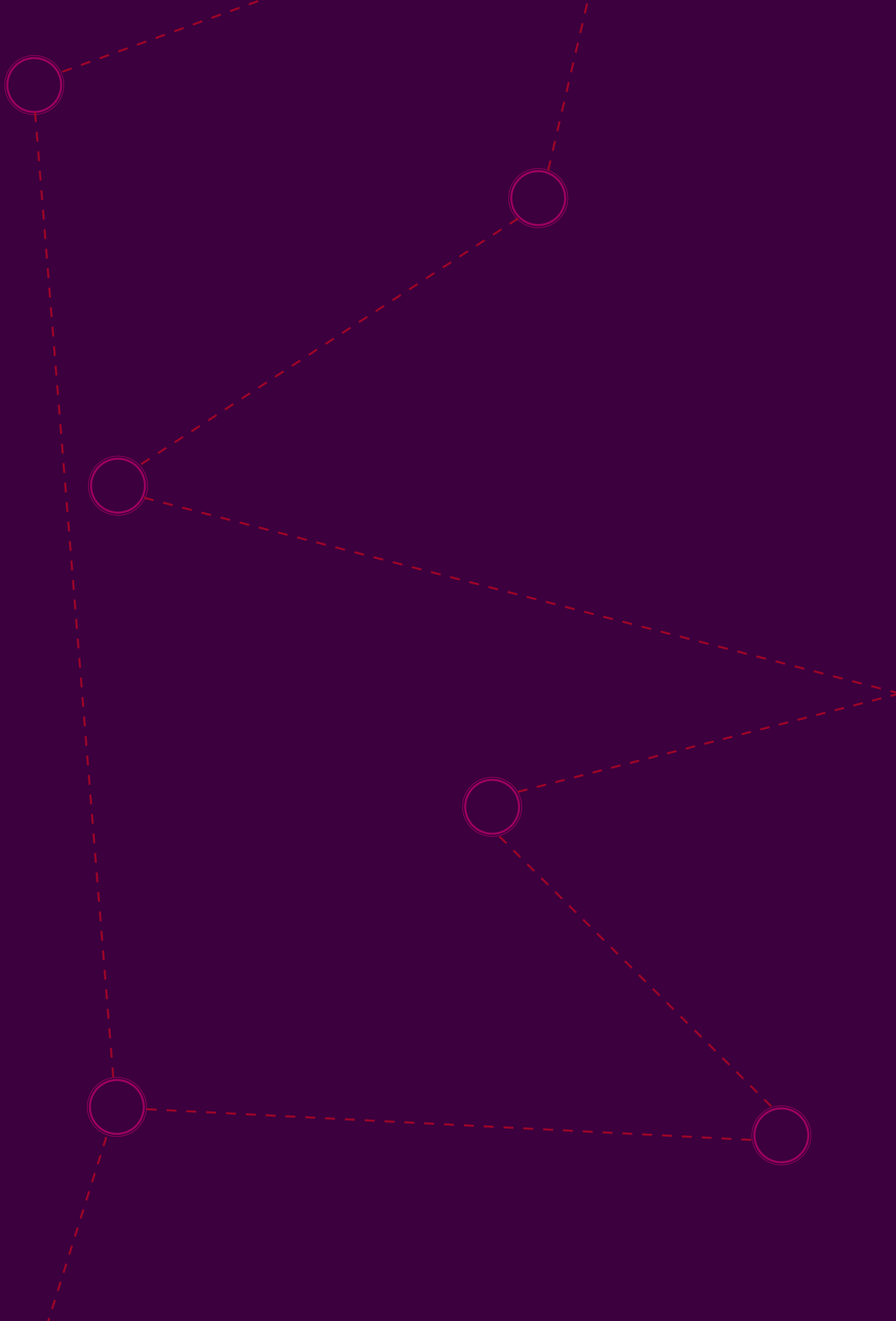




\section{Model-based}

cost-effectiveness analyses

comparing combinations of

urate lowering therapy and anti-inflammatory treatment in newly diagnosed gout patients

Celine J. van de Laar, Carly A. Janssen, Matthijs Janssen, Martijn A.H. Oude Voshaar, Maiwenn J. Al, Mart A.F.J. van de Laar 


\section{ABSTRACT}

Objectives. To assess the cost-effectiveness of various combinations of urate lowering therapy (ULT) and anti-inflammatory treatment in the management of newly diagnosed gout patients, from the Dutch societal perspective.

Methods. A probabilistic patient-level simulation estimating costs and qualityadjusted life years (QALYS) comparing gout and hyperuricemia treatment strategies was performed. In this Markov Model pain was the main determinant of both QALYs and costs, with disutilities assigned to patients who failed to reach the serum urate (SUA) target $(<0.36 \mathrm{mmol} / \mathrm{L})$. Patients faced a daily risk of a gout flare, with a higher risk for patients who failed to achieve the SUA target value. Health states were no flare, and severe pain, mild pain, moderate pain, or no pain in the presence of a flare. Model input was derived from patient level clinical trial data (i.e. SF-6D utility, indirect costs in $€$, pain transition probabilities), meta-analyses or from previously published healtheconomic evaluations. The results of probabilistic sensitivity analyses were presented using incremental cost-effectiveness ratios (ICERs), and summarized using costeffectiveness acceptability curves (CEAC). Scenario analyses were performed for gout patients not necessarily experiencing a gout flare at model entry, and for patients with a higher daily flare probability.

Results. In the base case, the ICER for allopurinol versus no ULT was $€ 1,381$, when combined with naproxen. Febuxostat yielded the highest utility, but also the highest costs (€4,385 vs. €4,063 for allopurinol), resulting in an ICER of $€ 25,173$ when compared to allopurinol. No ULT was not cost-effective, yielding the lowest utility. For the gout flare medications, comparable effects on utility were achieved. Combined with febuxostat, naproxen was the cheapest option $(€ 4,404)$, and anakinra the most expensive $(€ 4,651)$. The ICER of anakinra compared to naproxen was $€ 818,504$. Colchicine and prednisone were dominated by naproxen. Results of the scenario analyses did not change the conclusions drawn from comparison of ULT and anti-inflammatory treatment.

Conclusion. Allopurinol and febuxostat were both cost-effective compared to No ULT. Febuxostat was cost-effective in comparison with allopurinol at higher willingnessto-pay thresholds. For treating acute gout flares, colchicine, naproxen and prednisone offered comparable health economic implications, although naproxen was the favoured option. 


\section{INTRODUCTION}

Gout is an inflammatory response to the presence of hyperuricemia induced monosodium urate (MSU) crystals within the synovial fluid of joints and tissues. It is the most common cause of inflammatory arthritis in men, and reports have shown the burden of gout to be rising ${ }^{[1]}$. Gout attacks are characterized by rapid onset of severe pain and may have a considerable impact on patient's ability to work and function in other social roles ${ }^{[2,3]}$. Typically, gout attacks resolve within 5-7 days with effective anti-inflammatory treatment. Recurrent attacks, and the development of chronic, inflammatory gout, may be prevented by effective urate lowering therapy (ULT) aimed at lowering serum urate (SUA) levels below the saturation point for crystal formation ${ }^{[4]}$. In light of the increasing burden of gout, the importance of optimizing treatment and management of gout at various levels, including patient, community and national, is emphasized.

Various safe and effective therapies are available for the treatment of both gout attacks and hyperuricemia. Allopurinol and febuxostat are currently recommended first-line ULT agents ${ }^{[5]}$. Colchicine, non-steroidal anti-inflammatory drugs (NSAIDs) and glucocorticosteriods are all first-line treatment options for treating acute gout flares ${ }^{[6]}$. Besides these traditional synthetic medications, targeted biological medications, in particular interleukin-1 (IL-1) inhibitors, including anakinra and canakinumab, have been investigated in recent clinical trials for treating gout flares ${ }^{[7,8]}$. IL-1 inhibition is currently recommended as a second-line treatment option for managing gout flares ${ }^{[5,6]}$.

Due to its high prevalence and this increasing use of expensive medications, the health economic implications of novel therapeutic strategies are important to consider when deciding on optimal treatment approaches for patients with gout and hyperuricemia ${ }^{[9]}$. Health economic decision models that have thus far been developed to support such decision making are mainly concerned with the comparison of various ULTs ${ }^{[10-12]}$. Although some models do account for acute gout flares by assigning disutilities, the effects and costs of anti-inflammatory treatments are not explicitly considered in addition to or instead of ULT. However, with the introduction of new, more costly and potentially more effective treatments for treating acute gout flares, simultaneously evaluating outcomes of ULT and anti-inflammatory medications becomes more relevant. In the present study we introduce a new modelling framework for gout, in which the costs and effects of treatment strategies with continuous ULT and anti-inflammatory medications for acute gout flare can be assessed. 


\section{METHODS}

For this study, model-based cost-effectiveness analysis was performed from the societal perspective, in which first-line ULT agents for hyperuricemia (i.e. allopurinol, febuxostat, no ULT), as well as first-line (i.e. colchicine, naproxen and prednisone) and second-line (i.e. anakinra) treatment options for acute gout flares were compared. Analyses were performed from a Dutch perspective. The Dutch Willingness To Pay (WTP)-threshold is not strictly defined and can be calculated based on disease burden. The Consolidated Health Economic Evaluation Reporting Standards (CHEERS) statement was followed in reporting the results of this cost-effectiveness analysis.

\section{Markov Model}

A Markov Model (TreeAge ${ }^{\mathrm{TM}}$ ) was developed to simulate and compare outcomes of various ULT and anti-inflammatory treatment combinations for hyperuricemia and gout flares, respectively (Fig. 1). For the present study the time horizon was one year with a cycle length of one day. Due to the nature of the available data for anakinra and the absence of long-term effects of gout in the model a much longer horizon would not be appropriate. The model considered the one-year course of newly diagnosed gout patients, receiving treatment for their acute gout flare, and who initiated ULT while experiencing a gout flare, reflecting a care path commonly applied for these gout patients in clinical practice. Upon entry in the model, patients were assigned a fixed dose of ULT, with either allopurinol (at $200 \mathrm{mg}$ or $300 \mathrm{mg}$ ), or febuxostat $80 \mathrm{mg}$, or no ULT, based upon available data from the literature. The probability that the patient achieved the SUA target, defined as achieving a SUA level $<0.36 \mathrm{mmol} / \mathrm{L}$, depended on the specific ULT. After it had been determined if a simulated patient would have SUA levels on target $(<0.36 \mathrm{mmol} / \mathrm{L})$ or not on target $(\geq 0.36 \mathrm{mmol} / \mathrm{L})$ for the duration of the simulation, patients experienced a daily risk of having a gout flare. Patients with SUA levels not on target had a higher daily flare risk. When a gout flare was triggered, patients transitioned for seven days between four mutually exclusive pain states (i.e. no pain, mild pain, moderate pain, severe pain) according to transition probabilities defined for each gout flare treatment option. Treatment options included in the model for acute gout flares were colchicine, naproxen, prednisone and anakinra. Dosages were in line with the dosages as used in the clinical studies used as data sources ${ }^{[8,13]}$. No switching between ULT drugs, or medication for acute gout flares, were allowed in the model during the time horizon. Costs and quality-adjusted life years (QALYs) over one year were recorded for all strategies. 


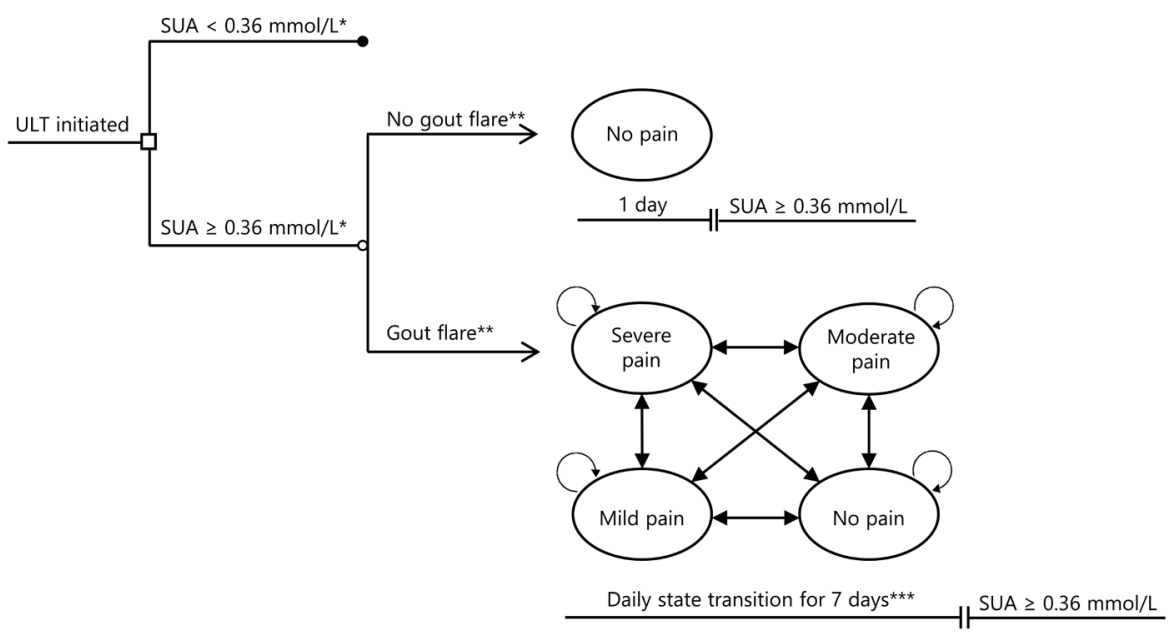

Fig. 1 Model structure

ULT, urate lowering therapy; SUA, serum urate. * Probabilities depend on type of ULT; ** Probabilities depend on SUA status; $* * \star$ Probabilities depend on gout flare medication; $\cdot$ The remainder of the model structure is the same as for the SUA $\geq 0.36 \mathrm{mmol} / \mathrm{L}$ branch

\section{Analyses}

We employed probability sensitivity analyses (PSA) with $2000 \times 200$ model replications to take uncertainty around the point estimates of the model parameters into account. The results were summarized using cost-effectiveness acceptability curves (CEACs). Costs were discounted at $4 \%$, utility was discounted at $1.5 \%$, in concordance with the Dutch costing manual ${ }^{[14]}$. The WTP thresholds in the Netherlands are not explicitly set, but lie between $€ 10,000$ and $€ 80,000$ per QALY ${ }^{[15]}$.

\section{Model Inputs}

All parameters used as input for the model, as well as the data source from which they were estimated, are listed in Table 1 and Table 2. For each input parameter, various distributions were fitted to the observed data. Chi-squared and Anderson Darling fit statistics were used to evaluate goodness of fit. Uncertainty regarding elements of the various transition matrices were expressed using Dirichlet distributions.

\section{Efficacy of ULT}

ULT success was defined as achieving SUA level $<0.36 \mathrm{mmol} / \mathrm{L}$. This SUA target level is recommended by guidelines, supported by reports that have shown that SUA levels below the target level of $0.36 \mathrm{mmol} / \mathrm{L}$ are associated with a decreased risk for gout flares 
${ }^{[6,16]}$. To generate model input, a meta-analysis was performed of ULT clinical trials, in which achieving the SUA target of $<0.36 \mathrm{mmol} / \mathrm{L}$ was one of the endpoints. The indirect adjusted comparison method, using febuxostat $40 \mathrm{mg}$ as the reference treatment was used to obtain efficacy estimates and associated standard errors adjusted for study specific factors ${ }^{[17]}$. For all the placebo arms in the meta-analysis, the percentage of patients achieving the target was zero percent. Therefore for all treatment strategies in which patients do not use ULT it was assumed that no patient achieved the SUA target.

Table 1: Model input

\begin{tabular}{|c|c|c|c|}
\hline Parameter & $\begin{array}{l}\text { Point } \\
\text { estimate }\end{array}$ & Probability distribution & Source \\
\hline \multicolumn{4}{|c|}{ Probability SUA on target ( $<0.36 \mathrm{mmol} / \mathrm{L})$} \\
\hline Allopurinol 200 mg & 0.457 & Beta $(\mu: 0.455, \sigma: 0.045)$ & [19] \\
\hline Allopurinol 300 mg & 0.480 & Beta $(\mu: 0.478, \sigma: 0.027)$ & {$[20-22]$} \\
\hline Febuxostat $80 \mathrm{mg}$ & 0.729 & Beta $(\mu: 0.730, \sigma: 0.013)$ & {$[20,22-25]$} \\
\hline No ULT & 0.000 & $\mathrm{n} / \mathrm{a}$ & [26] \\
\hline \multicolumn{4}{|l|}{ Daily flare probability } \\
\hline SUA on target with ULT & 0.000716 & Beta $(\mu: 0.999, \sigma: 0.002)$ & [12] \\
\hline SUA not on target with ULT & 0.001222 & Beta $(\mu: 0.998, \sigma: 0.002)$ & [12] \\
\hline SUA not on target with no ULT & 0.001637 & Beta $(\mu: 0.998, \sigma: 0.002)$ & [12] \\
\hline \multicolumn{4}{|l|}{ Quality adjusted life days } \\
\hline No pain & 0.86 & Beta (a: $16.325, \beta: 3.076$ ) & [8] \\
\hline Mild pain & 0.77 & Beta (a: 10.942, $\beta: 3.177$ ) & [8] \\
\hline Moderate pain & 0.70 & Beta (a: $12.696, \beta: 5.329$ ) & [8] \\
\hline Severe pain & 0.61 & Beta (a: 19.817, $\beta: 12.877$ ) & [8] \\
\hline \multicolumn{4}{|l|}{ Daily other costs } \\
\hline No pain & 19.95 & $\operatorname{Exp} / g(\lambda: 1.749 \text { /a: 0.317, } \beta: 3.623)^{a}$ & {$[8,14]$} \\
\hline Mild pain & 32.39 & $\operatorname{Exp} / g(\lambda: 1.081 / a: 0.493, \beta: 2.135)^{a}$ & {$[8,14]$} \\
\hline Moderate pain & 58.08 & $\operatorname{Exp} / g(\lambda: 0.599$ /a: 0.434, $\beta:$ 4.149) & {$[8,14]$} \\
\hline Severe pain & 134.32 & $\operatorname{Exp} / g(\lambda: 0.259 \text { /a: 0.291, } \beta: 10.769)^{\mathrm{a}}$ & {$[8,14]$} \\
\hline \multicolumn{4}{|l|}{ Daily drug costs } \\
\hline Colchicine & 0.61 & $\mathrm{n} / \mathrm{a}$ & [27] \\
\hline Naproxen & 0.21 & $\mathrm{n} / \mathrm{a}$ & [27] \\
\hline Prednisone & 0.26 & $\mathrm{n} / \mathrm{a}$ & [27] \\
\hline Anakinra & 33.4 & $\mathrm{n} / \mathrm{a}$ & [27] \\
\hline Allopurinol & 0.13 & $\mathrm{n} / \mathrm{a}$ & [27] \\
\hline Febuxostat & 1.03 & $\mathrm{n} / \mathrm{a}$ & [27] \\
\hline
\end{tabular}

ULT, urate lowering therapy; SUA, serum urate. ${ }^{a} \lambda$-parameter refers to Work Productivity and Activity Impairment questionnaire (exponential distribution) and $a$ and $\beta$ refer to the healthcare resource utilization questionnaire (gamma distribution). 


\section{Flare probabilities}

Daily flare probabilities were calculated from data derived from a previous health economic model by Jutkowitz et al. $2014{ }^{[12]}$. In that paper, annual flare probabilities were given for patients on ULT with controlled SUA $(<0.36 \mathrm{mmol} / \mathrm{L})$, for patients on ULT with uncontrolled SUA ( $\geq 0.36 \mathrm{mmol} / \mathrm{L}$ ), as well as annual flare probabilities for patients not on ULT, with uncontrolled SUA ( $\geq 0.36 \mathrm{mmol} / \mathrm{L}$ ).

\section{Efficacy of flare treatment}

Health states for patients experiencing a gout flare were defined using four pain states (i.e. no pain, mild pain, moderate pain, severe pain), derived from a 4-point pain rating scale that is commonly used as a primary endpoint in gout clinical trials. Inverse variance weighted pain transition probabilities and their standard errors for naproxen and prednisone were obtained by pooling seven day ${ }^{[8]}$, and 90 hour ${ }^{[13]}$ follow up data from two clinical trials. For colchicine and anakinra, the probabilities were obtained from the seven days follow up data of a single trial ${ }^{[8]}$. To avoid empty cells in the transition matrices, due to data sparsity, a Bayesian approach was used in which a transition matrix with 0.5 for each cell (i.e. noninformative prior) was combined with the observed transition frequencies ${ }^{[18]}$. Dirichlet distributions were then fitted on the resulting posterior distribution of transition probabilities in the PSA.

Table 2: Transition matrix for naproxen

\begin{tabular}{lllll}
\hline \multirow{2}{*}{ Start pain state } & \multicolumn{2}{l}{ End pain state (\%) } & & \\
\cline { 2 - 5 } & No & Mild & Moderate & severe \\
\hline No & 91.46 & 6.09 & 1.21 & 1.21 \\
Mild & 13.28 & 80.44 & 4.42 & 1.85 \\
Moderate & 0.87 & 45.61 & 43.86 & 9.65 \\
Severe & 2.17 & 14.13 & 36.96 & 46.74 \\
\hline
\end{tabular}

$\underline{\text { Utilities }}$

Utility weights were estimated for each of the four pain related health states using data obtained from the study by Janssen et al. $2019^{\left[{ }^{8]}\right.}$. The values of utility for each pain state were calculated from the SF-6D. Since a Dutch tariff is unavailable, the SF-6D health stages were valued with the UK tariff.

\section{$\underline{\text { Costs }}$}

We included costs related to gout drug use, healthcare resource utilization and work productivity loss due to gout and other reasons, in euros using Dutch price indices. The 
costs per day for each pain state were determined for all cost variables. The medication costs for the appropriate dosages were derived from the official Dutch list prices ${ }^{[27]}$, and the cost per hour of foregone labour was retrieved from a report by The Netherlands Organizaton for Scientific Research ${ }^{[28]}$.

Healthcare resource utilization for each pain state was estimated using patient-reported questionnaires obtained during a gout clinical trial, wherein patients were asked to report the number of visits to general practitioners, outpatient clinics for specialized caregivers, paramedical caregivers, but also the amount of household care that was used, and diagnostic tests (i.e. echo, CT scan) undergone ${ }^{[8]}$. The costs for each of these items were obtained from the 2015 Dutch Costing manual ${ }^{[14]}$. Outliers, defined as costs deviating more than three standard deviations from the mean, were removed from the healthcare utilization data.

\section{Scenario analyses}

Scenario analyses were performed for gout patients who did not experience a gout flare at model entry, and for gout patients with severe gout. For the latter, the daily flare chances were successively increased.

\section{RESULTS}

As the model used in this study incorporated treatment options for gout flares and hyperuricemia, there were two types of medication (i.e. anti-inflammatory treatment and ULT) compared in this study, resulting in many possible combinations. This section will first focus on different ULT medications combined with naproxen. Hereafter, the cost-effectiveness PSA results of different acute gout flare medication combined with different ULT medication types, will be presented.

\section{PSA Results ULT medication with naproxen as anti-inflammatory treatment}

Table 3 shows the PSA results of three ULT medication types combined with naproxen. No ULT was the cheapest option at $€ 4,031.19$. Allopurinol yielded more utility at a slightly higher cost of $€ 4,063.94$. Compared with no ULT, allopurinol was cost-effective with an incremental cost-effectiveness ratio (ICER) of $€ 1,381.27$. The most expensive option was febuxostat $(€ 4,385.40)$, also yielding the highest utility. Compared to allopurinol, febuxostat had an ICER of $€ 25,173$. At a WTP-threshold of $€ 25,173$, this would be considered cost-effective in The Netherlands. Fig. 2A displays the CEAC. At WTPthresholds below $€ 25,173$, allopurinol is the preferable option. At a WTP-threshold of 
approximately $€ 25,000$, there is a $\sim 50 \%$ chance that allopurinol is cost-effective. At WTP-thresholds above $€ 25,173$, the probability with which febuxostat is cost-effective grows to $\sim 100 \%$, at a WTP of approximately $€ 35,000$. Results and implications were similar when combining the ULT medication with any of the other acute flare medications.

Table 3: Probabilistic Sensitivity Analysis for urate lowering therapy combined with naproxen

\begin{tabular}{llllll}
\hline & Costs $(€)$ & Effects (QALY) & $\Delta \mathrm{C}(\boldsymbol{\epsilon})$ & $\Delta \mathrm{E}(\mathrm{QALY})$ & ICER $(\Delta € / \Delta$ QALY) \\
\hline No ULT + Naproxen & $4,031.19$ & 0.78877 & - & - & - \\
Allopurinol + Naproxen & $4,063.94$ & 0.81248 & 32.75 & 0.02371 & $1,381.27^{*}$ \\
Febuxostat + Naproxen & $4,385.40$ & 0.82525 & 321.46 & 0.01277 & $25,173.06^{\star *}$ \\
\hline
\end{tabular}

QALY, Quality adjusted life years; ICER, incremental cost-effectiveness ratio; ULT, urate lowering therapy; ${ }^{*}$ ICER for allopurinol versus No ULT; ** ICER for febuxostat versus allopurinol.

\section{PSA Results comparing anti-inflammatory treatments with febuxostat as ULT}

The different anti-inflammatory treatments had comparable effects on utility over 1 year when they were combined with febuxostat as ULT. PSA results showed that when naproxen was combined with febuxostat, patients accrued an estimated 0.81 QALYs over the course of 1 year. Larger differences between acute flare medication were seen in costs. Naproxen combined with febuxostat was the cheapest option at an estimated cost of $€ 4,404,-$ per year. Colchicine and prednisone were slightly more expensive at lower accrued utility. Anakinra was the most expensive anti-inflammatory medication. Anakinra did yield higher utility than naproxen, but at an ICER of €818,504 comparing anakinra to naproxen, both combined with febuxostat would not be considered costeffective in the Netherlands with WTP-thresholds ranging from $€ 10,000$ to $€ 80,000$.

The small differences in QALYs gained between acute flare medications (anakinra, colchicine, naproxen, and prednisone) and higher incremental costs of anakinra over the other medications were also reflected in the cost-effectiveness acceptability curves (CEAC) (Fig. 2B). Naproxen had the highest probability of being cost-effective across the full range of WTP-thresholds, ranging around $33 \%$. As the WTP-threshold increases to $€ 80,000$, the probability of naproxen being cost-effective increased to $\sim 42 \%$. However, both colchicine and prednisone had only slightly lower probabilities of being cost-effective than naproxen. Colchicine is stable across the WTP-threshold range at $33 \%$ chance of being cost-effective. Prednisone had a lower probability of being cost-effective and it decreased as the WTP-threshold increases. Table 4 also shows results when acute flare medication is combined with allopurinol and No ULT. In both cases, naproxen is the favourable option. 

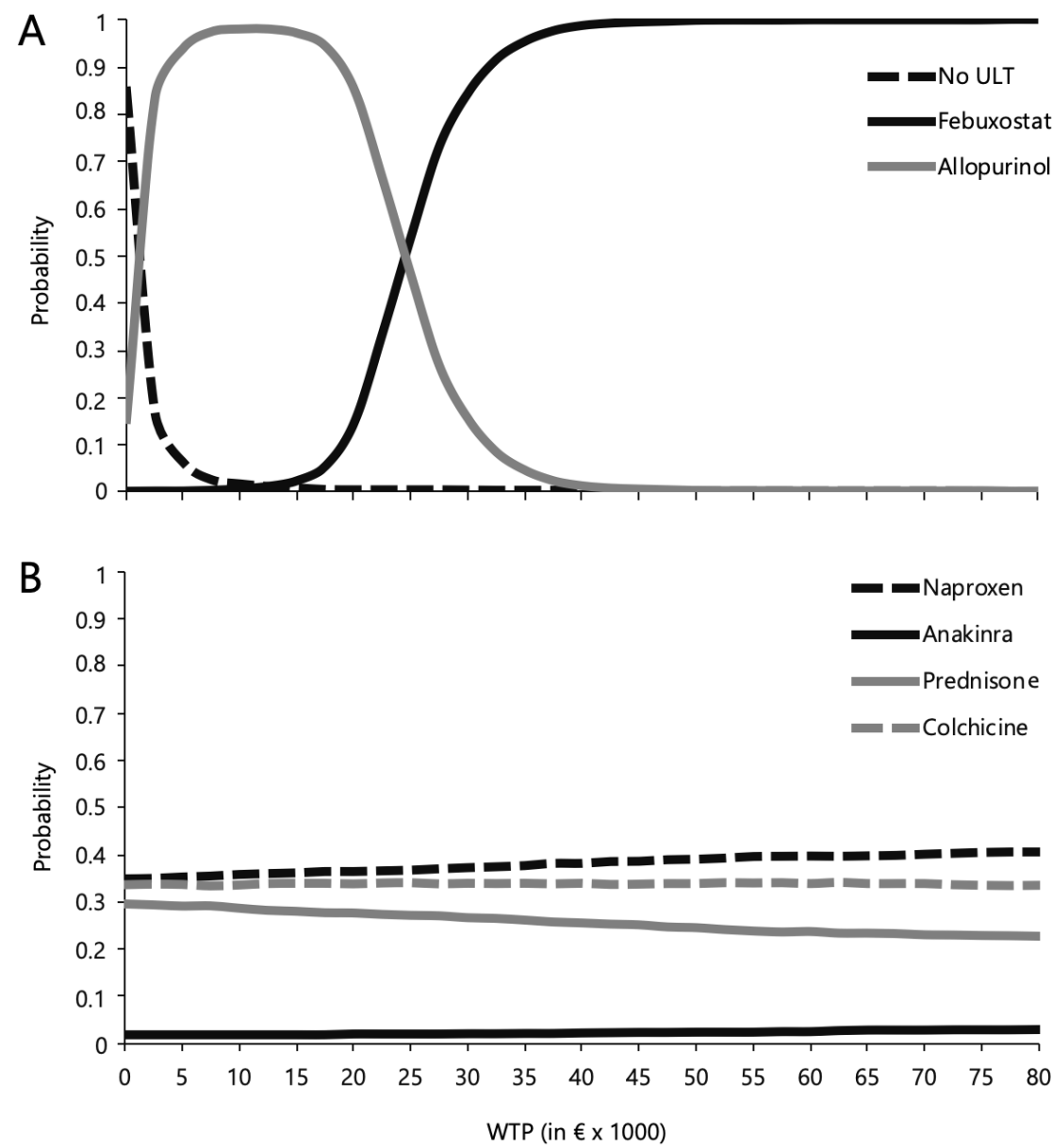

Fig. 2 Cost-effectiveness acceptability curves (CEAC)

ULT, urate lowering therapy; WTP, willingness-to-pay. Panel A displays the CEAC for different ULT combined with naproxen as the anti-inflammatory agent. Panel B displays the CEAC for different anti-inflammatory treatment options combined with febuxostat as the ULT.

\section{Scenario analyses}

In the scenario of patients without flare upon entry, flares per year were lower compared to the base case analysis. This resulted in slightly higher utility and lower costs, but the conclusion remained the same. The second scenario concerns patients with a higher daily probability of starting a flare. Overall this resulted in lower utility and higher costs. Again, the implications did not change. Naproxen remained the most favourable option. The preferred ULT option still depends on the WTP-threshold that would be set in acute gout, but it remained clear that no ULT was not a preferable option (Supplemental material 1). 
Table 4: Probabilistic Sensitivity Analysis for anti-inflammatory treatment

\begin{tabular}{llllll}
\hline & Costs $(€)$ & Effect (QALY) & $\Delta \mathrm{C}(€)$ & $\Delta \mathrm{E}(\mathrm{QALY})$ & ICER \\
\hline Allopurinol & & & & & \\
Naproxen & $4,051.37$ & 0.812493 & - & - & - \\
Colchicine & $4,066.48$ & 0.812356 & 15.11 & -0.00014 & Dominated \\
Prednisone & $4,074.27$ & 0.812356 & 22.9 & -0.00014 & Dominated \\
Anakinra & $4,299.31$ & 0.81274 & 247.94 & 0.00025 & $1,003,805.67$ \\
Febuxostat & & & & & \\
Naproxen & $4,404.72$ & 0.82288 & - & - & - \\
Colchicine & $4,426.91$ & 0.82274 & 22.19 & -0.00014 & Dominated \\
Prednisone & $4,424.72$ & 0.82277 & 20.00 & -0.00011 & Dominated \\
Anakinra & $4,651.09$ & 0.82318 & 246.37 & 0.00030 & $818,504.98$ \\
No ULT & & & & & \\
Naproxen & $4,012.99$ & 0.788082 & - & - & - \\
Colchicine & $4,039.99$ & 0.787918 & 27.00 & -0.00016 & Dominated \\
Prednisone & $4,040.82$ & 0.757973 & 27.83 & -0.03011 & Dominated \\
Anakinra & $4,297.02$ & 0.788356 & 284.03 & 0.00027 & $1,036,605.84$ \\
\hline AalY, Quality & & & & &
\end{tabular}

QALY, Quality adjusted life years; ICER, incremental cost-effectiveness ratio; ULT, urate lowering therapy.

\section{DISCUSSION}

A health economic model was developed for evaluating the costs and effects related to gout treatment strategies that simultaneously covers anti-inflammatory agents for acute gout flares (i.e. colchicine, naproxen, prednisone, anakinra) and ULT options for hyperuricemia (i.e. allopurinol, febuxostat, or no ULT).

The results of our comparison of ULT strategies suggest that strategies in which no ULT is used would not be considered cost-effective at any WTP threshold that is customary in the Netherlands. This finding supports, from a health-economic point of view, the 2016 updated EULAR guidelines, which was the first to emphasize that ULT should be considered and discussed with every patient from the first presentation of gout with a definite diagnosis ${ }^{[6]}$. Our results further show that which specific ULT yields the highest net benefit depends on the WTP threshold. In the Netherlands, the WTP threshold ranges from $€ 10,000$ to $€ 80,000$ and depends on the 'burden of disease', estimated using the proportional shortfall method. The ICER of febuxostat compared to allopurinol is $€ 25,173.06$, and is thus quite close to the WTP-threshold set for the lowest disease burden category, which is up to $€ 20,000$ euro per QALY ${ }^{[15]}$. Although not yet explicitly defined, the disease burden of the population of gout patients considered in this study could be expected to fall in the lowest category defined by the National Healthcare 
Institute ${ }^{[15]}$ due to its episodic pattern with longer periods of no attacks. However, there are various methods of calculating burden of disease ${ }^{[1]}$. With respect to the costeffectiveness of different ULT, this would indicate that using allopurinol is the preferable option. Moreover, the disease burden of gout varies substantially with severity. For example health-related quality of life of patients with difficult to treat, chronic gout was found to be similar to that of patients with active rheumatoid arthritis ${ }^{[29]}$. Since the proportional shortfall weighted burden of rheumatoid arthritis corresponds to the highest disease burden category [30], a WTP threshold of $€ 80,000$ could be expected to also apply to the population of patients with severe gout. This would suggest that febuxostat may be preferable to allopurinol in the treatment of chronic gout. However, as the results of our study were not based on patients with severe gout, this would need to be investigated further in future studies.

The cost-effectiveness of various ULT monotherapies has been compared in three previous, model-based studies ${ }^{[10-12]}$. In all cases, these studies considered ULT only and, either did not consider the impact of flares on quality of life or used a disutility to account for flares. Furthermore, utility and costs weights were attached to various SUA level related health states. By contrast, in our model utility and costs are mainly determined by the current level of pain experienced by the patient, with a disutility for patients not reaching the SUA target. This choice was motivated by the consistent finding in previous studies that pain is strongly related to health-related quality of life of gout patients, whereas mixed findings were reported with respect to the relationship between SUA levels and quality of life ${ }^{[31]}$. The assumption that lowering SUA levels would produce utility gains independently of gout flares was also considered implausible in a recent NICE single technology appraisal of cost-effectiveness evidence in favour of febuxostat, since gout is usually asymptomatic in between acute flares ${ }^{[32]}$. Nevertheless, it is interesting to note that despite these differences in model structure, all three studies found results roughly consistent with ours in that applying any ULT was found to be costeffective relative to no ULT. Furthermore, febuxostat was consistently associated with both higher costs and higher effectiveness compared with allopurinol in the previous studies. Several more studies evaluated cost-effectiveness of various ULT sequences

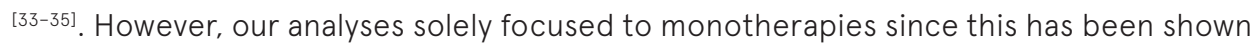
to be the most common treatment pattern in clinical practice ${ }^{[12,36]}$. It should be noted though that various international guidelines currently recommend titrating allopurinol dosages up to $900 \mathrm{mg} /$ day. It seems likely that a higher percentage of patients would be able to reach the SUA target at higher allopurinol dosages. Unfortunately, no suitable data was identified in our literature review to be able to assess this treatment strategy in our model. 
To the best of our knowledge, the current study is also the first model-based study to examine cost-effectiveness of anti-inflammatory treatments for gout attacks. Results of our study reveal that naproxen was the favourable treatment at any WTP-threshold, in combination with any of the ULT, although overall differences in cost-effectiveness between conventional treatment strategies remained small. In addition, our results showed that treatment with anakinra, although accruing slightly higher health outcomes after one year compared to conventional treatments, was not cost-effective, primarily driven by its high costs per treatment. Costs over one year for strategies including anakinra were approximately $€ 200$,- higher than the other acute gout flare medications. Although this is a smaller difference than what the difference in absolute drug prices between anakinra and, for example, naproxen would suggest, our findings do not support a role for anakinra as a first line treatment in the overall gout population.

The current study had some limitations. First, the amount of data used to estimate the pain transition probabilities for each acute gout flare treatment option, was limited. This resulted from the need to have access to patient level data to populate the model. In particular, data with regard to anakinra and colchicine were based on a single randomized controlled trial. The resulting uncertainty about the relative effectiveness of the different treatments may have undermined our ability to differentiate the efficacy of different anti-inflammatory treatments. Second, the occurrence of (serious) adverse events and their associated costs and consequence on utilities, were not included in the model. This also applies for using prophylaxis when initiation ULT as recommended by gout guidelines. Insufficient data was available for us to consider the IL-1 inhibitor, canakinumab, for the treatment of acute gout flares, or second-line ULT agents as pegloticase and lesinurad, which has just recently been approved by the Food and Drug Administration in combination with allopurinol ${ }^{[37]}$. However, none of these drugs are likely to become first line treatment options for gout and hyperuricemia in the near future.

In conclusion, the findings of our study show that ULT, with either allopurinol or febuxostat, are cost-effective first-line ULT agents for treating hyperuricemia. For the treatment of acute gout flares, conventional first-line treatments (i.e. colchicine, naproxen, prednisone) had similar health economic implications, of which naproxen had the most favourable costs and effects profile.

\section{Acknowledgements}

None. 


\section{REFERENCES}

1. Smith E, Hoy D, Cross M, Merriman TR, Vos T, Buchbinder R, et al. The global burden of gout: estimates from the Global Burden of Disease 2010 study. Ann Rheum Dis. 2014;73(8):1470-6.

2. Becker MA, Schumacher HR, Benjamin KL, Gorevic P, Greenwald M, Fessel J, et al. Quality of life and disability in patients with treatment-failure gout. J Rheumatol. 2009;36(5):1041-8.

3. Edwards NL, Sundy JS, Forsythe A, Blume S, Pan F, Becker MA. Work productivity loss due to flares in patients with chronic gout refractory to conventional therapy. J Med Econ. 2011;14(1):10-5.

4. Bursill D, Taylor WJ, Terkeltaub R, Kuwabara M, Merriman TR, Grainger R, et al. Gout, Hyperuricemia, and Crystal-Associated Disease Network Consensus Statement Regarding Labels and Definitions for Disease Elements in Gout. Arthritis Care Res (Hoboken). 2019;71(3):427-34.

5. Khanna D, Fitzgerald JD, Khanna PP, Bae S, Singh MK, Neogi T, et al. 2012 American college of rheumatology guidelines for management of gout. part 1: Systematic nonpharmacologic and pharmacologic therapeutic approaches to hyperuricemia. Arthritis Care Res. 2012;64(10):1431-46.

6. Richette P, Doherty M, Pascual E, Barskova V, Becce F, Castañeda-Sanabria J, et al. 2016 updated EULAR evidence-based recommendations for the management of gout. Ann Rheum Dis. 2017;76(1):29-42.

7. Pascart T, Richette P. Current and future therapies for gout. Expert Opin Pharmacother. 2017;18(12):1201-11.

8. Janssen CA, Oude Voshaar MAH, Vonkeman HE, Jansen TLTA, Janssen M, Kok MR, et al. Anakinra for the treatment of acute gout flares: a randomized, double-blind, placebo-controlled, active-comparator, noninferiority trial. Rheumatology. 2019; [Epub ahead of print]. doi.org/10.1093/rheumatology/key402.

9. Helmick CG, Felson DT, Lawrence RC, Gabriel S, Hirsch R, Kwoh CK, et al. Estimates of the prevalence of arthritis and other rheumatic conditions in the United States. Part I. Arthritis Rheum. 2008;

10. Gandhi PK, Gentry WM, Ma Q, Bottorff MB. Cost-effectiveness analysis of allopurinol versus febuxostat in chronic gout patients: a U.S. payer perspective. J Manag care Spec Pharm. 2015;21(2):165-75.

11. Smolen LJ, Gahn JC, Mitri G, Shiozawa A. Febuxostat in the management of gout: a cost-effectiveness analysis. J Med Econ. 2016;19(3):265-76.

12. Jutkowitz E, Choi HK, Pizzi LT, Kuntz KM. Cost-effectiveness of allopurinol and febuxostat for the management of gout. Ann Intern Med. 2014;161(9):617-26

13. Janssens HJ, Janssen M, van de Lisdonk EH, van Riel PL, van Weel C. Use of oral prednisolone or naproxen for the treatment of gout arthritis: a double-blind, randomised equivalence trial. Lancet. 2008;371(9627):185460.

14. Hakkaart-van Roijen L, van der Linden N, Bouwmans C, Kanters T, Tan S. Kostenhandleiding. Methodologie van kostenonderzoek en referentieprijzen voor economische evaluaties in de gezondheidszorg. 2015.

15. Saase L, Zwaap J, Knies S, Meijden van der C, Staal P, Heiden van der L. Cost-effectiveness in daily practice. $2015 ; 64$.

16. Sarawate CA, Patel PA, Schumacher HR, Yang W, Brewer KK, Bakst AW. Serum Urate Levels and Gout Flares Analysis from managed care data. J Clin Rheumatol. 2006;12(2):61-5.

17. Song F, Altman DG, Glenny A-M, Deeks JJ. Validity of indirect comparison for estimating efficacy of 
competing interventions: empirical evidence from published meta-analyses. Bmj. 2003;326:472-472.

18. Briggs AH, Ades AE, Price MJ. Probabilistic sensitivity analysis for decision trees with multiple branches: Use of the Dirichlet distribution in a Bayesian framework. Medical Decision Making. 2003.

19. Kamatani N, Fujimori S, Hada T, Hosoya T, Kohri K, Nakamura T, et al. An Allopurinol-Controlled, Randomized, Double-Dummy, Double-Blind, Parallel Between-Group, Comparative Study of Febuxostat (TMX-67), a NonYPurine-Selective Inhibitor of Xanthine Oxidase, in Patients With Hyperuricemia Including Those With Gout in Japan. P. J Clin Rheumatol. 2011;17(4):S13-8.

20. Huang X, Du H, Gu J, Zhao D, Jiang L, Li X, et al. An allopurinol-controlled, multicenter, randomized, double-blind, parallel between-group, comparative study of febuxostat in Chinese patients with gout and hyperuricemia. Int J Rheum Dis. 2014;17(6):679-86

21. Kamatani N, Fujimori S, Hada T, Hosoya T, Kohri K, Nakamura T, et al. An allopurinol-controlled, multicenter, randomized, open-label, parallel between-group, comparative study of febuxostat (TMX-67), a non-purineselective inhibitor of xanthine oxidase, in patients with hyperuricemia including those with gout in Japan. J Clin Rheumatol. 2011;17(4):S44-9.

22. Xu S, Liu X, Ming J, Chen S, Wang Y, Liu H, et al. A phase 3, multicenter, randomized, allopurinol-controlled study assessing the safety and efficacy of oral febuxostat in Chinese gout patients with hyperuricemia. 2015;18:669-78.

23. Kamatani N, Fujimori S, Hada T, Hosoya T, Kohri K, Nakamura T, et al. Placebo-Controlled Double-Blind Dose-Response Study of the Non-Purine-Selective Xanthine Oxidase Inhibitor Febuxostat (TMX-67) in Patients With Hyperuricemia (Including Gout Patients) in Japan. J Clin Rheumatol. 2011;17(4):S35-43.

24. Becker MA, Schumacher HR, Espinoza LR, Wells AF, MacDonald P, Lloyd E, et al. The urate-lowering efficacy and safety of febuxostat in the treatment of the hyperuricemia of gout: the CONFIRMS trial. Arthritis Res Ther. 2010;12(2):R63.

25. Becker MA, Schumacher HR, Wortmann RL, MacDonald PA, Palo WA, Eustace D, et al. Febuxostat, a novel nonpurine selective inhibitor of xanthine oxidase: A twenty-eight-day, multicenter, phase II, randomized, double-blind, placebo-controlled, dose-response clinical trial examining safety and efficacy in patients with gout. Arthritis Rheum. 2005;52(3):916-23.

26. Li S, Yang H, Guo Y, Wei F, Yang X, Li D, et al. Comparative efficacy and safety of urate-lowering therapy for the treatment of hyperuricemia: A systematic review and network meta-analysis. Scientific Reports. 2016.

27. Zorginstituut Nederland. Drug prescription costs. [Internet. Accessed SEPTEMBER 27th, 2018. Website in Dutch] Available from: www.medicijnkosten.nl.

28. van der Ploeg, C.P.B., Pal, S., Vroome, E.D., Bossche S. De kosten van ziekteverzuim voor werkgevers in nederland [Internet]. 2014. Available from: http://www.stresspreventionatwork.nl/downloads/brochure_ kosten_ZV_NEA.pdf

29. Bardin T, Voshaar MAHO, van de Laar MAFJ. The Human and Economic Burden of Difficult-to-Treat Gouty Arthritis. Jt Bone Spine. 2015;

30. Lindemark F, Norheim OF, Johansson KA. Making use of equity sensitive QALYs: A case study on identifying the worse off across diseases. Cost Eff Resour Alloc. 2014; 
31. Chandratre P, Roddy E, Clarson L, Richardson J, Hider SL, Mallen CD. Health-related quality of life in gout: a systematic review. Rheumatology. 2013;52(11):2031-40

32. Stevenson M, Pandor A. Febuxostat for the management of hyperuricaemia in patients with gout: A nice single technology appraisal. Pharmacoeconomics. 2011;

33. Perez-Ruiz F, Díaz-Torné C, Carcedo D. Cost-effectiveness analysis of febuxostat in patients with gout in Spain. J Med Econ. 2016;19(6):604-10

34. Beard SM, von Scheele BG, Nuki G, Pearson I V. Cost-effectiveness of febuxostat in chronic gout. Eur J Heal Econ. 2014;15(5):453-63.

35. Ruggeri M, Basile M, Drago C, Rolli FR, Cicchetti A. Cost-Effectiveness Analysis of Lesinurad/Allopurinol Versus Febuxostat for the Management of Gout/Hyperuricemia in Italy. Pharmacoeconomics. 2018;36(5):625-36.

36. Sarawate CA, Brewer KK, Yang W, Patel PA, Schumacher HR, Saag KG, et al. Gout medication treatment patterns and adherence to standards of care from a managed care perspective. Mayo Clin Proc. 2006:

37. Lesinurad/Allopurinol (Duzallo) for Gout-Associated HyperuricemiaLesinurad/Allopurinol (Duzallo) for Gout-Associated HyperuricemiaLesinurad/Allopurinol (Duzallo) for Gout-Associated Hyperuricemia. JAMA. 2018 Jan;319(2):188-9. 


\section{SUPPLEMENTAL MATERIAL}

Supplemental material 1: Results of scenario analyses

Table S1: No flare upon entry full results

\begin{tabular}{|c|c|c|c|c|c|}
\hline & Costs $(€)$ & Effects (QALY) & $\Delta \mathrm{C}(€)$ & $\Delta \mathrm{E}(\mathrm{QALY})$ & ICER $(\Delta \mathrm{C} / \Delta \mathrm{E})$ \\
\hline \multicolumn{6}{|l|}{ Febuxostat } \\
\hline Naproxen & 4,146 & 0.82484 & - & - & - \\
\hline Colchicine & 4,152 & 0.82481 & 5.95 & -0.00003 & Dominated \\
\hline Prednisone & 4,147 & 0.82481 & 0.42 & -0.00003 & Dominated \\
\hline Anakinra & 4,217 & 0.82482 & 71.19 & -0.00002 & Dominated \\
\hline \multicolumn{6}{|l|}{ Allopurinol } \\
\hline Naproxen & 3,561 & 0.82436 & - & - & - \\
\hline Colchicine & 3,566 & 0.82426 & 4.77 & -0.00010 & Dominated \\
\hline Prednisone & 3,563 & 0.82431 & 1.54 & -0.00005 & Dominated \\
\hline Anakinra & 3,643 & 0.82431 & 81.70 & -0.00005 & Dominated \\
\hline \multicolumn{6}{|l|}{ No ULT } \\
\hline Naproxen & 3,885 & 0.78639 & - & - & - \\
\hline Colchicine & 3,894 & 0.78632 & 8.49 & -0.00007 & Dominated \\
\hline Prednisone & 3,893 & 0.78634 & 7.67 & -0.00005 & Dominated \\
\hline Anakinra & 3,989 & 0.78636 & 103.91 & -0.00003 & Dominated \\
\hline \multicolumn{6}{|l|}{ ULT comparison } \\
\hline No ULT + Naproxen & 3,885 & 0.78639 & - & - & - \\
\hline Allopurinol + Naproxen & 3,561 & 0.82436 & -324 & 0.03797 & Dominating* \\
\hline Febuxostat + Naproxen & 4,146 & 0.82484 & 585 & 0.00048 & $1,218,750$ ** \\
\hline
\end{tabular}

QALY, Quality adjusted life years; ICER, incremental cost-effectiveness ratio; ULT, urate lowering therapy; * ICER for allopurinol versus No ULT; ** ICER for febuxostat versus allopurinol. 
Table S2: Increase flare chance full results

\begin{tabular}{|c|c|c|c|c|c|}
\hline & Costs $(€)$ & Effects (QALY) & $\Delta \mathrm{C}(€)$ & $\triangle E(Q A L Y)$ & ICER $(\Delta \mathrm{C} / \Delta \mathrm{E})$ \\
\hline \multicolumn{6}{|l|}{ Febuxostat } \\
\hline Naproxen & 4,389 & 0.82507 & - & - & - \\
\hline Colchicine & 4,417 & 0.82492 & 28.25 & -0.00015 & Dominated \\
\hline Prednisone & 4,406 & 0.82493 & 17.05 & -0.00014 & Dominated \\
\hline Anakinra & 4,642 & 0.82535 & 252.85 & 0.00027 & 927,118 \\
\hline \multicolumn{6}{|l|}{ Allopurinol } \\
\hline Naproxen & 4,069 & 0.80843 & - & - & - \\
\hline Colchicine & 4,099 & 0.80829 & 29.22 & -0.00013 & Dominated \\
\hline Prednisone & 4,090 & 0.80828 & 20.23 & -0.00014 & Dominated \\
\hline Anakinra & 4,336 & 0.80872 & 266.45 & 0.00030 & 896,226 \\
\hline \multicolumn{6}{|l|}{ No ULT } \\
\hline Naproxen & 4,043 & 0.78905 & - & - & - \\
\hline Colchicine & 4,075 & 0.78889 & 32.34 & -0.00015 & Dominated \\
\hline Prednisone & 4,076 & 0.78890 & 33.02 & -0.00015 & Dominated \\
\hline Anakinra & 4,331 & 0.78934 & 288.38 & 0.00029 & 978,458 \\
\hline \multicolumn{6}{|l|}{ ULT Comparison } \\
\hline No ULT + naproxen & 4,043 & 0.78905 & - & - & - \\
\hline Allopurinol + naproxen & 4,069 & 0.80843 & 26 & 0.01938 & $1,342^{*}$ \\
\hline Febuxostat + naproxen & 4,389 & 0.82507 & 320 & 0.01664 & $19,231^{\star *}$ \\
\hline
\end{tabular}

QALY, Quality adjusted life years; ICER, incremental cost-effectiveness ratio; ULT, urate lowering therapy; * ICER for allopurinol versus No ULT; ** ICER for febuxostat versus allopurinol. 
<smiles>CO[C@H]1O[C@H](O)[C@H](O)O1</smiles> 
Summary \& General Discussion 


\section{SUMMARY AND MAIN FINDINGS}

\section{Part I: Clinical management of gout and hyperuricemia}

The study in Chapter 2 aimed to answer the question whether a 5-day, $100 \mathrm{mg}$ daily, subcutaneous injection of the interleukin-1 (IL-1) receptor antagonist anakinra is an effective and safe treatment alternative for treating acute gout flares. For this purpose, a multicenter, double-blind, double-dummy, randomized, active-controlled, noninferiority trial was designed and conducted in The Netherlands, in which anakinra was compared to treatment as usual (colchicine, naproxen or prednisone). In total, 88 patients were included, of which 45 and 43 patients were treated with treatment as usual or anakinra, respectively. Anakinra was found to be non-inferior to treatment as usual in reducing the pain associated with acute gout flares. Other gout-related symptoms such as joint tenderness and joint swelling also decreased to a similar extent over days one to five in both treatment arms. This study suggests that anakinra is an effective IL-1 inhibitor for the treatment of acute gout flares.

Gout flare recurrences are rather common in gout patients that are on urate lowering therapy (ULT), particularly in the time period directly after starting ULT. In the study in Chapter 3, the prognostic value of various clinical, demographic and patient-reported factors for predicting early gout flare recurrences was investigated, in patients initiating ULT during an acute gout flare $(\mathrm{N}=75)$. Within three months post baseline, $48 \%(\mathrm{~N}=36)$ of patients experienced a gout flare recurrence. Results of the binary multivariate regression analysis revealed that lack of prophylaxis when starting ULT (odds ratio (OR) 11.56) and c-reactive protein > $30 \mathrm{mg} / \mathrm{L}$ (OR 9.47) were independently predictive of gout flare recurrences $\leq 3$ months post baseline.

At present, the potential added value of adopting a treat-to-target (T2T) strategy in clinical practice in lowering serum urate (SUA) levels in gout patients, has not yet been studied in much detail. Chapter $\mathbf{4}$ presents the results of a clinical audit regarding the use of a targeted treatment approach in real-world gout patients using currently available ULT, in reaching the European League Against Rheumatism defined SUA level targets. This retrospective, medical chart-review that included gout patients from two hospital centers in The Netherlands, found that the SUA target $<360 \mu \mathrm{mol} / \mathrm{L}$ was reached in $83 \%$ of the cases treated in daily clinical practice with use of currently available ULT and a targeted treatment approach. These results provide a proof-of-concept of a T2T strategy in gout, and suggest that adoption of this strategy in clinical practice might contribute to increasing the proportion of gout patients having SUA levels on target. 
In Chapter 5, medication prescription records from a nationally-representative database were retrospectively analyzed in order to determine the levels of compliance and persistence to dispensed ULT for the treatment of gout and hyperuricemia. After one year, it was shown that $51.7 \%$ of the patients were categorized as compliant by having ULT coverage for $\geq 80 \%$ of the days in one year, while only $42.7 \%$ of the patients were still continuing their ULT medication after one year (persistent), without having surpassed a refill gap of 30 days. Increasing age, being male and initial dispensed prescription of ULT by a rheumatologist were factors associated with increasing compliance and persistence. As ULT is proposed as a life-long treatment by gout management guidelines, findings from this study suggest that ULT medication compliance and persistence is suboptimal in the Netherlands, and suggest that investigating reasons for treatment cessation and ways to enhance medication adherence to ULT are desired.

\section{Part II: Patient-reported outcome measures (PROMs) in gout}

In Chapter 6, a systematic literature review is presented that aimed to identify all the available PROMs used in gout, and to critically review their content validity and measurement properties (i.e. reliability, responsiveness, construct validity, floor and ceiling effects). The search resulted in the inclusion of 13 PROMs, covering almost all outcome domains that have been endorsed for use in acute and chronic gout clinical studies. Noticeable, no PROMs were available to assess patient-reported joint swelling and joint tenderness, and only three PROMs were gout-specific. Results from this study showed that the physical functioning subscale of the Short Form-36 item version 2 was the only PROM which had sufficient evidence to support all psychometric properties that were examined for this study. Overall, this study showed that for many of the PROMs used in gout clinical research, the psychometric properties are not yet well supported.

The objective of the study in Chapter 7 was to develop an average score representing a patient's overall level of gout flare symptom intensity, the Gout Attacg Intensity Score (GAIS), composed of individual symptom scores of patient-reported pain, swelling and tenderness, measured on a 5-point rating scale. Findings showed that the GAIS can be interpreted as an ordinal scale, wherein higher scores indicate higher levels of gout flare intensity. The GAIS was shown to be a reliable and responsive instrument, and it was able to better discriminate between responders and non-responders compared to its single-item counterparts. Results of the study suggest that the GAIS may be used in gout clinical studies for assessing gout flare intensity.

\section{Part III: Societal burden of gout}

In Chapter 8, a health economic analysis based on a patient-level simulation was 
performed, following the one-year course of newly diagnosed gout patients initiating ULT during an acute gout flare for determining the cost-effectiveness of different combinations of anti-inflammatory treatment for acute gout flares and ULT. The model is the first in gout to simultaneously evaluate these treatments, and to include gout flares as a main determinant for costs and utilities. Compared to No ULT, both allopurinol and febuxostat were cost-effective, and at higher willingness-to-pay thresholds (approximately > €25,173) febuxostat was cost-effective in comparison to allopurinol, in combination with different anti-inflammatory agents. For treating acute gout flares, and independent of which ULT used, colchicine, naproxen and prednisone offered similar health economic implications, although naproxen was overall most favored. Anakinra was not cost-effective compared to conventional treatments, driven by its high costs per treatment, but did yield the highest utility.

\section{GENERAL DISCUSSION AND FUTURE DIRECTIONS}

\section{Interleukin-1 (IL-1) inhibitors in the management of gout}

Evidence supporting the role of $\mathrm{IL}-1$ in the pathogenesis of gout flares has emerged from various animal and in-vitro studies over the past decades ${ }^{[1]}$. More recently, several studies have attempted to further validate this concept in clinical trials of IL-1 inhibitors in human subjects with gout. However, in studies performed prior to this thesis, mixed results have been observed with respect to the efficacies of various IL-1 inhibitors in gout. The efficacy of the anti-body canakinumab, which neutralizes IL-1 $\beta$, was examined in two studies ${ }^{[2,3]}$, while another randomized study examined the efficacy of the soluble $\mathrm{IL}-1$ Trap rilonacept, neutralizing both $\mathrm{IL}-1 \beta$ and IL-1 ${ }^{[4]}$. In the latter, a single dose of subcutaneous $320 \mathrm{mg}$ rilonacept was given as an add-on therapy to indomethacin $50 \mathrm{mg}$ three times daily. The study failed to demonstrate superiority of this regimen compared to indomethacin alone in reducing pain 72 hours after starting treatment in patients with acute gout flares ${ }^{[4]}$. Notable about this study is that the authors chose to test for superiority of rilonacept when given as an add-on therapy to an optimally dosed non-steroidal anti-inflammatory drug (NSAID). This was a surprising, ambitious choice, given the already well-documented rapid and strong pain-relieving effects of NSAIDs in patients with acute gout flares ${ }^{[5,6]}$. On the other hand, two randomized controlled trials showed that a single subcutaneous injection of $150 \mathrm{mg}$ canakinumab was superior in reducing pain after 72 hours compared to a single intramuscular injection of triamcinolone acetonide $40 \mathrm{mg}$ in patients who could not be (successfully) treated with NSAID and/or colchicine ${ }^{[3]}$. However, it has been questioned whether the choice and dose of the comparator, triamcinolone acetonide $40 \mathrm{mg}$, were optimal in the absence of 
evidence-based guidelines on corticosteroid treatment ${ }^{[7]}$.

In this thesis, the efficacy of the $\mathrm{IL}-1$ receptor antagonist anakinra was compared to various optimally dosed standard of care treatment options (colchicine, naproxen and prednisone). Anakinra showed non-inferiority to these standard agents in alleviating pain and by similarly improving symptoms associated with acute gout flares after four days (Chapter 2). We believe that these results provide a relevant benchmark for efficacy of anakinra in daily clinical practice and further support the validity of IL-1 blockade in treating acute gout flares.

The finding that anakinra is an effective IL-1 inhibitor for treating acute gout flares may have several implications for current gout management. Firstly, anakinra may be a viable, cheaper alternative to canakinumab, which is presently the only IL-1 blocker registered in Europe. At first sight, it would seem that a head-to-head comparative effectiveness trial of these IL-1 inhibitors could be useful. However, such a trial might be infeasible due to the high treatment cost of canakinumab ( $€ 12,000 /$ treatment in the Netherlands), and to a lesser extent anakinra ( €170/treatment in the Netherlands). Moreover, a large number of participants would likely be required in order to differentiate the effects of two treatments with a similar mechanism of action. More importantly, just considering the difference in treatment costs, reveals that canakinumab cannot be cost-effective compared to anakinra in the Netherlands. That is, even when the difference in treatment effects would be extreme in favor of canakinumab (let's say 0.99 qualityadjusted life weeks), and adopting the maximum willingness-to-pay threshold of $€ 1538$ per week in the Netherlands (€80,000/52 weeks), the incremental cost-effectiveness ratio of canakinumab versus anakinra would be $€ 11,949$ per extra quality-adjusted life week ([€12,000-€170]/0.99). This is far above the cost-effectiveness ceiling in The Netherlands, which means that anakinra would be cost-effective over canakinumab even if unrealistically large health benefits of canakinumab are assumed.

Secondly, the placement of IL-1 inhibitors in clinical practice for managing acute gout flares might be reconsidered. Management guidelines for gout currently recommend treatment with an IL-1 inhibitor for acute gout flares as a second-line option, when standard of care options are not tolerated or ineffective ${ }^{[6,8]}$. For these patients, only canakinumab is currently available when patients have $\geq 3$ gout attacks per year. In particular for patients who cannot be treated with conventional therapies, but who also do not qualify for treatment with canakinumab, anakinra may pose as an alternative treatment option. By contrast, the suitability of anakinra as a first-line agent for treating acute gout flares does seem questionable in light of the findings in this thesis. First of all, 
from a health economic perspective, anakinra had a unfavorable cost and benefit profile compared to first-line anti-inflammatory agents, colchicine, naproxen and prednisone (Chapter 8), which was primarily driven by its higher treatment costs. In addition, for the current conventional first-line treatment agents - colchicine, NSAIDS and corticosteroids - safety profiles have been well documented, while for anakinra little information is still available regarding its safety profile in gout. Thus far, its safety in gout has only been described in this thesis (Chapter 2), various observational studies or an open-label study ${ }^{[9-23]}$. Moreover, treatment with anakinra is associated with increased risk of infections, based upon observed data on infection rates in long-term use of anakinra in patients with rheumatoid arthritis and Still's disease, for which it is currently registered. Whether an increased risk of infections exists with short-term use of anakinra in gout, as applied in the study done in this thesis, has to be further explored, but we may expect it to be lower. Only one patient treated with anakinra reported a respiratory tract infection (severe cold), which suggests that treatment with anakinra is not accompanied with considerable adverse reactions in treating acute gout flares (Chapter 2). However, the study was not powered for safety analysis and more studies are needed to establish the safety profile anakinra in gout.

Finally, besides the application of IL-1 inhibitors in managing acute gout flares, various studies have suggested they might be effective in preventing gout flares during initiation of urate lowering therapy (ULT) ${ }^{[24-28]}$. Currently, prophylaxis when starting ULT is recommended with colchicine and NSAIDs, intended to suppress the triggering of a new flare that may occur due to the dispersion of serum urate (SUA) resulting from the mobility and dissolution of monosodium urate (MSU) crystals ${ }^{[6]}$. Also in this thesis, it was shown that prophylaxis with colchicine and NSAID was protective of gout flare recurrences in patients starting ULT (Chapter 3). However, in selective patients with comorbidities or intolerances to these conventional agents, flare prophylaxis becomes difficult, possibly also contributing to the low ULT adherence rates seen in patients with gout (Chapter 5) ${ }^{[29]}$. There is evidence that the IL-1 inhibitors canakinumab and rilonacept were both successful in gout flare prevention when starting ULT when compared to colchicine and placebo, respectively ${ }^{[24-28]}$. Considering the efficacy of anakinra in the treatment of acute gout flares, the potential added value of anakinra as prophylaxis during initiation of ULT may also be considered. However, the half-life of anakinra ( 4-6 hours) is much shorter than canakinumab ( 26 days) and rilonacept ( 8.6 days). Prophylaxis with anakinra could therefore be costly if daily treatment were to be required to suppress eliciting of gout flares when starting ULT. In addition, daily subcutaneous injections with anakinra could, in time, lead to injection site reactions, and may be associated with increased risk of infections. The efficacy of anakinra in preventing gout flare recurrence 
when starting ULT would need to be studied, but also cost-effectiveness analyses comparing conventional agents and anakinra would be required to determine its added value as a prophylaxis agent in gout management.

\section{Treat to target (T2T) in gout}

In the 1960's with the discovery of the presence of MSU crystals in the synovial fluid of joints, the role of hyperuricemia in the development of gout became more evident. Since then, the importance of lowering SUA levels below the threshold of MSU crystal formation (<6.8 mg/dL) with ULT for inducing crystal dissolution has become clear. Nowadays, the concept of a T2T strategy to achieve predefined SUA targets below this saturation point is increasingly gaining interest, and is now the recommended approach in various international guidelines ${ }^{[6,30]}$. However, the evidence supporting this practice in gout is slim ${ }^{[31,32]}$. The findings from the clinical audit described in this thesis, where realworld gout patients were managed according to the principles of T2T in lowering SUA levels with ULT, is one the first studies to support the concept of T2T in gout (Chapter 4). Just over $80 \%$ of the patients met the European League Against Rheumatism recommended SUA target of $<360 \mu \mathrm{mol} / \mathrm{L}$ (6 mg/dL), using currently available ULT. This percentage was generally higher than those previously reported in observational studies, in which patients were not specifically treated according to a T2T approach (range = 21 to $77 \%$ ) [33-38]. However, it must be noted, that comparison among these studies is difficult considering their observational nature, and differences in patients described and management strategies applied, including frequency of SUA level monitoring visits, medication adherence, dosages and type of ULT used and degree of ULT dose titration. Randomized controlled trials would need to be carried out to provide high quality evidence on the added value of a T2T strategy in gout populations. Such evidence will in addition support the recommendations currently made by gout guidelines that advocate a T2T strategy in meeting SUA targets with ULT in patients with gout and hyperuricemia ${ }^{[6,30]}$. Possibly, randomized controlled trials on this topic have until now been considered irrelevant in gout as the concept of T2T has been intensively investigated in patients with rheumatoid arthritis, consistently showing beneficial results, and the effectiveness of this concept is therefore widely considered self-evident in rheumatology ${ }^{[39]}$. However, in other relevant disciplines, different treatment paradigms are still being proposed. For example, the most recent American College of Physicians guideline endorse the 'treat to avoid symptoms' (TTAS) strategy ${ }^{[40]}$. In contrast to the proactive T2T approach, involving monitoring of SUA levels and making ULT (dose) adjustments to achieve SUA targets in patients on ULT, a TTAS approach encompasses ULT treatment focused on avoiding symptoms (e.g. gout flares) over time ${ }^{[31]}$. The latter can be seen as a more reactive approach, in which ULT treatment adjustments are made in response to the occurrence 
of gout flares or other symptoms, and does not necessarily involve monitoring of SUA levels over time and aiming for a SUA target. Like the T2T approach, the TTAS strategy is currently not supported by solid evidence ${ }^{[40]}$. Hence, the questions remains which of these strategies, T2T or TTAS, is most beneficial for gout patients? From an ethical point of view it may be argued that a TTAS strategy may expose patients to unnecessary risks by reacting upon symptoms and providing them with repeated treatment for recurrent acute gout flares or with courses of ULT. Also, using ULT has shown to decrease the risk of cardiovascular disease and renal impairment through the lowering of SUA levels in (gout) patients having these (comorbid) conditions ${ }^{[41,42]}$. This may not be achieved in a TTAS strategy when ULT may not be given consistently and SUA levels remain elevated. Alternatively, aiming for a therapeutic target as part of a T2T strategy, allows the caregiver to make adjustments in ULT dosages (i.e. up titration of ULT), switching of ULT, or combining ULT in meeting the predefined SUA targets. If not done with caution, being fixed on meeting the SUA target may expose patients to overtreatment with ULT, potentially associated with the occurrence of adverse reactions. Importantly, the choice of the strategy may ultimately depend on the preferences of patients, and therefore patients should be actively involved in choices pertaining to their ULT treatment, contributing to shared-decision making and patient participation. Nevertheless, as guidelines among gout caregivers are proposing different management strategies for long-term ULT, randomized controlled trials may be useful to investigate and compare the risks and benefits of these concepts to stimulate consensus ${ }^{[43-47]}$. Besides consensus on the added value of a T2T strategy in gout, a second step might then be to investigate differences in treatment strategies embedded within a T2T strategy for lowering SUA levels with ULT. For example, sequential monotherapy compared to a strategy allowing combination of ULT ${ }^{[48]}$.

\section{Medication adherence and persistence}

Adherence to long-term ULT among patients with gout and hyperuricemia is necessary for lowering and maintaining SUA levels at a level that dissolution of MSU crystals occurs, contributing to achieving optimal patient outcomes. In the long-term, this may also lead to lower costs of illness and healthcare expenditures, dampening the burden of disease on society ${ }^{[49]}$. Although previous studies in other countries already showed long-term ULT compliance and persistence to be suboptimal, findings from this thesis reveal that this is also an issue among patients receiving ULT for their gout and hyperuricemia in the Netherlands (Chapter 5) ${ }^{[29]}$.

Findings from this thesis showed that patients were most likely to stop with their ULT within the first three months following ULT initiation (Chapter 5). While the reasons for 
treatment cessation and poor adherence were not studied in this thesis, literature suggests that poor medication adherence is a multi-dimensional problem ${ }^{[50]}$. Also in gout, barriers may, for example, be related to the disease (e.g. asymptomatic periods of hyperuricemia), treatment (e.g. adverse reactions), patient or physician (e.g. lack of knowledge) ${ }^{[51]}$. To overcome such barriers, various initiatives have been investigated aimed at improving medication adherence and persistence, including nurse-coordinated programs and (phone-based) pharmacy-led interventions aimed at patient education and individual counseling on gout management ${ }^{[52]}$. Also, gout guidelines emphasize the importance of informing gout patients on disease pathology and management ${ }^{[6]}$. The findings in this thesis suggest that any form of interventions aimed at improving ULT adherence and thus patients outcomes is best introduced early ( $\leq 3$ months) in the management of gout and hyperuricemia as many patients discontinued their treatment during this period (Chapter 5). A recent study by Doherty et al. 2018 showed that a research nurse-led program actively engaging and educating patients in gout management, in combination with a T2T strategy, led to $96.7 \%$ of patients still taking their ULT one year post baseline, compared to $46.8 \%$ receiving usual care ${ }^{[32]}$. Possibly, such a nurse-led program could be effective in the Netherlands as well, as also noted by the authors of the paper that suggest that the management principles applied in their study are potentially generalizable to any health professional that manage gout. However, in the Netherlands, research nurses are generally more common in rheumatology clinics compared to clinics of general practitioners where practice-based nurses are situated. Adoption of the program may therefore be more suited for rheumatology clinics. Nevertheless, also in primary care, such programs may be feasible, which may depend on the willingness of the staff or clinic logistics. Other potential interventions might include electronic self-management tools via mobile applications or computers (i.e. eHealth), but also the efforts of multi-disciplinary teams of experts (e.g. nurse, pharmacist, social worker) may pose useful ${ }^{[52,53]}$. Such interventions may need consideration and investigation in the Netherlands as well.

Patients initially prescribed ULT by a general practitioner were more likely to discontinue ULT treatment, compared to those patients initially receiving ULT from a rheumatologist (Chapter 5). This may reflect a difference in management strategies applied among general practitioners and rheumatologists. A previous study has suggested that among primary care providers, gout and hyperuricemia may be insufficiently treated and be perceived as a disease requiring long-term ULT management, but is more focused on treating the acute episodes of gout ${ }^{[54,55]}$. Future studies may need to investigate whether such a difference also exists among general practitioners and rheumatologists in the Netherlands. 


\section{Measuring the patient perspective of disease}

Over the past decades, including the perspective of the patient in gout clinical research has become increasingly important, as opposed to solely measuring biomedical outcomes ${ }^{[56]}$. Patient-reported outcome measures (PROMs) are therefore a much used tool for gathering patient outcomes data. Ideally, PROMs are developed, or validated, in the population of interest using a psychometric framework that helps to determine whether the items of the instrument are related and measure the intended latent variable, and that the items function in accordance with the a priori expectations of the developers. This may also be referred to as internal construct validity ${ }^{[57]}$. By using appropriate psychometric techniques, poorly performing or weak items can be identified and removed thereby improving the internal construct validity of the instrument. For this purpose item response theory (IRT) models as Rasch modelling, or the more traditional factor analysis are available. Findings from this thesis reveal that such techniques are rarely applied in many of the validation studies of the PROMs used in gout clinical studies (Chapter 6). The results of our systematic review further showed that even when they were applied, the analyses were done either poorly or inconsistently. For example, only for the Health Assessment Questionnaire Disability Index (HAQ-DI), Rheumatoid Arthritis Work Instability Scale and Tophus Impact Questionnaire-20, Rasch-modeling was applied. Therefore, at present little is actually known about the internal construct validity of the PROMs commonly used in gout. On the other hand, the external construct validity of PROMs was more commonly reported on in gout, referring to the association of the PROM to other variables according to predefined hypotheses, to determine the extent that the PROM measures the intended construct ${ }^{[57]}$. However, here the quality of the evidence was supported with few methodological adequate studies. Similarly, other psychometric properties of PROMs, such as their reliability, was also weakly supported. Overall, the measurement properties of PROMs currently used in gout clinical research were shown to be poorly supported. These are an important omission from the current literature because using invalid instruments causes measurement bias, while using unreliable instruments leads to imprecise scores, implying larger sample sizes are needed than necessary ${ }^{[58]}$. This may ultimately influence the study feasibility or increase research costs. Considering the above, the findings from this thesis call for two actions. First, for awareness among users of PROMs in gout clinical research on the importance of using psychometrically robust PROMs for measuring gout outcomes. Secondly, to stimulate research on the measurement properties of commonly used PROMs in gout, using methodological sound studies and, in particular, more detailed studies on the internal construct validity of newly developed or validated PROMs.

Besides the comments on the measurement properties of PROMs in gout, this thesis 
also revealed that various PROMs are currently used in gout clinical research for measuring a single outcome (Chapter 6). For example, in acute gout clinical studies, pain is a commonly used primary outcome for determining the effect of treatment on patients. However various different pain measures are currently used in gout clinical trials, making their results difficult to compare, even when focusing on the same outcome. For instance, in the clinical trials done on the IL-1 inhibitors canakinumab and rilonacept, a 0-100 mm Visual Analogue Scale and a 5-point Likert scale were applied as primary outcome measures, respectively ${ }^{[3,4]}$. Alternatively, in the IL-1 trial done in this thesis, a 5-point rating scale was used for measuring pain in patients with acute gout (Chapter 2). Also the Numeric Rating Scale (NRS) was used as a secondary outcome measure in determining patient-reported pain in this study, but also in the study on rilonacept. The lack of standardization of pain outcomes in such trials in gout makes it difficult to synthesis findings of various studies, for example, for the purpose of metaanalysis. Ideally, standardization of definitions and outcomes measured in these trials are established to enhance comparability, including pain, but also for other outcomes such as joint tenderness, joint swelling or treatment response, which are commonly measured in studies of acute gout. However, also beyond the scope of acute gout trials, findings from this thesis showed that when interested in measuring a specific outcome, for example health-related quality of life, various PROMs are used in clinical studies of gout. The multidimensional health status measures as the Short Form-36 item version 2 (SF-36v2), Gout Assessment Questionnaire 2.0, Arthritis Impact Measurement Scales or Medical Outcomes Study 20-item Short Form Health Survey were commonly used, while the HAQ-DI and Health Assessment Questionnaire-II (HAQ-II) were applied interchangeably for measuring physical disability (Chapter 6). Considering all of the above, this thesis calls for a third action where standardization of patient-reported outcomes measured in gout clinical research is promoted, which will enhance comparability among studies. Various studies have already shown that it is possible to link different instruments measuring the same outcome to a common metric. This has recently been applied for various PROMs measuring physical function, including the HAQ-DI, HAQ-II and SF-36 physical function-10 subscale, which are also instruments commonly used in gout clinical studies (Chapter 6) ${ }^{[59]}$. However, the uptake of such initiatives remains limited which in part may be due lack of knowledge on its implementation. Alternatively, standardization of patient outcomes in gout trials can also be obtained by developing standardized PROMs for relevant outcomes.

In that respect, in the current thesis, work was done to further enhance the standardization of measuring patient-reported outcomes data on symptoms of gout flares that are commonly collected in acute gout trials, including joint pain, swelling 
and tenderness. The GAIS was introduced as a useful summary measure of these three single-item patient reports, for measuring gout flare symptom intensity in acute gout clinical studies, validated using psychometric theory according to non-parametric IRT (Chapter 7). Compared to its single-item components, the GAIS was more reliable and responsive to change, showing similar responsiveness to the NRS pain, which is usually the most responsive instrument in acute gout trials. With the development and validation of the GAIS in this thesis, developers of acute gout clinical trials are invited to use the GAIS as an outcome measure, in the hope to standardize measures and enhance the comparability of outcomes between studies. Also, its adoption in clinical trials implies smaller sample sizes are needed to demonstrate treatment effects, as measurement error is reduced in the combined score, increasing the reliability. As such, the GAIS may have several implications for researchers and developers of acute gout clinical studies, besides allowing for a more comprehensive assessment of gout flare symptoms. However, it must be noted that aspects related to its external construct validity remain unclear, and it would still need further validation in other subpopulations of gout patients (i.e. severe gout). Ideally, the GAIS is also further validated using parametric IRT models such as the Rasch model. In this thesis, the non-parametric IRT Mokken Scale Analysis was used for validating the GAIS. By doing so it was shown that patients can be rank-ordered based on the intensity of their gout flare symptoms by which a higher total score implies higher gout attack symptom intensity. With the limits of an ordinal scale, measuring changes over time is difficult. Therefore, future studies having access to more data are invited to determine whether the GAIS can be considered a quantitative scale using, for example, Rasch analysis.

\section{OVERALL CONCLUSIONS}

From a medical perspective, our findings suggest that for the management of acute gout flares, the IL-1 inhibitor anakinra is an efficacious treatment, showing non-inferiority to conventional treatments in reducing pain and by similarly improving other gout-related symptoms in gout patients, further supporting the validity of the role of IL-1 in gout. We believe anakinra is of added value for managing acute gout flares as a potent second-line treatment, in those patients who cannot be treated with conventional therapies due to unresponsiveness, intolerances or comorbidities, also serving as a potential viable and cheaper alternative to the IL-1 inhibiting agent, canakinumab.

Furthermore, the long-term management of gout and hyperuricemia was shown to be suboptimal in terms of ULT medication adherence in the Netherlands, but also on the 
frequency of gout flare reoccurrences observed in patients starting ULT. For improving gout outcomes, it might be necessary to stimulate ULT adherence early on in the management of gout and hyperuricemia, possibly with adherence interventions such as nurse-coordinated programs. In addition, a T2T strategy in lowering SUA levels with ULT seems promising in reaching SUA targets in gout patients. However, it seems important that the effectiveness of a T2T strategy in gout becomes supported with high-quality evidence, i.e. randomized controlled trials, to clarify and reach consensus on the best long-term managing practices in lowering SUA levels with ULT in patients with gout and hyperuricemia.

Finally, this thesis showed that more evidence on the measurement properties of commonly used PROMs in gout clinical research should become available, based on modern psychometric techniques and methodological sound studies. Also, the GAIS may be used in acute gout trials for determining gout flare symptom intensity. Its adoption will ideally stimulate standardization of outcome measures used in acute gout trials, which will enhance comparison of patient-reported outcomes across these studies. 


\section{REFERENCES}

1. So A, Dumusc A, Nasi S. The role of IL-1 in gout: from bench to bedside. Rheumatology. 2018;57:i12-9.

2. So A, De Meulemeester M, Pikhlak A, Yücel AE, Richard D, Murphy V, et al. Canakinumab for the treatment of acute flares in difficult-to-treat gouty arthritis: Results of a multicenter, phase II, dose-ranging study. Arthritis Rheum. 2010 Jun 8;62(10):3064-76.

3. Schlesinger N, Alten RE, Bardin T, Schumacher HR, Bloch M, Gimona A, et al. Canakinumab for acute gouty arthritis in patients with limited treatment options: Results from two randomised, multicentre, activecontrolled, double-blind trials and their initial extensions. Ann Rheum Dis. 2012;71(11):1839-48.

4. Terkeltaub RA, Schumacher HR, Carter JD, Baraf HSB, Evans RR, Wang J, et al. Rilonacept in the treatment of acute gouty arthritis: a randomized, controlled clinical trial using indomethacin as the active comparator. Arthritis Res Ther. 2013;15:R25.

5. Khanna PP, Gladue HS, Singh MK, FitzGerald JD, Bae S, Prakash S, et al. Treatment of acute gout: A systematic review. Semin Arthritis Rheum. 2014 Aug;44(1):31-8.

6. Richette P, Doherty M, Pascual E, Barskova V, Becce F, Castañeda-Sanabria J, et al. 2016 updated EULAR evidence-based recommendations for the management of gout. Ann Rheum Dis. 2017;76(1):29-42.

7. Neogi T. Interleukin-1 antagonism in acute gout: Is targeting a single cytokine the answer? Arthritis Rheum. 2010;62(10):2845-9.

8. Khanna D, Khanna PP, Fitzgerald JD, Singh MK, Bae S, Neogi T, et al. 2012 American college of rheumatology guidelines for management of gout. part 2: Therapy and antiinflammatory prophylaxis of acute gouty arthritis. Arthritis Care Res. 2012;64(10):1447-61.

9. Ottaviani S, Moltó A, Ea H-K, Neveu S, Gill G, Brunier L, et al. Efficacy of anakinra in gouty arthritis: a retrospective study of 40 cases. Arthritis Res Ther. 2013;15(5):R123.

10. So A, De Smedt T, Revaz S, Tschopp J. A pilot study of IL-1 inhibition by anakinra in acute gout. Arthritis Res Ther. 2007;9(2):R28.

11. Avram A, Duarte C, José Santos M, Papagoras C, Ritis K, Scarpioni R, et al. Identifying Patient Candidates for IL-1 Inhibition: Lessons From Real-World Cases. Jt Bone Spine 82. 2015; eS17-eS29.

12. Ottaviani S, Dieudé P. Tuberculous Cervical Lymphadenitis in a Gouty Patient Treated With Anakinra. J Clin Rheumatol. 2015;21(4).

13. Van Wabeke J, Dhondt E, Peene I, Piette Y. Anakinra in resistant gout: a case report. Acta Clin Belg. $2016 ; 72(4): 293-5$.

14. Palma C, Topping T, Tabechian D. Anakinra is effective and well tolerated in medically complex patients including transplant recipients with gout. In: ACR: Metabolic and Crystal Arthropathies - Poster I: Clinical Practice. 2016.

15. Ghosh P, Cho M, Rawat G, Simkin PA, Gardner GC. Treatment of acute gouty arthritis in complex hospitalized patients with anakinra. Arthritis Care Res. 2013;65(8):1381-4.

16. Vitale A, Cantarini L, Rigante D, Bardelli M, Galeazzi M. Anakinra treatment in patients with gout and type 2 diabetes. Clin Rheumatol. 2015;34(5):981-4. 
17. Chen K, Fields T, Mancuso CA, Bass AR, Vasanth L. Anakinra's Efficacy is Variable in Refractory Gout: Report of Ten Cases. Semin Arthritis Rheum. 2010;40(3):210-4.

18. Thueringer JT, Doll NK, Gertner E. Anakinra for the treatment of acute severe gout in critically ill patients. Semin Arthritis Rheum. 2015;45(1):81-5.

19. Direz G, Noel N, Guyot C, Toupance O, Salmon J-H, Eschard J-P. Efficacy but side effects of anakinra therapy for chronic refractory gout in a renal transplant recipient with preterminal chronic renal failure. Jt Bone Spine. 2012;79(6):631.

20. Nocturne G, Ora J, Ea H-K, Lioté F. Influenza A H1N1 and anakinra exposure in a patient with gout. Jt Bone Spine. 2010;77(4):369-7.

21. O'Brien KL, Pereira SE, Wagner J, Shadman M, Hendrie P, Nelson K, et al. Transfusion-associated graftversus-host disease in a liver transplant recipient: An unusual presentation and review of the literature. Transfusion. 2013;53(1):174-80.

22. Singh D, Huston KK. IL-1 Inhibition With Anakinra in a Patient With Refractory Gout. J Clin Rheumatol. 2009:15(7):366

23. Aouba A, Deshayes S, Frenzel L, Decottignies A, Pressiat C, Bienvenu B, et al. Efficacy of anakinra for various types of crystal-induced arthritis in complex hospitalized patients: a case series and review of the literature. Mediators Inflamm. 2015;2015:7.

24. Schlesinger N, Mysler E, Lin H-Y, De Meulemeester M, Rovensky J, Arulmani U, et al. Canakinumab reduces the risk of acute gouty arthritis flares during initiation of allopurinol treatment: results of a double-blind, randomised study. Ann Rheum Dis. 2011;70(7):1264-71.

25. Mitha E, Ralph Schumacher H, Fouche L, Luo SF, Weinstein SP, Yancopoulos GD, et al. Rilonacept for gout flare prevention during initiation of uric acid-lowering therapy: Results from the PRESURGE-2 international, phase 3, randomized, placebo-controlled trial. Rheumatol (United Kingdom). 2013;52(7):1285-92.

26. Sundy JS, Schumacher HR, Kivitz A, Weinstein SP, Wu R, King-Davis S, et al. Rilonacept for gout flare prevention in patients receiving uric acid-lowering therapy: Results of RESURGE, a phase III, international safety study. J Rheumatol. 2014;41(8):1703-11.

27. Schumacher HR, Evans RR, Saag KG, Clower J, Jennings W, Weinstein SP, et al. Rilonacept (interleukin-1 trap) for prevention of gout flares during initiation of uric acid-lowering therapy: Results from a phase III randomized, double-blind, placebo-controlled, confirmatory efficacy study. Arthritis Care Res. 2012;64(10):1462-70.

28. Schumacher HR, Sundy JS, Terkeltaub R, Knapp HR, Mellis SJ, Stahl N, et al. Rilonacept (interleukin-1 trap) in the prevention of acute gout flares during initiation of urate-lowering therapy: Results of a phase II randomized, double-blind, placebo-controlled trial. Arthritis Rheum. 2012;64(3):876-84.

29. Scheepers LEJM, van Onna M, Stehouwer CDA, Singh JA, Arts ICW, Boonen A. Medication adherence among patients with gout: A systematic review and meta-analysis. Semin Arthritis Rheum. 2018;47(5):689-702.

30. Khanna D, Fitzgerald JD, Khanna PP, Bae S, Singh MK, Neogi T, et al. 2012 American college of rheumatology guidelines for management of gout. part 1: Systematic nonpharmacologic and pharmacologic therapeutic approaches to hyperuricemia. Arthritis Care Res. 2012;64(10):1431-46. 
31. Perez-Ruiz F, Moreno-Lledó A, Urionagüena I, Dickson AJ. Treat to target in gout. Rheumatology. 2018;57(1):i20-6.

32. Doherty M, Jenkins W, Richardson H, Sarmanova A, Abhishek A, Ashton D, et al. Efficacy and cost-effectiveness of nurse-led care involving education and engagement of patients and a treat-to-target urate-lowering strategy versus usual care for gout: a randomised controlled trial. Lancet. 2018;392(10156):1403-12.

33. Cottrell E, Crabtree V, Edwards JJ, Roddy E. Improvement in the management of gout is vital and overdue: an audit from a UK primary care medical practice. BMC Fam Pract. 2013;14(1):170.

34. Arroll B, Bennett M, Dalbeth N, Hettiarachchi D, Cribben B, Shelling G. More allopurinol is needed to get gout patients $<0.36 \mathrm{mmol} / \mathrm{L}$ : a gout audit in the form of a before-after trial. J Prim Health Care. 2009:1(4):315-8.

35. Wall GC, Koenigsfeld CF, Hegge KA, Bottenberg MM. Adherence to treatment guidelines in two primary care populations with gout. Rheumatol Int. 2010;30(6):749-53

36. Sarawate CA, Patel PA, Schumacher HR, Yang W, Brewer KK, Bakst AW. Serum Urate Levels and Gout Flares Analysis from managed care data. J Clin Rheumatol. 2006;12(2):61-5.

37. Roddy E, Zhang W, Doherty M. Concordance of the management of chronic gout in a UK primary-care population with the EULAR gout recommendations. Ann Rheum Dis. 2007;66(10):1311-5.

38. Khanna P, Khanna D, Storgard C, Baumgartner S, Morlock R. A world of hurt: failure to achieve treatment goals in patients with gout requires a paradigm shift. Postgrad Med. 2016;128(1):34-40.

39. Smolen JS, Breedveld FC, Burmester GR, Bykerk V, Dougados M, Emery P, et al. Treating rheumatoid arthritis to target: 2014 update of the recommendations of an international task force. Ann Rheum Dis. $2016 ; 75(1): 3-15$

40. Qaseem A, McLean RM, Starkey M, Forciea MA, Denberg TD, Barry MJ, et al. Management of Acute and Recurrent Gout: A Clinical Practice Guideline From the American College of Physicians. Ann Intern Med. 2017;166:58-98.

41. Volterrani M, lellamo F, Sposato B, Romeo F. Uric acid lowering therapy in cardiovascular diseases. Int $J$ Cardiol. 2016;213:20-2.

42. Levy G, Shi JM, Cheetham CT, Rashid N. Urate-Lowering Therapy in Moderate to Severe Chronic Kidney Disease. Perm J. 2018;22:17-142.

43. Dalbeth N, Bardin T, Doherty M, Lioté F, Richette P, Saag KG, et al. Discordant American College of Physicians and international rheumatology guidelines for gout management: Consensus statement of the Gout, Hyperuricemia and Crystal-Associated Disease Network (G-CAN). Nat Rev Rheumatol. 2017;13(9):561-8.

44. Neogi T, Mikuls TR. To treat or not to treat (to target) in gout. Ann Intern Med. 2017;166(1):71-2.

45. McLean RM. The long and winding road to clinical guidelines on the diagnosis and management of gout. Ann Intern Med. 2017;166(1):73-4.

46. Neogi T, Dalbeth N. Patient education and engagement in treat-to-target gout care. Lancet. 2018;392:137981.

47. Jansen TL, Janssen M. The American College of Physicians and the 2017 guideline for the management of acute and recurrent gout: treat to avoiding symptoms versus treat to target. Clin Rheumatol. 2017;36(11):2399-402. 
48. Jansen TL. Treat to target in gout by combining two modes of action: Targeting serum uric acid. Rheumatol (United Kingdom). 2014;53(12):2131-3.

49. Sabaté E. Adherence to Long-Term Therapies: Evidence for Action. World Health Organization. Geneva, Switzerland; 2003.

50. Brown MT, Bussell JK. Medication adherence: WHO cares? Mayo Clin Proc. 2011;86(4):304-14.

51. Perez-Ruiz F, Desideri G. Improving adherence to gout therapy: An expert review. Ther Clin Risk Manag. 2018;14:793-802.

52. Fields TR, Batterman A. How Can We Improve Disease Education in People with Gout? Curr Rheumatol Rep. 2018;20(3):12.

53. Day RO, Frensham LJ, Nguyen AD, Baysari MT, Aung E, Lau AYS, et al. Effectiveness of an electronic patientcentred self-management tool for gout sufferers: A cluster randomised controlled trail protocol. BMJ Open. 2017;7(10):1-12.

54. Harrold LR, Mazor KM, Negron A, Ogarek J, Firneno C, Yood RA. Primary care providers' knowledge, beliefs and treatment practices for gout: Results of a physician questionnaire. Rheumatol (United Kingdom). 2013;52(9):1623-9.

55. Chia FLA. Poorly controlled gout: Who is doing poorly? Singapore Med J. 2016;57(8):412-4.

56. Mercieca-Bebber R, King MT, Calvert MJ, Stockler MR, Friedlander M. The importance of patient-reported outcomes in clinical trials and strategies for future optimization. Patient Relat Outcome Meas. 2018;9:353-67.

57. Grimm KJ, Widaman KF. Construct validity. In: Cooper H, Camic PM, Long DL, Panter AT, Rindskopf D, Sher $\mathrm{KJ}$, editors. APA handbook of research methods in psychology, Vol 1: Foundations, planning, measures, and psychometrics. Washington, DC, US: American Psychological Association; 2012. p. 621-42.

58. Kimberlin CL, Winterstein AG. Validity and reliability of measurement instruments used in research. Am J Heal Pharm. 2008;65(23):2276-84.

59. Oude Voshaar MAH, Vonkeman HE, Courvoisier D, Finckh A, Gossec L, Leung YY, et al. Towards standardized patient reported physical function outcome reporting: linking ten commonly used questionnaires to a common metric. Qual Life Res. 2019;28(1):187-97. 
<smiles>CO[C@H]1O[C@H](O)[C@H](O)O1</smiles> 
Appendices 
Dutch summary I

Nederlandse samenvatting 


\section{JICHT: PATIËNTEN, GENEESKUNDE EN DE MAATSCHAPPIJ}

Jicht is een veelvoorkomende inflammatoire gewrichtsaandoening behorend tot de reumatische aandoeningen. Het wordt veroorzaakt door neergeslagen urinezuurkristallen in de synoviaal vloeistof van gewrichten als gevolg van langdurige hyperurikemie. Het (plotseling) optreden van een dergelijke ontstekingsreactie wordt vaak een acute jichtaanval genoemd en is gekenmerkt door symptomen van pijn, zwelling, roodheid en gewrichtsgevoeligheid. Herhaalde aanvallen van acute jicht kunnen leiden tot verergering van de ziekte en ophoping van urinezuurkristallen in gewrichten en weefsel, ook wel (chronische) tofeuze jicht (jichtknobbels) genoemd. Over het algemeen kan jicht leiden tot een verminderde kwaliteit van leven en verminderd fysiek functioneren.

In dit proefschrift worden verschillende onderzoeken gepresenteerd op het gebied van jicht en hyperurikemie. Het proefschrift is opgedeeld in drie delen. Het eerste gedeelte bevat studies die zich richten op de klinische behandelingen van jicht en hyperurikemie. De studies die in het tweede deel van dit proefschrift worden beschreven richten zich op het meten van de gevolgen van jicht vanuit het perspectief van de patiënt met behulp van patiënt gerapporteerde uitkomstmaten (PROMs). In het derde gedeelte wordt ingegaan op de maatschappelijke ziektelast van jicht en hyperurikemie in termen van de kosten en effectiviteit van de verschillende behandelingen voor deze ziektes.

\section{Deel I: Klinische behandeling van jicht en hyperurikemie}

Een acute jichtaanval is goed te behandelen met een van de drie standaard behandelingen: colchicine, niet-steroïde anti-inflammatoire geneesmiddelen of corticosteroïden. Echter, veel patiënten met jicht hebben comorbiditeiten die behandeling met deze middelen bemoeilijkt wegens contra-indicaties. Ook wordt door de aanwezigheid van bijwerkingen of intoleranties in sommige gevallen de behandeling van een acute jichtaanval bemoeilijkt. Voor patiënten die niet behandeld kunnen worden met een van de standaardbehandelingen, bevelen jicht richtlijnen een behandeling met een interleukine-1 (IL-1) remmer aan. Tot op heden is in Europa alleen tegen hoge kosten enkel de IL-remmer canakinumab beschikbaar voor patiënten met $\geq 3$ aanvallen per jaar en die niet behandeld kunnen worden met standaardzorg. Anakinra is een alternatieve, goedkopere, IL-1 remmer. Uit verschillende observationele studies is al gebleken dat anakinra mogelijk effectief zou kunnen zijn voor het behandelen van acute jicht. Echter, tot op heden zijn er nog geen gerandomiseerde klinische studies geweest om dit te bevestigen. De studie in Hoofdstuk 2 van deze thesis had als doel te bepalen of een 5 -daagse behandeling van een dagelijkse $100 \mathrm{mg}$ subcutane injectie van de IL-1 
receptorantagonist anakinra, een effectief en veilig behandelingsalternatief zou zijn voor de behandeling van acute jichtaanvallen. Om dit te bepalen is er in Nederland een multicenter, dubbelblind, dubbel dummy, gerandomiseerd, actief-gecontroleerd, non-inferiority (NI) (niet inferieur aan) studie ontworpen en uitgevoerd, waarbij anakinra werd vergeleken met standaardzorg (colchicine, naproxen of prednison). In totaal zijn 88 mensen geïncludeerd, waarvan 45 en 43 patiënten behandeld zijn met respectievelijk standaardzorg en anakinra. Anakinra was non-inferior aan standaardzorg in het verminderen van pijn klachten in patiënten met een acute jichtaanval. Ook daalden in beide behandelingsgroepen in gelijke mate van dag één tot vijf andere jichtgerelateerde symptomen zoals gewrichtsgevoeligheid en zwelling. De resultaten van deze studie suggereren dat anakinra een effectieve IL-1 remmer is voor de behandeling van acute jichtaanvallen.

Het verlagen van serum urinezuur (SUZ) spiegels bij patiënten met jicht vormt een belangrijk onderdeel van het behandelingsplan. Dit is onder andere omdat verlaagde SUZ spiegels tot onder de drempelwaarde van urinezuur kristalformatie in verband is gebracht met een verminderd risico op het krijgen van jichtaanval recidieven. Voor het verlagen van de SUZ spiegels in patiënten met jicht wordt daarom behandeling met urinezuurverlagende therapie (UVT) aanbevolen. Echter, zelfs bij jicht patiënten die behandeld worden met UVT zijn jicht recidieven veelvoorkomend, met name in de periode direct na het starten van UVT. In de studie in Hoofdstuk 3 werd de prognostische waarde van verschillende klinische, demografische en patiënt-gerapporteerde factoren voor het voorspellen van vroege ( $\leq 3$ maanden) jichtaanval recidieven onderzocht bij patiënten die UVT zijn gestart tijdens een acute jichtaanval $(N=75)$. Binnen de eerste drie maanden had $48 \%(n=36)$ van de patiënten opnieuw een jichtaanval. Resultaten van de binaire multivariate regressie analyse lieten zien dat geen profylaxe bij het starten van UVT (odds ratio (OR) 11.56) en c-reactief proteïne > $30 \mathrm{mg} / \mathrm{L}$ (OR 9.47), onafhankelijke voorspellers waren voor een recidief van een jichtaanval binnen de eerste 3 maanden na baseline.

Momenteel is weinig bekend over de mogelijke toegevoegde waarde van het toepassen van een treat-to-target (T2T) strategie (behandelen naar een vooraf bepaald doel/target) in de klinische praktijk voor het verlagen van SUZ spiegels met UVT in patiënten met jicht. Echter, een T2T strategie bij behandeling met UVT wordt momenteel wel aanbevolen door verschillende jicht richtlijnen voor het verlagen van SUZ spiegels. Hoofstuk 4 presenteert de resultaten van een klinische audit van real-world jichtpatiënten (patiënten zoals gezien in de dagelijkse klinische praktijk) die behandeld werden met beschikbare UVT conform het T2T concept. Voor dat laatste werden de SUZ targets aangehouden $(<360 \mu \mathrm{mol} / \mathrm{L}$ en 
< $300 \mu \mathrm{mol} / \mathrm{L}$ ) van de European League Against Rheumatism. Voor deze retrospectieve studie zijn de medische patiëntendossiers van jicht patiënten uit twee verschillende ziekenhuizen in Nederland onderzocht. De resultaten laten zien dat het SUZ doel van < $360 \mu \mathrm{mol} / \mathrm{L}$ in $83 \%$ van de gevallen werd bereikt in de dagelijkse klinische praktijk met de huidige beschikbare UVT en een behandelingswijze conform de T2T strategie. In meer dan de helft van de patiënten (59\%) werd ook de SUZ target van $<300 \mu \mathrm{mol} / \mathrm{L}$ bereikt. Deze resultaten suggereren dat het concept T2T werkt bij de behandeling van jicht en dat de toepassing van deze strategie in de klinische praktijk kan bijdragen bij het verhogen van het percentage jicht patiënten met SUZ spiegels op target.

Verschillende internationale studies tonen aan dat de therapietrouw van langdurig UVT suboptimaal is bij patiënten met jicht. Tot op heden is onbekend of dit ook geldt voor patiënten met jicht en hyperurikemie in Nederland. In de studie in Hoofdstuk $\mathbf{5}$ is de therapietrouw (compliance en persistence) van patiënten met jicht en hyperurikemie bepaald middels het analyseren van uitgegeven UVT medicatie recepten afkomstig van een landelijk representatieve database. De naleving van voorgeschreven medicatie (compliance) was na één jaar 51.7\%, wat inhoudt dat iets meer dan de helft van de patiënten UVT-dekking had voor $\geq 80 \%$ van de dagen in een jaar. Na een jaar zette $42.7 \%$ van de patiënten de medicatie nog voort (persistence), zonder dat een refillgap (tijdsduur voor het ophalen van herhaalrecepten) van 30 dagen werd overschreden. Toenemende leeftijd, het zijn van een man en wanneer het eerste UVT recept door een reumatoloog werd voorgeschreven waren factoren geassocieerd met een toenemende therapietrouw (zowel compliance als persistence). De resultaten van dit onderzoek suggereren dat de therapietrouw van UVT in Nederland suboptimaal is en niet conform de jichtrichtlijnen die levenslange behandeling met UVT aanbevelen. Toekomstige studies zijn gewenst naar de redenen van stoppen en naar manieren om therapietrouw te verbeteren.

\section{Deel II: Patiënt-gerapporteerde uitkomstmaten (PROMs) in jicht}

PROMs (instrumenten) worden veel gebruikt in studies met jicht om inzicht te krijgen wat de gevolgen van ziekte zijn vanuit de perspectief van de patiënt. Bij het gebruik van PROMs is het belangrijk dat de meeteigenschappen goed onderbouwd zijn zodat de gemeten uitkomsten betrouwbaar zijn. Hoofdstuk 6 presenteert de resultaten van een systematische literatuur studie die gericht was op het identificeren van alle beschikbare PROMs voor jicht en kritisch te beoordelen wat de inhoudsvaliditeit en andere meeteigenschappen (betrouwbaarheid, gevoeligheid voor verandering, construct validiteit, vloer- en plafondeffecten) van deze PROMs zijn. De zoekopdracht resulteerde in 13 PROMs, die bijna alle uitkomstdomeinen omvatten die zijn goedgekeurd 
voor gebruik bij acute en chronische jicht studies. Opmerkelijk was dat voor het meten van patiënt-gerapporteerde gewrichtszwelling en gewrichtsgevoeligheid geen PROMs en voor specifiek jicht slechts drie PROMs beschikbaar waren. Resultaten van deze studie lieten zien dat de subschaal voor fysiek functioneren van de Short Form-36 item versie 2, de enige PROM is waarvoor voldoende bewijs was voor de psychometrische eigenschappen die in deze studie werden onderzocht. Vanuit het bredere perspectief bleek uit dit onderzoek dat voor veel van de PROMs die in klinische studies bij jicht gebruikt worden, de meeteigenschappen nog niet goed ondersteund zijn.

Het doel van de studie in Hoofdstuk 7 was om een gemiddelde score te ontwikkelen die de algehele intensiteit van een jichtaanval weergeeft, de Gout Attack Intensity Score (GAIS), samengesteld uit individuele symptoomscores van patiënt-gerapporteerde gewrichtspijn, zwelling en gevoeligheid, gemeten op een 5-punts antwoordschaal. Uit de resultaten bleek dat de GAIS kan worden geïnterpreteerd als een ordinale schaal, waarbij hogere scores een hogere jichtaanval symptoom intensiteit aangeven. De GAIS bleek betrouwbaar en gevoelig te zijn voor verandering. Ook was de GAIS beter in staat onderscheid te maken tussen mensen die wel en niet reageerde op behandeling, in vergelijking tot de individuele items waaruit het bestaat (pijn, zwelling, gevoeligheid). Resultaten van de studie suggereren dat de GAIS gebruikt kan worden in klinische studies naar jicht voor het bepalen van de intensiteit van een jichtaanval.

\section{Deel III: Maatschappelijke ziektelast van jicht (disease burden of gout)}

Het doel van de studie beschreven in Hoofdstuk 8 was het bepalen van de kosteneffectiviteit van verschillende combinaties van UVT (allopurinol, febuxostat, geen UVT) en anti-inflammatoire behandelingen voor acute jichtaanvallen (colchicine, naproxen, prednison, anakinra). Hiervoor is een gezondheidseconomische evaluatie uitgevoerd op basis van een simulatie op patiëntniveau. Nieuw gediagnosticeerde jicht patiënten, gestart met UVT tijdens een jichtaanval, zijn in het model voor een jaar gevolgd voor het schatten van de kosten en de effectiviteit (uitgedrukt in quality-adjusted life years) van de verschillende behandelingscombinaties voor jicht en hyperurikemie. Het model is de eerste op het gebied van jicht die gelijktijdig de behandelingen van UVT en acute jicht evalueert. In vergelijking met 'Geen UVT' waren zowel allopurinol en febuxostat kosteneffectief, en bij een hogere betalingsbereidheid (willingness-to-pay) (ongeveer > €25.173) was febuxostat kosteneffectief in vergelijking met allopurinol, in combinatie met verschillende ontstekingsremmende middelen. Voor de behandeling van acute jichtaanvallen en onafhankelijk van welke UVT er werd gebruikt, hadden colchicine, naproxen en prednison vergelijkbare resultaten, hoewel naproxen over het algemeen het meest gunstige profiel op kosteneffectiviteit bood. Met name als gevolg 
van de hoge kosten per behandeling was anakinra niet kosteneffectief in vergelijking met standaardzorg, al leverde het wel de hoogste utiliteit .

\section{Algehele conclusies}

Vanuit een medisch perspectief suggereren onze bevindingen dat de IL-1 remmer anakinra een effectieve behandeling is voor het behandelen van acute jichtaanvallen. Anakinra was non-inferior aan standaardzorg in het verminderen van pijn in patiënten met een acute jichtaanval en het verminderde andere jicht-gerelateerde symptomen in gelijke mate als standaardzorg. Dit ondersteunt verder de validiteit van de rol van IL-1 in jicht. Wij geloven dat anakinra van toegevoegde waarde is voor het behandelen van acute jichtaanvallen als een effectieve, tweedelijnsbehandelingsoptie. Met name voor patiënten met jicht die niet met standaardzorg behandeld kunnen worden door intoleranties, comorbiditeiten of niet succesvol reageren op deze behandelingen. Daarnaast zou anakinra mogelijk kunnen dienen als een doelmatig en goedkoper alternatief voor de IL-1 remmer canakinumab.

Verder bleek de langdurige behandeling van jicht en hyperurikemie suboptimaal te zijn, in termen van UVT therapietrouw in Nederland, maar ook kijkend naar de frequentie van terugkerende jichtaanvallen in patiënten die UVT zijn gestart. Voor het verbeteren van de uitkomsten in patiënten met jicht kan het nodig zijn om UVT therapietrouw vroegtijdig te stimuleren bij het behandelen van jicht en hyperurikemie, mogelijk met behulp van interventies gericht op therapietrouw zoals programma's gecoördineerd door verpleegkundigen. Daarnaast bleek een T2T strategie voor het verlagen van SUZ spiegels met UVT in patiënten met jicht veelbelovend in het behalen van de aanbevolen SUZ targets. Het is echter belangrijk dat de effectiviteit van een T2T strategie voor jicht ondersteund wordt met hoogwaardig bewijs, d.w.z. gerandomiseerde studies, om opheldering en consensus te bereiken over de beste, langdurige strategie om patiënten met jicht en hyperurikemie te behandelen voor het verlagen van SUZ spiegels met UVT.

Tot slot toont deze thesis aan dat er meer bewijs over de meeteigenschappen van veelgebruikte PROMs in jicht beschikbaar moet komen, idealiter gebaseerd op moderne psychometrische technieken en methodologisch degelijke studies. Daarnaast kan de GAIS worden gebruikt in studies van acute jicht voor het bepalen van de intensiteit van een jichtaanval. De brede adoptie van de GAIS zou de standaardisatie van uitkomstmaten in acute jicht studies kunnen stimuleren, wat de vergelijking van patiënt-gerapporteerde uitkomsten tussen deze studies zou kunnen verbeteren. 


\section{Acknowledgements I \\ Dankwoord}


De afgelopen viereneenhalf jaar zijn een groot avontuur geweest, waar ik met veel plezier op terugkijk! Ik heb niet alleen veel geleerd over mezelf en het doen van medisch wetenschappelijk onderzoek, maar ben ook ontzettend dankbaar voor alle mensen die ik heb leren kennen, de leuke momenten die we hebben gehad en de hulp die ik gedurende mijn promotietraject heb ontvangen. Ik wil graag een aantal mensen in het bijzonder bedanken.

Beste Mart, Martijn en Harald, ik ben jullie enorm dankbaar voor jullie betrokkenheid bij het tot stand komen van dit proefschrift.

Allereerst wil ik mijn grootste dank betuigen aan mijn copromotor, Martijn. Zonder onze wekelijkse overleggen, onder het genot van een heerlijk kopje koffie, had dit proefschrift er nu niet gelegen. Ik heb grote bewondering voor je gedrevenheid voor de wetenschap, je analytische vermogen en je pragmatische aanpak. Ook heb ik enorm genoten van onze brainstormsessies over hoe we gedurende mijn onderzoek praktische vraagstukken het beste aan konden vliegen. Bijvoorbeeld, hoe moet de medicatie gecodeerd worden? Of, hoe moet de e-CRF eruit komen te zien in Mijn Reumacentrum? Of, hoe gaan we om met de verlopen medicatie? (Ja, dit laatste heeft me wel een flinke hoofdpijn bezorgd, gelukkig zag jij het allemaal nog positief in). Naar mijn mening hebben we het ver geschopt! Bedankt voor je laagdrempelige karakter, benaderbaarheid en de vele gezellige gesprekken tijdens de lunches. Tot slot, wil ik je bedanken voor je geduld; de vele keren dat jij uitleg hebt moeten geven over psychometrie en statistiek. Dit alles heeft mij enorm geholpen. Ik heb veel van je geleerd en hoop dat we nog eens mogen samenwerken in de toekomst!

Daarnaast gaat mijn dank uit naar mijn promotor, Mart van de Laar. Je gedrevenheid voor het vak en onderzoek hebben me gedurende de jaren geïnspireerd. Ondanks je drukke agenda, was je altijd bereid tot overleg om het onderzoek verder te brengen. Ik heb veel van je mogen leren over de klinische praktijk en de obstakels die zich voordoen bij het uitvoeren van medisch onderzoek. Bedankt dat je de grote lijnen goed hebt bewaakt en het vertrouwen wat je me gaf om het maximale uit mezelf te halen en het onderzoek een stapje verder te brengen!

Dan mijn andere copromotor, Harald. Met je kritische en klinische blik heb je een goede bijdrage geleverd aan de kwaliteit van mijn proefschrift. Ik kon je feedback op mijn stukken altijd erg waarderen! Ik wil je bedanken voor je humor, gezelligheid en vooral voor alle hulp en input die je gedurende mijn promotietraject hebt verleend. 
Ik wil graag alle leden van de commissie bedanken, Prof. dr. A. So, Prof. dr. T. Bardin, Prof. dr. R. Westhovens, Prof. dr. E.T. Bohlmeijer en dr. E. Taal, voor de tijd en moeite die is genomen om mijn proefschrift te lezen, de feedback te leveren en plaats te willen nemen in mijn commissie.

In het bijzonder wil ik bedanken mijn paranimf, reismaatje en kamergenoot, Annemieke. Bedankt voor alle gezellige uren die we samen in de trein (met een bakje yoghurt en cruesli, boterham, make-up of een kaasbroodje), en later in jouw auto (met gezellige muziek op de achtergrond) hebben mogen doorbrengen. Ondanks de wervelwind die je soms in de ochtend was, was je altijd optimistisch en vrolijk. Hiervoor ben ik je ontzettend dankbaar; je zorgde er altijd voor dat we de dag begonnen met een lach. Ook wil ik je bedanken voor alle dropjes, koekjes, rijstwafels, ijsjes, chocola en kaasbroodjes die je me gedurende de jaren hebt geschonken. Je leek soms op mijn persoonlijke 'feeder'. Gelukkig kon ik je af en toe ook blij maken met een appeltje ;). Ik heb enorm genoten van alle cursussen die we samen hebben mogen volgen en de goede gesprekken die we over de jaren hebben gehad. Kortom, ik ga je als kamergenoot missen en hoop nog vaak samen koffie en thee te mogen drinken in Deventer, Enschede, Bennekom of Kerkdriel.

Mijn andere paranimf, Teuntje. Lieve Teuntje, de enige echte Bathmen/Demtenaar! Onze treinreisjes naar huis gingen altijd té snel voorbij, we hadden zoveel te bespreken. Wat kon ik het waarderen dat je uit de regio kwam, de beste restaurantjes, kroegjes en regionale evenementen was jij vaak van op de hoogte! Ik heb dan ook enorm genoten van onze get-togethers in Deventer en zelfs al in Bennekom. Ik vind het superleuk dat je mijn paranimf wilde zijn en hoop nog vaak gezellige uitjes en gesprekken te hebben!

Laura, mijn partner in crime als het aankomt op onderzoek doen op het gebied van reumatologie. Wat was ik blij met jouw aanwezigheid de afgelopen jaren. Een van de hoogste punten was misschien wel het samen op congres gaan naar Madrid. Warm weer, een luxehotel en een privé zwembad. Daarnaast ook alle binnenlandse congressen, symposia en cursussen die we samen hebben mogen volgen waren me een waar genoegen. Ik vond het een hele eer om je paranimf te zijn. Bedankt voor je steun, gezelligheid en openhartelijke gesprekken.

Lieve Deniece, wat vond ik het verfrissend om jou als kamergenoot te hebben. De fijne gesprekken over het dagelijkse leven en PhD-life hebben me goed gedaan. Met name de vrijdagen als we als enige twee aan het werk waren in onze vleugel, zijn momenten die me goed bijstaan. Ondanks dat we maar kort kamergenoten waren, heb ik je betrokkenheid enorm gewaardeerd! 
Ik wil alle collega's binnen de vakgroep PGT bedanken voor jullie hulp gedurende mijn promotietraject. In het bijzonder Peter ten Klooster voor je betrokkenheid bij verschillende projecten die nu onderdeel uitmaken van mijn proefschrift. Ook Marieke Smellink-Kleisman en Daniëlle Boelen voor jullie ondersteuning bij alle regel-dingetjes en al mijn mede-promovendi binnen de vakgroep voor de gezellige gesprekken en leuke Rookie-overleggen en uitjes.

In het bijzonder gaat mijn dank uit naar alle verpleegkundige, artsen, AIO's, doktersassistenten en secretaressen die hebben bijgedragen aan het ATTACG onderzoek in de volgende ziekenhuizen: Viecuri Medisch Centrum, Rijnstate Ziekenhuis, Medisch Spectrum Twente, ZiekenhuisGroep Twente, Maastricht Universitair Medisch Centrum+, Maasstad ziekenhuis, Medisch Centrum Leeuwarden, Röpcke-zweers ziekenhuis en Bernhoven ziekenhuis. Het vele werk wat jullie hebben verricht om het onderzoek tot een succes te maken is onbeschrijfelijk. Ook alle ziekenhuisapothekers en personeel die hebben geholpen, en alle patiënten die bereid waren tot deelname aan het onderzoek, bedankt! Bastiaan Nuijen, bedankt voor de vele medicatie pakketjes die jij herhaaldelijk gereed hebt gemaakt.

Lieve Riette, Jolanda, Jacqueline, Mirjam en Francis, wat heb ik ontzettend genoten van alle gezellige lunches en met name de lekkere cappuccino's. Ik kom met alle liefde nog eens naar Enschede om deze traditie voort te zetten. Door jullie aanwezigheid heb ik met veel plezier op het MST gewerkt! Lieve Mirjam, al je inzet, je KitKat taart recept en research kennis, dit onderzoek was zonder jou niet van de grond gekomen; ontzettend bedankt! Jolanda, je bent een GCP-held! Wat heb ik veel van je geleerd en wat vond ik het fijn om je te kunnen raadplegen als ik het zelf even niet zeker wist! Francis en Marieke, bedankt voor de gezelligheid in Madrid tijdens de EULAR. Heleen en Ilse, bedankt voor de gezellige gesprekken op de donderdagen in het MST.

Daarnaast wil ik al mijn vrienden, verspreid over heel Nederland, bedanken voor alle afleiding en gezelligheid die jullie me gedurende deze jaren in Deventer en Enschede hebben geboden en de interesse die jullie hebben getoond in mijn onderzoek. Ik ben ontzettend dankbaar dat ik zulke lieve vrienden om me heen heb. Lieve Richelle K., bedankt voor je steun en gezellige borrels, uitjes en etentjes gedurende deze jaren. Ik ben ontzettend blij met jou als vriendin en ceremoniemeester, je bent een topper! Lieve Jannicke, Moniek C., Michèlle en Caroline, onze vertrouwde voedingsmiepen groep. Ik vond het heerlijk om met jullie te kunnen sparren en reisjes mee te pakken. Lieve Suzan, ik vind het superleuk dat we nu zo dicht bij elkaar wonen. De boswandelingen van het afgelopen half jaar hebben me goed gedaan. Lieve Moniek L., ik heb enorm 
genoten van onze afspraakjes in Nijmegen, Deventer, Zoetermeer, Rosmalen, Utrecht, de locatie maakte niet uit, met jou was het altijd leuk! Lieve Stefanie, Anouk, Richelle, Danielle, Floor en Fleur en jullie partners, ik heb enorm genoten van al onze etentjes en feestjes de afgelopen jaren! Lieve Deja-vu, bedankt voor jullie bezoekjes aan Deventer, weekendjes-weg, feestjes, reisjes en etentjes. Ik heb ontzettend genoten van jullie bezoek aan Deventer voor de IJsselloop, ik wist niet dat er zoveel hardloop-liefhebbers waren in onze club! Lieve Emmy, wat vind ik het fijn om jou als vriendin te hebben. Je bent altijd wel in voor een lekker hapje eten of een dansje op Nederlandstalige hits! Lieve Richelle V., wat is het heerlijk om met jou te borrelen en te kletsen, je luisterende oor heb ik ontzettende gewaardeerd de afgelopen jaren. Je wist me altijd van positieve energie te voorzien! Lieve Jeanne, Janneke, en Lydia, mijn vriendinnetjes uit Wijchen. Jullie ken ik inmiddels het langst. Ik vind het ontzettend leuk dat we nog steeds zulk goed contact hebben en dat we elkaar zo goed kennen. Lieve Thymos, bedankt voor jullie interesse en betrokkenheid. Lieve huisgenootjes van de Erwt, bedankt voor alle gezelligheid!

Een speciaal woord van dank gaat uit naar mijn familie. Lieve mam en pap, jullie onvoorwaardelijke steun gedurende al mijn jaren als leerling op de basis en middelbare school (zowel in Nederland als Amerika), student op de universiteit en de afgelopen jaren als PhD-student, heb ik enorm gewaardeerd. Jullie hebben me altijd gesteund om een opleiding te volgen, iets te doen wat ik leuk vind en het beste uit mezelf te halen. Jullie doorzettingsvermogen zie ik als voorbeeld. Mijn oudere zussen. Lieve Lindsey en Mandy, jullie zorgen altijd goed voor jullie kleine zusje. Dank voor al jullie steun, betrokkenheid en de voorbeelden die jullie hebben gegeven om altijd je droom te blijven volgen. Lieve Lex, Koen en de kleinste familieleden Anna, Dex en Julia; zonder jullie gezelligheid, goede gesprekken en goede afleidingen had dit boekje er vandaag niet gelegen! Bedankt.

Ook mijn (soon to be) schoonfamilie wil ik ontzettend bedanken! Anja en Henk, jullie staan altijd klaar om te helpen en jullie mentale, maar ook fysieke steun (tijdens de verschillende verhuizingen) stel ik erg op prijs. Daan, nu zijn we beide klaar met promoveren! Bedankt voor je nuchtere blik en relativeringsvermogen gedurende de afgelopen jaren ;-). Nel, Ine, Richard en Jonas, jullie aanwezigheid is altijd zo verfrissend. Ik waardeer jullie humor en de gezelligheid die jullie me gedurende de jaren hebben gegeven. Piet en Piet, jullie doorzettingsvermogen kan ik nog een voorbeeld aan nemen. Jullie betrokkenheid heb ik ontzettend op prijs gesteld. Bedankt daarvoor! 
En dan Arend. Lieve Arend, dit jaar zijn we tien jaar samen en naast de fantastische jaren die we in Wageningen en Indonesië hebben gehad, waren de afgelopen jaren in Deventer ook heel bijzonder. Je bent gedurende dit promotietraject mijn grootste maatje geweest; zowel tijdens de dalen als de pieken. Je bent mijn stabiele factor en daarvoor ben ik je enorm dankbaar. Bedankt dat je me weer rustig kon krijgen in tijden van stress. Bedankt dat je me elke ochtend om 7.30 uur uitzwaaide vanuit perron 3 op station Deventer als ik naar Enschede vertrok (en jij met de trein om 7.33 uur naar Utrecht). Je zorgde ervoor dat ik elke dag vrolijk mijn bed uitkwam en uiteindelijk met een glimlach naar werk ging. Ik heb je betrokkenheid enorm gewaardeerd en dit proefschrift is dan ook mede-mogelijk gemaakt door jou. Je bent mijn rots in de branding en ik kan niet wachten om binnenkort met je te trouwen en ons leven voort te zetten in Bennekom! 
About the author 


\section{CARLY JANSSEN (1988)}

Carly was born in Nijmegen, the Netherlands. At the age of eight, her family immigrated to the United States of America where she lived for seven consecutive years. During these years she attended elementary and middle school education. In 2004, at the age of fifteen, her family immigrated back to the Netherlands, and in the following three years, Carly completed her Atheneum at Maaswaal College in Wijchen. She then went to study Nutrition and Health at the Wageningen University and Research, completing both her

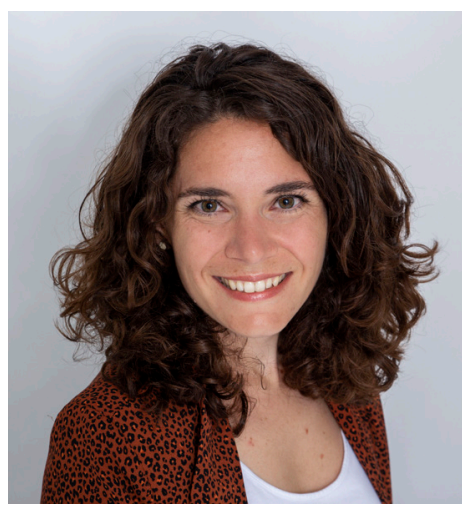
bachelor and master degree in this field, along with a minor immunology. During her studies she primarily specialized in nutritional immunology relating to health and disease outcomes.

After graduating in 2013 and before continuing in her academic career, she worked on various projects for Friesland Campina in Jakarta, Indonesia for almost a year. This experience brought her the inspiration and drive to continue to grow as an academic researcher. At the end of 2014, she started her PhD-research at the University of Twente at the department of Psychology, Health and Technology under supervision of Prof. dr. Mart van de Laar, dr. Martijn Oude Voshaar and dr. Harald Vonkeman, that eventually led to this dissertation.

Her PhD focused on various domains within the field of gout and hyperuricemia, including the clinical management of these diseases, measuring outcomes using patientreported outcome measures and the societal burden of disease. These years have been very meaningful to her as a person, but also as a researcher in gaining comprehensive knowledge within different disciplinary fields. Carly followed and completed various courses on Good Clinical Practice, statistics, pharmacoeconomics, academic research skills and attended various (inter)national conferences in the field of rheumatology, where she presented her research.

In her free time Carly enjoys running, reading, travelling and most of all, spending time with friends and family. 
List of publications 


\section{INTERNATIONAL PUBLICATIONS}

Janssen CA, Oude Voshaar MAH, ten Klooster PM, Vonkeman HE, van de Laar MAFJ. Prognostic factors associated with early gout flare recurrence in patients initiating urate-lowering therapy during an acute gout flare. Clin Rheumatol. 2019; [Epub ahead of print].

Doi: 10.1007/s10067-019-04566-6

Janssen CA, Oude Voshaar MAH, ten Klooster PM, Jansen TLTA, Vonkeman HE, van de Laar MAFJ. A systematic literature review of patient-reported outcome measures used in gout: an evaluation of their content and measurement properties. Health Qual Life Outcomes. 2019;17(1):63.

Doi: $10.1186 / s 12955-019-1125-x$

Janssen CA, Oude Voshaar MAH, ten Klooster PM, Vonkeman HE, van de Laar MAFJ. Development and validation of a patient-reported gout attack intensity score for use in gout clinical studies. Rheumatology. 2019; [Epub ahead of print].

Doi: 10.1093/rheumatology/kez064

Janssen CA, Oude Voshaar MAH, Vonkeman HE, Jansen TLTA, Janssen M, Kok MR, Radovits B, van Durme C, Baan H, van de Laar MAFJ. Anakinra for the treatment of acute gout flares: a randomized, double-blind, placebo-controlled, active-comparator, noninferiority trial. Rheumatology. 2019; [Epub ahead of print].

Doi: 10.1093/rheumatology/key402

Janssen CA, Oude Voshaar MAH, Vonkeman HE, Krol M, van de Laar MAFJ. A retrospective analysis of medication prescription records for determining the levels of compliance and persistence to urate-lowering therapy for the treatment of gout and hyperuricemia in The Netherlands. Clin Rheumatol. 2018;37(8):2291-6.

Doi: 10.1007/s10067-018-4127-x

Janssen CA, Oude Voshaar MAH, Vonkeman HE, Krol M, van de Laar MAFJ. SAT0357 Compliance and persistence to urate lowering therapy for the treatment of gout and hyperuricemia: a retrospective analysis of medication prescription records. Ann Rheum Dis. 2018;77:1044. [abstract]

Doi: 10.1136/annrheumdis-2018-eular.3022 
Janssen CA, Jansen TLTA, Oude Voshaar MAH, Vonkeman HE, van de Laar MAFJ. Quality of care in gout: a clinical audit on treating to the target with urate lowering therapy in real-world gout patients. Rheumatol Int. 2017;37(9):1435-40.

Doi: 10.1007/s00296-017-3777-3

Janssen CA, Jansen TLTA, Oude Voshaar MAH, Vonkeman HE, van de Laar MAFJ. THU0418 A proof-of-concept study: treating to the target with urate lowering therapy in real-world gout patients. Ann Rheum Dis. 2017;76:365-366. [abstract]

Doi: 10.1136/annrheumdis-2017-eular.2377

\section{OTHER (SCIENTIFIC) CONTRIBUTIONS}

Janssen CA, Oude Voshaar MAH, Vonkeman HE, Jansen TLTA, Janssen M, Kok MR, Radovits B, van Durme C, Baan H, van de Laar MAFJ. Anakinra for the treatment of acute gout: results of a multicenter, randomized, double-blind, non-inferiority trial in The Netherlands. Dutch Society for Rheumatology Conference Najaarsdagen, 28 September 2018, Arnhem, The Netherlands. [abstract: oral presentation].

Janssen CA, Oude Voshaar MAH, Vonkeman HE, Jansen TLTA, van de Laar MAFJ. Severe Gout Registry. Dutch Society for Rheumatology Conference Najaarsdagen, 24 September 2015, Arnhem, The Netherlands. [abstract: poster presentation].

Janssen CA, Oude Voshaar MAH. Severe Gout Registry. Rheumatology News International. $2015 ; 6(1): 3$. 
